$$
T-864
$$

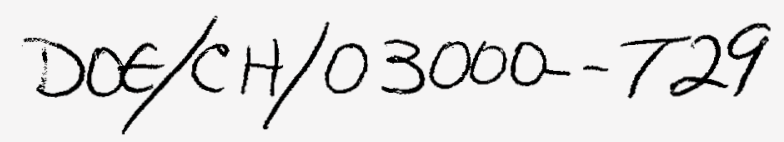

A DISORIENTED CHIRAL CONDENSATE SEARCH AT THE

FERMILAB TEVATRON

by

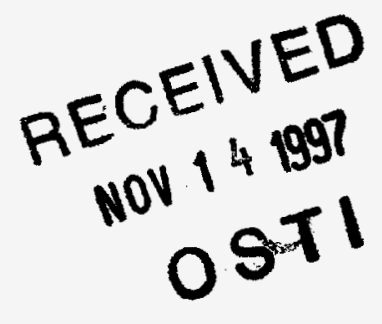

MARY ELIZABETH CONVERY

Submitted in partial fulfillment of the requirements

for the degree of Doctor of Philosophy

Thesis Adviser: Prof. Cyrus Taylor

Department of Physics

CASE WESTERN RESERVE UNIVERSITY

May, 1997

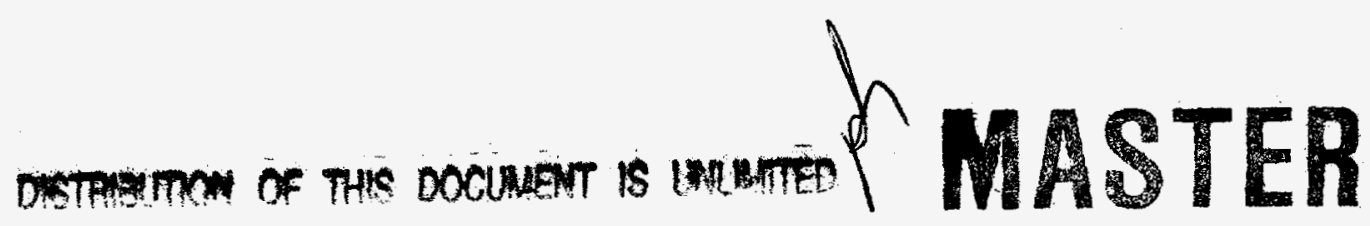




\title{
A DISORIENTED CHIRAL CONDENSATE SEARCH AT THE FERMILAB TEVATRON
}

\author{
Abstract \\ by \\ MARY ELIZABETH CONVERY
}

MiniMax (Fermilab T-864) was a small test/experiment at the Tevatron designed to search for disoriented chiral condensates (DCC) in the forward direction.

Relativistic quantum field theory treats the vacuum as a medium, with bulk properties characterized by long-range order parameters. This has led to suggestions that regions of "disoriented vacuum" might be formed in highenergy collision processes. In particular, the approximate chiral symmetry of $\mathrm{QCD}$ could lead to regions of vacuum which have chiral order parameters disoriented to directions which have non-zero isospin, i.e. disoriented chiral condensates. A signature of DCC is the resulting distribution of the fraction of produced pions which are neutral.

The MiniMax detector at the $\mathrm{C} 0$ collision region of the Tevatron was a telescope of 24 multi-wire proportional chambers (MWPC's) with a lead converter behind the eighth MWPC, allowing the detection of charged particles and photon conversions in an acceptance approximately a circle of radius 0.6 


\section{DISCLAIMER}

This report was prepared as an account of work sponsored by an agency of the United States Government. Neither the United States Government nor any agency thereof, nor any of their employees, make any warranty, express or implied, or assumes any legal liability or responsibility for the accuracy, completeness, or usefulness of any information, apparatus, product, or process disclosed, or represents that its use would not infringe privately owned rights. Reference herein to any specific commercial product, process, or service by trade name, trademark, manufacturer, or otherwise does not necessarily constitute or imply its endorsement, recommendation, or favoring by the United States Government or any agency thereof. The views and opinions of authors expressed herein do not necessarily state or reflect those of the United States Government or any agency thereof. 


\section{DISCLAIMER}

Portions of this document may be illegible electronic image products. Images are produced from the best available original document. 
in pseudorapidity-azimuthal-angle space, centered on pseudorapidity $\eta \approx 4$. An electromagnetic calorimeter was located behind the MWPC telescope, and hadronic calorimeters and scintillator were located in the upstream antiproton direction to tag diffractive events.

The use of standard Monte Carlo simulations for high-energy collisions of elementary particles (PYTHIA) and for interactions of particles in the detector (GEANT) is described, along with the simulation created by the MiniMax Collaboration to generate DCC domains.

A description of the data analysis software is given, including detailed studies of its performance on data from the simulations.

A set of robust observables is derived. These are insensitive to many efficiencies and to the details of the modeling of the parent pion production mechanisms, yet have distinguishable values for DCC and generic chargedneutral distributions. Simulations show that the robust observables are insensitive to detector efficiencies and to systematic errors in the data analysis software.

The resulting values for robust observables for approximately $1.5 \times 10^{6}$ events are shown to be consistent with production by only generic mechanisms. Results from samples of diffractive-tagged events and of high-multiplicity events also show no evidence for DCC. 
To all those who believed in me, especially Jim and Aimeé Convery, Ken Del Signore, Robert Brown, and Cyrus Taylor. 


\section{Acknowledgments}

I owe many thanks, of course, to all my fellow MiniMax collaboration members: T. C. Brooks, W. L. Davis, K. W. Del Signore, T.L. Jenkins, E. Kangas, M. G. Knepley, K.L. Kowalski, C.C. Taylor, S.H. Ob, W.D. Walker,

P. L. Colestock, B. Hanna, M. Martens, J. Streets, R. Ball, H. R. Gustafson, L.W. Jones, M. J. Longo, J.D. Bjorken, A. Abashian, N. Morgan, and C.A. Pruneau. I have learned much from all my collaborators, and have especially benefited from working with $\mathrm{Bj}$ (James Bjorken), Cyrus Taylor, Ken Kowalski, and Tom Jenkins.

Special thanks go to Jon Streets for all his help with computing, to Ken Del Signore for help with the tracker, and to Travis Brooks for many useful conversations.

I am eternally grateful for the opportunity to earn my graduate degree at CWRU which I owe to Robert Brown and Cyrus Taylor.

This work would not have been possible were it not for the support of the Timken Foundation and the CWRU Physics Department. 


\section{Contents}

1 Introduction 1

1.1 Physics motivation $\ldots \ldots \ldots \ldots \ldots \ldots$

1.1.1 Disoriented Chiral Condensates . . . . . . . . . 1

1.1.2 Centauro/anti-Centauro . . . . . . . . . . 4

1.1.3 Multiplicity distributions . . . . . . . . . . 7

1.2 Conceptual design of MiniMax . . . . . . . . . 8

2 MiniMax 15

2.1 The MiniMax environment. . . . . . . . . . 15

2.2 The MiniMax detector . . . . . . . . . . . . . 17

2.2 .1 MWPC telescope . . . . . . . . . . . . 17

2.2 .2 Converter . . . . . . . . . . . . . 21

2.2 .3 Trigger scintillator $\ldots \ldots \ldots \ldots \ldots \ldots . \ldots \ldots 22$

2.2.4 Electromagnetic calorimeter . . . . . . . . . . . 27

2.2 .5 Upstream tags . . . . . . . . . . . . . . . . . 28 
2.3 Run history ....................... 29

$\begin{array}{llr}3 & \text { Simulations } & 49\end{array}$

3.1 PYTHIA ......................... 49

3.2 GEANT ....................... 51

3.2.1 GEANT NHITS study .............. 54

3.2 .2 GEANT trigger rates ............... 58

3.3 DCC generator .................... 59

4 Data Analysis Tools $\quad 81$

4.1 Tracker...................... 81

4.2 Track fitter ................... 84

4.3 Vertexer ...................... 87

4.3.1 Matching tracks at the lead ............ 90

4.3.2 Tracks which may be grouped together . . . . . . . . 92

4.3.3 Tail-less heads as charged tracks . . . . . . . . . . 94

4.3.4 Middle-eight tracks . . . . . . . . . . . . . 95

4.3.5 Origin of fakes .................. 96

5 Generating Function Formalism and Robust Observables 131

5.1 Generating functions for charged-pion-neutral-pion distributions 134

5.2 Generating functions for charged-pion-photon distributions . . 137

5.3 Robust observables . . . . . . . . . . . . . 138 
6.1 DCC: Calculated factorial moments and robust observables . . 147

6.1 .1 Simulations . . . . . . . . . . . . . . 148

6.1.2 Characteristics of events from lead-in runs . . . . . . 152

6.1.3 Characteristics of events with diffractive tags . . . . 153

6.1.4 Characteristics of events with a pbar multiplicity tag . 154

6.2 Multiparticle analysis . . . . . . . . . . . 155

6.3 Low $p_{T}$ photons $\ldots \ldots \ldots \ldots \ldots \ldots \ldots$

7 Conclusions

178

$\begin{array}{ll}\text { A Uncertainty Calculations } & 180\end{array}$

B Track fitting code $\quad 184$

B.1 Track fitter: fit.f. . . . . . . . . . . . . . . 184

B.2 Code for correcting tracks with double hits in the non- $u$ chambers: refit.f . . . . . . . . . . . . . . . 192

$\begin{array}{ll}\text { C Vertexer code } & 200\end{array}$

C.1 Vertexer: vertexer.f . . . . . . . . . . . . . 200

C.2 Code for classifying vertices as charged tracks or photon conversions: chgam.f . . . . . . . . . . . . . . . . . . 212 


\section{List of Figures}

1.1 Binomial and DCC neutral fraction distributions. . . . . . 11

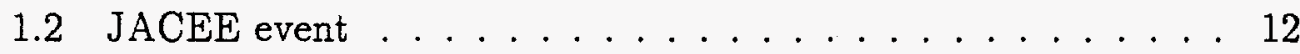

1.3 Charged multiplicity distribution $d N_{c h} / d \eta$ for non-single diffractive inelastic PYTHIA events at $\sqrt{s}=1.8 \mathrm{TeV} \ldots \ldots \ldots 13$

1.4 Original design of the MiniMax detector. . . . . . . . . . 14

2.1 The MiniMax detector before compression of the MWPC telescope. . . . . . . . . . . . . . . . . 31

2.2 New Tevatron beampipe and abort at $\mathrm{C} 0$ and enlarged view of the region from $z \approx 110-190 \mathrm{in} . \ldots \ldots \ldots 32$

2.3 Acceptance in lego space. . . . . . . . . . . . . . 33

2.4 MWPC front view. . . . . . . . . . . . . . . 34

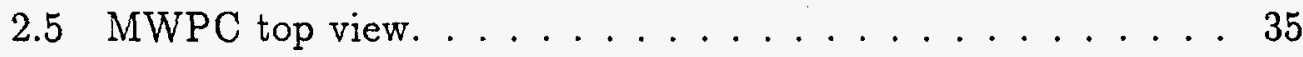

2.6 Pieces of apparatus including pbar and alphabet scintillator, chamber stands, and the original calorimeter. . . . . . . 36

2.7 ADC spectra from some of the trigger counters. . . . . . . . 37 
2.8 TDC spectra from some of the trigger counters. . . . . . 38

2.9 Electromagnetic calorimeter cells in lego space. . . . . . . . . 39

2.10 A view of the detector including the upstream region. . . . . 40

3.1 Pseudorapidity distribution $d N / d \eta$ for charged pions, all charged particles, and photons. . . . . . . . . . . . . 62

3.2 Energy deposited in scintillator and chambers in minimum

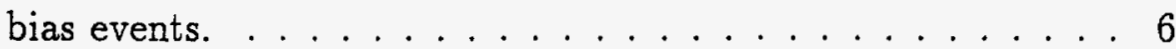

3.3 Energy deposited in scintillator and chambers by single charged

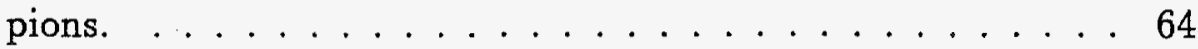

3.4 Mean number of wire hits per chamber: real and GEANT with beam supports, etc. . . . . . . . . . . . . . 65

3.5 Distribution of NHITS: real and GEANT with beam supports,

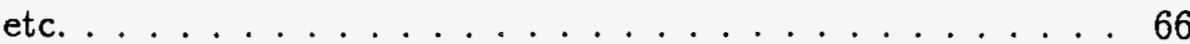

3.6 Pulse height distributions for chambers 1-4, 9-12, real and

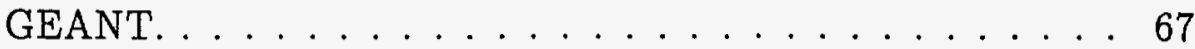

3.7 Pulse height distributions for chambers 21-24: real and GEANT. 68

3.8 Mean number of wire hits in each chamber and with the beampipe removed. . . . . . . . . . . . . . . . . . . 69 69

3.9 Mean number of wire hits in each chamber and with the abort pipe removed. . . . . . . . . . . . . . . 70

3.10 Mean number of wire hits in each chamber and with the chamber frames removed. . . . . . . . . . . . . . 71 
3.11 Mean number of wire hits in each chamber and with the beampipe, abort pipe, and chamber frames removed. . . . . . . . 72

3.12 Charged-particle multiplicity $d N_{c h} / d \eta$ for non-diffractive PYTHIA

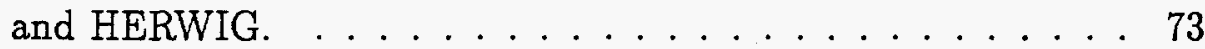

3.13 Momentum (GeV) of DCC pions: $p_{x}$ and $p_{y}$ in lab frame, $p_{z}$ in DCC rest frame, and $p_{z}$ in lab frame. . . . . . . . 74

3.14 Location of the DCC pions in lego space. . . . . . . . . 75

3.15 Distribution of fraction of DCC pions which are neutral $\left(N_{\pi^{0}} / N_{\pi}\right)$ and total number of DCC pions $\left(N_{\pi}\right) \ldots \ldots \ldots 76$

3.16 Number of charged vs number of neutral DCC pions: total number produced, and the number which enter the MiniMax acceptance. . . . . . . . . . . . . 77

3.17 Number distributions of pions produced generically (by PYTHIA). 78

4.1 Event display of a clean charged track. . . . . . . . . 103

4.2 Event display of a straight-through charged track and a charged track which bends in the lead. . . . . . . . . . . . . . 104

4.3 Event display of a photon with three conversion tracks. . . . 105

4.4 Event display of two clean photon conversions. . . . . . . 106

4.5 Display of an event with two charged tracks and a photon, and typical NHITS. . . . . . . . . . . . . . 107

4.6 Display of an event with a large NHITS. . . . . . . . . 108

4.7 Entries in the dst for the events shown in Figs. 4.1 and 4.3. . 109 
4.8 Entry in the dst for the event shown in Fig. 4.5 . . . . . 110

4.9 Histograms of $\chi^{2}$ for the fit in $v$ and of $\left|\theta_{0}-\theta\right| / \sigma_{\theta}$ for re-fitted tails and good tails. . . . . . . . . . . . . 111

4.10 Distance in $u$ and $v$ between photon conversion tracks at the lead when there are two conversion tracks in GEANT. . . . . . 112

4.11 Pointing in $u$ for heads and tails of charged tracks, and for single photon conversion tracks (lead-out run 1093) . . . . . 113

4.12 Pointing in $u$ for heads and tails of charged tracks, and for single photon conversion tracks (lead-in run 1092). . . . . . . 114

4.13 Values of $\log \frac{\chi^{2}}{2}$ for head-tail, head-head, and tail-tail matches at the lead. . . . . . . . . . . . . . 115

4.14 Display of the event shown in Fig. 4.6 for tracks kept by the vertexer. . . . . . . . . . . . . . . 116

4.15 Efficiencies for finding charged tracks as a function of energy and transverse momentum. . . . . . . . . . . . . 117

4.16 Efficiency for finding photons as a function of photon energy. . 118

4.17 Efficiency for finding photons as a function of photon transverse momentum. . . . . . . . . . . . . . . . . . . 119

4.18 Efficiency for finding charged tracks and photons as a function of total multiplicity into the acceptance. . . . . . . 120 
4.19 Efficiency for finding charged tracks as a function of charged multiplicity into the acceptance, efficiency for finding photons vs photon multiplicity. . . . . . . . . . . . . . 121

4.20 Mean number of fake charged tracks and photons found per event with a given multiplicity. . . . . . . . . . 122

4.21 Efficiency for finding charged tracks and photons as a function of NHITS of the event. . . . . . . . . . . . 123

4.22 Mean number of fake charged tracks and photons found per event with a given NHITS. . . . . . . . . . . . . 124

4.23 Efficiencies for finding charged tracks, photons at a given $\eta . \quad 125$

4.24 Efficiencies for finding charged tracks, photons at a given $\phi . \quad 126$

4.25 Location of tracks at the lead in phase space $\left(u, \theta_{u}\right) \ldots . . .127$

4.26 PYTHIA charged-track and photon multiplicity distributions, mean multiplicities vs NHITS for all lead-in GEANT events and for non-vetoed events. . . . . . . . . . . 128

4.27 Observed photon multiplicity distribution for all events and for non-vetoed events. . . . . . . . . . . . . . . 129

5.1 Contour plots of the generating function $G\left(z_{c h}, z_{0}\right)$ and $\log G\left(z_{c h}, z_{0}\right)$ from all PYTHIA charged and neutral pions, and from those entering the MiniMax acceptance. . . . . . . . . . . 145 
5.2 Contour plots of the generating function $G\left(z_{c h}, z_{0}\right)$ and $\log G\left(z_{c h}, z_{0}\right)$ from all charged and neutral pions from the DCC generator, and from those entering the MiniMax acceptance. . . . . . 146

6.1 Histogram of $(r(i)-\langle r\rangle) / \sigma_{r}(i)$ for groups (i) of 25000 events and Gaussian fit. . . . . . . . . . . . . 161

6.2 ADC values of the hadronic calorimeters which see anti-neutrons

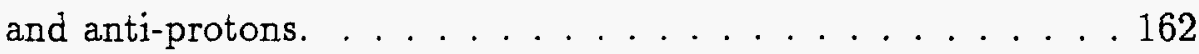

6.3 Energy (in $\mathrm{MeV}$ ) deposited in the pbar counters vs number of charged particles hitting these counters . . . . . . . 163

6.4 PED_SUB_ADC values (in units of mip energies or, equivalently, number of charged particles) of the pbar counters . . . 164

6.5 Pseudorapidity distribution $d N / d \eta$ vs $\eta$ for charged tracks in the data, GEANT, and PYTHIA, uncorrected for efficiencies. 165

6.6 Pseudorapidity distribution $d N / d \eta$ vs $\eta$ for photons in the data, GEANT, and PYTHIA, uncorrected for efficiencies. . . . 166

6.7 Histogram of the $z$ of closest approach between all heads, and of the $\chi^{2}$ of the matching of those pairs in GEANT. . . . . . 167

6.8 Histogram of the $z$ of closest approach between all heads, and of the $\chi^{2}$ of the matching of those pairs in the data. . . . 168

6.9 Opening angle between pairs found as window conversion tracks in GEANT, $p_{T}$ of all primary photons in GEANT, and efficiencies for finding window conversions as a function of $p_{T} \ldots \ldots 169$ 
6.10 Contour plots of the $p_{T}$ found by bending in the scintillator and by using the calorimeter vs the actual $p_{T}$ of the converted photon in GEANT, and corresponding $p_{T}$ distributions. . . . 170

6.11 Distributions of $p_{T}$ found by bending in the scintillator and by using the calorimeter for converted photons in the data. . . 171 


\section{List of Tables}

2.1 Old Tevatron beampipe and abort at $\mathrm{C} 0 \ldots \ldots \ldots$. . . . . 41

2.2 New Tevatron beampipe and abort at C0. . . . . . . . . 42

2.3 Chamber alignment from $2 / 16 / 94 \ldots \ldots \ldots \ldots$

2.4 Chamber alignment from $11 / 23 / 94 \ldots \ldots \ldots 4$

2.5 Chamber alignment with 11 chambers parallel from $3 / 19 / 95$. . 45

2.6 Chamber alignment after compression from $3 / 24 / 96$. . . . . 46

2.7 Configuration of alphabet counters for runs with the old beampipe, through 1994. . . . . . . . . . . . . . . . 47

2.8 Configuration of alphabet counters during running with the new beampipe from $2 / 95-7 / 95 . \ldots \ldots \ldots 4$

2.9 Configuration of alphabet counters during runs with compressed MWPC telescope. . . . . . . . . . . . . . 47

2.10 Running conditions from some January 1996 runs. . . . . . . 48

3.1 PYTHIA cross sections and GEANT trigger rates $\ldots \ldots .79$ 
3.2 NHITS of "Michgan chambers" in GEANT (threshold $=20$ ) for varying pulse-height and scintillator cuts. . . . . . . 80

4.1 Cuts used by the tracker. . . . . . . . . . 130

6.1 Robust observables $r_{i, j}$ for generic events simulated by PYTHIA and pure-DCC events simulated by the DCC generator, along with predictions for binomial and $1 /(2 \sqrt{f})$ distributions. . . . 172

6.2 Results for varying parameters in the DCC generator. . . . . 173

6.3 The effect on the $r_{i, 1}$ of an admixture of DCC and generic (PYTHIA) events. . . . . . . . . . . . . . 174

6.4 Number of events with a given $n_{c h}, n_{\gamma} \ldots \ldots \ldots 175$

6.5 Values of $r_{i, j}$ for all lead-in events and for those with diffractive

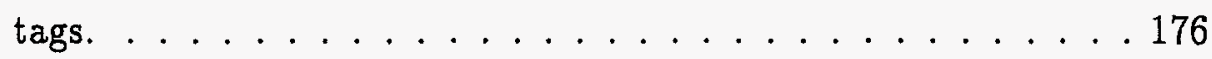

6.6 Mean multiplicities and robust observables for bins of pbar multiplicities each containing $10 \%$ of the events. . . . . . 177 


\section{Chapter 1}

\section{Introduction}

\subsection{Physics motivation}

\subsubsection{Disoriented Chiral Condensates}

The Lagrangian of QCD (Quantum Chromodynamics [1], the theory of strong interactions) for two quarks ( $u$ and d) has isospin symmetry $S U(2)$. In the limit that the quarks are massless, the Lagrangian also has chiral symmetry $\mathrm{SU}(2)_{L} \times \mathrm{SU}(2)_{R}$. That is, if we write the isospin doublet $\Psi=\left(\begin{array}{l}u \\ d\end{array}\right)$ in terms of left- and right-handed fields,

$$
\Psi=\frac{1}{2}\left(1-\gamma_{5}\right) \Psi+\frac{1}{2}\left(1+\gamma_{5}\right) \Psi=\Psi_{L}+\Psi_{R}
$$


where $\gamma_{5}$ is the usual product of gamma matrices of Dirac theory, then the Lagrangian is symmetric under $\Psi_{L} \leftrightarrow \Psi_{R}$. The spontaneous breaking of this chiral symmetry in the QCD ground state is accompanied by a massless Goldstone boson [2], the pion. In the real world, the quarks are light but not massless, and the QCD Lagrangian has an approximate chiral symmetry which is explicitly broken, giving the pion a small mass.

Spontaneous chiral symmetry breaking is often described by the linear sigma model [3], in which there is a scalar field $\sigma$ of isospin 0 and a vector pion field $\vec{\pi}$ of total isospin 1 . The Lagrangian can be written as

$$
\mathcal{L}=\frac{1}{2}\left(\partial_{\mu} \vec{\pi} \partial^{\mu} \vec{\pi}+\partial_{\mu} \sigma \partial^{\mu} \sigma\right)-\frac{\lambda}{2}\left(\vec{\pi}^{2}+\sigma^{2}-f_{\pi}^{2}\right)^{2}
$$

and the potential is minimized for $\sigma^{2}+\vec{\pi}^{2}=f_{\pi}^{2}$. The symmetry is broken when a particular minimum is chosen. In order to break chiral symmetry, but not isospin symmetry, the minimum chosen is that for which the $\sigma$ field acquires a vacuum expectation value: $\langle\sigma\rangle=f_{\pi},\langle\vec{\pi}\rangle=0$. A term can be added to the Lagrangian in order to break the chiral symmetry explicitly, such as $f_{\pi} m_{\pi}^{2} \sigma$ or $-\frac{1}{2} m_{\pi}^{2} \vec{\pi}^{2}$, but it is unclear experimentally which of these terms is realized in nature [4].

The ordinary vacuum has chiral order parameter in the $\sigma$ direction (it has no isospin). A disoriented chiral condensate (DCC) is a piece of vacuum which is disoriented from the $\sigma$ direction to a direction with $\vec{\pi}$ components. When the DCC domain makes contact with the ordinary vacuum, it coher- 
ently radiates pions with isospin determined by the direction of disorientation in order to restore the $\sigma$ direction. For example, if the disorientation were in the $\pi^{0}$ direction, $\pi^{0}$ 's would be emitted. There has been much theoretical work done recently on DCC and other mechanisms for coherent semiclassical radiation of pions in high-energy collisions of hadrons and of heavy ions [5]-[16].

The distinctive signature of DCC is that the pions produced when the DCC domain makes contact with the outside vacuum have a neutral fraction $f=N_{\pi^{0}} /\left(N_{\pi^{+}}+N_{\pi^{-}}+N_{\pi^{0}}\right)$ distributed according to

$$
P(f) d f=\frac{1}{(2 \sqrt{f})} d f
$$

in the limit of large numbers of pions. This neutral fraction distribution is common to some other mechanisms which produce coherent final states [13]-[14], [17]-[19]. The proof of this has been given in terms of quantum mechanical coherent state arguments [10], but can be seen easily with a geometrical argument. The vacuum condensate has equal probability of having an order parameter oriented in any direction in $(\sigma, \vec{\pi})$ space. If we define $\theta$ as the polar angle relative to the $\pi^{0}$-axis, quantum-mechanical arguments give $f=\cos ^{2} \theta$, and $P(f) d f=P\left(\cos ^{2} \theta\right) d(\cos \theta)$. We have $d f / d(\cos \theta)=2 \sqrt{f}$, so that $P(f) d f=(1 / 2 \sqrt{f}) d f$. In generic particle production, that is, production by (observed) mechanisms other than DCC, producing a pion of any given charge is equally likely due to isospin symmetry, so that $f$ is binomially 
distributed with mean $\langle f\rangle=\frac{1}{3}$. Note that the $1 /(2 \sqrt{f})$ distribution also has a mean of $\langle f\rangle=\frac{1}{3}$, but that the distributions are quite different; in particular, the probability of producing pions with a small or large neutral fraction is much higher for DCC than for generic production (Fig. 1.1).

It is conjectured that such a condensate may be formed in hadron-hadron collisions with high transverse energy and a large multiplicity (number of particles produced). As the collision debris expands outward at almost the speed of light, it may form a hot, thin shell, the cool interior of which would be separated from the outside vacuum and could conceivably have a chiral order parameter which is disoriented from the $\sigma$ direction. When the shell hadronizes, the interior condensate makes contact with the outside vacuum and radiates pions.

\subsubsection{Centauro/anti-Centauro}

Cosmic ray experiments have found evidence for hadronic events which can be interpreted as having an anomalously large or small fraction of pions which are neutral and may therefore be related to DCC. Centauro events have been observed in emulsion chambers by the Chacaltaya-Pamir Collaboration $[20,21]$ and are characterized by a large number $(\sim 100)$ of charged particles and almost no electromagnetic energy, which implies no $\pi^{0}$ s, since their immediate decay produces two photons. The center-of-mass $(\mathrm{cm})$ energies are on the order of a $\mathrm{TeV}$ or larger. Further interpretation of the events is 
controversial. What is measured can be related to the transverse momentum, e.g., $k_{\gamma}\left\langle p_{T}\right\rangle=0.35 \pm 0.15 \mathrm{GeV}$ for Centauro I, but the gamma ray inelasticity $k_{\gamma}$ is not known. The value is usually quoted as $k_{\gamma} \sim 0.2-0.4$ with the lower and upper values preferred for nucleons and pions, respectively. Most analyses assume that the hadrons produced in Centauros events are nucleons. However, if we want to interpret Centauros as being related to DCC, the hadrons should be pions, and therefore it is possible that the $p_{T}$ is low $\left\langle\left\langle p_{T}\right\rangle \approx\right.$ $0.875 \pm 0.375 \mathrm{GeV}$ with a large systematic uncertainty), which is referred to as Chiron behavior. It has been suggested (Ref. [22] based on data from Ref. [21]) that these events may be diffractive. ${ }^{1}$ Taking the view that Centauros are diffractive fireballs recoiling against a proton or anti-proton, but that pions rather than nucleons are produced, boosting these events to the lab frame of Fermilab Tevatron collisions, Centauros would be expected to occur at $\eta \sim 3.5-4.5 .^{2}$ (As will be discussed in Sec. 2.2, the acceptance of the MiniMax detector covers precisely this region.)

\footnotetext{
${ }^{1}$ Diffractive processes [23] are thought of as involving the exchange of a colorless object called a pomeron, but are not well understood. In single diffraction, either the incoming proton or anti-proton is dissociated, while the other remains intact and typically has a low transverse momentum and a longitudinal momentum almost that of the initial beam. Both hadrons are dissociated in double diffraction.

${ }^{2}$ We work in what is called "lego space" where $\phi$ is the azimuthal angle and $\eta$ is the pseudorapidity which is defined in terms of $\theta$, the angle from the beam axis, as $\eta=$ $-\ln \tan \frac{\theta}{2}$. Therefore, the region explored by central detectors which look transverse to the beam covers small $\eta$, while $\eta$ gets infinitely large in the beam direction. An advantage to using the pseudorapidity is that $\eta$ has a simple transformation under boosts in the beam direction. Also, the density of particles produced in a collision is uniform over a large region of lego space.
} 
Events with a large neutral fraction are referred to as anti-Centauros. Such cosmic ray events have been reported by the JACEE Collaboration [24], which uses balloon-borne detectors. An example is the event shown in Fig. 1.2. The leading cluster contains about 32 photons and only 1 charged particle. A possibly distinct cluster has about 54 photons and 17 charged particles. The collision occurred within the detector with a cm energy greater than $200 \mathrm{GeV}$ and Chiron behavior was exhibited.

However, the interpretation of these cosmic ray events is controversial, and observations of this type of event under controlled conditions, such as in a collider environment, where they could be better understood, would be very useful. Several collider experiments have unsuccessfully looked for large Centauro-like domains. CDF has conducted such a search at the Tevatron $(\sqrt{s}=1.8 \mathrm{TeV})$, and has reported no evidence for Centauros or antiCentauros [25]. However, the CDF search only covered the region $|\eta| \lesssim 3$ and only looked at particles with $p_{T} \gtrsim 400 \mathrm{MeV}$, and therefore might not be expected to observe such phenomena. The UA5 Collaboration ruled out large Centauro domains going out to larger $\eta[26,27]$, but at $\mathrm{cm}$ energies no greater than $900 \mathrm{GeV}$, whereas the necessary $\mathrm{cm}$ energy is expected to be larger. UA1 also conducted a search at relatively low energies and in the central region, and found no evidence [28]. 


\subsubsection{Multiplicity distributions}

Very little is known about particle production in the forward direction because this region has not been well studied. The distribution of charged particles in lego space has been reported by experiments such as CDF [29] and UA1 [30] for the central region $(|\eta| \lesssim 3)$. Measurements of $d N_{c h} / d \eta$ were made for larger $\eta$ by UA5 [27], and by P238 [31] at the CERN Sp̄̄S collider for proton-anti-proton collisions at center-of-mass energy $\sqrt{s}=630 \mathrm{GeV}$. The distribution of photons $d N_{\gamma} / d \eta$ in the forward direction is even less well known; UA5 observed photons at $\sqrt{s} \leq 900 \mathrm{GeV}$ [27, 32]. MiniMax, which was able to observe both charged particles and photons in the region $3.3<\eta<4.5$ at $\sqrt{s}=1.8 \mathrm{TeV}$, therefore had the potential to make measurements in a previously unexplored region.

Figure 1.3 shows a plot of $d N_{c h} / d \eta$, averaged over typical collisions at Tevatron energies, from the event generator PYTHIA. The distribution in the central region is taken from measured values, and these are extrapolated to the forward region. Note that the mean number of charged particles varies by only about 0.5 (about $13 \%$ ) in the region $|\eta|<4$. Since there is no preferred azimuthal direction, the distribution in $\phi$ is also uniform. 


\subsection{Conceptual design of MiniMax}

With the primary goal to search for DCC, the MiniMax detector should be able to observe both charged particles and photons simultaneously. If we take seriously the interpretation of Centauros and anti-Centauros given in Sec. 1.1.2, the detector should cover the forward region $3.5<\eta<4.5$, and be sensitive to low- $p_{T}$ particles. The smallest coverage which would be expected to be sufficient for observing such events is $\Delta \eta \Delta \phi=1$. Further considerations include the restricted area around the beampipe due to the main ring and the floor, and the lack of funding for the experiment. In order to achieve "minimal maximum acceptance" (hence the name MiniMax), the detector was designed as a telescope of multi-wire proportional chambers (MWPC's) along the beampipe, with converter inside the telescope. Thus, charged particles can be observed in the chambers before and after the converter, and photon conversions in the chambers behind the converter.

In the absence of a magnetic field, the energy of the particles can only be determined using calorimetry. For this reason, and to observe photons which do not convert inside the telescope, an electromagnetic calorimeter was placed behind the MWPC telescope.

If Centauros are diffractive and are related to DCC, then DCC might be more likely to be produced in diffractive interactions than in non-diffractive ones. In order to test this conjecture, diffractive events must be identified. Scintillator and hadronic calorimetry were used to detect leading anti-protons 
and anti-neutrons from diffractive events.

A picture of the original design for the MiniMax detector is shown in Fig. 1.4 .

A brief note on the similarity between MiniMax and UA5 is in order, although the details of the UA5 experiment did not play a role in the design of the MiniMax detector. UA5 was an experiment at the CERN protonanti-proton collider ( $\mathrm{Sp} \overline{\mathrm{p} S})$ and ran from 1981-1982 and in 1985, during which time collisions at $\sqrt{s}=546,200$, and $900 \mathrm{GeV}$ were studied. The detector consisted of two streamer chambers, $600 \times 125 \times 50 \mathrm{~cm}^{3}$, on opposite sides of the beampipe, in which charged particles left tracks which were photographed for analysis by well developed techniques. For the runs at $\sqrt{s}=546 \mathrm{GeV}$, photons were detected through conversions in lead-glass plates, approximately $1 \mathrm{X}_{0}$ thick, located inside the streamer chambers near the sides. In later runs, a lead converter plate $(2 \mathrm{~mm}$ of lead supported by an aluminum box with walls $1 \mathrm{~mm}$ thick), placed between the beam pipe and the upper streamer chamber, was used instead. The beampipe was elliptical with mean dimensions $6 \times 15.2 \mathrm{~cm}^{2}$. Combining this transverse distance of the chambers from the beam axis with the length of the chambers, UA5 was able to observe particles at pseudorapidities up to $|\eta| \approx 5$. UA5 published results on many important studies, including multiplicity and pseudorapidity distributions and correlations for charged particles and photons, strangeness production (e.g. K's, $\Lambda$ 's, and $\Xi$ 's, observed through decays), diffractive 
dissociation, and a search for Centauros. Reference [27] is a comprehensive report on these studies at $\sqrt{s}=546 \mathrm{GeV}$. The Monte Carlo simulations created and used by UA5, which include non-diffractive and diffractive event generators, along with a Centauro generator, are described in Ref. [33]. 


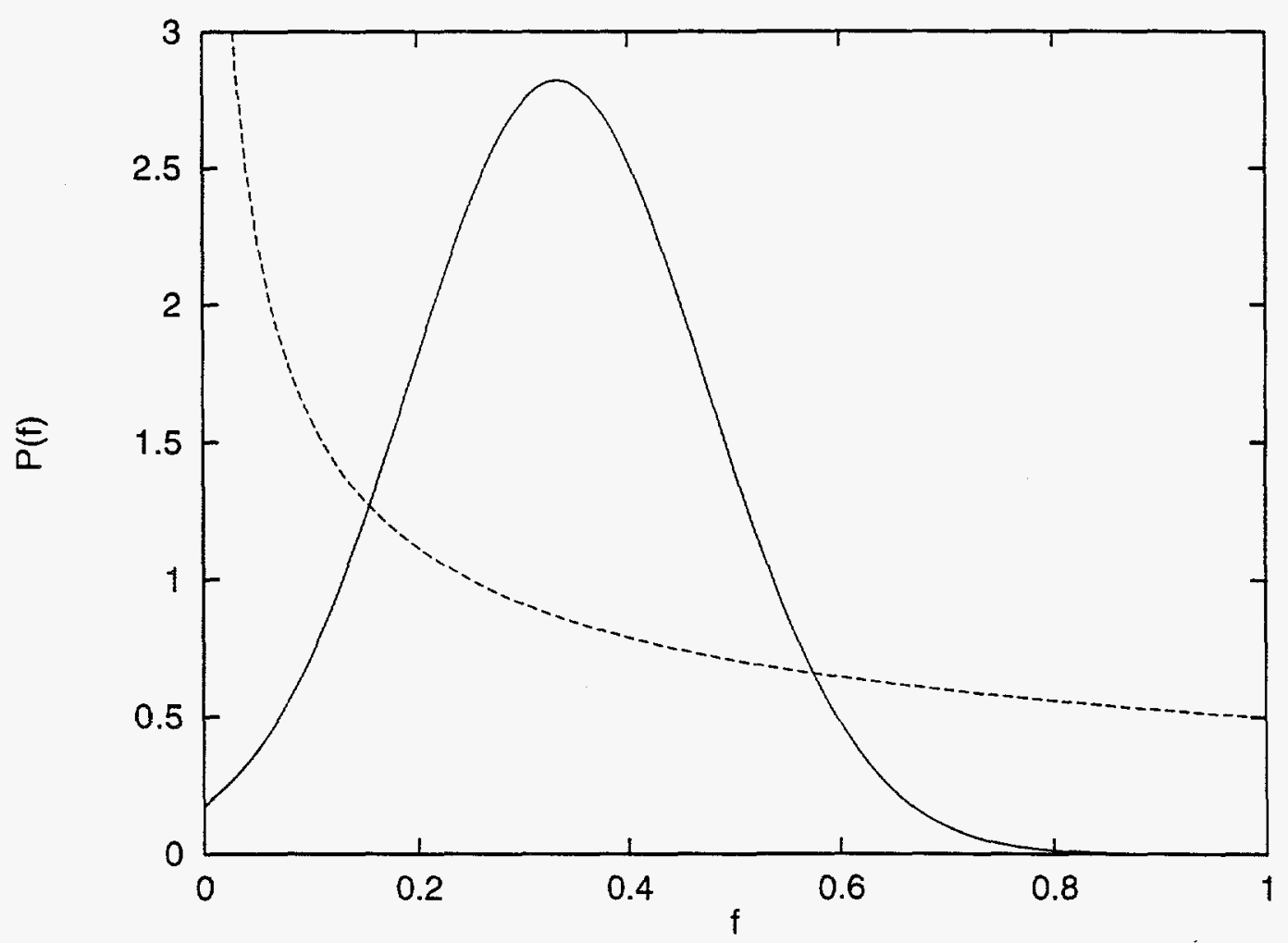

Figure 1.1: Binomial and DCC neutral fraction distributions. 
Figure 1.2: JACEE event 


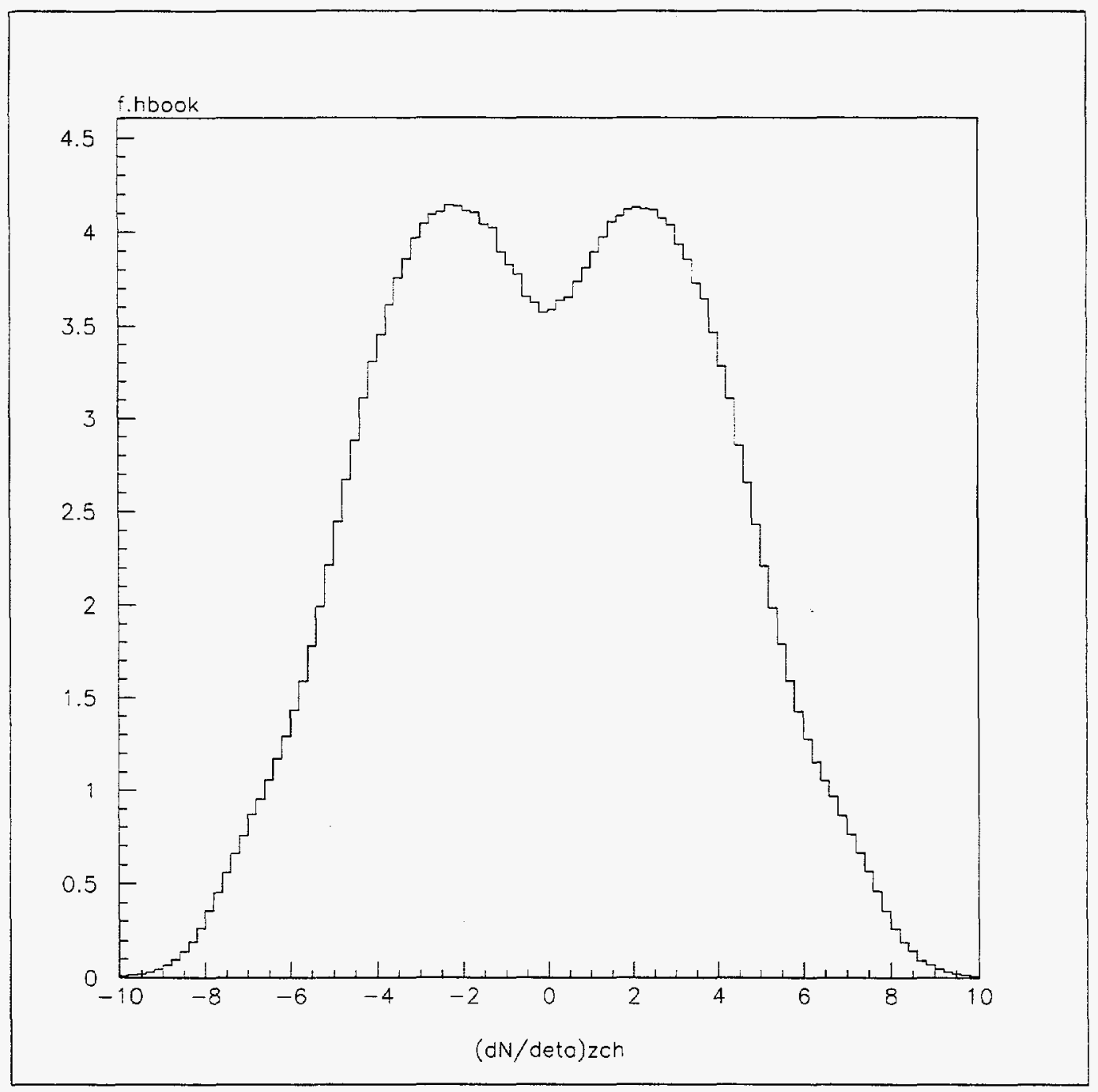

Figure 1.3: Charged multiplicity distribution $d N_{c h} / d \eta$ for non-single diffractive inelastic PYTHIA events at $\sqrt{s}=1.8 \mathrm{TeV}$. 


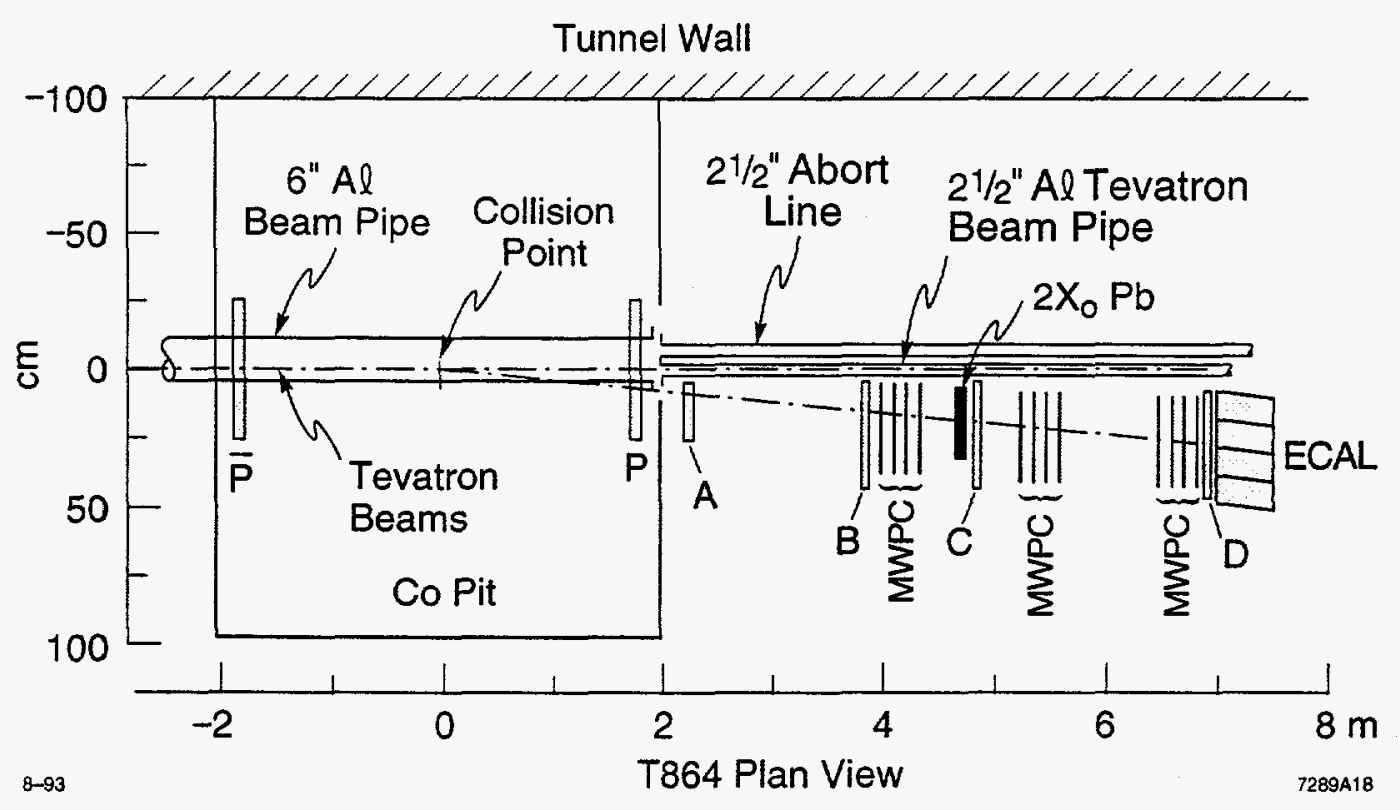

Figure 1.4: Original design of the MiniMax detector. 


\section{Chapter 2}

\section{MiniMax}

\subsection{The MiniMax environment}

The MiniMax detector was located at the $\mathrm{C} 0$ collision region of the Fermilab Tevatron, which collides protons and anti-protons at $\mathrm{cm}$ energy $\sqrt{s}=1.8$ $\mathrm{TeV}$. A sketch of the detector is shown in Fig. 2.1. The coordinate system was taken to be left-handed with positive $z$ in the downstream proton direction and positive $y$ upwards. The region included a pit which was 18 in deep, 60 in wide, and 170 in long, with the bottom centered at $(x, y, z)=(0,-29$ in, 1 in $)$ relative to the nominal collision point defined as $(0,0,0)$. (Note that the actual collision point as determined by the mean distance of closest approach between tracks is found to be at $z \approx 7$ in, and within 0.5 in of $x=y=0$. The pointing of the tracks will be discussed in more detail in Sec. 4.3.)

The Tevatron beampipe in the neighborhood of the $\mathrm{C} 0$ collision point 
during early stages of the experiment (from late 1993 to early 1995) was a 6indiameter, 0.0625 in-thick $\mathrm{Al}$ pipe, with a 2 in-thick $\mathrm{Al}$ flange at the transition to a 2.5 in-diameter, 0.035 in-thick $\mathrm{Al}$ pipe and an abort pipe of the same dimensions. The detailed dimensions and locations of the pipe segments are given in Table 2.1. Secondaries from interactions of collision primaries in the flange dominated the particles observed in the forward region.

A new beampipe was designed to minimize the number of interactions in the pipe which produced background hits in the detector. This was accomplished by steps of increasing diameter, with flared transitions rather than vertical plates wherever possible between two sections of pipe (the pipe bent out at a $30^{\circ}$ angle to meet the adjoining segment), and by a thin window at $z=120$ in through which all collision primaries entering the detector would pass. The steps in the Al pipe are given in Table 2.2, and the beampipe and abort are pictured in Fig. 2.2. The new pipe was installed in February 1995, and was in place for the majority of the data collected.

The main ring, which was used for accelerating protons which were then used in production of the anti-protons, and a main ring abort pipe were present in the $\mathrm{C} 0$ collision region, both approximately 20 in above the Tevatron pipe. These pipes were steel, on the order of $1 / 4$ in thick. The main ring and abort were about 6 in and 4 in in diameter, respectively. The accelerating protons in the main ring interacted with particles present in gas inside the pipe, producing blinding flashes of background in the detector, so that 
the triggers had to be gated off when the main ring protons passed through the collision region. In events which were triggered on, such beam-gas interactions also created hits in the upstream scintillator used for diffractive tags (which will be discussed in Sec. 2.2). Interactions of collision primaries with the main ring pipes produced background tracks in the detector. And accelerator studies using the main ring produced large bursts of radiation which badly damaged some of the electronics. This was the greatest source of radiation damage because the spread in momentum of beam particles in the (older) main ring was much greater than that for the Tevatron beams, and those particles with less-than-beam momentum often interacted in the pipe or escaped the pipe and interacted elsewhere.

\subsection{The MiniMax detector}

\subsubsection{MWPC telescope}

Particles were tracked through a telescope of 24 multi-wire proportional chambers on the downstream proton side of the collision point. The acceptance of these chambers was approximately a circle of radius 0.6 units in $\eta-\phi$ space centered on $\eta \approx 4$ (see Fig. 2.3). The chambers were designed for past experiments at CWRU [34]. Figures 2.4 and 2.5 show diagrams of the wire chamber design. Each chamber had an active volume of 12.8 in $\times 12.8$ in $\times 0.375$ in containing 128 parallel wires with a spacing of 
0.1 in. The anode wires were gold-plated tungsten with a 0.0008 in diameter, and the cathode plane consisted of an evaporated Al film on one side of 0.001 in-thick mylar. The gap was $3 / 16$ in. Copper clad surfaces $1 / 16$ in from the signal plane served as guard rings, and $3 / 16$ in from the signal plane as the contact for the high-voltage plane. The $80 \% \mathrm{Ar}, 20 \% \mathrm{CO}_{2}$ gas used was circulated freely on both sides of the aluminized mylar. The outer faces were sealed with 0.005 in-thick mylar. After tests in early 1995 determined that some of the chambers were sensitive to light, 0.002 in-thick black plastic film (kevlar, or similar material) was added to the front and back faces of the chambers.

The chambers were held together by $1 / 8 \mathrm{in}$ - and $1 / 16$ in-thick frames made of G-10 which were epoxied together. Grooves 0.004 in-wide were etched in the printed circuit board face of the signal plane to keep the signal wires aligned during the epoxy process. The $1 / 16$ in-thick G-10 circuit board extended 1.85 in below the frame, which was 1 in in width, so that the completed chambers had a width of $14.8 \mathrm{in}$, and a height of $16.65 \mathrm{in}$.

Two types of readout electronics were used. Half of the chambers used nanometrics supplied by Fermilab, which did not give pulse-height information, but were very reliable. The other half were equipped with cards made by the University of Michigan. These had 12 bits to digitize the signal from each wire, and gave pulse-height information about the amount of charge deposited on the wire. A certain chip in the amplifier cards was had to be 
replaced frequently due to radiation damage, which often led to runs which did not have all MWPC's functioning. ${ }^{1}$

The chambers were held in position by aluminum stands which allowed them to be rotated at various angles. The original stands each held four chambers. New stands were made in September of 1995 to hold eight chambers in order to compress them closer together. An end-on view of a chamber stand is pictured in Fig. 2.6, along with other pieces of apparatus.

Chamber orientations for various periods of running are given in Tables 2.3-2.6. The alignments during the first year or so of running utilized largeangle stereo for three-dimensional resolution. Each chamber was rotated by a different angle in order to reduce the number of potential reconstructed tracks as described below. This philosophy was reconsidered [35], and for reasons discussed below, the chambers were reconfigured in February 1995.

The final orientation of the chambers was defined in a coordinate system where, looking in the positive $z$ direction, the $(u, v)$-axes were a $45^{\circ}$ counterclockwise rotation of the $(x, y)$-axes about the $z$-axis. [We also redefine the azimuthal angle as $\phi=\tan ^{-1}(v / u)$.] In the chamber configuration used for the majority of the data taking, three of the front chambers and eight of the rear had their wires aligned perpendicular to the $u$-axis (" $u$ chambers"), three of the front chambers had wires aligned within $15^{\circ}$ of normal to the $v$ -

\footnotetext{
${ }^{1}$ For a brief period in Dec. 1995, the Tevatron ran at $\sqrt{s}=630 \mathrm{GeV}$. However, all of the data collected by MiniMax at that energy did not have information from the wire chambers which were read out using the Michigan electronics because these were under repair at the time.
} 
axis (" $v$ chambers"), and the remainder were rotated by small angles $\left(4-15^{\circ}\right)$ from the $u$-chamber orientation (" $u$ ' chambers").

A configuration with wires in half the chambers aligned in a given direction and the other half perpendicular gives equal resolution in both directions. However, the number of potential tracks considered by a track-finding algorithm goes like the square of the number of real tracks. For example, two real tracks will produce two hits in a front chamber and two in a back chamber, leading to four potential track candidates if such candidates are found using only the two chambers. In a situation like that of MiniMax, where there are many random hits due to pipe shower, confirming hits in other chambers can make these extra track candidates look like actual tracks. The opposite extreme is a chamber configuration with all wires parallel. This does not give any information about the location of a track in the direction along the wires, but greatly reduces the number of potential fake tracks. The other great advantage to parallel wires, as opposed to large-angle stereo at random angles, is that tracks can be reconstructed by eye in a two-dimensional display of the hit wires, which can be quite useful for studying the properties of, e.g., tracks coming from interactions in the beampipe.

The MiniMax Collaboration determined that the configuration discussed above, employing small-angle stereo, was a good compromise between the two extremes because it allowed for some resolution in both directions, the ability to view wire hits in about half the chambers, and reduced probability 
of finding fake tracks. Note also that the front chambers had three $v$ chambers for increased resolution of charged tracks ${ }^{2}$ and of their intersection with the $z$-axis (i.e. the collision point); the number of wire hits, especially of those due to pipe shower, was much smaller in the front chambers than in the back, so that the reduction of fakes by small-angle stereo would not have been as much of an advantage as it was in the rear chambers.

Another major reconfiguration was performed in the fall of 1995 in which the chambers were moved closer to the collision point. The reason was that pipe shower and other sources of background increased towards the rear of the detector. This will be discussed further in Sec. 3.2.1.

\subsubsection{Converter}

In order to detect photons, converter was inserted behind the eighth chamber. During early running (through 1994), the converter was a stationary plane of lead, 6 in $\times 4$ in $\times 1 \mathrm{X}_{0},{ }^{3}$ centered on (5.25in, $\left.6.20 \mathrm{in}, 198 \mathrm{in}\right)$, level with the floor. For runs after Feb. 1995, the movable converter was an 8 in $\times 8$ in square, rotated by $45^{\circ}$ relative to the floor, so that its edges were parallel to the $(u, v)$ axes. Various thicknesses and materials were used to study systematics in

\footnotetext{
${ }^{2} \mathrm{~A}$ charged track is defined as the reconstructed track from a charged particle which appears to originate in the collision.

${ }^{3}$ One radiation length $\left(1 \mathrm{X}_{0}\right)$ is defined as the mean distance over which a high-energy electron loses all but $1 / e$ of its energy by bremsstrahlung, and varies with atomic number and mass of a medium. Also, for very high-energy photons, the $\mathrm{e}^{+} \mathrm{e}^{-}$pair-production cross section is given by $\sigma \approx \frac{7}{9}\left(A / \mathrm{X}_{0} N_{A}\right)$, where $A$ is the atomic mass and $N_{A}$ is Avagadro's number [36].
} 
detecting photon conversions; these were: $1,2,0.5 \mathrm{X}_{0}$ lead, $1 \mathrm{X}_{0}$ iron, and no converter (converter in the "out" position). For runs before chamber compression, the lead was located at ( 7.7 in, 0.1 in, 184.8 in) for "lead-in" running, and approximately 20 in farther from the beampipe in $x$ for "leadout" runs. For the production runs, $1 \mathrm{X}_{0}$ lead was used about equally often in the lead-in position ( $5.13 \mathrm{in}, 5.13 \mathrm{in}, 150 \mathrm{in}$ ), and in the lead-out position.

\subsubsection{Trigger scintillator}

Scintillator counters were used to trigger on particles passing through the detector, signaling a collision. The counters had both ADC (analog to digital converter) readout, which gave information about the energy deposited by particles passing through the scintillator, and TDC (time to digital converter) information about the time-of flight of the particles. The signature of beam-beam (rather than beam-gas) collisions is correctly-timed hits in the scintillator arrays.

The scintillator counters which were interspersed among the chambers were referred to as A-E, or collectively as "alphabet counters". The B and D counters which played a role in the usual trigger were located one directly behind the lead and one directly behind the last chamber. Each was comprised of two 8 in $\times 16$ in $\times 0.5$ in pieces which together formed a square, which was rotated into the $(u, v)$ frame in a similar manner as the lead. The $\mathrm{C}$ counter was of the same type as B and D. In early runs, B and C were located on ei- 
ther side of the converter, and signals from both were required for the trigger, significantly reducing the probability of triggering on an event with a photon conversion but no charged tracks. The smaller A counter, located in front of the MWPC telescope, and the larger E counter behind the calorimeter were also sometimes used in the trigger. The history of configurations is given in Tables 2.7-2.9.

The trigger also required a hit in scintillator counters on the downstream anti-proton side of the collision ("pbar counters"). This array contained four 12.5 in $\times 2$ in $\times 1$ in pieces and four 12.5 in $\times 4$ in $\times 1$ in pieces arranged to form an 18.5 in square with a 6.5 in square hole in the middle which was centered around the 6 in-diameter beampipe at $z=-81$ in, as shown in Fig 2.6. Early running required a hit in an identical array on the opposite side of the collision at $z=83$ in ("p counters"), but the information provided by these and by the alphabet counters was redundant, and the $p$ counters were removed when the new beampipe was installed.

Histograms of the ADC values for some of the counters are shown in Fig. 2.7. The trigger was designed to record events in which minimum-ionizing particles passed through the $\mathrm{B}, \mathrm{D}$, and pbar counters. [Any moderatelyrelativistic charged particle other than an electron is a minimum-ionizing particle (mip), and loses energy in a medium mainly through ionization. The amount of energy lost depends on the thickness of the material and the velocity of the particle; the mean energy lost over a given distance is 
described by the Bethe-Bloch equation [36].] Plots of the pbar ADC's show these mip peaks, which indicate the number of minimum-ionizing particles which passed through. These peaks are not seen clearly in the alphabet ADC's because of degradation of the scintillator, causing poorer correlation between the amount of light created by the mip track and that collected by the photomultiplier tubes; however, by increasing the voltage on the counters, triggering on minimum-ionizing particles was still possible. An entry in the $\log$ book from January 7, 1996 notes this, along with particular mention of the poor performance of the top $D$ counter.

A signal from the accelerator division reported the occurrence of a beam crossing at C0. At this signal, counting began in the TDC's, and the time when a charged particle was detected in the scintillator was recorded. If no charged particle was detected after $2^{11}$ counts, a hit was recorded in the last bin. Plots of the TDC values for the B, D, and Pbar counters (summed over all pieces of scintillator) are shown in Fig. 2.8. One TDC count is equivalent to $0.25 \mathrm{~ns}$ for all counters. However, the offsets for each counter are different due to differing lengths of cable, so that a particular TDC value does not correspond to the same amount of real time for all counters. Also shown in Fig. 2.8 is the TDC for the bottom D counter plotted against the TDC for one of the pbar counters. The dark region indicates the timing for beambeam collisions. Hits which occur earlier or later in one of the counters are due to something other than collision primaries. For example, a beam-gas 
interaction involving a proton and occurring before the proton bunch reaches the collision region could send particles into the pbar counters at times earlier than those for beam-beam collisions. Also, secondary interactions in the detector could cause hits at later times.

Since the counters on the detector side were behind the lead converter, we were able to trigger on events with a photon conversion regardless of the presence of charged tracks. In fact, the large frequency of interactions with the beampipe which sent particles into the detector resulted in many triggered collisions which produced no primary charged particles or photons in the acceptance.

The trigger rate during normal running conditions (D0 luminosity of $10^{30}$ $\mathrm{cm}^{-2} \mathrm{~s}^{-1}$ ) was around $300 \mathrm{~Hz}$. Only a fraction of the events which were triggered on were recorded; events were written at a rate of approximately $30 \mathrm{~Hz}$. The fraction of triggers not due to beam-beam collisions was less than about $5 \%$, and was mainly due to proton-induced beam-gas interactions.

Mean trigger rates and Tevatron running conditions for some of the January 1996 runs are given in Table 2.10. The BDpbar delay is an indirect measure of the time difference of signals from pbar and alphabet counters, and different values lead to triggers on beam-beam or beam-gas collisions. The $\mathrm{p}$ and pbar currents are the numbers of protons and anti-protons circulating in the ring. The raw trigger rate requires $A D C$ counts from the $\mathrm{B}, \mathrm{D}$, and pbar counters which are high enough to indicate the presence of 
collision-produced particles, and the beam trigger rate additionally requires coincidental signals from the TDC's of these counters. (The alphabet rate is not given because it was not recorded correctly in these runs.) The ratio of beam trigger rate to D0 luminosity seems to vary more in the low luminosity runs than in the runs with higher luminosity. The ratio of beam to raw rates is a measure of the amount of raw triggers due to beam-gas interactions. For the last few runs in Table 2.10 , the beam rate is reported as being higher than the raw rate, which is clearly impossible. The log book notes a "double pulsing" in the trigger rate beginning in run 1128, and during run 1137 , "beam trig / raw trig is ramping to 2.0 then back down over $\sim 5 \mathrm{~min}$ period - has occurred 4 times over last 200k events". The source of the problem is unclear.

The cross section seen by the MiniMax detector was estimated using the cross sections determined by CDF. These are [37] $80.03 \pm 2.24 \mathrm{mb}$ total cross section, $19.70 \pm 0.85 \mathrm{mb}$ elastic, and $9.46 \pm 0.44 \mathrm{mb}$ single diffractive (this is the sum of cross sections for diffractive dissociation of the proton and of the anti-proton). The non-single diffractive inelastic cross section is therefore $50.87 \mathrm{mb}$. Some single diffractive events (most likely with high diffractive mass) may have been triggered on, and not all double diffractive events pass the trigger. The cross section of events which actually passed the trigger was determined from the trigger rate and luminosity. The luminosity at $\mathrm{C} 0$ was taken as that at D0 corrected for differences in the magnetic architecture in 
the two regions and the fact that the bunches which collide at $\mathrm{C} 0$ are not the same pairs as those which collide at D0. The parameter $\beta$ is a measure of how tightly focussed the beams are at a given point. The ratio of the $\beta$ 's at $\mathrm{C} 0$ and at D0 is approximately $0.35 / 72$, and is further corrected by a factor of $1 / 0.7$ to take into account the variation of $\beta$ over the luminous region at D0. The ratio of trigger rate to D0 luminosity was typically around $0.30 \mathrm{mb}$. Therefore we estimate that the observed cross section was approximately $43 \mathrm{mb}$.

\subsubsection{Electromagnetic calorimeter}

A 28-module electromagnetic calorimeter was located behind the MWPC's, with the face at $z=282 \mathrm{in}$, centered on $x=8.49 \mathrm{in}, y=7.71 \mathrm{in}$. Twelve of the cells were $3.8 \times 3.8 \mathrm{in}^{2}$ with 30 layers each of $4.88 \mathrm{~mm}$-thick lead and $6 \mathrm{~mm}$ thick scintillator, which combined for a total length of about $27 \mathrm{X}_{0}$. These were made by the University of Michigan [38] and were used in Fermilab E-756. (Before 1995, the calorimeter consisted of 16 Michigan cells with the same total cross sectional area, and with the face at $z=288$ in.) Four $10 \mathrm{~cm} \times 10 \mathrm{~cm} \times 30 \mathrm{~cm}$ cells each had 4 photomultiplier tubes, which effectively made $165 \mathrm{~cm} \times 5 \mathrm{~cm} \times 30 \mathrm{~cm}$ modules. The cells, which were cut from longer hadronic calorimeter cells made by Wayne State University, were lead with $47 \times 47$ scintillating fibers of diameter $1 \mathrm{~mm}$ spaced $0.213 \mathrm{~cm}$ apart and running

the length of the module [39]. The effective radiation length was $0.78 \mathrm{~cm}$, 
so that the modules were about $38.5 \mathrm{X}_{0}$ long. The calorimeter was placed on a stand so that it also was rotated by $44.5^{\circ}$. The smaller cells occupied positions close to the beampipe in order to cover roughly the same area in $\eta-\phi$ space as the larger cells, as seen in Fig. 2.9 (recall that larger values of $\eta$ are closer to the beampipe at a given $z$ ). This is desirable since the density of particles is roughly uniform in lego space.

\subsubsection{Upstream tags}

In order to tag diffractive events, scintillator and hadron calorimetry was placed upstream of the collision point. A view of the upstream region is shown in Fig. 2.10. At $z \sim-25 \mathrm{~m}$, two hadron calorimeter modules, similar to the smaller cells of the electromagnetic calorimeter except having dimensions of $10 \mathrm{~cm} \times 10 \mathrm{~cm} \times 117 \mathrm{~cm}$, were positioned to detect leading anti-protons and anti-

neutrons. The machine magnets bent the $\overline{\mathrm{p}}$ 's with less-than-beam momentum by greater angles than the beam, and the $\bar{n}$ 's not at all. The anti-protons detected here had a Feynman $x$ of $x_{F} \simeq 0.5$, i.e. their momentum is about half that of the beam particles. Farther upstream at about $z=-60 \mathrm{~m}$, four scintillator counters were placed to detect showers from anti-protons with $x_{F} \sim 0.9$ that interact in what are called the kicker magnets. If at least one of these scintillator is hit, the event has a "ktag". Note that these counters did not only see diffractive anti-protons, but also products from beam-gas interactions in the upstream region. 


\subsection{Run history}

The concept of a detector with good coverage in the forward region which ultimately led to the MiniMax experiment originated in an initiative for a full-acceptance detector (FAD) [40] at the SSC. A proposal was later submitted for a maximum-acceptance experiment at Fermilab (MAX) [41] which was designed to investigate some of the physics goals of FAD. A small test/experiment with a detector covering only the forward region (MiniMax, Fermilab T-864) was approved in the spring of 1993. From late 1993 to early 1995, the MiniMax detector was installed, and collider data was taken with $8,12,16$, and finally 24 chambers in large-angle stereo. My work on the experiment began in September 1994.

The new beampipe was installed in February of 1995, and when the chambers were re-installed, they were given the new small-angle-stereo orientation with wires in 11 of the 24 chambers aligned parallel to each other for reasons discussed in the previous section. During the following five months, short opportunistic runs were taken with various thicknesses of lead and iron converter, and two different window thicknesses, in which a total of about $3.5 \times 10^{6}$ events were collected. The diffractive tags were commissioned in May and were available in more than $10^{6}$ events. The chambers were again removed and reconfigured in the fall of 1995 in order to compress them closer to the interaction point, away from some of the blinding pipe showers farther downstream, as will be discussed in Sec. 3.2.1. 
Production running in January of 1996 yielded $4.2 \times 10^{6}$ events, $2.7 \times 10^{6}$ of which had $1 \mathrm{X}_{0}$ lead in, and the remaining $1.5 \times 10^{6}$ events were run with the lead out. The luminosity in these runs was lower than in most earlier runs by about 1-2 orders of magnitude. This also led to fewer beam-gas interactions which, among other effects, meant that the sample of events with a ktag had a much higher fraction of diffractive events. Some information about the runs used in the analysis described in this work is given in Table 2.10.

The MiniMax detector was decommissioned the following spring. 
T864 PROJECTED VIEW

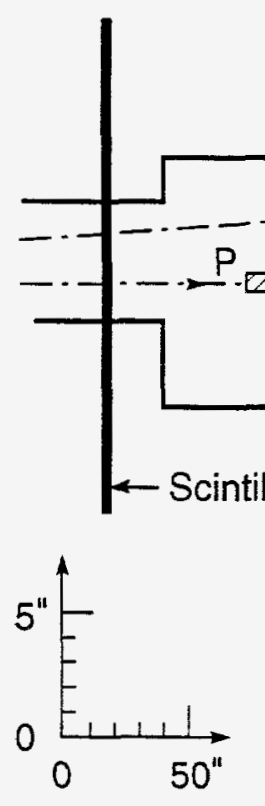

7839A5 $\quad 3-95$

\subsection{0 " Al Beampipes}



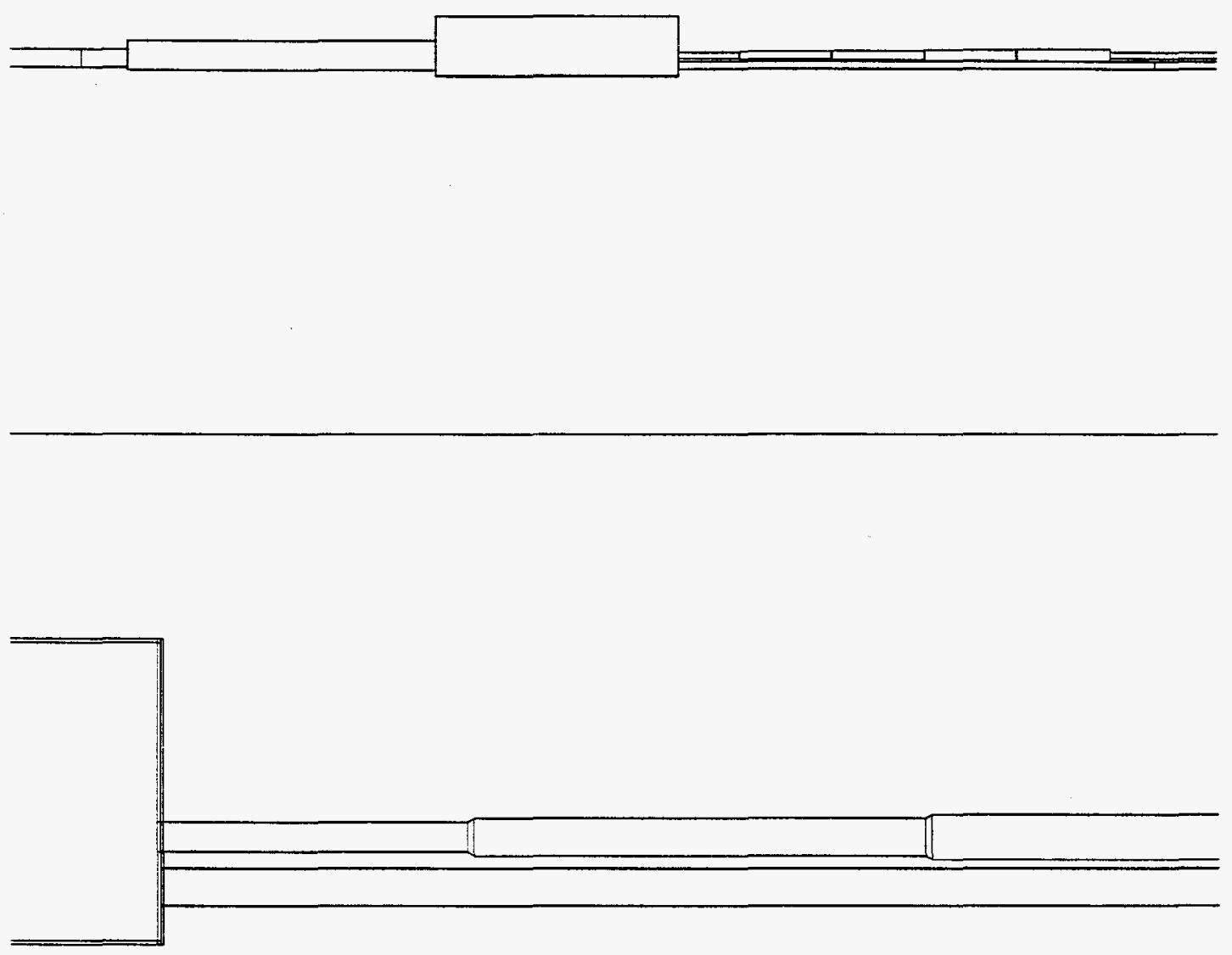

Figure 2.2: New Tevatron beampipe and abort at $\mathrm{C} 0$ and enlarged view of the region from $z \approx 110-190$ in. 

Figure 2.3: Acceptance in lego space. 
Figure 2.4: MWPC front view. 

Figure 2.5: MWPC top view. 


\section{$P \& \bar{P}$ Triggers}

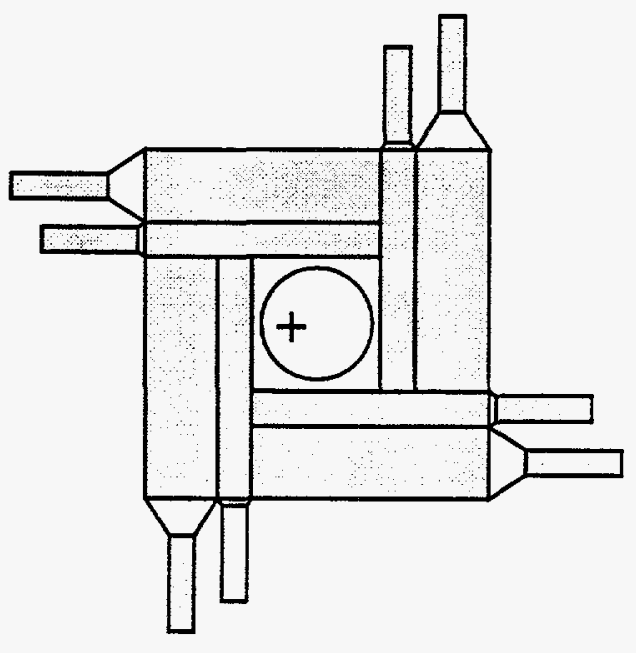

\section{A,B,C,D Triggers}
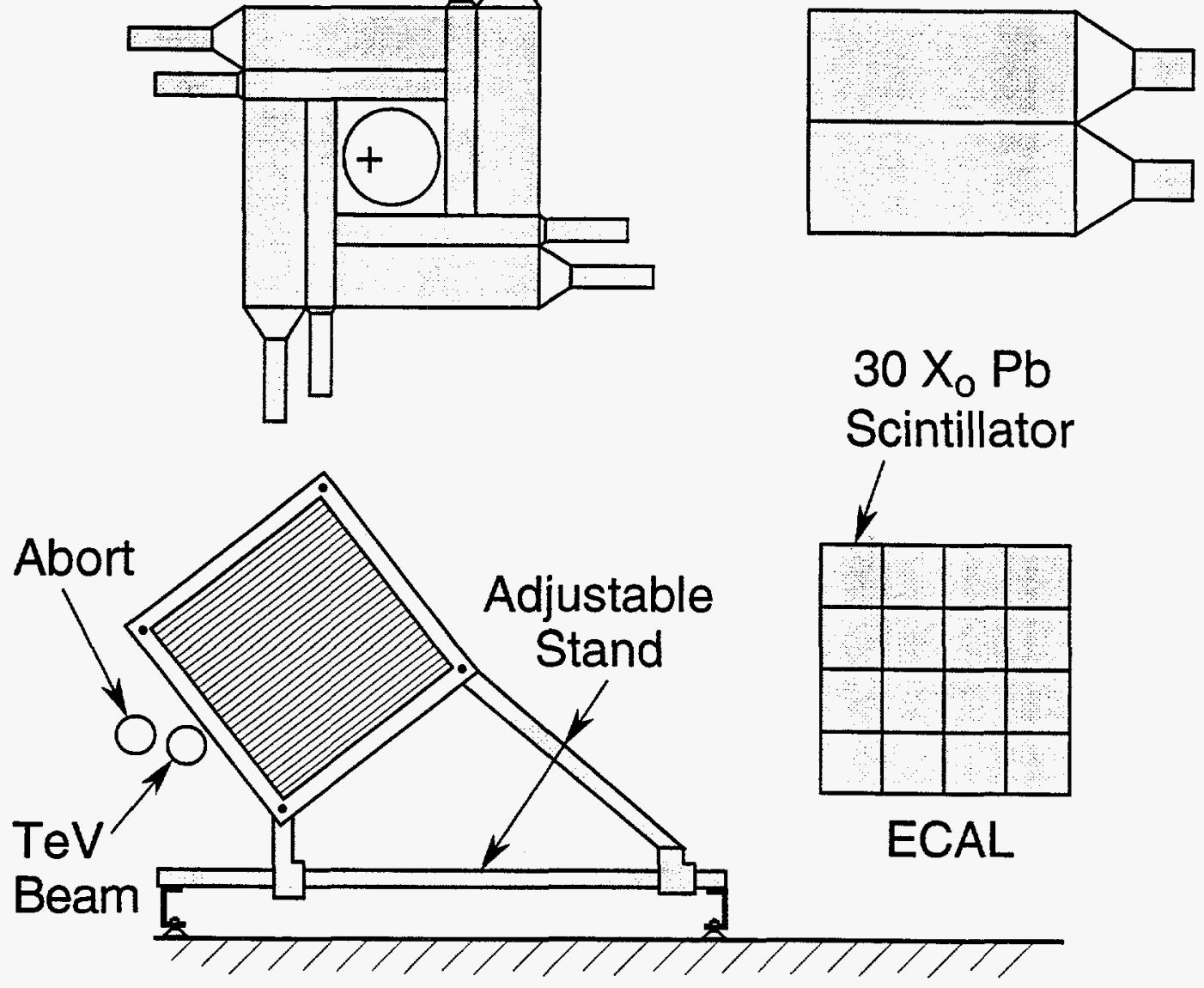

8-93

MWPC

7289A19

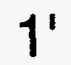

Figure 2.6: Pieces of apparatus including pbar and alphabet scintillator, chamber stands, and the original calorimeter. 

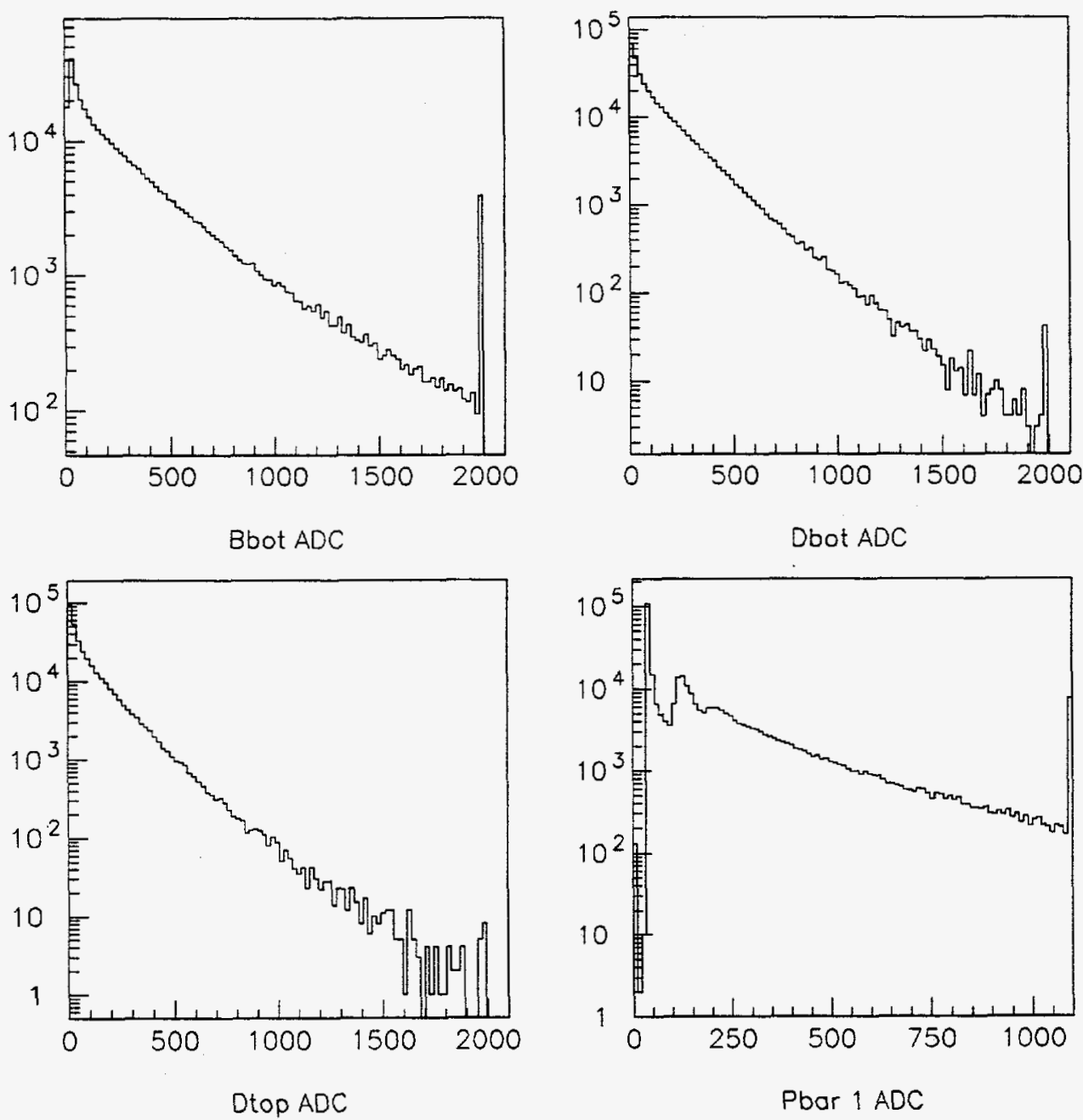

Figure 2.7: ADC spectra from some of the trigger counters. 


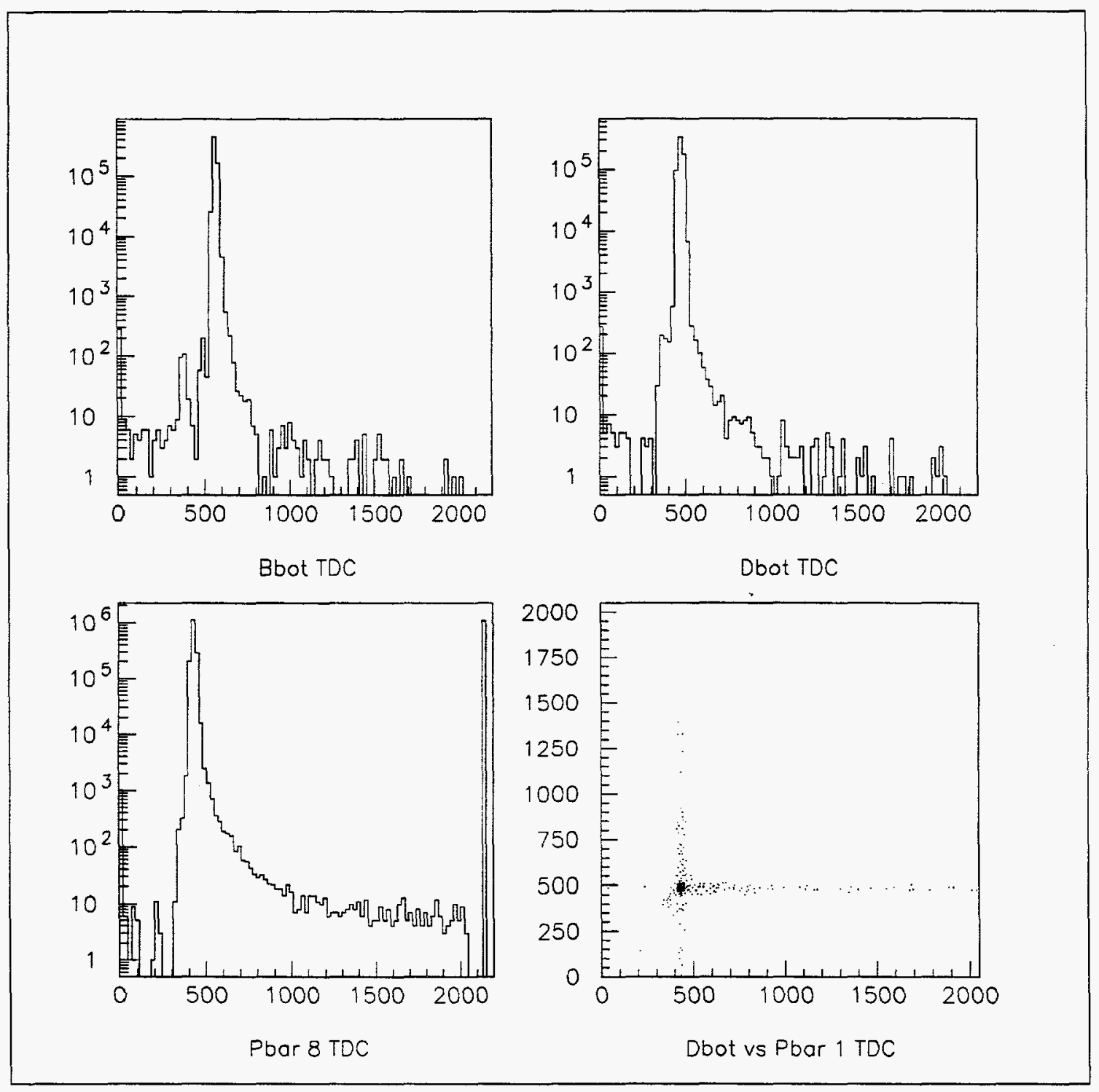

Figure 2.8: TDC spectra from some of the trigger counters. 


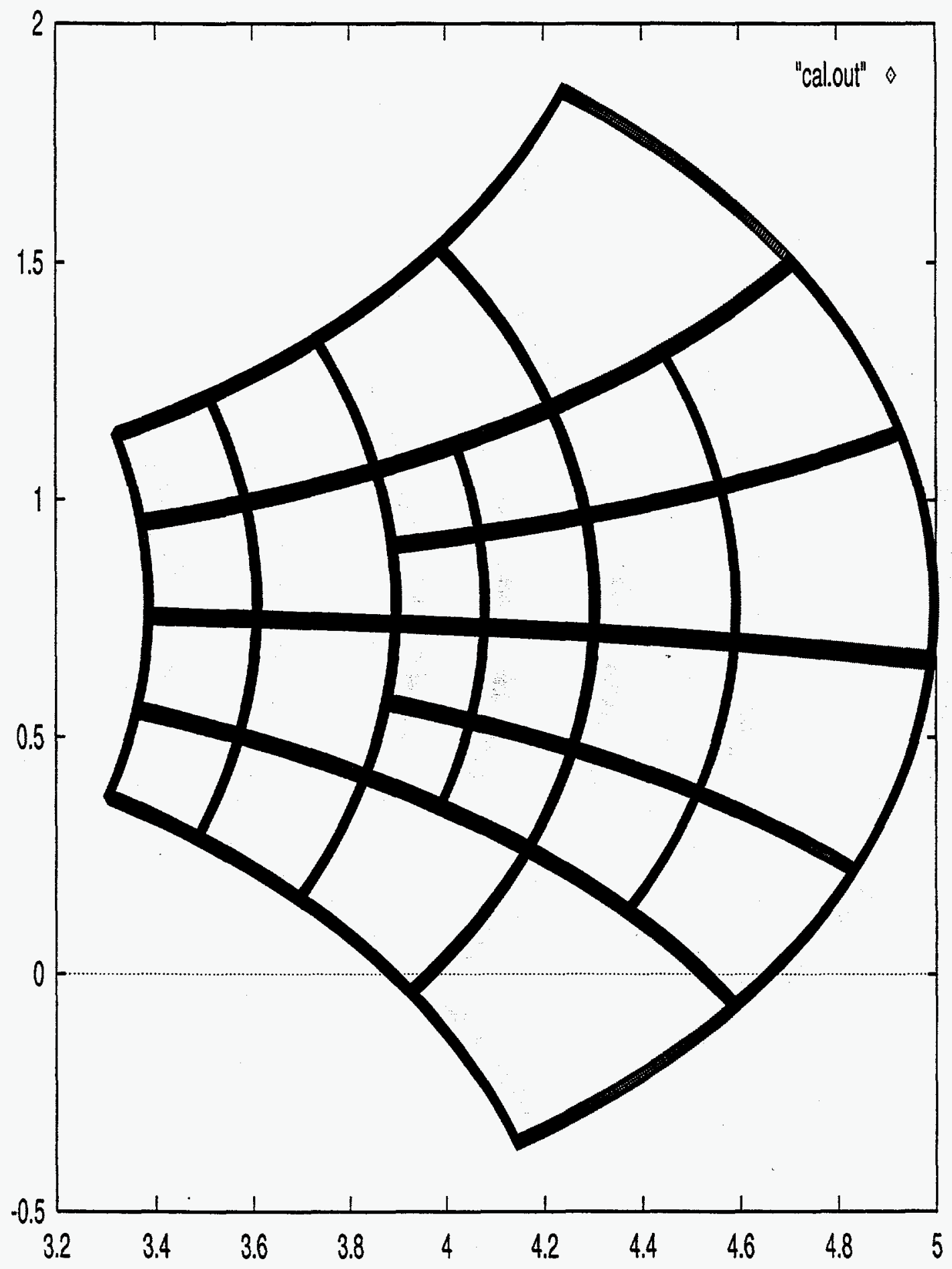

Figure 2.9: Electromagnetic calorimeter cells in lego space; the vertical axis is $\phi$ and the horizontal axis is $\eta$. 


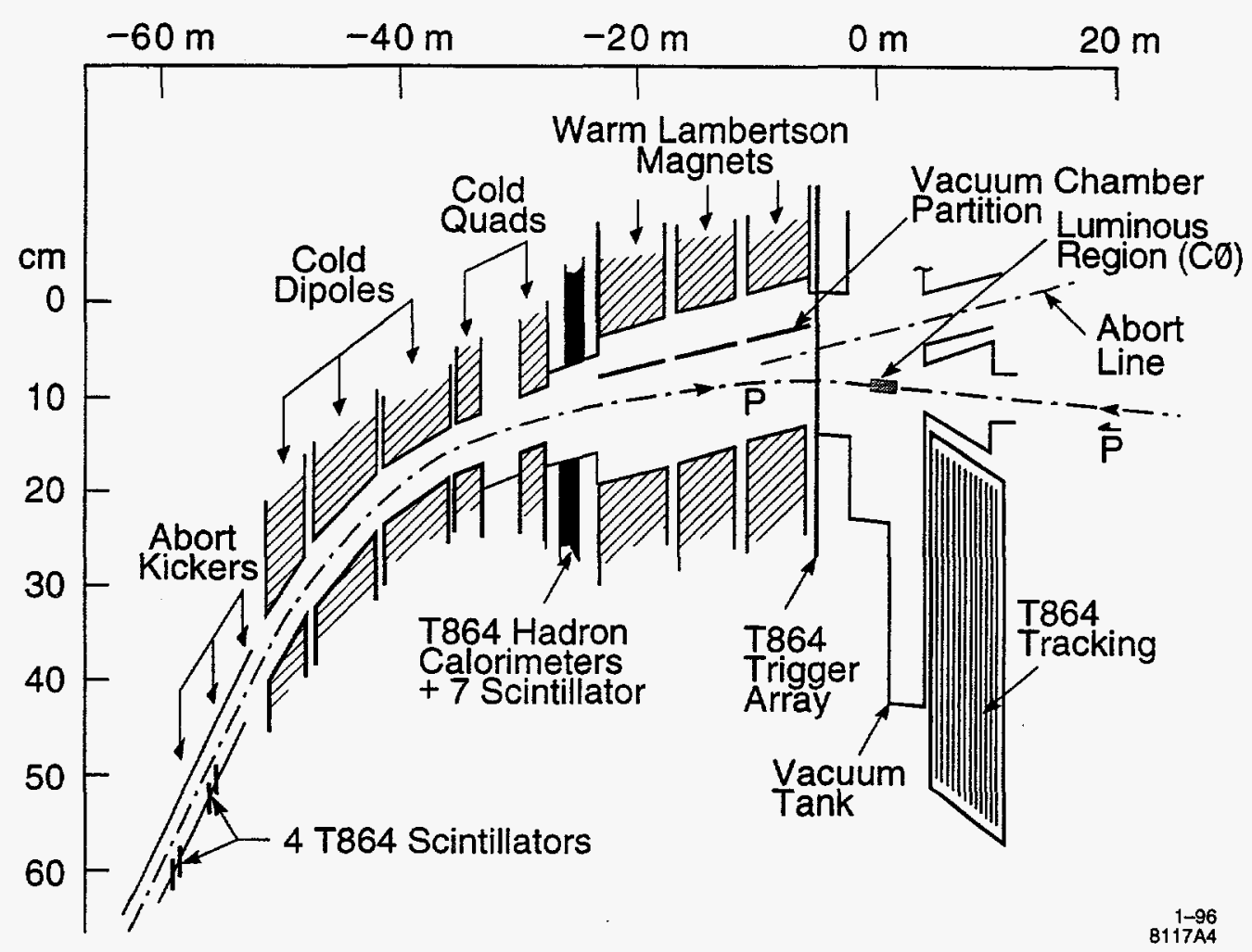

Figure 2.10: A view of the detector including the upstream region. 


\begin{tabular}{|c|c|c|c|c|c|c|c|}
\hline pipe & $z_{1}$ & $z_{2}$ & $\begin{array}{c}\text { diameter } \\
\text { (outer) }\end{array}$ & $x$ & $y$ & $\begin{array}{c}\text { thickness } \\
\text { (radial) }\end{array}$ & material \\
\hline Tev & -199.75 & -98. & 6. & -1.5 & 0.5 & 0.0625 & steel \\
Tev & -98. & 78. & 6. & -1.5 & 0.5 & 0.0625 & $\mathrm{Al}$ \\
Tev & 78. & 300. & 2.5 & 0. & 0. & 0.035 & $\mathrm{Al}$ \\
\hline abort & 78. & 130. & 2.5 & -3.0 & 1.0 & 0.035 & $\mathrm{Al}$ \\
abort & 130. & 300. & 2.5 & -3.0 & 1.0 & 0.065 & steel \\
\hline \hline & $z$ & inner & outer & $x$ & $y$ & thickness & material \\
& (center) & diameter & diameter & & & (in $z$ ) & \\
\hline flange & 78. & 0. & 8. & 2.0 & -1.5 & 0.5 & $\mathrm{Al}$ \\
\hline
\end{tabular}

Table 2.1: Old Tevatron beampipe and abort at $\mathrm{C}$. 


\begin{tabular}{|c|c|c|c|c|c|c|c|}
\hline pipe & $z_{1}$ & $z_{2}$ & $\begin{array}{c}\text { diameter } \\
\text { (outer) }\end{array}$ & $x$ & $y$ & $\begin{array}{c}\text { thickness } \\
\text { (radial) }\end{array}$ & material \\
\hline Tev & -199.75 & -75. & 6. & -1. & 1. & 0.0625 & steel \\
Tev & -75. & -60. & 6. & -1. & 1. & 0.0625 & $\mathrm{Al}$ \\
Tev & -60. & 40. & 10. & 0. & 0. & 0.25 & $\mathrm{Al}$ \\
Tev & 40. & 120. & 20. & 3. & 3. & 0.25 & $\mathrm{Al}$ \\
Tev & 120. & 139.942 & 2. & 0. & 0. & 0.03 & $\mathrm{Al}$ \\
Tev & 140.375 & 169.942 & 2.5 & 0. & 0. & 0.03 & $\mathrm{Al}$ \\
Tev & 170.375 & 199.942 & 3. & 0. & 0. & 0.03 & $\mathrm{Al}$ \\
Tev & 200.375 & 229.942 & 3.5 & 0. & 0. & 0.03 & $\mathrm{Al}$ \\
Tev & 230.375 & 260.375 & 4. & 0. & 0. & 0.03 & $\mathrm{Al}$ \\
Tev & 260.375 & 307. & 2. & 0. & 0. & 0.03 & $\mathrm{Al}$ \\
\hline abort & 120. & 275. & 2.5 & -3.264 & 1.088 & 0.0625 & $\mathrm{Al}$ \\
abort & 275. & 307. & 2.5 & -3.264 & 1.088 & 0.0625 & steel \\
\hline \hline & $z$ & inner & outer & $x$ & $y$ & thickness & $\mathrm{material}$ \\
& & diameter & diameter & offset & offset & (in $z$ ) & \\
\hline flange & -60. & 0. & 10. & 0. & 0. & 0.25 & $\mathrm{Al}$ \\
face plate & 40. & 0. & 20. & 3. & 3. & 0.375 & $\mathrm{Al}$ \\
face plate & 120. & 0. & 20. & 3. & 3. & 0.375 & $\mathrm{Al}$ \\
"window" & 120. & 0. & 9. & 3.8891 & 3.8891 & 0.25 & $\mathrm{Al}$ \\
flare & 140.1585 & 2. & 2.5 & 0. & 0. & 0.03 & $\mathrm{Al}$ \\
flare & 170.1585 & 2.5 & 3. & 0. & 0. & 0.03 & $\mathrm{Al}$ \\
flare & 200.1585 & 3. & 3.5 & 0. & 0. & 0.03 & $\mathrm{Al}$ \\
flare & 230.1585 & 3.5 & 4. & 0. & 0. & 0.03 & $\mathrm{Al}$ \\
flare & 260.375 & 2. & 4. & 0. & 0. & 0.03 & $\mathrm{Al}$ \\
\hline
\end{tabular}

Table 2.2: New Tevatron beampipe and abort at $\mathrm{C} 0$. 


\begin{tabular}{|c|c|c|c|c|c|}
\hline chamber & angle & $x_{0}$ & $y_{0}$ & $z_{0}$ & readout \\
\hline 1 & 1.70504 & 9.38306 & -1.96032 & 173.993 & nanometrics \\
2 & 3.01962 & 15.9741 & 3.55498 & 178.014 & nanometrics \\
3 & 1.18197 & 4.19295 & 1.63239 & 181.974 & nanometrics \\
4 & 2.49308 & 11.5763 & 2.44270 & 185.996 & nanometrics \\
5 & -2.23082 & 13.6522 & 9.03440 & 203.816 & Michigan \\
6 & 1.96328 & 10.8733 & -0.442493 & 208.952 & Michigan \\
7 & -3.03528 & 14.9853 & 6.86178 & 212.912 & Michigan \\
8 & 1.41509 & 7.64913 & 1.50400 & 216.934 & Michigan \\
9 & -0.400473 & 4.92871 & 10.5747 & 247.129 & Michigan \\
10 & -2.21307 & 14.7361 & 13.0676 & 250.841 & Michigan \\
11 & 2.23920 & 14.9585 & 3.01641 & 256.658 & Michigan \\
12 & -2.74165 & 16.9554 & 10.4961 & 260.679 & Michigan \\
\hline
\end{tabular}

Table 2.3: Chamber alignment from 2/16/94. The location and orientation of each chamber is defined by the $\left(x_{0}, y_{0}, z_{0}\right)$ coordinates of the midpoint of wire number zero, together with the angle with respect to the $x$-axis of the vector pointing in the direction of increasing wire number. 


\begin{tabular}{|c|c|c|c|c|c|}
\hline chamber & angle & $x_{0}$ & $y_{0}$ & $z_{0}$ & readout \\
\hline 101 & -0.5990 & 1.017 & 10.103 & 122.83 & Michigan \\
102 & -2.1730 & 10.135 & 12.038 & 126.70 & Michigan \\
103 & -0.9782 & 2.762 & 11.960 & 130.83 & Michigan \\
104 & -2.5529 & 11.810 & 10.092 & 134.70 & Michigan \\
105 & 2.9449 & 14.174 & 6.474 & 155.33 & nanometrics \\
106 & -1.7676 & 9.055 & 13.945 & 157.77 & nanometrics \\
107 & -1.3747 & 6.559 & 14.038 & 161.52 & nanometrics \\
108 & 0.2038 & 1.371 & 6.457 & 165.52 & nanometrics \\
109 & -0.5331 & 1.466 & 9.924 & 184.70 & nanometrics \\
110 & -2.1038 & 10.046 & 12.062 & 188.70 & nanometrics \\
111 & -0.8795 & 2.601 & 11.817 & 192.70 & nanometrics \\
112 & 0.6992 & 1.246 & 2.655 & 196.70 & nanometrics \\
113 & 2.8384 & 13.312 & 5.131 & 203.27 & Michigan \\
114 & -1.8662 & 9.191 & 13.135 & 207.02 & Michigan \\
115 & 2.0755 & 10.282 & 1.746 & 212.83 & Michigan \\
116 & 0.5036 & 1.530 & 4.230 & 216.83 & Michigan \\
117 & 2.9981 & 15.017 & 7.409 & 228.02 & nanometrics \\
118 & -1.6949 & 9.255 & 14.488 & 232.02 & nanometrics \\
119 & 0.2893 & 2.138 & 6.258 & 236.02 & nanometrics \\
120 & -1.2743 & 6.283 & 14.190 & 241.58 & nanometrics \\
121 & -1.4826 & 7.788 & 14.272 & 246.58 & Michigan \\
122 & -3.0389 & 14.772 & 8.508 & 251.89 & Michigan \\
123 & -0.7090 & 3.398 & 12.185 & 254.58 & Michigan \\
124 & -2.2739 & 12.464 & 12.906 & 256.14 & Michigan \\
\hline
\end{tabular}

Table 2.4: Chamber alignment from 11/23/94. 


\begin{tabular}{|c|c|c|c|c|c|}
\hline chamber & angle & $x_{0}$ & $y_{0}$ & $z_{0}$ & readout \\
\hline 101 & -1.0477 & 3.230 & 11.849 & 132.65 & Michigan \\
102 & -2.6107 & 11.972 & 9.215 & 136.53 & Michigan \\
103 & -0.7886 & 1.675 & 11.045 & 140.65 & Michigan \\
104 & -2.3572 & 10.755 & 10.889 & 144.53 & Michigan \\
105 & -2.3554 & 10.506 & 11.239 & 163.59 & nanometrics \\
106 & -0.5289 & 1.512 & 9.405 & 167.59 & nanometrics \\
107 & -2.0917 & 10.552 & 11.886 & 171.59 & nanometrics \\
108 & 0.7801 & 1.752 & 2.136 & 175.59 & nanometrics \\
109 & 0.7874 & 1.680 & 2.368 & 194.53 & Michigan \\
110 & -2.1169 & 10.376 & 12.193 & 198.41 & Michigan \\
111 & -2.3591 & 11.156 & 11.490 & 202.28 & Michigan \\
112 & 0.5364 & 1.687 & 3.299 & 206.53 & Michigan \\
113 & -2.3558 & 11.167 & 11.409 & 212.84 & nanometrics \\
114 & -2.5271 & 12.242 & 10.461 & 216.84 & nanometrics \\
115 & -2.3580 & 12.240 & 11.516 & 221.09 & nanometrics \\
116 & 0.9668 & 3.447 & 1.569 & 226.66 & nanometrics \\
117 & -2.3592 & 11.679 & 11.749 & 237.72 & nanometrics \\
118 & -2.2361 & 11.216 & 12.239 & 241.72 & nanometrics \\
119 & 0.7847 & 2.616 & 2.686 & 245.84 & nanometrics \\
120 & -2.4771 & 12.387 & 11.188 & 249.72 & nanometrics \\
121 & -2.2556 & 11.497 & 12.241 & 256.15 & Michigan \\
122 & 0.7104 & 2.377 & 3.154 & 260.15 & Michigan \\
123 & 0.7801 & 2.779 & 2.820 & 264.40 & Michigan \\
124 & 0.7818 & 2.775 & 2.816 & 265.97 & Michigan \\
\hline
\end{tabular}

Table 2.5: Chamber alignment with 11 chambers parallel from 3/19/95. 


\begin{tabular}{|c|c|c|c|c|c|}
\hline chamber & angle & $x_{0}$ & $y_{0}$ & $z_{0}$ & readout \\
\hline 101 & -1.0315 & 2.806 & 11.653 & 123.69 & Michigan \\
102 & -2.3422 & 10.117 & 11.032 & 126.57 & Michigan \\
103 & -0.7575 & 0.853 & 10.870 & 129.69 & Michigan \\
104 & -2.5953 & 11.792 & 9.255 & 132.57 & Michigan \\
105 & -2.3318 & 9.997 & 10.910 & 135.69 & nanometrics \\
106 & -0.5044 & 0.818 & 9.193 & 138.69 & nanometrics \\
107 & -2.0857 & 10.076 & 11.700 & 141.69 & nanometrics \\
108 & 0.8029 & 1.252 & 1.813 & 144.69 & nanometrics \\
109 & 0.7959 & 1.692 & 2.568 & 167.09 & Michigan \\
110 & -2.1084 & 10.090 & 12.305 & 169.89 & Michigan \\
111 & -2.3370 & 10.970 & 11.702 & 172.84 & Michigan \\
112 & 0.5794 & 1.535 & 3.508 & 176.09 & Michigan \\
113 & -2.3370 & 11.199 & 11.781 & 178.84 & nanometrics \\
114 & -2.5063 & 12.360 & 10.771 & 181.84 & nanometrics \\
115 & -2.3405 & 11.424 & 11.812 & 185.09 & nanometrics \\
116 & 0.9669 & 3.366 & 1.695 & 189.65 & nanometrics \\
117 & -2.3370 & 11.602 & 12.036 & 194.29 & nanometrics \\
118 & -2.2201 & 11.327 & 12.524 & 197.29 & nanometrics \\
119 & 0.8029 & 2.814 & 2.963 & 200.29 & nanometrics \\
120 & -2.4574 & 12.648 & 11.415 & 203.29 & nanometrics \\
121 & -2.3527 & 12.056 & 12.114 & 206.16 & Michigan \\
122 & 1.0420 & 3.560 & 1.613 & 209.16 & Michigan \\
123 & 0.7837 & 3.140 & 3.048 & 212.41 & Michigan \\
124 & 0.6266 & 2.681 & 3.748 & 215.41 & Michigan \\
\hline
\end{tabular}

Table 2.6: Chamber alignment after compression from 3/24/96. 


\begin{tabular}{|c|c|c|c|c|c|c|}
\hline counter & $x$ & $y$ & $z$ & height & width & angle \\
\hline A & -2.8 & -2.2 & 108. & 5.5 & 5.5 & 0.26 \\
B1 & -5.9 & -3.7 & 155. & 8.0 & 16.0 & 0.26 \\
B2 & -3.8 & 3.9 & 155. & 8.0 & 16.0 & 0.26 \\
C1 & -5.9 & -3.7 & 223. & 8.0 & 16.0 & 0.26 \\
C2 & -3.8 & 3.9 & 223. & 8.0 & 16.0 & 0.26 \\
D1 & -5.9 & -3.7 & 269. & 8.0 & 16.0 & 0.26 \\
D2 & -3.8 & 3.9 & 269. & 8.0 & 16.0 & 0.26 \\
E & -12.2 & -4.0 & 322. & 13.0 & 13.0 & 1.04 \\
\hline
\end{tabular}

Table 2.7: Configuration of alphabet counters for runs with the old beampipe, through 1994.

\begin{tabular}{|c|c|c|c|c|}
\hline counter & $x$ & $y$ & $z$ & angle \\
\hline A & & & & \\
B1 & 5.1 & 10.8 & 189. & 45. \\
B2 & 10.8 & 5.1 & 189. & 45. \\
C1 & 5.0 & 10.7 & 180. & 45. \\
C2 & 10.7 & 5.0 & 180. & 45. \\
D1 & 6.7 & 11.3 & 280. & 41.7 \\
D2 & 12.0 & 5.3 & 280. & 41.7 \\
E & -12.2 & -4.0 & 322. & 0. \\
\hline
\end{tabular}

Table 2.8: Configuration of alphabet counters during running with the new beampipe from $2 / 95-7 / 95$.

\begin{tabular}{|c|c|c|c|c|}
\hline counter & $x$ & $y$ & $z$ & angle \\
\hline A & 2.8 & 2.8 & 264. & 45. \\
B1 & 6.1 & 11.8 & 279. & 45. \\
B2 & 11.8 & 6.1 & 279. & 45. \\
C1 & 6.0 & 11.7 & 270. & 45. \\
C2 & 11.7 & 6.0 & 270. & 45. \\
D1 & 7.2 & 7.6 & 157. & 27.4 \\
D2 & 10.9 & 0.5 & 157. & 27.4 \\
E & -12.2 & -4.0 & 322. & 0.0 \\
\hline
\end{tabular}

Table 2.9: Configuration of alphabet counters during runs with compressed MWPC telescope. 


\begin{tabular}{|c|c|c|r|c|c|c|c|r|r|c|c|}
\hline $\begin{array}{c}\text { run } \\
\text { number }\end{array}$ & date & lead & $\begin{array}{r}\text { number } \\
\text { events }\end{array}$ & $\begin{array}{c}\text { BDpbar } \\
\text { delay } \\
(\mathrm{ns})\end{array}$ & $\begin{array}{c}\mathrm{p} \\
\text { current } \\
\left(10^{9}\right)\end{array}$ & $\begin{array}{c}\text { pbar } \\
\text { current } \\
\left(10^{9}\right)\end{array}$ & $\begin{array}{c}\text { D0 } \\
\text { luminosity } \\
\left(10^{30} \mathrm{~cm}^{-2} \mathrm{~s}^{-1}\right)\end{array}$ & $\begin{array}{r}\text { raw } \\
\text { rate } \\
(\mathrm{Hz})\end{array}$ & $\begin{array}{r}\text { beam } \\
\text { rate } \\
(\mathrm{Hz})\end{array}$ & $\begin{array}{c}\text { beam/ } \\
\text { D0lum } \\
(\mathrm{mb})\end{array}$ & $\begin{array}{c}\text { beam/ } \\
\text { raw }\end{array}$ \\
\hline 1089 & $1 / 18$ & out & 50112 & 8 & 105. & 83. & 0.91 & 269. & 258. & 0.28 & 0.96 \\
1093 & $1 / 18$ & out & 103061 & 8 & 95. & 79. & 0.63 & 192. & 190. & 0.30 & 0.99 \\
1096 & $1 / 18$ & in & 249986 & 8 & 38. & 30. & 0.14 & 43. & 42. & 0.30 & 0.97 \\
1099 & $1 / 18$ & in & 174775 & 8 & 37. & 29. & 0.09 & 32. & 32. & 0.33 & 0.99 \\
1103 & $1 / 19$ & in & 67117 & 8 & 34. & 27. & 0.05 & 18. & 18. & 0.35 & 0.98 \\
1104 & $1 / 19$ & out & 50461 & 8 & 34. & 26. & 0.04 & 13. & 13. & 0.36 & 0.98 \\
1108 & $1 / 19$ & out & 249990 & 8 & 37. & 30. & 0.15 & 45. & 45. & 0.31 & 0.99 \\
1109 & $1 / 19$ & in & 250081 & 8 & 36. & 29. & 0.11 & 34. & 34. & 0.32 & 0.99 \\
1110 & $1 / 20$ & in & 249967 & 8 & 35. & 28. & 0.08 & 27. & 26. & 0.32 & 0.98 \\
1111 & $1 / 20$ & out & 234361 & 8 & 34. & 27. & 0.06 & 20. & 19. & 0.33 & 0.98 \\
1123 & $1 / 21$ & out & 37126 & 8 & 61. & 74. & 0.45 & 128. & 128. & 0.29 & 1.00 \\
1124 & $1 / 21$ & out & 91958 & 8 & 21. & 14. & 0.05 & 13. & 13. & 0.28 & 1.00 \\
1125 & $1 / 21$ & in & 251434 & 8 & 20. & 13. & 0.03 & 9. & 9. & 0.36 & 1.00 \\
1126 & $1 / 22$ & in & 47944 & 8 & 19. & 13. & 0.02 & 8. & 7. & 0.35 & 1.00 \\
1127 & $1 / 22$ & in & 28933 & 6 & 19. & 13. & 0.01 & 3. & 3. & 0.38 & 0.99 \\
1129 & $1 / 22$ & out & 53767 & 10 & 70. & 85. & 0.55 & 195. & 204. & 0.37 & 1.05 \\
1132 & $1 / 22$ & out & 52402 & 4 & 68. & 80. & 0.41 & 138. & 146. & 0.36 & 1.06 \\
1137 & $1 / 22$ & in & 249977 & 8 & 65. & 74. & 0.26 & 128. & 137. & 0.52 & 1.07 \\
1139 & $1 / 23$ & in & 153014 & 8 & 60. & 67. & 0.16 & 75. & 74. & 0.46 & 1.00 \\
\hline
\end{tabular}

Table 2.10: Running conditions from some January 1996 runs. 


\section{Chapter 3}

\section{Simulations}

\subsection{PYTHIA}

Minimum-bias collisions at $\sqrt{s}=1.8 \mathrm{TeV}$ are simulated using PYTHIA version 5.702 and JETSET $7.401[42,43]$. The combination of PYTHIA and JETSET provide a commonly-used event generator for high-energy collisions of elementary particles based on the parton model [44], and will be hereafter referred to as "PYTHIA". The physics of the collisions that PYTHIA deals with includes

the parton distribution functions of the beam particles, i.e., the flavor of the quarks and the fraction of energy carried by the constituent quarks and gluons of the incident proton and anti-proton, any radiation from the beam particles, such as the emission of a gluon 
from a quark, which leads to an initial-state shower,

the hard process between the incoming partons from each shower which produces outgoing particles,

any final-state showers produced by outgoing partons,

the beam remnants, which must return to color-neutral states after

losing the interacting partons,

the hadronization of outgoing partons - confinement requires that they form color-neutral hadrons,

and the decay of any unstable outgoing particles.

For the hadronization, PYTHIA uses a string fragmentation model, specifically what is called the Lund model [45]. Its basis is a string connecting a quark and an anti-quark which stretches as the partons move away from each other until it breaks by creating a new quark-anti-quark pair. This continues until only on-mass-shell hadrons remain, where a hadron is a color-singlet pair connected by a small piece of string.

Default values are taken for all parameters except that particles with a mean invariant lifetime $c \tau$ greater than $1 \mathrm{~cm}$ are not decayed. This allows the decays of $\mathrm{K}_{s}^{0}$ and $\Lambda^{0}$ particles to be studied later.

For non-single diffractive inelastic $\mathrm{p} \overline{\mathrm{p}}$ collisions at $1.8 \mathrm{TeV}, \mathrm{PYTHIA}$ gives mean numbers of particles into the acceptance per event of $0.61 \pi^{ \pm}$'s, 0.15 other charged particles, and $0.70 \gamma^{\prime}$ 's. The inclusive pseudorapidity distri- 
butions, $d N / d \eta$, for charged pions, all charged particles, and photons are shown in Fig. 3.1. (An interesting note is that the larger fraction of particles with $6<\eta<10$ in the charged distribution relative to the charged-pion distribution is due to leading protons and anti-protons from double diffractive processes.)

The cross sections for various types of events included in the minimumbias events generated by PYTHIA are given in Table 3.1. Note that compared to the cross sections reported by CDF (Sec. 2.2), PYTHIA underestimates the total and elastic cross sections, and overestimates the single diffractive.

\subsection{GEANT}

The particles generated in a collision are then taken as input into a GEANT simulation (GEANT version 3.21 [46]) where they are propagated through the material of the detector. The initial position of the collision primary particles is taken to be $u=v=0$, and $z$ given by a Gaussian distribution with a mean of 7 in and standard deviation $16.2 \mathrm{in}$, in order to reproduce the variance of the collision point seen in the data. Cross sections and simulations of processes such as hadronic interactions, electromagnetic processes, ionization by charged particles, multiple scattering, and decays in flight are included in GEANT. Default routines are used for all such processes, as well as the default energies below which particles are not tracked ( $1 \mathrm{MeV}$ for photons and electrons, $10 \mathrm{MeV}$ for hadrons and muons). The energy deposited in material 
due to interactions is given at each step, and the secondary particles produced are added to the list of particles to be tracked through the detector.

The energy deposited in the detector elements is used to model the response to particle interactions seen in the real data. At least $4.4(2.2) \mathrm{MeV}$ is required to be deposited in the 1 in ( 0.5 in)-thick scintillator trigger counters in order to keep the event. Figure 3.2 a shows the energy deposited in the 1 in-thick scintillator counters for minimum bias events, and Fig. 3.3 a shows the energy deposited by single charged pions (minimum-ionizing tracks). In Fig. $3.2 \mathrm{a}$, the peak for one minimum-ionizing particle passing through the scintillator can be seen around $4.5 \mathrm{MeV}$, and the smaller peak for two mip's at $9-10 \mathrm{MeV}$. The first minimum-ionizing peak starts at about $4 \mathrm{MeV}$, and has a maximum at $4.5 \mathrm{MeV}$. The low energy background is fairly insignificant. Therefore, any cutoff energy between these values is reasonable. The value $4.4 \mathrm{MeV}$ is chosen to be as large as possible in order to increase the mean number of wires hit in the MWPC's ("NHITS") for triggered events without missing a significant part of the mip peak. (The reason for this will be given in Sec. 3.2.1.)

For the GEANT simulation, the gas in each MWPC is segmented into 128 pieces corresponding to the volume which surrounds each wire in the actual chambers. The wires are not included in the simulation. Plots similar to those for the scintillator energy are given for the chamber pulse heights (energy deposited in a segment) in Fig. 3.2 b and Fig 3.3 b. For single charged 
pions, the low energy background which falls off around $0.3-0.4 \mathrm{keV}$ where the mip peak begins is presumably due to tracks which pass through more than one segment and deposit some fraction of the minimum-ionizing energy in each segment. For minimum-bias events, the low energy background is much higher and extends into the minimum-ionizing peak. The cutoff value chosen to represent a hit wire is $0.4 \mathrm{keV}$, where the background falls to a level comparable to the mip contribution. Note that in the actual MWPC's, ionized electrons created by the charged tracks drift to the positively charged wires and the amount of charge collected on a wire determines the pulse height. This may not be linearly related to the energy deposited in the gas; however, direct comparisons of pulse heights in the GEANT and in the data discussed in Sec. 3.2.1 show that this is not an unreasonable assumption.

The energy deposited in each cell of the lead-scintillator electromagnetic calorimeter is recorded, so that it can be used to calibrate the signals from the actual calorimeter.

The other crucial detector element which is included is converter of various thicknesses.

Because of the large observed background of particles due to interactions in material in the forward (detector) region, many objects not related to the detector are included in the simulation. These include the Tevatron beam pipe and abort pipe, the main ring and its abort, support stands, vacuum pumps, the concrete floor, etc. In spite of all these additions, the mean 
number of wires hit in each MWPC is lower than that in the real data by about a factor of two (see Figs. 3.4, 3.5).

\subsubsection{GEANT NHITS study}

The NHITS distribution is dependent on the pulse-height and trigger cuts. A lower pulse-height cut allows lower-energy hits to be counted as hits by actual tracks, thus increasing the NHITS in each event. A higher cut on energy deposited in the scintillator counters in order to trigger (i.e. to keep that event) tends to cut out events with less energy or number of tracks going into the detector, so that the mean NHITS of the remaining events is higher than that for all events. The cuts made on the GEANT data are intended to match those in the real data, namely to make cuts based on the signatures of real charged tracks going through the chambers and scintillator. However, we tried varying these cuts through all reasonable (and even unreasonable) values to see if the NHITS distribution of the data could be reproduced by the GEANT simulation. Only 12 of the 24 MWPC's read out pulse-height information, so we use only those chambers in the following analysis. The pulse height is calibrated in terms of charge deposited on the wires, not energy deposited in the gas as it is in GEANT, and is not the same in all chambers. The first step, therefore, is to adjust the energy scales of the chambers in the GEANT so that the mean of a Gaussian fit to the peaks of both the real and GEANT pulse-height distributions are equivalent. The 
result is shown in Figs. 3.6, 3.7. The mean NHITS in these 12 chambers is 93 for run 867 . It is clear in Table 3.2 that there is no combination of pulse-height and scintillator cuts which gives a mean NHITS this high in the GEANT data. For the lowest possible pulse-height cut (pulse height $>0$ ), and the highest shown cut on energy deposited in the scintillator ( $8 \mathrm{MeV}$, which practically requires two charged tracks rather than one), the mean NHITS in those 12 chambers is only 79.

The next attempts to find an explanation for the lower NHITS in GEANT include the following:

1. Increasing the thickness of the beampipe from 0.03 in to 0.035 in to check for GEANT problems with the boundary;

2. lowering the energy thresholds of particles for GEANT to track to $1 / 2$ the default value;

3. changing the defaults of GEANT so that delta rays are produced;

4. supposing that the collision point is not where we think it is relative to the pipe, and therefore changing the $z$ of the collision point to 10 in closer to the pipe, or changing $x$ and $y$ by 1 in each towards the pipe.

None of these have much effect on the NHITS. Next we tried adding the main ring and correcting the description of the abort pipe to include the change of material to steel at $z>275$ in, which increased the mean NHITS by about $20 \%$. Encouraged by this, we added more of the material nearby the detector: 
2ft-thick concrete floor, tunnel wall, chamber stands, Tevatron-beampipe and main-ring support stands, and vacuum ion pumps.

The effect on NHITS of material close to the wire chambers was studied in more detail by removing that material. The beampipe is the largest source of non-collision-point tracks hitting the chambers (Fig. 3.8). The abort pipe and the G-10 frames of the wire chambers were also studied (Figs. 3.9, 3.10). The rear chambers are hit by tracks from these sources more than the front; in fact, when all three of the sources are removed, the mean number of hits per event in each chamber is approximately a constant between 3.5 and 4 (Fig. 3.11), as opposed to the roughly linear increase in hits going from about 4 in the front chamber to more than 8 in the last, as in Fig. 3.4. (This is, of course, for lead-out runs. For lead-in runs, the number of hits in the chambers behind the lead is much greater than that in the front due to conversions of primary photons.) In the real data, the number of hits per chamber increases faster than linearly towards the rear of the detector. The addition of the beampipe supports in the GEANT simulation reproduces this effect fairly well. These observations are what led to the compression of the wire chambers which moved them closer to the collision point before the production running.

It has been noted [47] that the multiplicity distributions of low- $p_{T}$ charged particles in PYTHIA were incorrectly extrapolated from collider data at high $p_{T}$. Since the MiniMax detector is more sensitive than conventional collider 
detectors to low- $p_{T}$ particles, these can potentially contribute significantly to the NHITS. We tried using HERWIG, another commonly-used event generator (especially for jet studies) to see if the NHITS distribution might be better reproduced. Comparisons of $d N / d \eta$ from non-diffractive PYTHIA events and HERWIG events show that the mean number of particles produced is higher for PYTHIA (see Fig. 3.12 for $d N_{c h} / d \eta$ ). The NHITS distribution obtained using HERWIG was not significantly different.

Another attempt to increase the mean NHITS in the simulations was to change the defaults for multiple interactions in PYTHIA. The occurrence of hard interactions of more than one parton pair in a hadronic collision is not well understood, and PYTHIA provides several models. The default is that the probability of multiple interactions is equal for all events, with a sharp $p_{\perp \min }$ cut-off. It was suggested to us that the option with "multiple interactions assuming a varying impact parameter and a hadronic matter overlap consistent with a double Gaussian matter distribution ... with a continuous turn-off of the cross section at $p_{\perp 0}$ " (Ref. [43], p. 222) would give larger multiplicity fluctuations, and therefore possibly larger mean NHITS. The effect of using this model is also negligible.

A study was done by the ALICE Collaboration on MWPC's which are intended to be used in a muon spectrometer at the LHC [48]. The chambers are remarkably similar to the ones used by MiniMax, and therefore the results obtained in the study may be relevant to the performance of the Min- 
iMax chambers. A pion beam was aimed at a lead absorber, and background particles from interactions in the lead were detected in a wire chamber located at the position which was predicted by simulations as the location of the shower maximum. A GEANT simulation proved to underestimate the measured number of charged particles into the chamber by a factor of 2-4. A simulation using stand-alone FLUKA (as opposed to the option of using FLUKA subroutines inside GEANT) produced a distribution of particles much closer to what was actually seen. Further analysis suggested that a significant contribution to the hits in the chambers was due to neutrons interacting in the mylar face of the chamber and knocking out protons which were then detected. Such interactions of low-energy neutrons are included in the stand-alone FLUKA. We have not been able to obtain the stand-alone FLUKA simulation code.

\subsubsection{GEANT trigger rates}

The trigger rates and cross sections for events in the GEANT simulation from the minimum-bias PYTHIA input are shown in Table 3.1. The total fraction of events which pass the trigger in GEANT is slightly more than $50 \%$, and is higher for lead-in runs than for lead-out by about $1 \%$ for all types of events. Single diffractive events in which the proton is dissociated are triggered on about $15 \%$ of the time, and about $10 \%$ of those with a fragmenting anti-proton pass the trigger. For double diffraction, the fraction 
is about $20 \%$.

Note that if we add the fraction of triggered single diffractive events (26\%) times the single diffractive cross section from CDF ( $9.46 \mathrm{mb})$ to the non-single diffractive fraction (79\%) times the corresponding CDF cross section (50.87 $\mathrm{mb}$ ), the result is that we should trigger on $42.7 \mathrm{mb}$, which agrees very well with the estimate in Sec. 2.2.3.

\subsection{DCC generator}

The operational definition of DCC used by the MiniMax collaboration is that DCC is a cluster of pions (domain) with neutral fraction $f$ given by the $1 /(2 \sqrt{f})$ distribution and with near-identical momenta which is nonrelativistic in the DCC rest frame. For the studies presented here, the domain size is taken to be on the order of the detector acceptance, and the momentum is such that the DCC is aimed at the center of the acceptance with a reasonably large $p_{T}$ in the lab frame.

We assume that the pions are non-relativistic in the cm frame of the DCC domain (and therefore refer to the domain as a "snowball"). We take the momentum distribution to be Gaussian with mean $\langle\vec{p}\rangle=0$ and variance $\langle\vec{p} \cdot \vec{p}\rangle=3 \sigma_{p}^{2}$. Since the DCC domain must be large enough to contain physical pions, i.e. have dimensions on the order of a few fm, uncertainty principle arguments require a small momentum, $\sigma_{p} \sim 50-100 \mathrm{MeV}$. We use the value $\sigma_{p}=100 \mathrm{MeV}$, which gives the pions a relatively large momentum 
in the lab frame, making them easier to detect. If the pions are not too relativistic in the $\mathrm{cm}$ frame, then the boosted DCC domain is approximately a circular disk of radius in lego space $R_{D C C} \sim \sigma_{p} / p_{T}$. For the MiniMax detector, with acceptance approximately a circle with radius 0.6 , a domain with $R_{D C C} \sim 0.7-1.0$ would be easiest to find, which implies $p_{T}=140 \mathrm{MeV}$. This size seems reasonable since the typical radius of a jet is 0.7 .

We also assume that the number of pions in the DCC domain is approximately independent of the pseudorapidity of the center of the boosted domain. A result of this is that the ratio $\psi$ of the mean energy density of a DCC pion to that of a generically-produced pion is approximately constant. Then the mean number of pions is given by

$$
\left\langle N_{\pi}\right\rangle \sim \psi \frac{p_{T}^{g e n}}{p_{T}}\left(\frac{d^{2} N}{d \eta d \phi}\right)_{g e n} \delta \eta \delta \phi
$$

or, substituting in the geometric values,

$$
\left\langle N_{\pi}\right\rangle \sim \psi \frac{p_{T}^{g e n}}{p_{T}} \frac{1}{2 \pi}\left(\frac{d N}{d \eta}\right)_{g e n} \pi R_{D C C}^{2}
$$

We take $(d N / d \eta)_{g e n}=6$ in the region of the acceptance, $p_{T}^{\text {gen }}=500 \mathrm{MeV}$, and $\psi=1$, which gives a mean number of pions $\left\langle N_{\pi}\right\rangle=5.0$. For a given event, the number of pions is taken from a Poisson distribution about this mean.

Next the charge of the pions is determined according to the $1 /(2 \sqrt{f})$ 
distribution. The neutral fraction in a given event is generated using the transformation method, where, if $x$ is a uniform deviate, then $f=x^{2}$ is distributed according to $1 /(2 \sqrt{f})$. A uniform deviate $y_{i}$ is then generated for each of the pions $\left(i=1, N_{\pi}\right)$; if $y_{i}<f$, the pion is defined to be neutral, otherwise it is defined to be charged.

Figures 3.13-3.15 show for the DCC pions: the momentum distributions, the distributions in $\eta$ and $\phi$ for a domain aimed at $\eta=4.1$ and $\phi=0.75$, the neutral-fraction distribution and the number distribution. Histograms of the number of events with given numbers of charged and neutral pions, for both total numbers and the numbers of those that enter the acceptance are shown in Fig. 3.16, and can be compared with that from generically-produced pions from PYTHIA in Fig. 3.17.

Unless noted otherwise, all further mention of the DCC generator refers to the use of the parameters given here. 


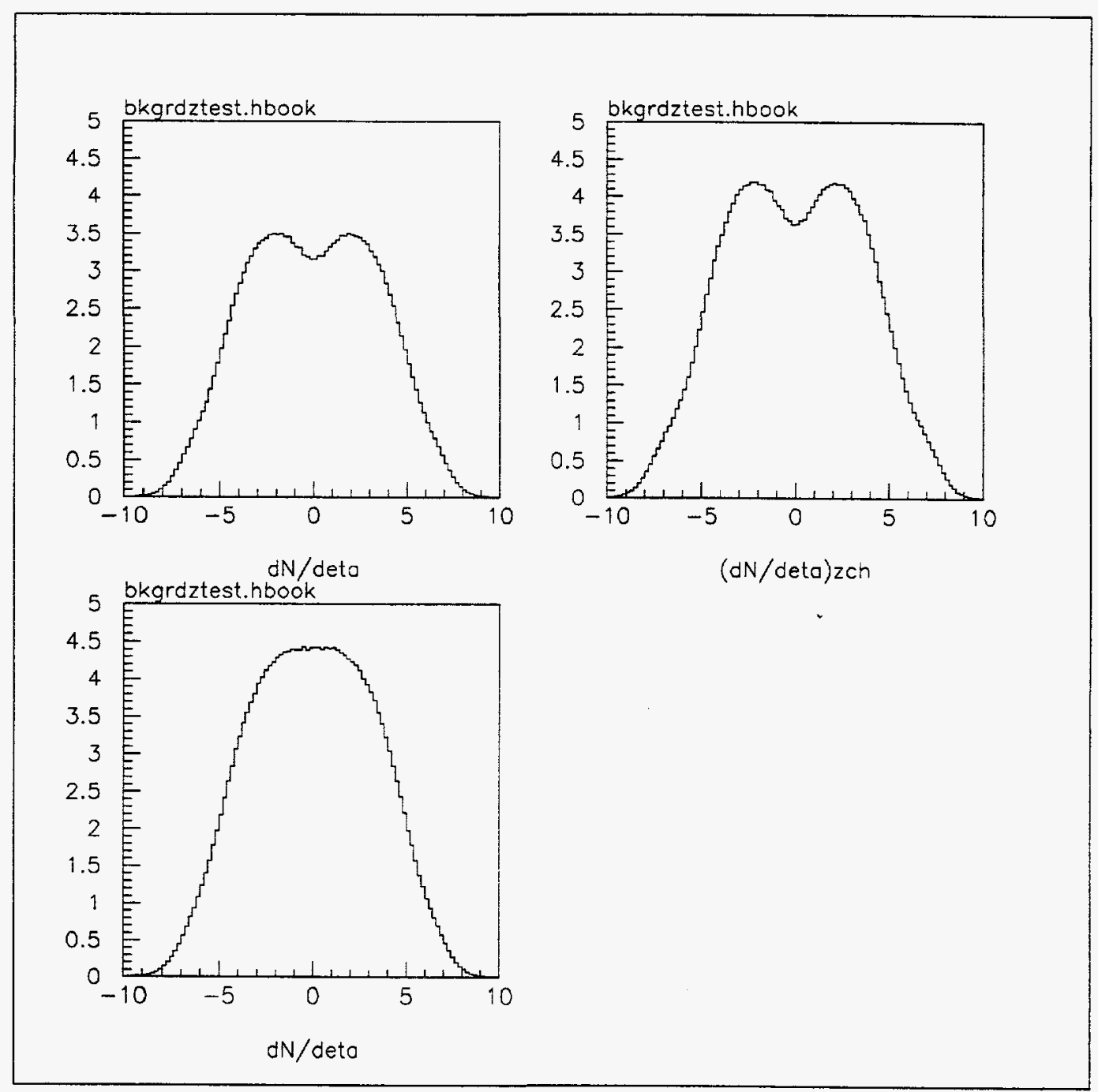

Figure 3.1: Pseudorapidity distribution $d N / d \eta$ for charged pions, all charged particles, and photons. 


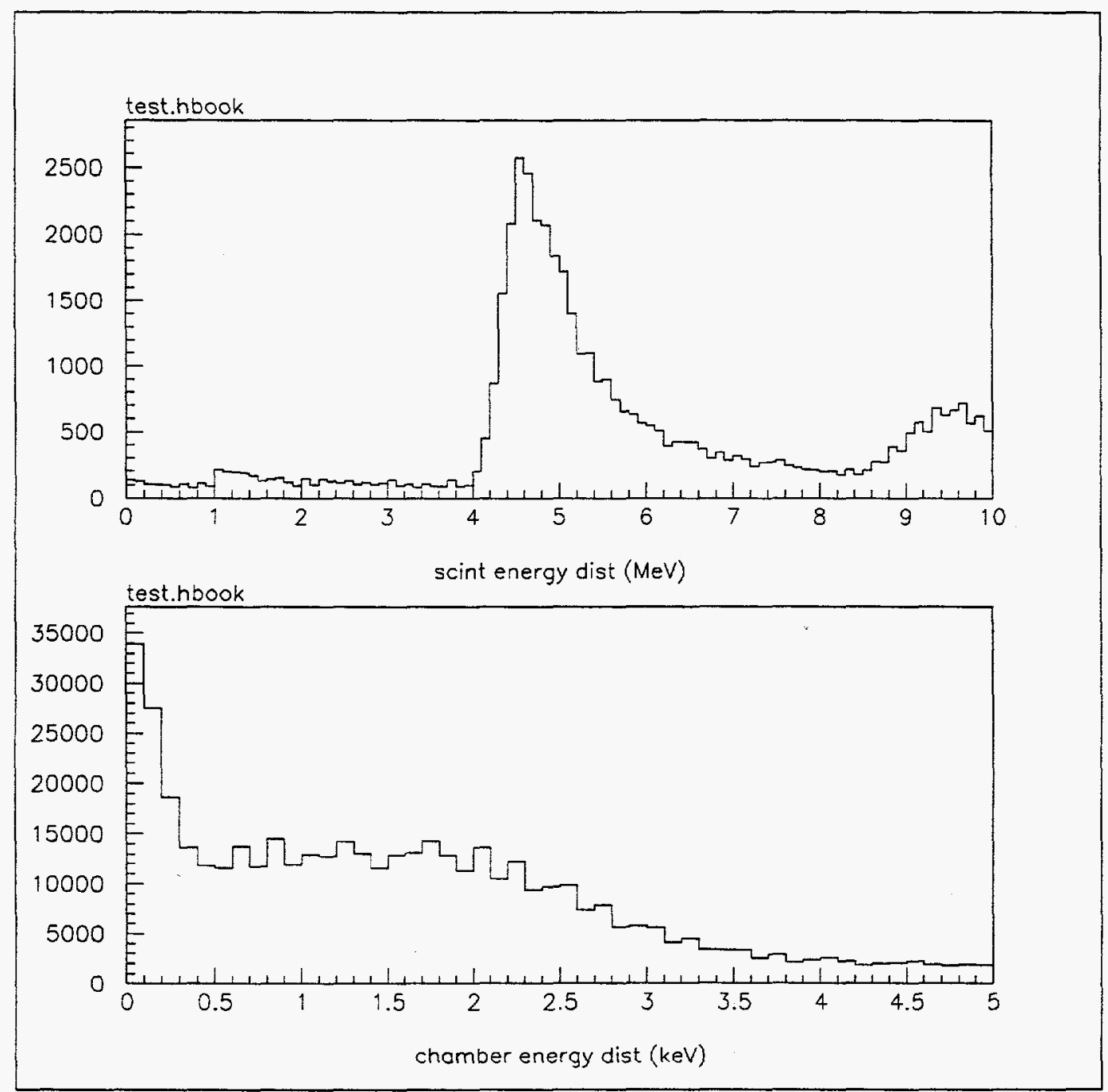

Figure 3.2: Energy deposited in (a) scintillator and (b) chambers in minimum bias events. 


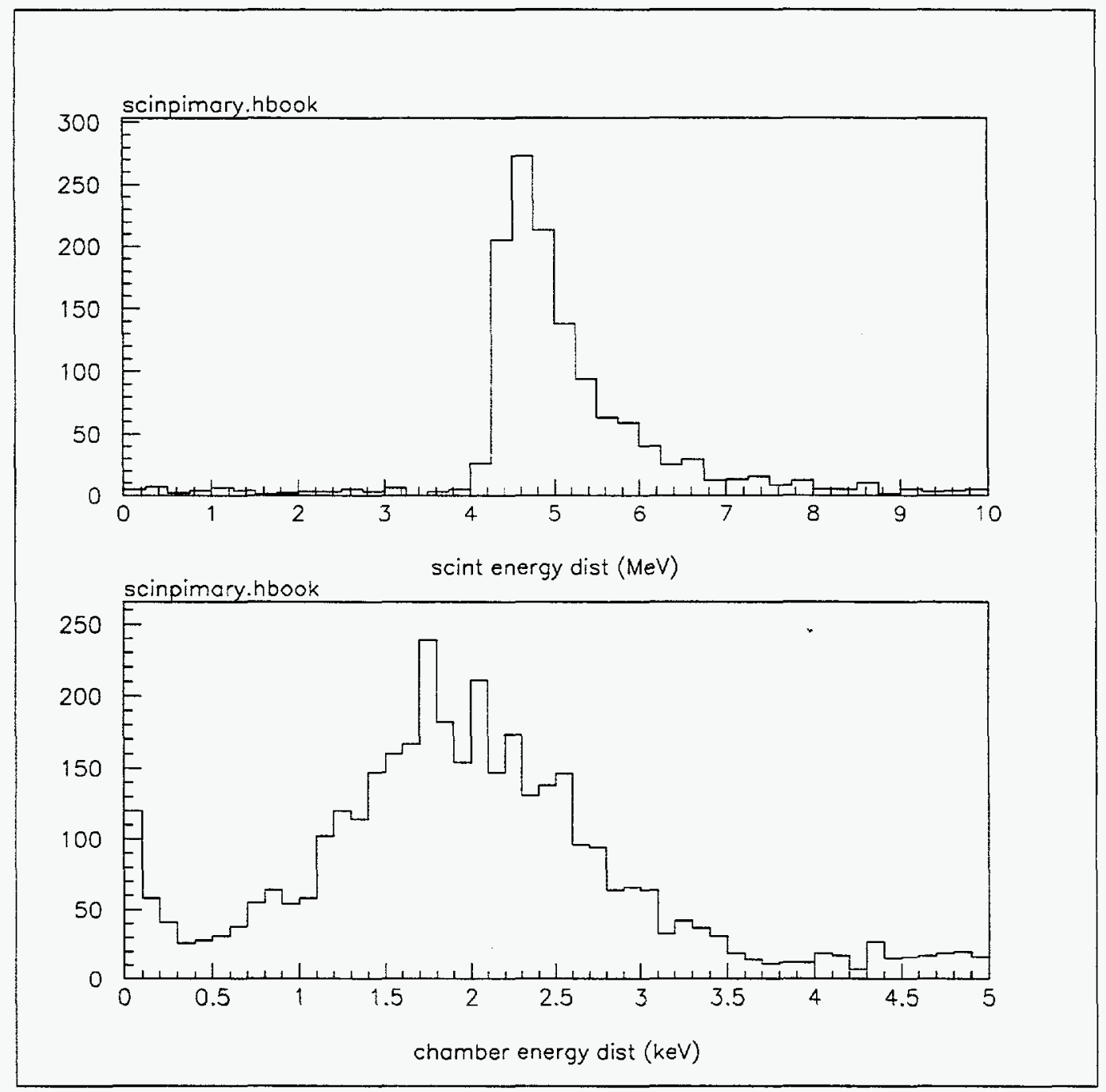

Figure 3.3: Energy deposited in (a) scintillator and (b) chambers by single charged pions. 


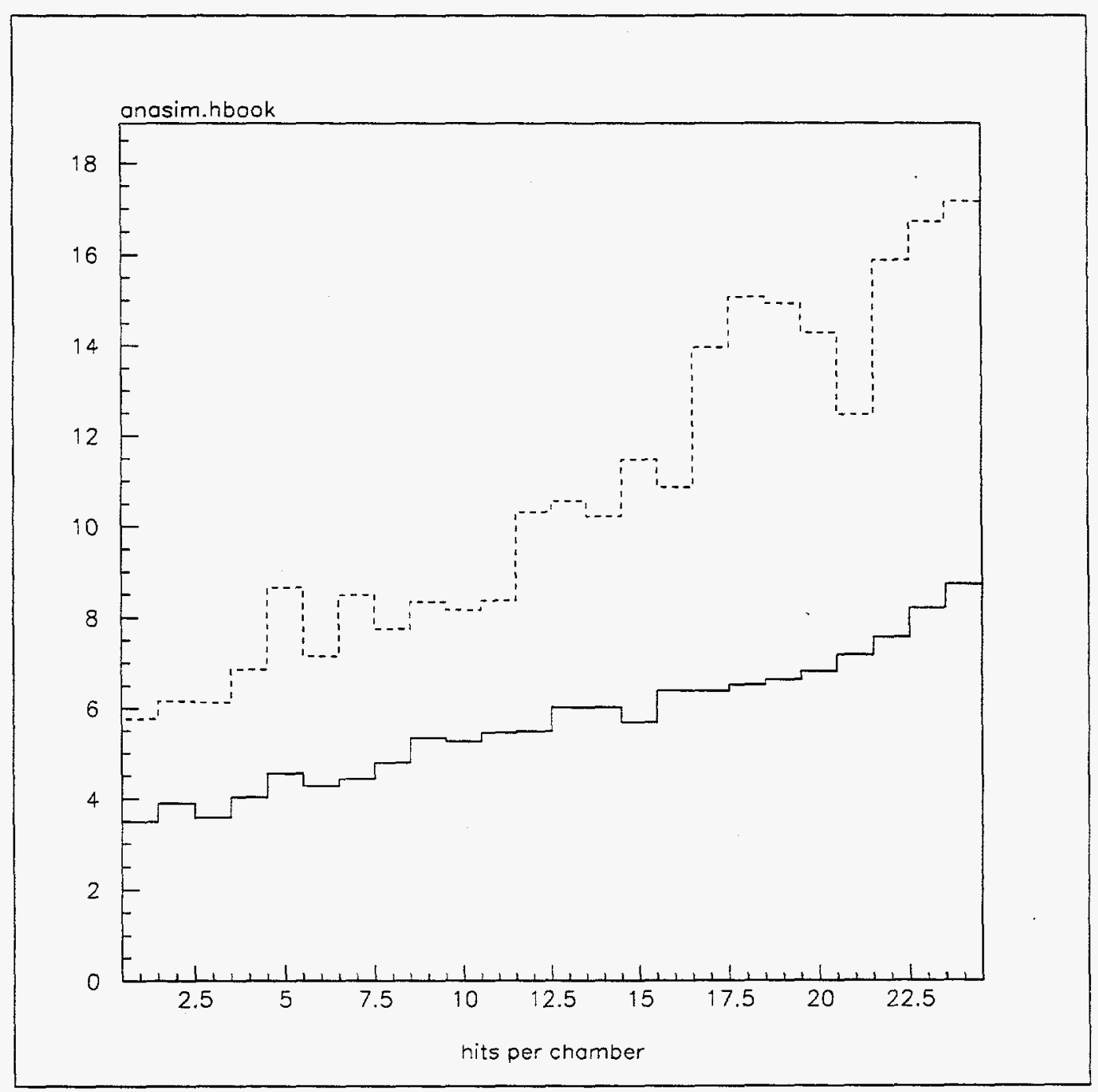

Figure 3.4: Mean number of wire hits per chamber: real (dashed) and GEANT with beam supports, etc (solid). 


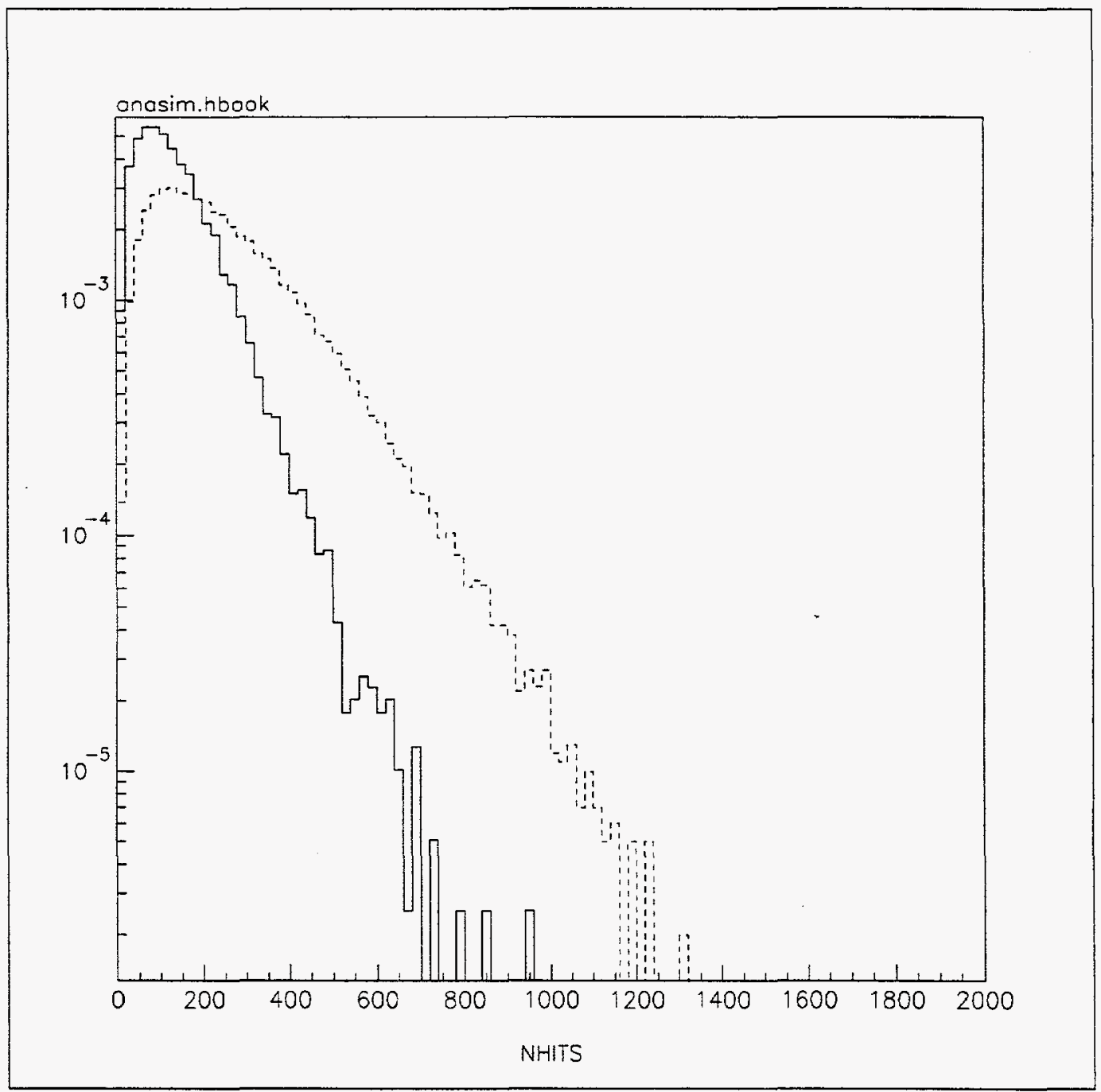

Figure 3.5: Distribution of NHITS: real (dashed) and GEANT with beam supports, etc (solid). 


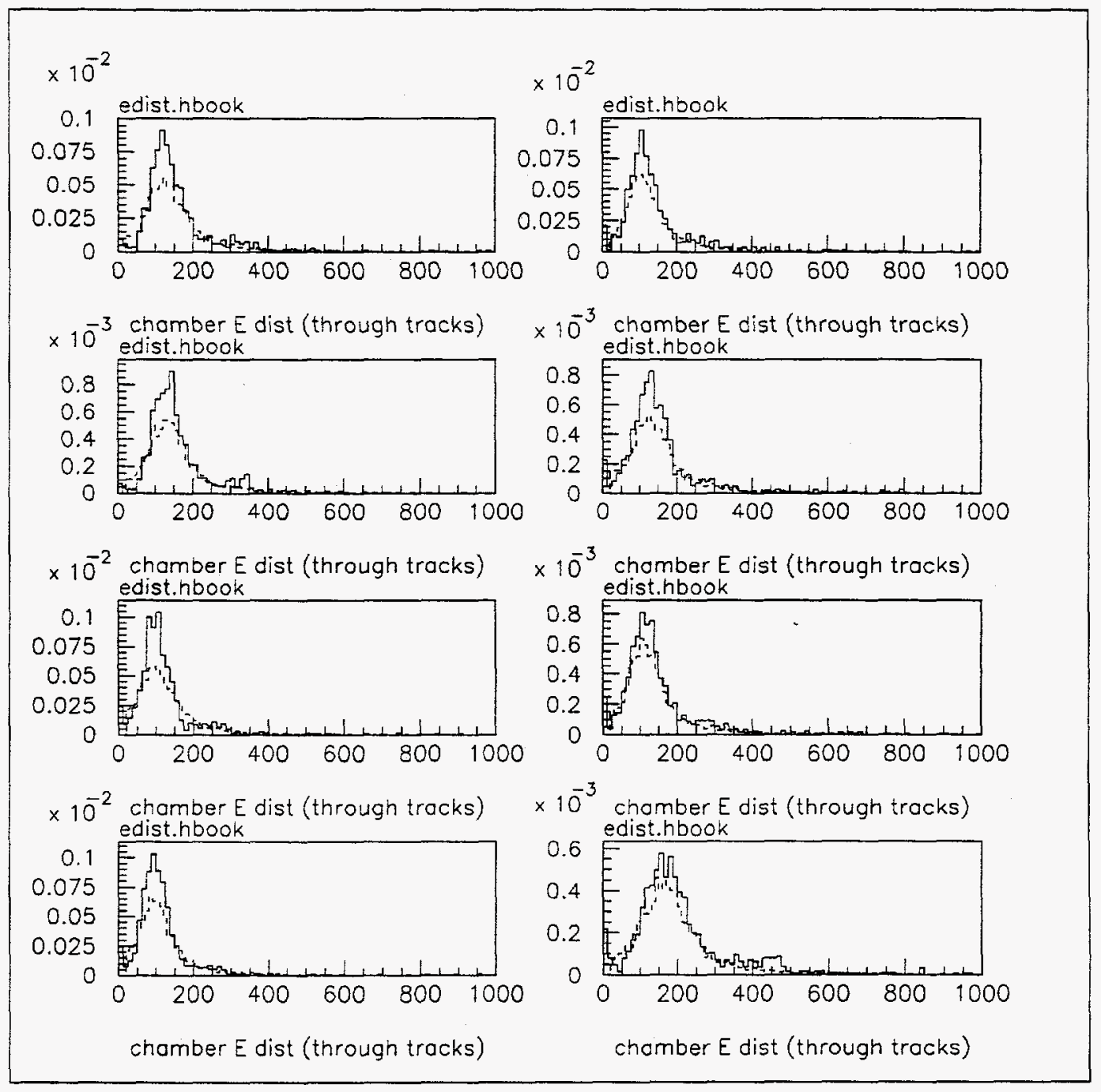

Figure 3.6: Pulse height distributions for chambers 1-4, 9-12, real (dashed) and GEANT (solid). 


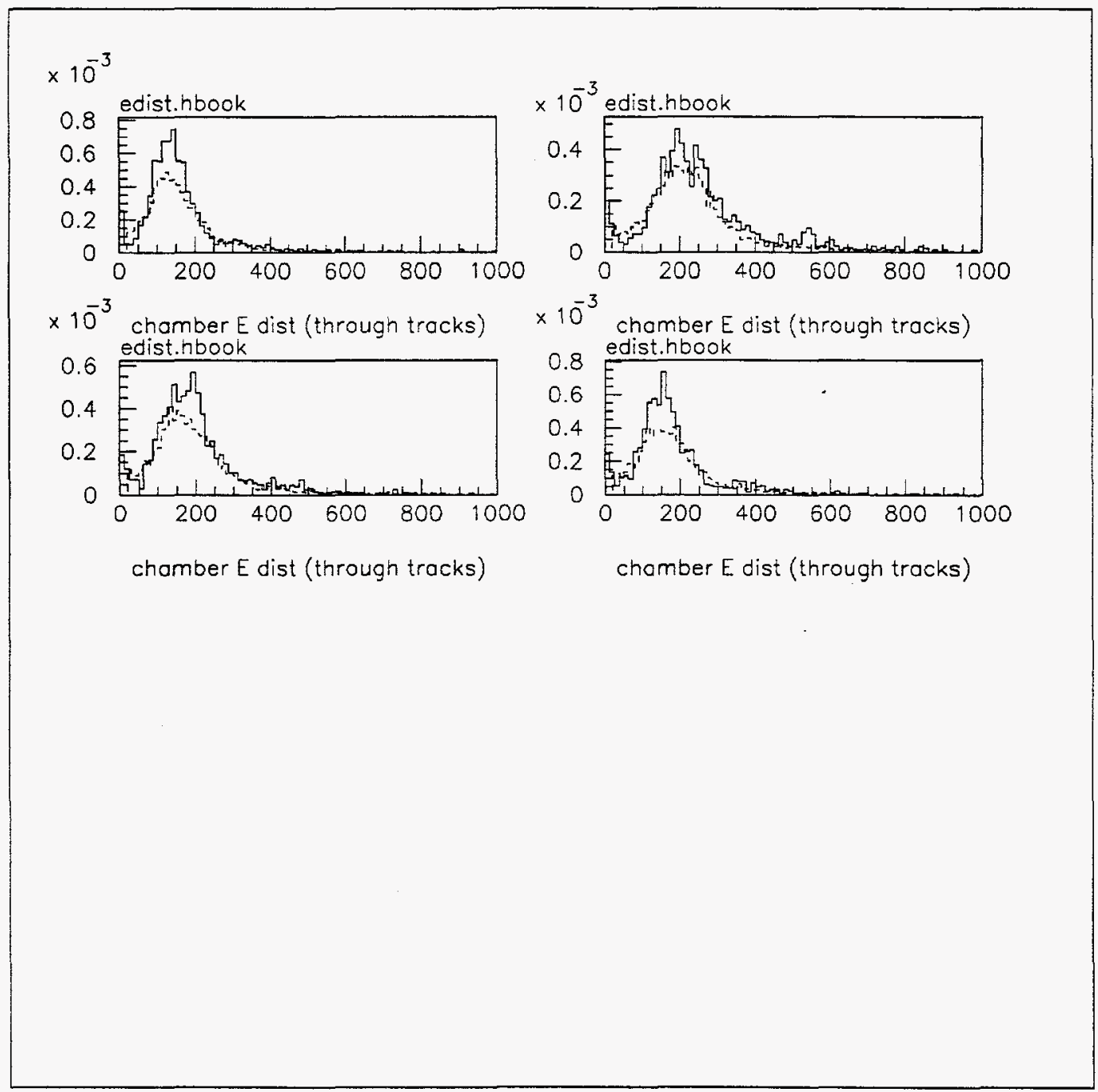

Figure 3.7: Pulse height distributions for chambers 21-24: real (dashed) and GEANT (solid). 


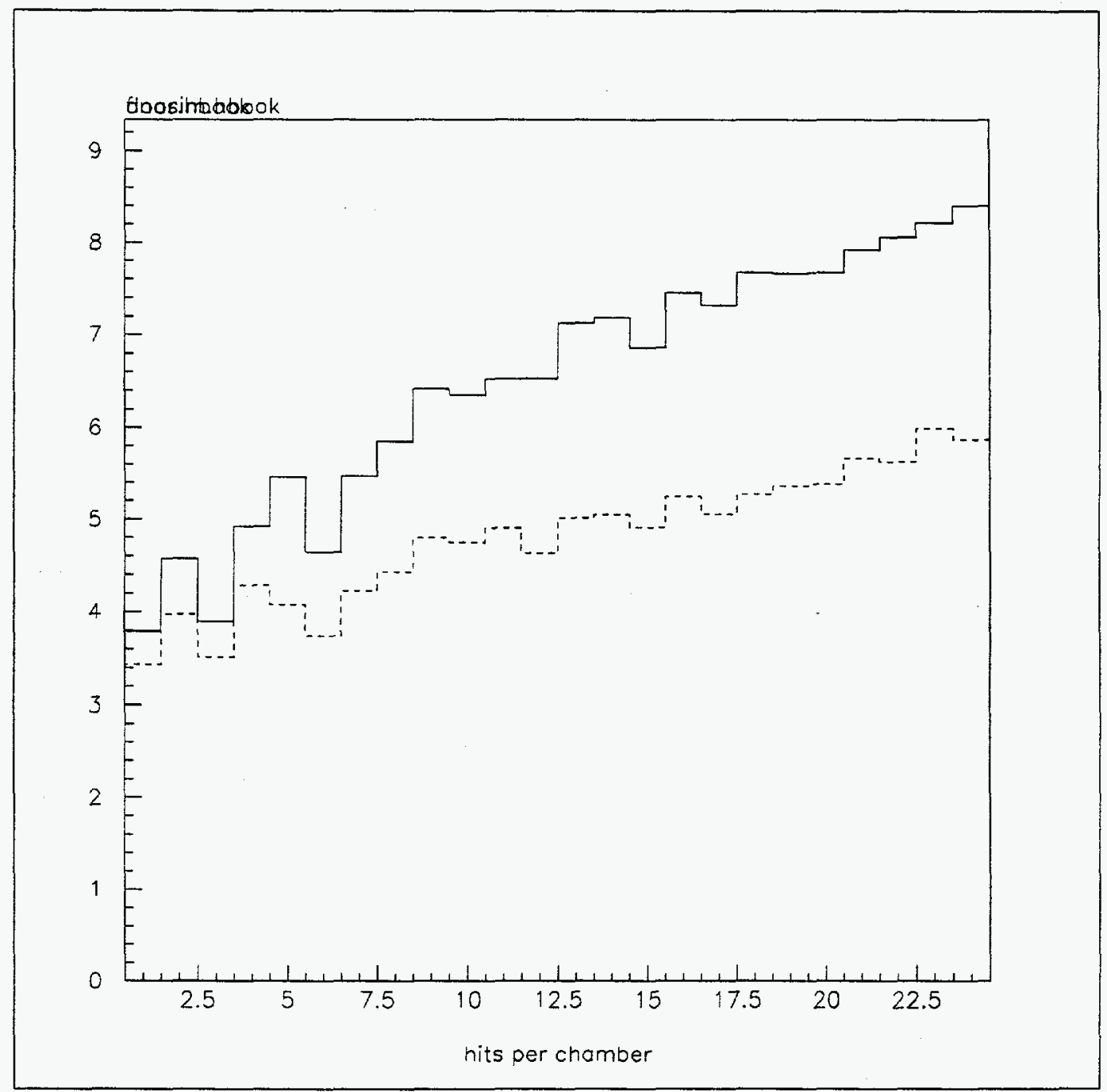

Figure 3.8: Mean number of wire hits in each chamber (solid) and with the beampipe removed (dashed). 


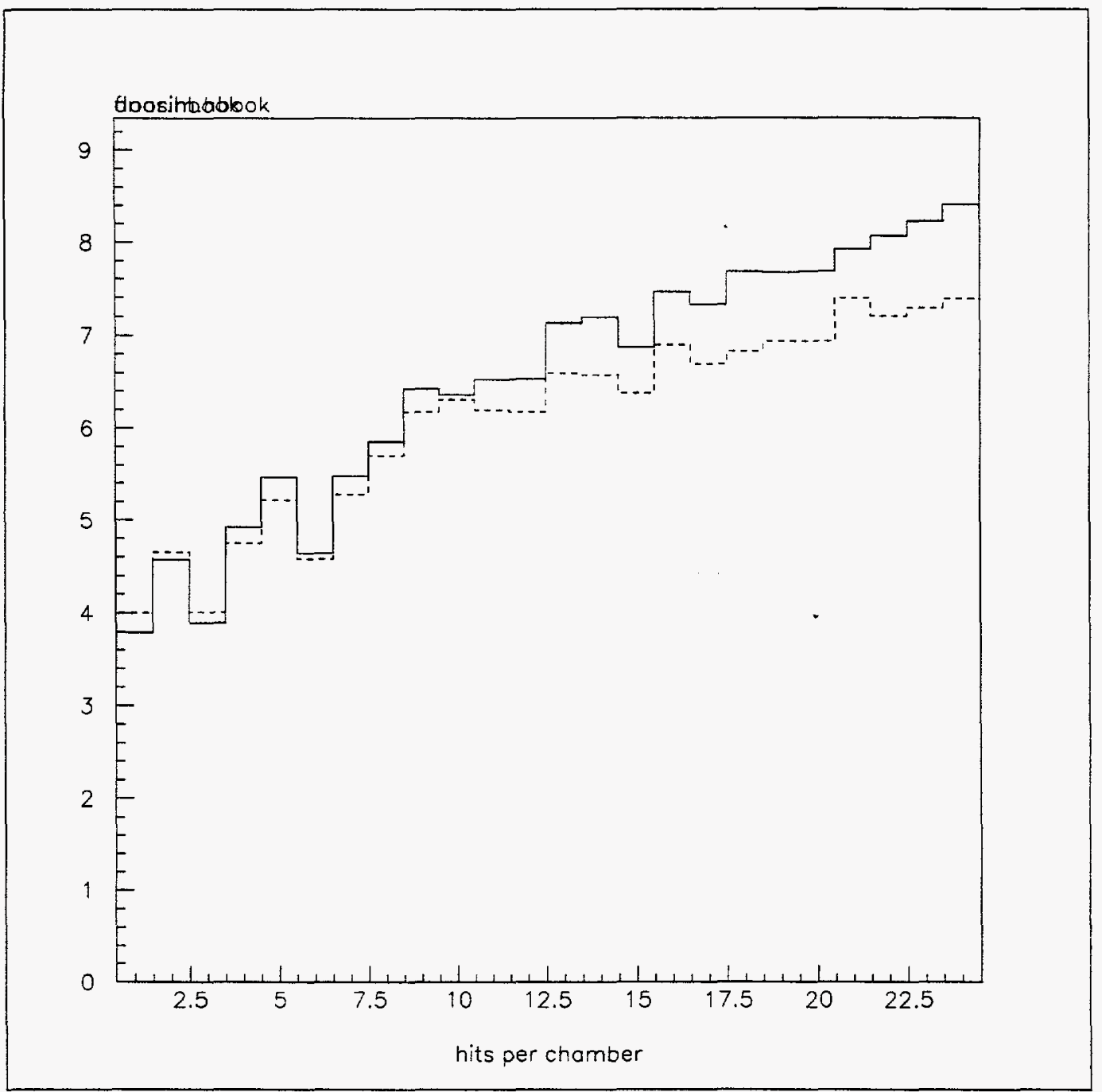

Figure 3.9: Mean number of wire hits in each chamber (solid) and with the abort pipe removed (dashed). 


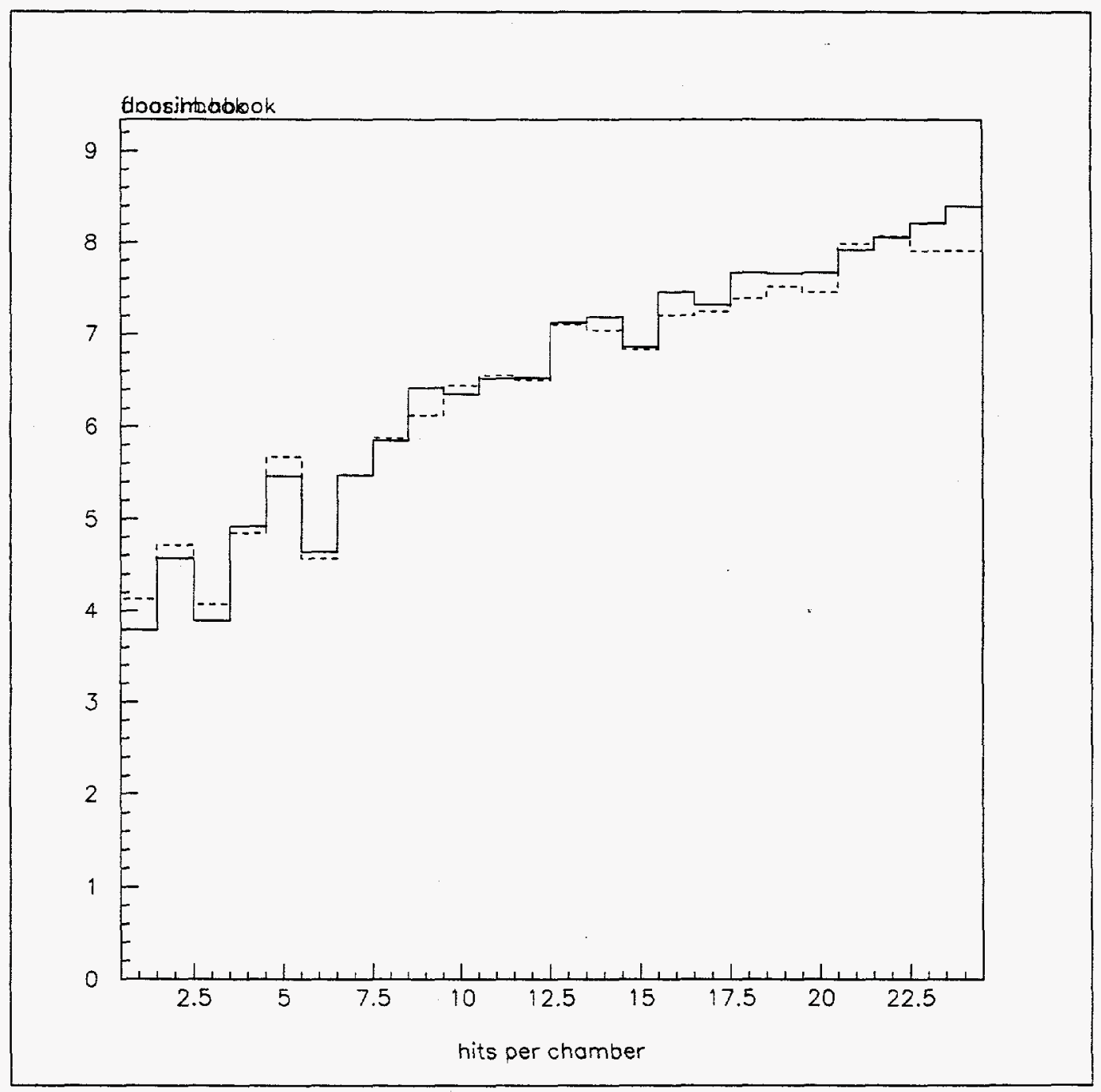

Figure 3.10: Mean number of wire hits in each chamber (solid) and with the chamber frames removed (dashed). 


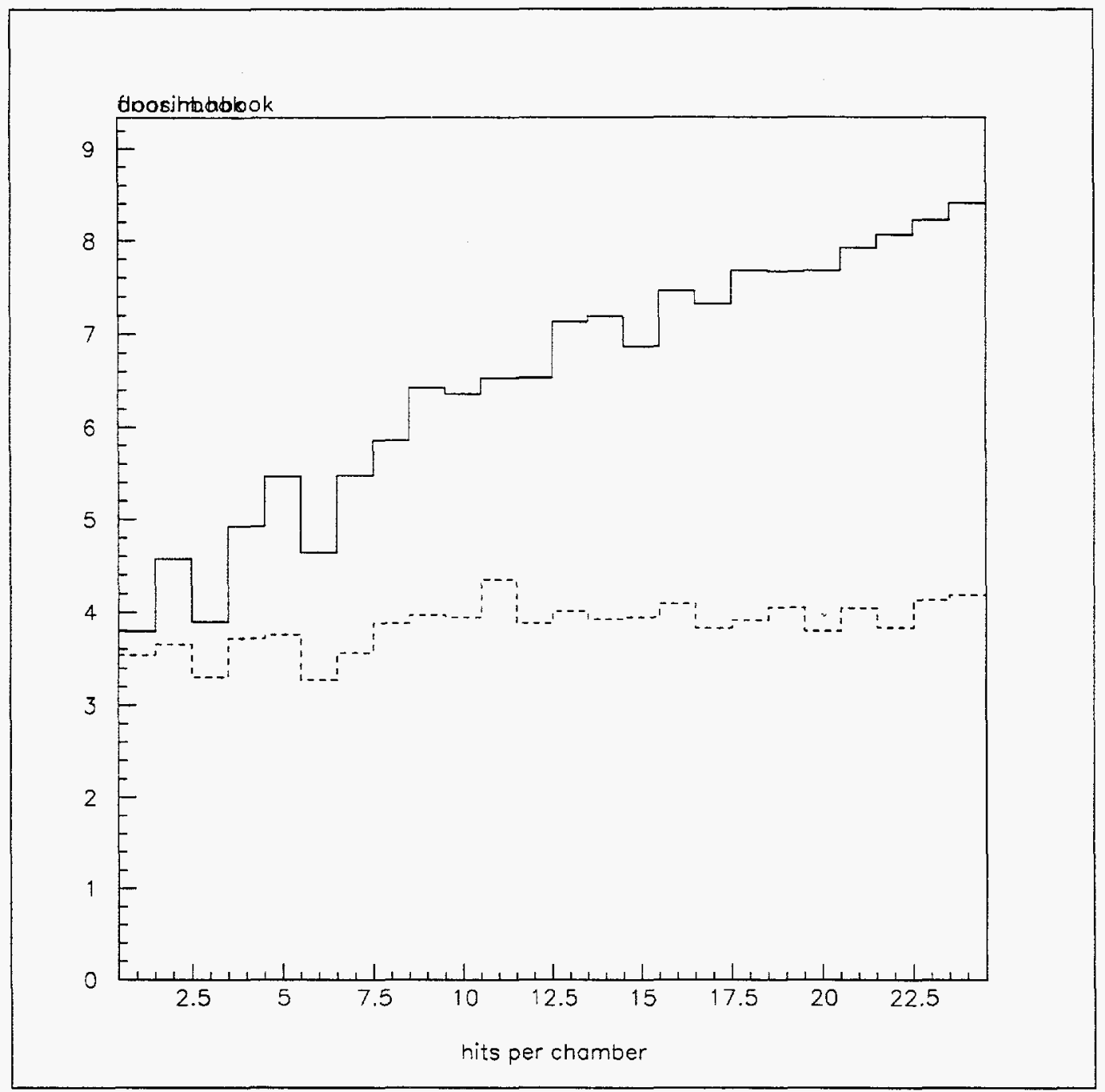

Figure 3.11: Mean number of wire hits in each chamber (solid) and with the beampipe, abort pipe, and chamber frames removed (dashed). 


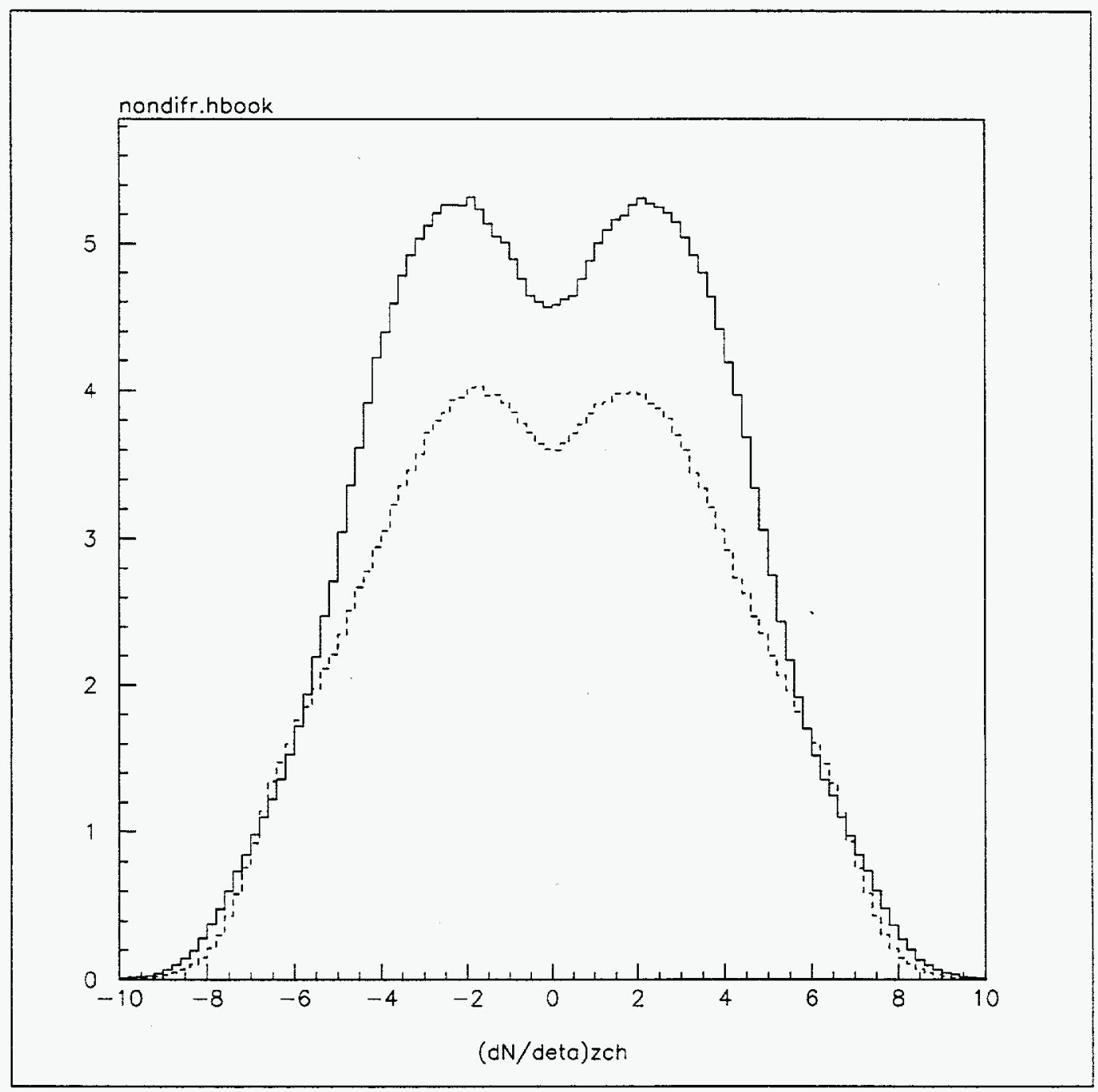

Figure 3.12: Charged-particle multiplicity $d N_{c h} / d \eta$ for non-diffractive PYTHIA (solid) and HERWIG (dashed). 


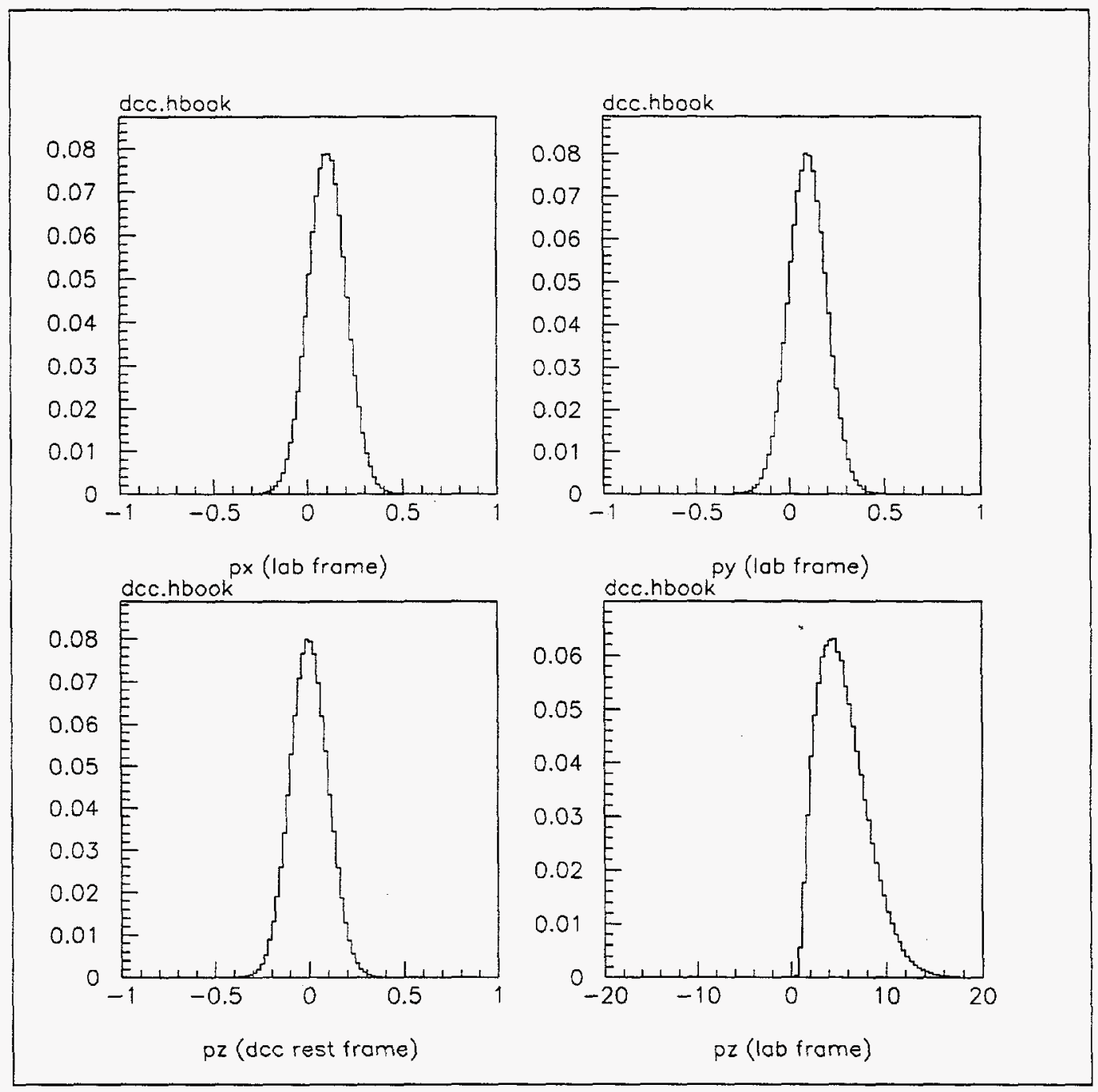

Figure 3.13: Momentum (GeV) of DCC pions: $p_{x}$ and $p_{y}$ in lab frame, $p_{z}$ in DCC rest frame, and $p_{z}$ in lab frame. 


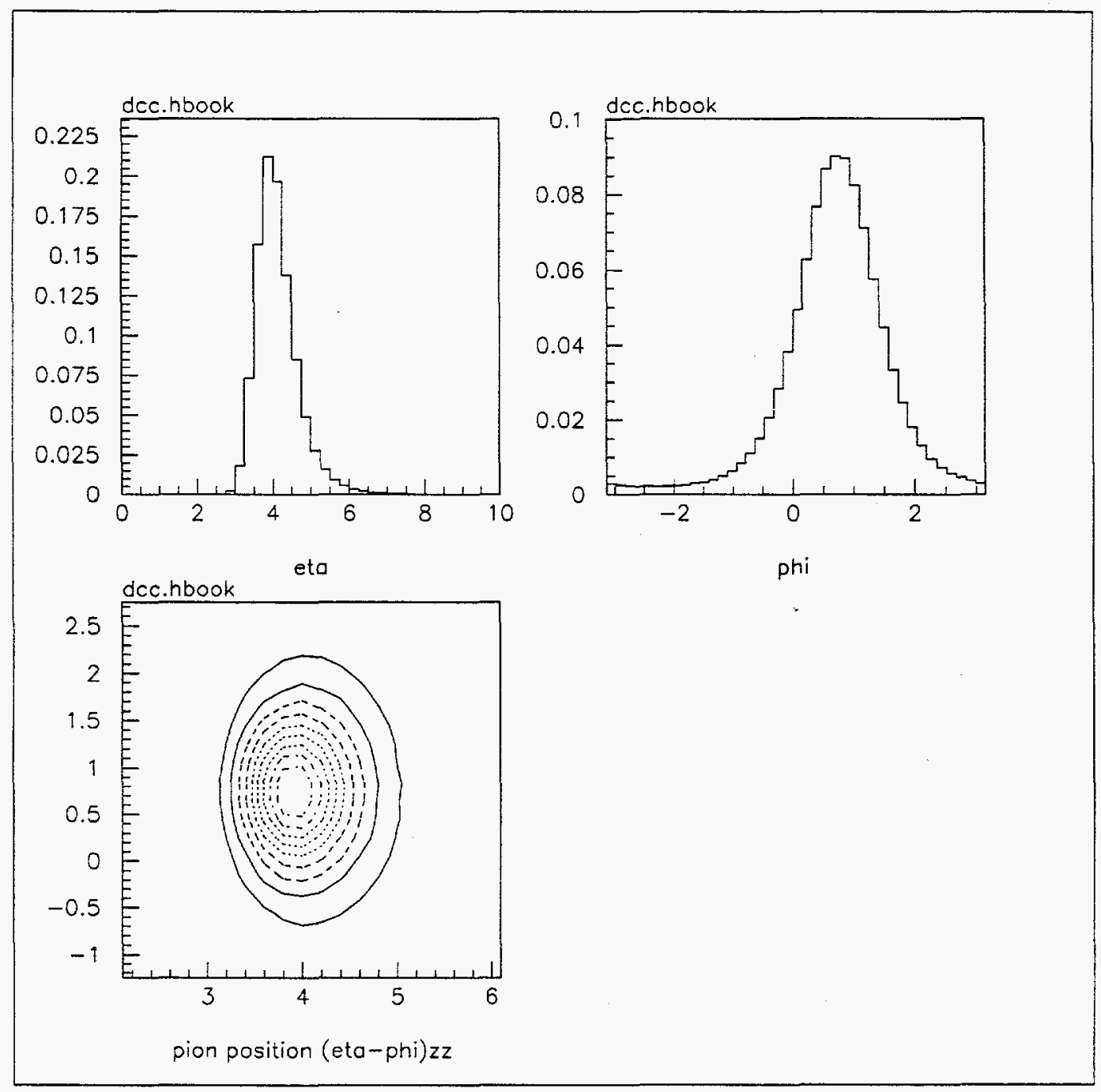

Figure 3.14: Location of the DCC pions in lego space. 


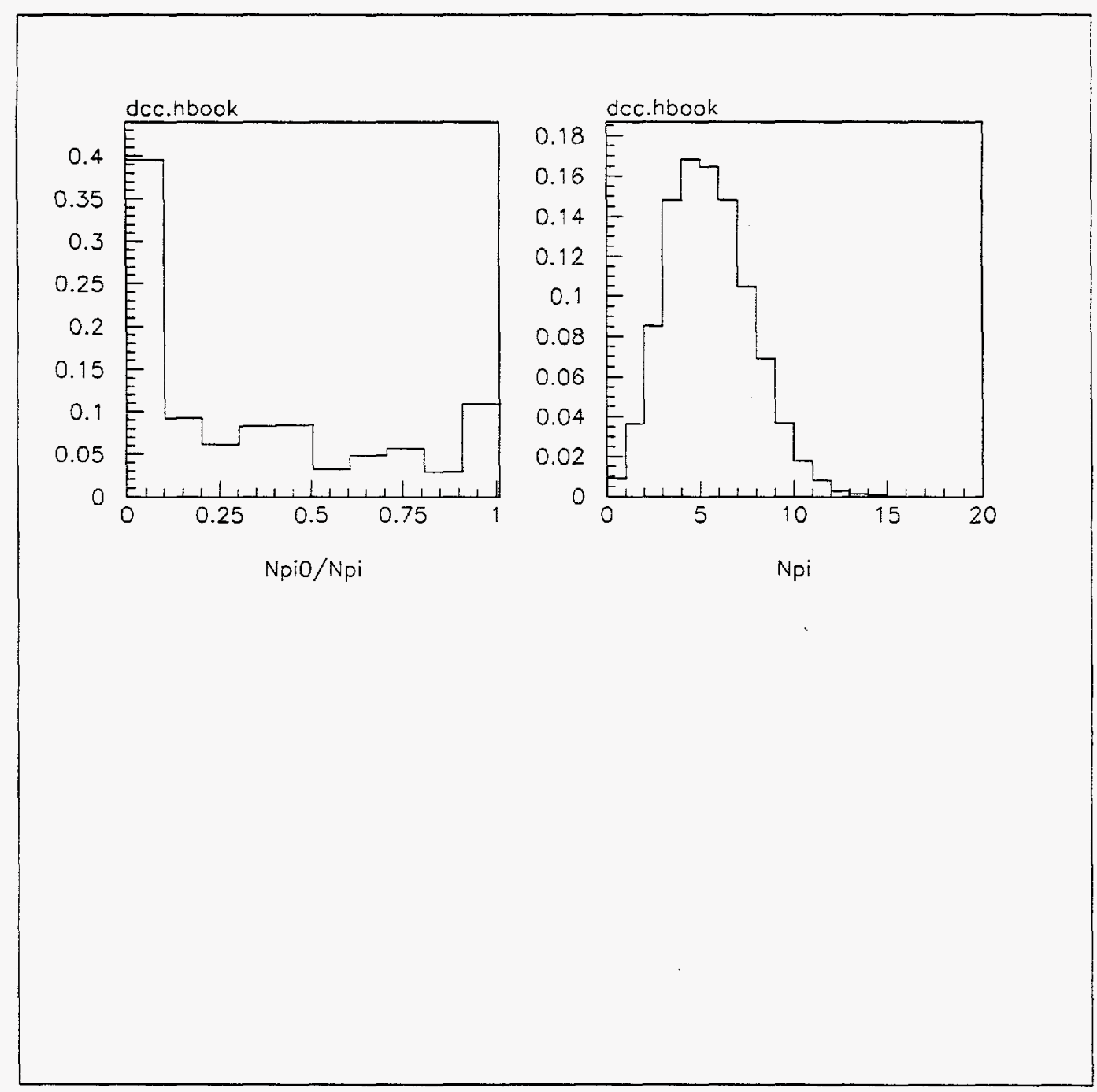

Figure 3.15: Distribution of fraction of DCC pions which are neutral $\left(N_{\pi^{0}} / N_{\pi}\right)$ and total number of DCC pions $\left(N_{\pi}\right)$. 


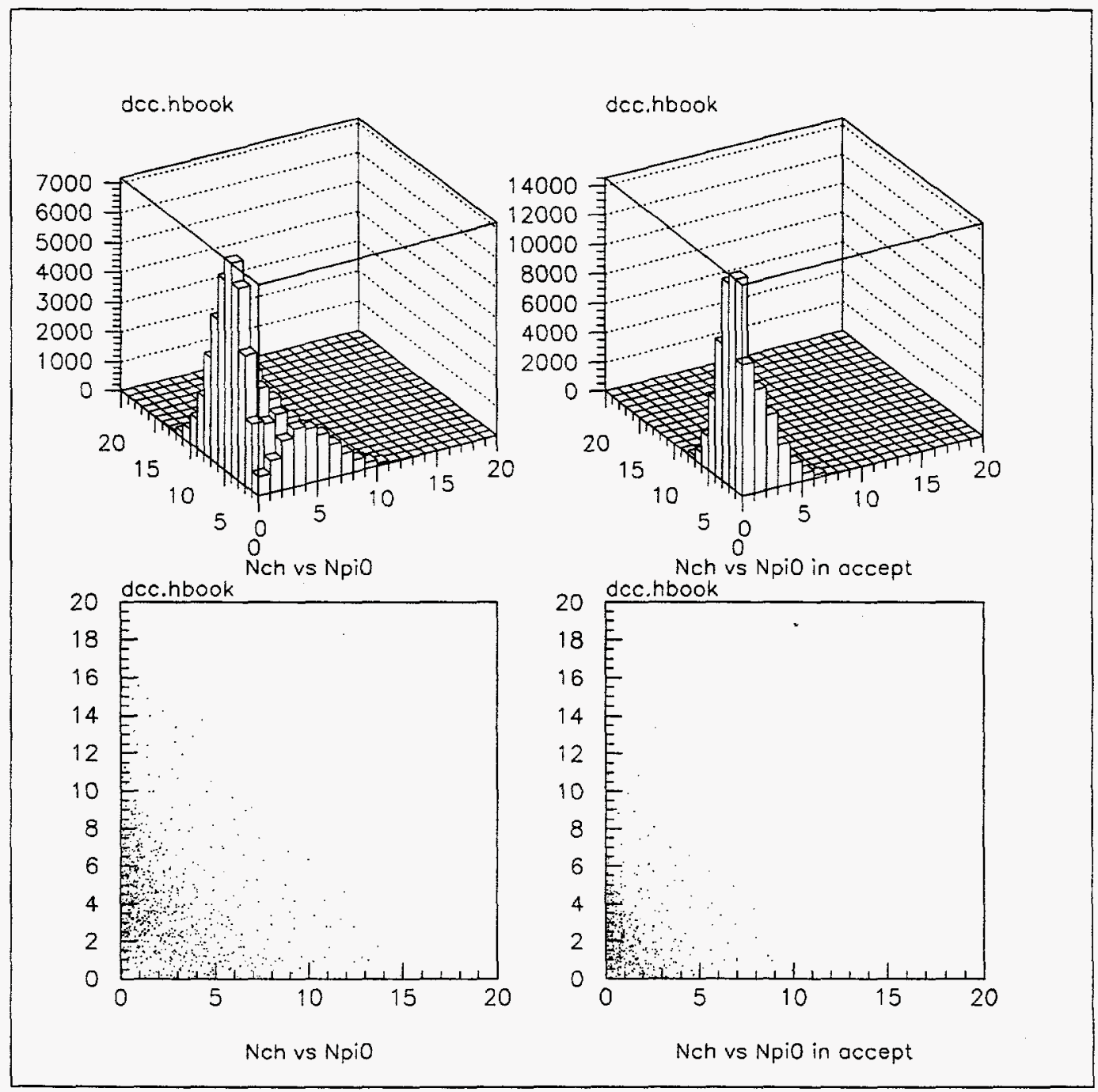

Figure 3.16: Number of charged vs number of neutral DCC pions: total number produced, and the number which enter the MiniMax acceptance. 


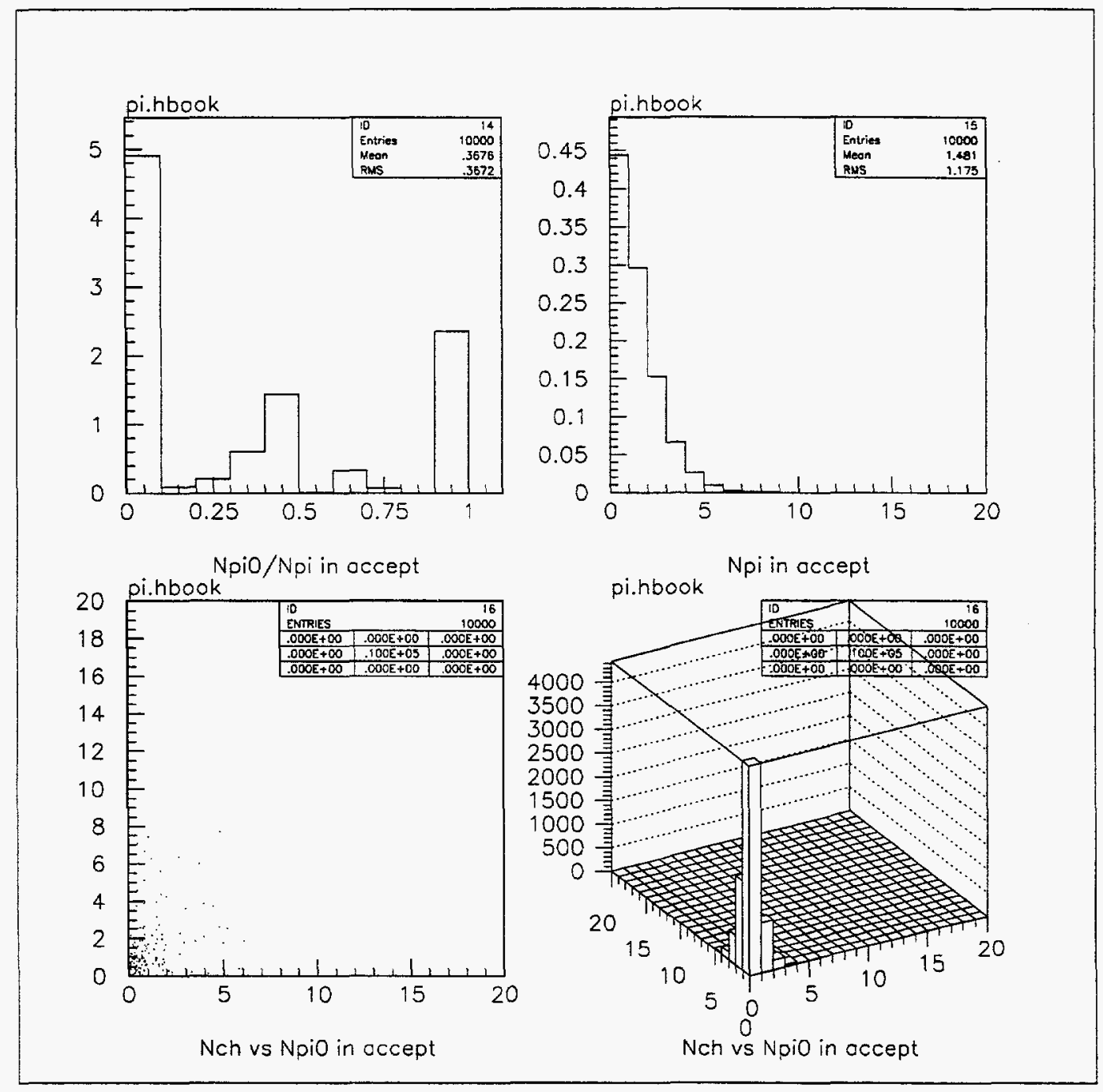

Figure 3.17: Number distributions of pions produced generically (by PYTHIA). 


\begin{tabular}{|l|c|r|r|r|r|r|}
\hline subprocess & $\begin{array}{l}\text { cross } \\
\text { section } \\
(\mathrm{mb})\end{array}$ & $\begin{array}{r}\text { number } \\
\text { of } \\
\text { events }\end{array}$ & $\begin{array}{r}\text { GEANT } \\
\text { trigger } \\
1 \mathrm{X}_{0}\end{array}$ & $\begin{array}{r}\text { GEANT } \\
\text { trigger } \\
\text { fraction }\end{array}$ & $\begin{array}{r}\text { GEANT } \\
\text { trigger } \\
0 \mathrm{X}_{0}\end{array}$ & $\begin{array}{r}\text { GEANT } \\
\text { trigger } \\
\text { fraction }\end{array}$ \\
\hline All included subprocesses & 72.98 & 300000 & 155709 & 0.5190 & 152069 & 0.5069 \\
$\mathrm{f}+\mathrm{f}^{\prime} \rightarrow \mathrm{f}+\mathrm{f}^{\prime}(\mathrm{QCD})$ & 2.217 & 9012 & 7494 & 0.8316 & 7289 & 0.8088 \\
$\mathrm{f}+\mathrm{f} \rightarrow \mathrm{f}^{\prime}+\mathrm{f}$ & 0.03198 & 130 & 98 & 0.7538 & 88 & 0.6769 \\
$\mathrm{f}+\mathrm{f} \rightarrow \mathrm{g}+\mathrm{g}$ & 0.03813 & 155 & 122 & 0.7871 & 121 & 0.7806 \\
$\mathrm{f}+\mathrm{g} \rightarrow \mathrm{f}+\mathrm{g}$ & 14.67 & 59642 & 52456 & 0.8795 & 51412 & 0.8620 \\
$\mathrm{~g}+\mathrm{g} \rightarrow \mathrm{f}+\mathrm{f}$ & 0.4667 & 1897 & 1719 & 0.9062 & 1692 & 0.8919 \\
$\mathrm{~g}+\mathrm{g} \rightarrow \mathrm{g}+\mathrm{g}$ & 21.42 & 87072 & 79857 & 0.9171 & 78618 & 0.9029 \\
Elastic scattering & 14.75 & 60825 & 0 & 0.0000 & 0 & 0.0000 \\
Single diffractive $(\mathrm{XB})$ & 6.194 & 25525 & 4057 & 0.1589 & 3815 & 0.1495 \\
Single diffractive (AX) & 6.194 & 25367 & 2706 & 0.1067 & 2452 & 0.0967 \\
Double diffractive & 6.839 & 28271 & 5920 & 0.2094 & 5336 & 0.1887 \\
Low-pT scattering & 0.1508 & 2104 & 1280 & 0.6084 & 1246 & 0.5922 \\
\hline
\end{tabular}

Table 3.1: PYTHIA cross sections and GEANT trigger rates 


\begin{tabular}{|c|c|c|c|c|c|c|c|c|c|c|c|}
\hline & \multicolumn{11}{|c|}{ pls hgt cut $/(0.4 \mathrm{keV})$} \\
\hline & 0.00 & 0.10 & 0.20 & 0.30 & 0.40 & 0.50 & 0.60 & 0.70 & 0.80 & 0.90 & 1.00 \\
\hline 0.0 & 65.24 & 63.58 & 62.11 & 60.82 & 59.50 & 58.39 & 57.45 & 56.58 & 55.79 & 55.16 & 54.46 \\
\hline 0.2 & 65.24 & 63.58 & 62.11 & 60.82 & 59.50 & 58.39 & 57.45 & 56.58 & 55.79 & 55.16 & 54.46 \\
\hline 0.4 & 65.24 & 63.58 & 62.11 & 60.82 & 59.50 & 58.39 & 57.45 & 56.58 & 55.79 & 55.16 & 54.46 \\
\hline 0.6 & 65.24 & 63.58 & 62.11 & 60.82 & 59.50 & 58.39 & 57.45 & 56.58 & 55.79 & 55.16 & 54.46 \\
\hline 0.8 & 65.24 & 63.58 & 62.11 & 60.82 & 59.50 & 58.39 & 57.45 & 56.58 & 55.79 & 55.16 & 54.46 \\
\hline \pm 1.0 & 65.24 & 63.58 & 62.11 & 60.82 & 59.50 & 58.39 & 57.45 & 56.58 & 55.79 & 55.16 & 54.46 \\
\hline 苞 1.2 & 71.32 & 69.50 & 67.88 & 66.47 & 65.00 & 63.78 & 62.74 & 61.77 & 60.91 & 60.21 & 59.45 \\
\hline 1.4 & 75.45 & 73.53 & 71.81 & 70.31 & 68.77 & 67.47 & 66.36 & 65.33 & 64.42 & 63.68 & 62.87 \\
\hline 壳 1.6 & 77.16 & 75.21 & 73.46 & 71.93 & 70.36 & 69.04 & 67.92 & 66.87 & 65.94 & 65.19 & 64.36 \\
\hline 1.8 & 78.35 & 76.39 & 74.61 & 73.05 & 71.47 & 70.13 & 68.99 & 67.93 & 66.99 & 66.22 & 65.39 \\
\hline 2.0 & 79.37 & 77.39 & 75.58 & 74.00 & 72.40 & 71.04 & 69.88 & 68.82 & 67.86 & 67.08 & 66.24 \\
\hline
\end{tabular}

Table 3.2: NHITS of "Michgan chambers" in GEANT (threshold $=20$ ) for varying pulse-height and scintillator cuts. 


\section{Chapter 4}

\section{Data Analysis Tools}

\subsection{Tracker}

At least three different track-finding programs were written and used by the MiniMax collaboration [49]. Each has a different algorithm for reconstructing tracks from hit wires, but all give similar results.

The work described here was done using the combinatorial tracker. That algorithm is constructed to find all possible combinations of the hit wires in four "crosshair chambers". At least three of these chambers have different orientations, so that a unique straight line can be drawn through any such combination of hits. The line is considered a potential track, and the noncrosshair chambers are searched for hit wires within 3.5 wire spacings of the line. If enough wire hits are found, a straight line is fit to the hits. Then, if the fitted track passes quality cuts such as cuts on the $\chi^{2}$ of the fit, the track 
is recorded. Several sets of crosshair chambers are used in order to increase the probability that a track and all the associated hit wires are found.

Tracks with a greater number of wires are always looked for first because they are more likely to be the correct track than a similar one with fewer wires; when two tracks are similar (have a large fraction of wires in common) the track found first has more weight in determining the track parameters. Also, the tracker code is written to search for tracks which are pointed such that they appear to originate near the collision point before allowing the tracks to point in any direction. Tracks are searched for in the following order. First the charged tracks that go straight through the detector are found and recorded. Then the tracking is run on the back 16 chambers and tracks which do not share too many wires (the exact numbers are given in Table 4.1) with previously-found charged tracks are recorded. These tracks include photon conversion tracks and segments of charged tracks which bend in the lead and are therefore not found as through-going charged. Tracks of the same type are then looked for in the eight chambers directly behind the lead (chambers 9-16) in case the tracks leave the acceptance before hitting enough chambers. Interactions in the beam pipe also produce background tracks which can be found in this region. Finally, the front eight chambers are searched for segments of charged tracks that are not found as going straight through all chambers. Figures 4.1-4.6 show event displays of various types of tracks. 
In the process of searching for each type of track, track candidates are compared to previously-found tracks to make sure that the same real track is not being recorded as many similar tracks. In the charged tracking, for example, tracks with hit wires in at least 22 chambers are searched for first. After a track is found, the wires hit by the track are stored in a list of used wires. As each new track candidate is found, its wires are checked against the used wires. If the candidate does not have at least 17 unique wires hit, it is dropped. This significantly reduces the number of fake tracks recorded by the tracker. The candidate track is also compared to the other tracks individually. The hits in each chamber are compared, and if the tracks are within two wires of each other in at least 16 chambers, the tracker determines that the same track has been found and the hits of the current candidate are added to the list of hits in the previously-found track. We refer to this as "grouping". Wires from the grouped track are added to the used-wire list. Next, tracks which go through only 21 chambers are considered. If such a track candidate has more than 17 wires which are in the used-wire list, the candidate is dropped, and if it has 16 wires within two wire spacings of hits in another track, the two tracks are grouped. Table 4.1 shows the various cuts on number of chambers hit or wires in common for each type of track.

The tracks are broken into segments in front of and behind the converter plane, referred to as "heads" and "tails", respectively. A dst is written, which, though traditionally stands for data summary tape, is just a file which 
contains, for each event, the event number, number of heads and number of tails, and then for each track segment, the number of wires hit followed by the list of wires. The wire number is given by (chamber -1$) \times 128+$ wire, for chambers 1-24 and wires 1-128 in each chamber. Earlier dst formats included the NHITS of the event (which can still be retrieved from the run data file) and the track parameters determined by a fit (which are now determined using separate code, as discussed in the next section). Sample dst entries are shown in Figs. 4.7, 4.8. The tracker is run once for each actual or simulated run, and the dst is used in all further analysis.

Only events with an NHITS less than 600 have been analyzed by the tracker. Higher-NHITS events tend to have large hit densities in the rear chambers, which leads to a huge number of potential tails, most of which are not real charged or photon-conversion tracks, and the tracker takes a longer time to analyze these events. About $6 \%$ of the events in lead-in runs have NHITS higher than this value. The NHITS is correlated with multiplicity, so that the sample of events which were analyzed by the tracker is somewhat biased towards lower-multiplicity events.

\subsection{Track fitter}

A separate program is necessary to fit tracks from the wires written in a dst since the fit parameters from the combinatorial tracker are calculated in the $(x, y)$ coordinate system instead of in $(u, v)$ (this is important because 
the resolution in $u$ is much better than in $v$ in the chambers behind the lead) and uncertainties in the track parameters are not reported. The code for the track fitter is given in Appendix B. With the track fitter, straight lines are fit to the hit wires in a track, parameterized by $u(z)=a_{u}+b_{u} z$ and $v(z)=a_{v}+b_{v} z$, and the covariance matrix (which gives the correlated uncertainties of parameters [50]) is generated. Uncertainties in the position of the track at a chamber are taken to be $1 / \sqrt{12}^{1}$ times the wire spacing for all hits.

Another subroutine (see Appendix B) is used to correct a small problem with tracks found by the tracker. Occasionally (in about $10 \%$ of the events), a tail is recorded which, in a single chamber, includes two wires as part of the same track which are separated by several wires; these double hits are present in most of the non- $u$ chambers of the track.

The cause turned out to be that two track candidates have common wires in all of the $u$-chambers, and enough close wires in other chambers for the tracker to group the track candidates into one track. In some cases, the candidates which are grouped in this way are two real tracks, but more often one of the candidates is a type of fake track which will be referred to as a "ghost". (A ghost is a track which borrows hits in the $u$ chambers from a single real track, and finishes up the track with random hits in the non- $u$

\footnotetext{
${ }^{1}$ For a hit to be recorded in a single wire in a chamber, a charged track must pass within 1 wire spacing of that wire. The uncertainty in position is then given by $(\Delta x)^{2}=$ $\left\langle x^{2}\right\rangle-\langle x\rangle^{2}=\int_{0}^{1} d x x^{2}-\left(\int_{0}^{1} d x\right)^{2}=1 / 12$, where $x$ is the distance in units of wire spacings.
} 
chambers such as from pipe-shower secondaries.) The track which is recorded is halfway between the two candidates.

Since the occurrence of these tracks with double hits is fairly rare, especially for those made of two real tracks, we do not try to separate out two distinct tracks, but rather to find one good track. The code used to correct this problem tries all possible combinations of hit wires such that the non- $u$ chambers each have only one wire included in the track. Only two of these combinations are considered as potential correct tracks: the one which best points (in $v$ ) towards the collision point, and the one which has the best $\chi^{2}$ when fit to a line in the $v-z$ plane. Of course, a single combination can satisfy both these requirements.

The pointing in $v$ is somewhat complicated by the fact that tracks going through the acceptance are at very small angles from the $v=0$ plane. Therefore, the uncertainty of the $z$ for which $v=0$ is much larger than that of $z(u=0)$. In other words, the uncertainty in the angle between the track and the $v=0$ plane and that angle are both comparably small. Instead, we use the following measure of pointing in $v$. We find the $z$ for which $u=0$ for the track, defined as $z_{0}$. Then, working in the $v-z$ plane, we define $\theta_{0}$ as the angle between the $z$-axis and a line drawn from $\left(v=0, z=z_{0}\right)$ to the point where the track being considered intersects the lead. The angle between the track itself and the $z$-axis in the $v-z$ plane is defined as $\theta$, and the uncertainty in that angle as $\sigma_{\theta}$. The measure of pointing is taken to be 
$\left|\theta_{0}-\theta\right| / \sigma_{\theta}$, which is small for tracks which point (to within uncertainty) to the same $z$ as is pointed to in $u$.

Figure 4.9 shows histograms of $\chi^{2}$ for tracks with the best $\chi^{2},\left|\theta_{0}-\theta\right| / \sigma_{\theta}$ for tracks with the best pointing, and then these values for good tails (those which did not have the double-hit problem). The plot of $\chi^{2}$ for good tails has an obvious separation of what we believe are real tracks and background, which we use to choose the cut $\chi^{2}<7$. For the pointing in $v$, we require $\left|\theta_{0}-\theta\right| / \sigma_{\theta}<8$. We are most interested in tails which point back to the collision point. (Further discussion of pointing cuts is given in Sec. 4.3.) Therefore, if the track with the best pointing also passes the $\chi^{2}$ cut, it is kept as a good track. If this is not the case, the track with the best $\chi^{2}$ is considered, and kept if it passes the pointing cut. In most cases, none of the combinations of wires produce a track which passes the pointing cuts, and the track is dropped.

\subsection{Vertexer}

Charged tracks from the collision point and photon conversion tracks are then reconstructed from the track segments. The vertexer code, given in Appendix $\mathrm{C}$, is used to determine the probability that track segments meet at a common point at the plane of the lead. A charged track is then defined as a head which either "matches" at least one tail or passes cuts discussed below, and a photon as any group of tails (or a single tail) not matched to 
anything in front of the lead. In terms of the notation (number of heads in match, number of tails in match), a charged track is a $(>0, \geq 0)$ and a photon is a $(0,>0)$. The location of the vertex is taken as the mean position of the included track segments at the lead, weighted by the uncertainties. In order to avoid effects from the edge of the lead, the vertex position is required to be within the region defined by 4.25 in $<u<10.25$ in, -3 in $<v<3$ in, and $\sqrt{(u-7.25 \text { in })^{2}+v^{2}}<4$ in, which is roughly the area 1 in from all sides of the lead.

The uncertainty in the position of the tail at the lead is apparently underestimated by propagating the uncertainties in track parameters calculated by the fitter. The most likely cause of this is multiple scattering in the lead. The mean variance in the $u$ position from this error propagation is $\sigma_{u}{ }^{2}=5.5 \times 10^{-4}$ in $^{2}$ and in $v$ is $\sigma_{v}{ }^{2}=0.030 \mathrm{in}^{2}$. A new estimate was found by histogramming the distance between two conversion tracks from a single photon in GEANT at the lead, and taking the standard deviation of the histogram as the uncertainty in position. Figure 4.10 shows these histograms, which give the new values $\sigma_{u}{ }^{2}=0.007$ in $^{2}$ and ${\sigma_{v}}^{2}=0.092 \mathrm{in}^{2}$. (The order of magnitude is really more important than the specific value, since cuts on other variables used in the vertexer can compensate for small differences in these values.) Calculated uncertainties for a track segment which are less than the new values are increased to the new values in the vertexer.

Tracks are required to point to within some distance of the mean collision 
point in order to remove background such as tracks from beampipe shower, and also combinatorial fakes. Figures 4.11 and 4.12 show the $z$ for which $u=0$ of heads and tails of $(1,1)$ charged tracks, and for single $(0,1)$ photon conversion tails. Heads of charged tracks are required to point to the region $-50<z<60$, which includes almost all primary charged tracks. Most of the tracks outside this region are fakes, although some are decay products of neutral particles such as the $\mathrm{K}_{s}^{0}$. Tails of charged tracks are not required to point so that tracks which multiple scatter in the lead will not be dropped. For lead-out runs (Fig. 4.11), most $(0,1)$ 's which point to $z \gtrsim 50$ are tracks from interactions in the beampipe, while for lead-in runs (Fig. 4.12), many are from photon conversions in the lead. In order to cut out the fakes from pipe shower, single photon conversion tracks are required to point to $-40<$ $z<50$. For photon conversions which produce more than one track, at least one track in the vertex must point to $z<50$. The pointing cut in $v$ is taken from the study in the previous section to be $\left|\left(\theta_{0}-\theta\right) / \sigma_{\theta}\right|<8$.

Parameters used in the vertexer code were determined by finding those which best reconstruct the PYTHIA and GEANT tracks, which are recorded in separate files. The pointing cuts, the increased uncertainties in the $u$ and $v$ position of tails, and cuts on the $\chi^{2}$ for the fit of how well two tracks match at the lead (which will be discussed in Sec. 4.3.1, see Fig. 4.13) are not independent; the cuts chosen are all self consistent. The code seems to work sufficiently well for both simulated and real data. As an example, Fig. 4.14 
shows the tracks which survived the vertexer cuts for the large NHITS event of Fig. 4.6.

Plots of efficiencies for finding charged tracks and photons are shown in Figs. 4.15-4.24. Efficiencies are given as a function of energy, transverse momentum, multiplicity, NHITS, and position in $\eta$ and $\phi$. The numbers of charged tracks and of photons in the acceptance as a function of these parameters are also shown, both because these distributions are interesting, and also to give an indication of the statistical significance of bins in the efficiency plots. The plots also include the mean number of fakes as a function of multiplicity and NHITS, and the mean number of actual charged tracks and photons as a function of NHITS.

\subsubsection{Matching tracks at the lead}

Tracks are "matched" or "vertexed" in the following way. Consider two tracks intersecting the lead at points $\xi_{1}=\left(\xi_{1}{ }^{1}, \xi_{1}{ }^{2}\right)=\left(u_{1}, v_{1}\right), \boldsymbol{\xi}_{2}=\left(\xi_{2}{ }^{1}, \xi_{2}{ }^{2}\right)=$ $\left(u_{2}, v_{2}\right)$, with covariance matrices $\mathbf{C}_{1}$ and $\mathbf{C}_{2}$. Assume that the two tracks meet at exactly the same point $\left[\bar{\xi}=\left(\bar{\xi}^{1}, \bar{\xi}^{2}\right)\right]$ at the lead. Also assume that the $\boldsymbol{\xi}_{\boldsymbol{i}}$ are Gaussian distributed about mean $\overline{\boldsymbol{\xi}}$.

The $\chi^{2}$ for the fit of the two tracks to $\bar{\xi}$ is

$$
\chi^{2}=\left(\xi_{1}-\bar{\xi}\right) \mathbf{C}_{1}^{-1}\left(\xi_{1}-\bar{\xi}\right)+\left(\xi_{2}-\bar{\xi}\right) \mathbf{C}_{2}^{-1}\left(\xi_{2}-\bar{\xi}\right)
$$


Minimizing $\chi^{2}$ with respect to $\bar{\xi}^{i}$ gives

$$
\bar{\xi}^{i}=\left[\left(\mathbf{C}_{1}{ }^{-1}+\mathbf{C}_{2}{ }^{-1}\right)^{-1}\right]^{i j}\left[\left(\mathbf{C}_{1}^{-1}\right)^{j k} \xi_{1}{ }^{k}+\left(\mathbf{C}_{2}{ }^{-1}\right)^{j k} \xi_{2}{ }^{k}\right] .
$$

Putting this $\bar{\xi}$ back into Eq. 4.1 yields $\chi^{2}$. There are four known parameters $\left(\xi_{1}\right.$ and $\left.\xi_{2}\right)$, and two which are determined $(\bar{\xi})$, leaving two degrees of freedom. The reduced $\chi^{2}$ is therefore $\chi^{2} / 2$.

All track segments in an event were matched together in order to find the cutoff in $\chi^{2} / 2$ for which matches with a lower $\chi^{2}$ are most likely real tracks, while those with a higher $\chi^{2}$ are most likely unrelated track segments. Plots of matches between pairs of heads, pairs of tails, and head-tail pairs are shown in Fig. 4.13. Head-head matches which have a low $\chi^{2}$ are due only to the heads being coincidentally close together at the lead. The peak at low $\chi^{2} / 2$ for head-tail matches is due to charged tracks, and is fairly cleanly separated from false matches. The tail-tail plot does not show a clear division, but a slight division is present at about the same value of $\chi^{2} / 2$ as the division for head-tail matches. (Note that the cutoff values could have been different for the head-tail and tail-tail matches since the uncertainties in position of the tails are much larger than those for heads.) A reasonable cut appears to be $\log \left(\chi^{2} / 2\right)<0.7$, or $\chi^{2} / 2<5$. 


\subsubsection{Tracks which may be grouped together}

The original vertexer algorithm grouped any track segments which matched any other track segments in a vertex. The uncertainty in position of the tails is large enough that this occasionally produced vertices which contained more than one real track. For instance, a $(1,1)$ charged track and a $(0, n)$ photon could be grouped into a $(1, n+1)$, or two charged tracks could become a $(2,2)$.

By demanding that all tails in a vertex match the head (which has a much smaller uncertainty), charged tracks are less likely to be grouped with other tracks. The vertexer code was therefore changed to look for charged tracks first and remove those tails which were matched to heads before matching tails together. The following is the result of this change for a sample of GEANT events.

Of the original $135(2,2)$ vertices, 104 have heads which do not match each other and were therefore separated. About $94 \%$ of these are really two charged tracks, and $6 \%$ are fake heads grouped with real tracks. The 31 vertices which have two heads that match each other consist of $48 \%$ fake heads along with other tracks, $13 \% \mathrm{e}^{+} \mathrm{e}^{-}$pairs from photon conversions in the window ("window conversions"), and $39 \%$ vertices with two charged tracks which are very close together at the lead.

The majority of $(1, n)$ 's which were separated into more than one vertex are charged tracks combined with tails from photons, fake tracks, or pipe shower tracks. The latter two types of tails are likely to be removed by 
pointing cuts when separated from the charged track. About $10 \%$ result from window conversions where a conversion secondary showers in the lead, and approximately $5-10 \%$ are single charged tracks with multiple tails (from interactions in the lead). Of those which remained $(1, n)$ 's, approximately $20 \%$ are pions which interact in the lead, $30 \%$ are pairs of a charged track and a fake tail, $25 \%$ result from window conversions, and $20 \%$ are pairs of a close charged track and a photon. The rest involve missing heads, decays in GEANT, etc. Only about half of the vertices which contained both a charged track and a photon were reclassified as such.

The overall improvement in vertexer performance due to these changes is small since these types of vertices are not very common. Efficiencies are improved for high multiplicity events, without introducing many fakes.

Studies were also done with the real data by adding a single charged track or photon conversion track from a clean event to every other event in the run. This was done at the level of the dst, so that the behavior of the tracker for the combined events is ignored. At this level, the efficiencies for finding all tracks of the individual events in the combined event are not compromised unless the added track is within some distance of a track in the other event. The mean distance between an added charged track and another charged track for a track to be missed is about $0.5 \mathrm{in}$, and for an added charged track and a photon conversion about 0.7 in. 


\subsubsection{Tail-less heads as charged tracks}

Often the tail of a low-energy charged track will not be detected due to multiple scattering in the lead which may cause it to stop or to bend out of the acceptance or to lose so much energy that it does not travel in a straight path through the rear chambers. In GEANT, most of the $(1,0)$ 's are real (collision-point) charged tracks which stopped in the lead or had very soft tails; some are from neutral particle decays. Those tail-less heads which are fakes are distinguished by the fact that they do not have hits in all eight of the front chambers. However, in the real data, the $(1,0)$ 's seem to include a large fraction of fakes. The ratio of the number of $(1,0)$ 's to the total number of identified charged tracks [including $(1,0)$ 's] is much higher for the real data (run 1125) than for GEANT, $17 \%$ vs $7 \%$, whereas if the energy distributions of charged tracks are similar for real data and GEANT, this fraction should be about the same. Eliminating those $(1,0)$ 's which do not have hits from all eight chambers reduces the fractions to $12 \%$ and $6 \%$. Other types of $(1,0)$ 's which appear to be fakes either share wires in all three $u$ or all three

$v$ chambers with another track (ghosts), or point to somewhere other than the intersection with the $z$-axis (in $u$ ) of other charged tracks in the event which go all the way through the detector.

Based on these observations, tight cuts are made which are intended to eliminate most fakes at the expense of losing some real charged $(1,0)$ 's. Any $(1,0)$ 's which share wires in all $u$ or all $v$ chambers are dropped. The pointing 
cut is defined by other charged tracks in the event; if there are none, it is not used. The collision point $z_{c p}$ is determined as the weighted mean of the point of intersection with the $z$-axis of any other heads of $(1,>0)$ charged tracks in the event, using the parameterization in $u$ of the heads and the associated uncertainties. Plots of the distance between the $z(u=0)$ of the $(1,0)$ and the mean $z_{c p}$ divided by $\sigma$, the root mean square of the uncertainty in these $z$, for both GEANT and real-data $(1,0)$ 's are used to choose the condition that the $z(u=0)$ of the $(1,0)$ must be within $2 \sigma$ of $z_{\text {cp }}$. This cuts out many real charged $(1,0)$ 's. These cuts reduce the ratio of "charged" $(1,0)$ 's to total charged tracks to $5.0 \%$ for GEANT and $9.6 \%$ for run 1125 . Although the fraction for the data is almost twice as large as that for the GEANT, no apparent qualities of the remaining $(1,0)$ 's suggest that they are fake.

\subsubsection{Middle-eight tracks}

Originally, tails which do not go through at least 14 of the rear chambers, but do go through the first eight chambers behind the lead were not used by the vertexer. The reason is that the rear chambers are often tlooded with pipe shower, which results in a large number of "middle-eight" tracks which are pipe shower tracks or combinatorial fakes which can be found as photon tracks by the vertexer. However, these tracks can be used carefully in certain circumstances. Allowing the middle-eight tracks to be vertexed as tails of charged tracks saves some real charged tracks that would otherwise be $(1,0)$ 's 
and might have be thrown away by the tight cuts. The vertexer also allows middle-eight tracks as photon conversions as long as they are vertexed with at least one other tail. This is important because the tight pointing cuts on $(0,1)$ photons are often failed by a single tail of a photon conversion which produces other tracks that leave the acceptance before reaching the final chambers. If a middle-eight tail is vertexed to such a tail then the vertex is not subject to the tight cuts. Also, since fake tracks are unlikely to vertex at the lead, a vertex made only of at least two middle-eight tracks is counted as a photon.

It turns out that very few events have middle-eight tracks which can be vertexed with other track segments at the lead, so that the effect on overall efficiencies is almost negligible. This may be related to the difficulty of getting a good fit for middle-eight tracks due to the lack of $v$-resolution. Fake charged tracks are occasionally created when a fake $(1,0)$ is coincidentally matched with a middle-eight tail. The middle-eight tracks were nevertheless included in the vertexing, since even a tiny improvement in the photonfinding efficiencies is welcome.

\subsubsection{Origin of fakes}

A charged track from the vertexer is classified as a fake if there are no charged tracks from PYTHIA inside the acceptance within the specified distances in $u$ and $v$ of 0.5 in and 1 in, respectively, of the identified track at the lead. A 
sample of events which have one fake charged track was taken from $1.5 \times 10^{5}$ GEANT events. In order to avoid effects due to the presence of photons, the events were required to have no photons observed or known to convert in the acceptance. The sample is divided into the following categories: events in which 0,1 , or 2 charged tracks are sent into the acceptance and one extra charged track is found (the total sample of events had $3402,1475,348$ events, respectively, in these classes, and 39,38 , and 38 events were used in this study), and those in which 0 charged tracks are sent into the acceptance and 2 are found (217 total events, 37 events used, accounting for 74 fake tracks). Of the 188 fake charged tracks,

150 are decay product(s) of $K_{s}$ 's or $\Lambda$ 's,

24 are secondary charged tracks from other decays or interactions in the material surrounding the detector

1 is from the conversion of a photon in the window,

12 are real charged tracks just outside the acceptance, and

1 is an actual combinatorial fake.

Decays of single $K_{s}$ 's or $\Lambda$ 's are responsible for about $70 \%$ of the events with two fake charged tracks. The important outcome of this study is that almost all of these "fakes" are caused by interesting physics processes or edge effects.

Also studied were 20 events (from a total of 416 ) where a photon conversion is present in GEANT ${ }^{2}$ and that photon is not found, and no PYTHIA

\footnotetext{
${ }^{2}$ GEANT conversions are defined as $\mathrm{e}^{ \pm}$tracks originating in the region containing the lead and scintillator between chambers 8 and 9 , and within 0.1 in at the lead of a photon
} 
charged tracks are sent into the acceptance, but one is found. In 11 of these events, the photon converts in the window, and a resulting $\mathrm{e}^{ \pm}$showers in the lead, i.e., the missed photon and fake charged track are correlated. The remaining events involve photons which are not found by the vertexer or are found just outside the acceptance, with uncorrelated fake charged tracks which fall into the categories mentioned above.

A photon found by the vertexer is defined as fake if no PYTHIA photon is aimed such that it will hit the lead within 0.5 in in $u$ and 1 in in $v$ of the identified conversion. In 38 events (from a total of 1800) where no charged tracks are sent into the acceptance by PYTHIA and no photon conversions from GEANT are present in the acceptance, and one photon is found,

19 are conversions of secondary photons, roughly half of which appear to be due to decays, and the other half due to interactions in the detector region,

14 are real photons just outside the acceptance, 2 are charged decay products of $K_{s}$ 's where the heads are lost, 1 is the product of an interaction of a neutron in the lead, and

2 are from events which are too complicated to determine the source of the fake.

Of 20 events (from a total of 319 ) where a charged particle is lost and a fake photon found, 17 involve a charged track which multiple scatters in the from PYTHIA. 
lead so that the tail is found as a photon conversion, but the head is dropped. In the remaining 3 events, the missed and fake tracks are uncorrelated.

When a pipe shower produces a large density of hits in the rear chambers, the probability is greatly increased for finding fake photons. Combinations of these hits, possibly together with hits from real tracks from the collision, form tracks which point to the collision region and therefore should be found by the tracker. Ghosts (described in Sec. 4.2) are a common example of such a fake track.

If pipe showers were uncorrelated with important observables such as the number of charged tracks and photons entering the acceptance in an event, then vetoing events which have pipe shower would reduce the number of fake photons found without biasing the remaining sample of events, say towards those with low multiplicity. Unfortunately, this is not the case. In order to study this, we first identified events with pipe-shower tracks. Primary tracks which are produced in the collision and pass all the way through the MWPC telescope occupy a very limited region of the phase space defined by the position and angle in $u$ at some $z$. (Recall that the acceptance is roughly defined by the coverage of the lead converter, which extends from $u=3.25$ in to 11.25 in and $v=-4.0$ in to 4.0 in at $z_{\text {lead }}=150$ in.) Figure 4.25 shows the location of tracks in $\left(u, \theta_{u}\right)$ space where $u=u\left(z_{\text {lead }}\right)$ and $\tan \theta_{u}=u\left(z_{\text {lead }}\right) / z_{\text {lead }}$, averaged over many events. The dense band near the center of the plot is due to primary charged tracks. We defined rough 
boundaries around this region and vetoed events with any tails in the regions $\theta_{u}>0.009 u+0.016$ or $\theta_{u}<0.006 u-0.028$, excluding tails which the vertexer found as photon conversion tracks. (Note that this is a very tight cut.)

Because of the higher hit density in the rear chambers created by pipe shower, the events which remain after the pipe-shower veto have a much lower mean NHITS than that for all events. In GEANT, the mean NHITS for lead-in runs dropped from 142 to 90, and in run 1125 (lead in) from 229 to 113. Also, surviving events with higher NHITS tend to have more real tracks, as is shown for GEANT in Fig. 4.26. This figure also shows the distributions of actual charged-track and photon multiplicities (from PYTHIA), which appear to be biased towards lower multiplicities. This seems to be true to a greater extent for the real data; Figure 4.27 shows that the probability distribution of the observed number of photons before and after the pipeshower veto for real data is more biased towards lower multiplicities than that for GEANT. The mean number of charged tracks found decreased from $\left\langle n_{c h}\right\rangle=0.53$ for all GEANT events to 0.47 for this sample, and of photons from $\left\langle n_{\gamma}\right\rangle=0.21$ to 0.14 . For the data (run 1125), the cut is even harder: $\left\langle n_{c h}\right\rangle=0.48$ falls to 0.32 and $\left\langle n_{\gamma}\right\rangle=0.19$ drops to 0.06 , practically no photons. We expect the veto to have a greater effect on the data because we believe that the real events contain more pipe shower than the GEANT, but since the vetoed events are apparently correlated with high multiplicity events, the remaining events are a clean, but not unbiased, sample. 
This sample of non-pipe-shower events is useful for studying fake photons which were not caused by pipe shower, although the events are also less likely to contain converted photons. Fake photons in two sets of the remaining events were classified. The first set was taken from about $10^{4}$ minimum-bias (triggered) events, and contains 104 events which pass the pipe-shower veto and have a fake photon. The second contains 71 such events which also have at least four primary charged particles and/or photons (from PYTHIA) in the acceptance; this sample includes all events of that type in the approximately $1.5 \times 10^{5}$ triggered events.

Of the fakes in the first set, 5 are ghosts,

11 are tails of charged tracks which are not vertexed with the heads, 9 are associated with photon conversions but are not vertexed with the other conversion tracks, 31 are other secondary photons such as those produced by interactions in the beampipe,

2 are secondaries from the interactions of neutrons in the lead,

11 are photons from the decays of $\pi^{0}$ 's produced by $\mathrm{K}_{s} \rightarrow \pi^{0} \pi^{0}$,

18 are real photons just outside the acceptance, and the remaining 17 either are not able to be classified, or fell into unique categories. The first few types of fakes are the most serious because they are correlated with the presence of real tracks. In almost all cases, when the 
tail of a charged track is not vertexed to the head, the tail-less head does not pass the cuts necessary to be counted as a charged track. Therefore a charged track is lost and is replaced by a fake photon. (This complicates the generating function analysis described in Chapter 5 , because it introduces a correlation between charged-track and photon efficiencies.) The classification of photons which are outside the acceptance but are found inside the acceptance as fakes is really just due to details in the code for finding fakes.

Due to the higher multiplicities in the second set, we expect more fakes associated with real tracks, such as ghosts. We find that this is true, but not to an extent which would seriously invalidate the assumptions made in the next chapter. The fake photons include

8 ghosts,

25 tails of charged tracks,

12 tracks associated with a photon conversion,

11 other secondary photons,

2 photons from $\mathrm{K}_{s} \rightarrow \pi^{0} \pi^{0}$,

9 photons just outside the acceptance, and 4 unclassified fakes. 


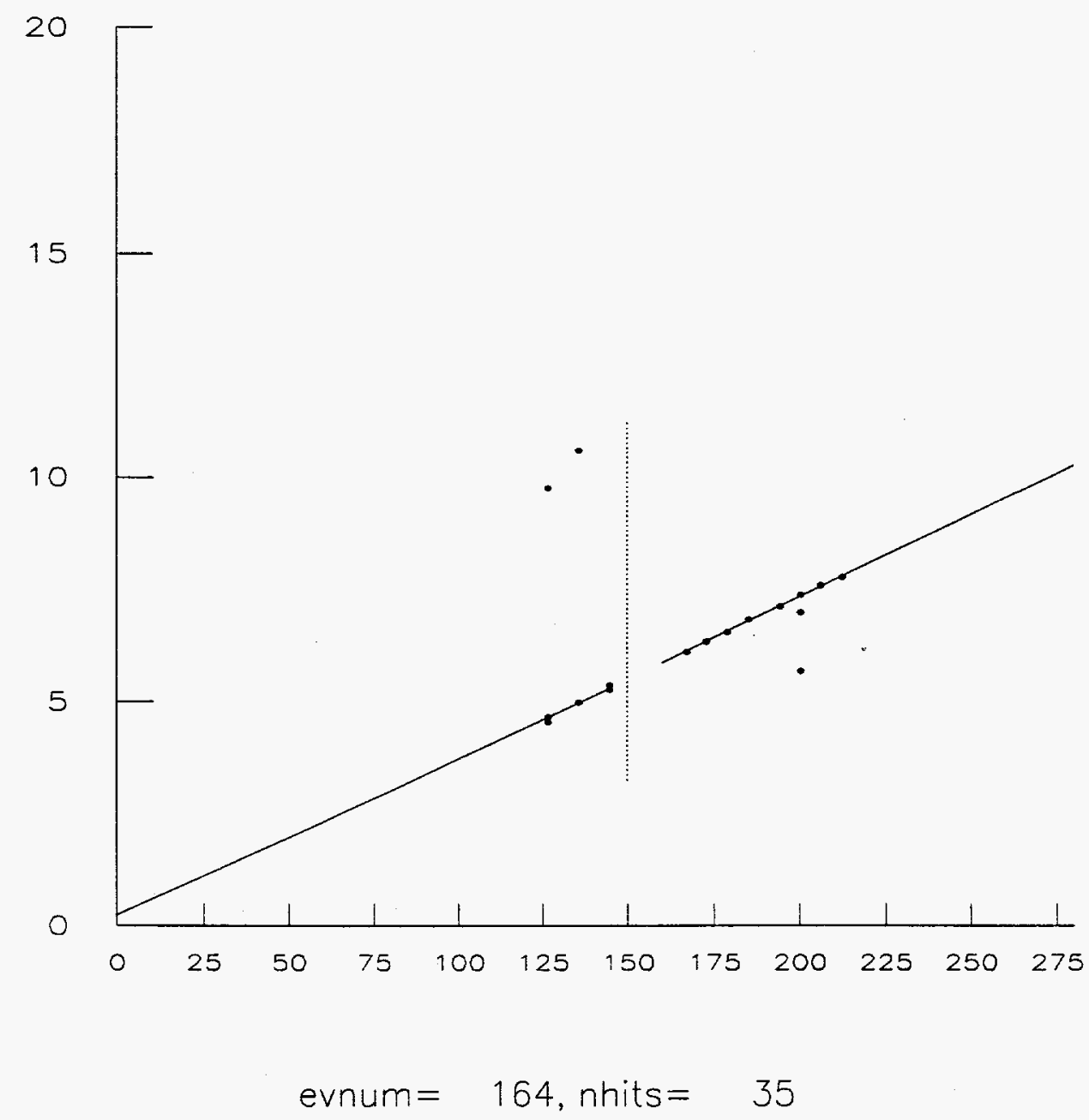

Figure 4.1: Event display of a clean charged track. 


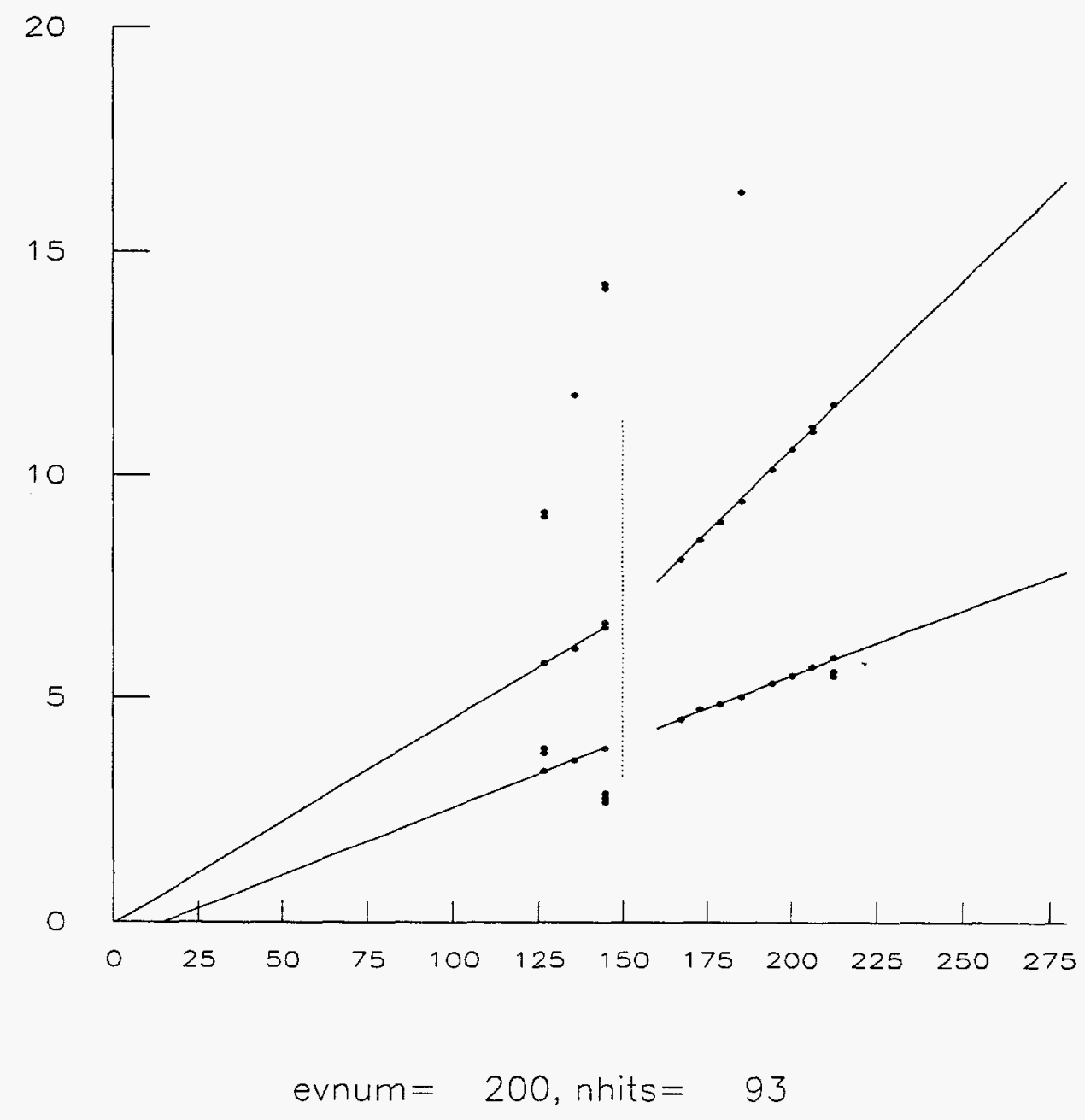

Figure 4.2: Event display of a straight-through charged track and a charged track which bends in the lead. 


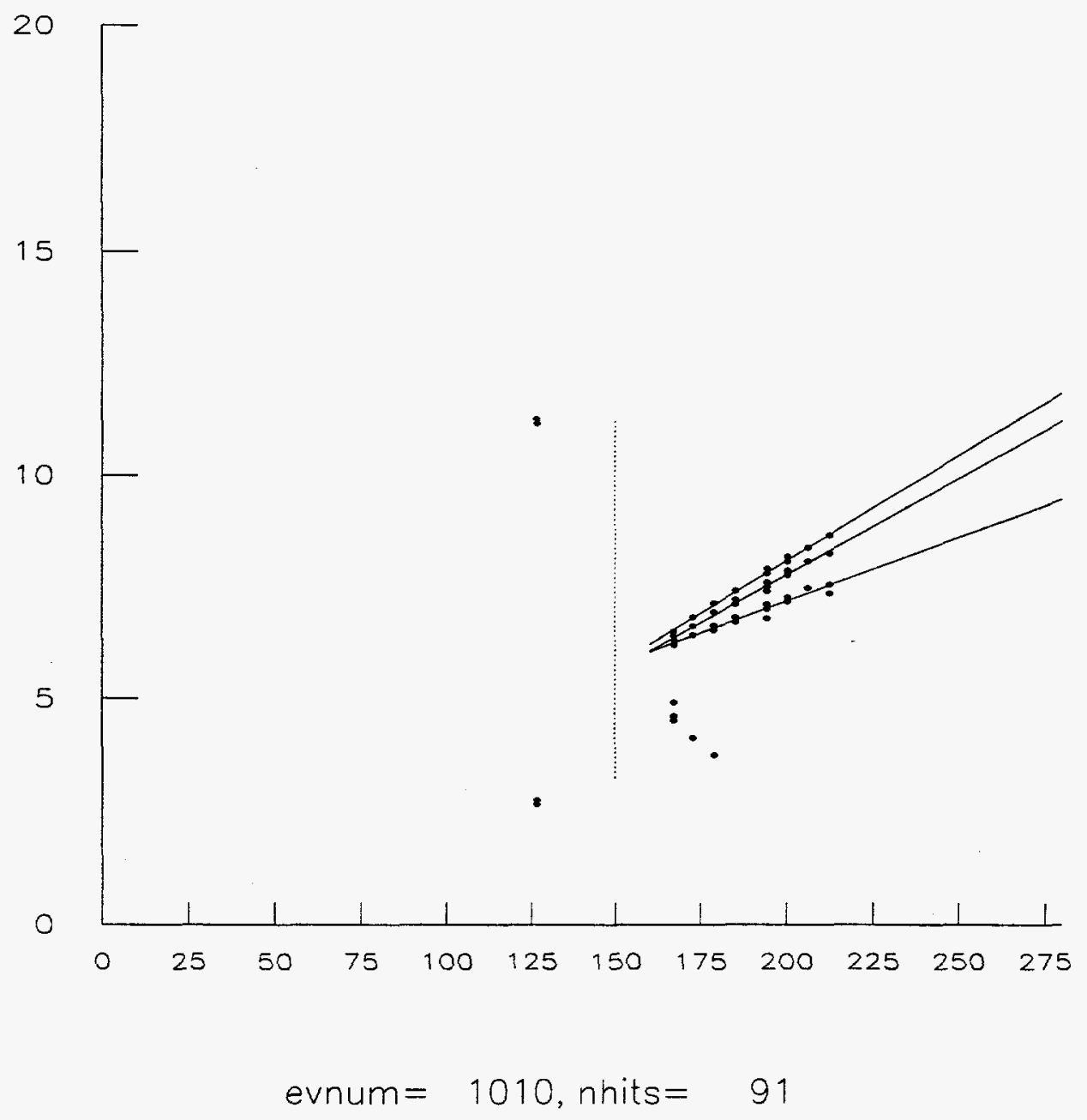

Figure 4.3: Event display of a photon with three conversion tracks. 


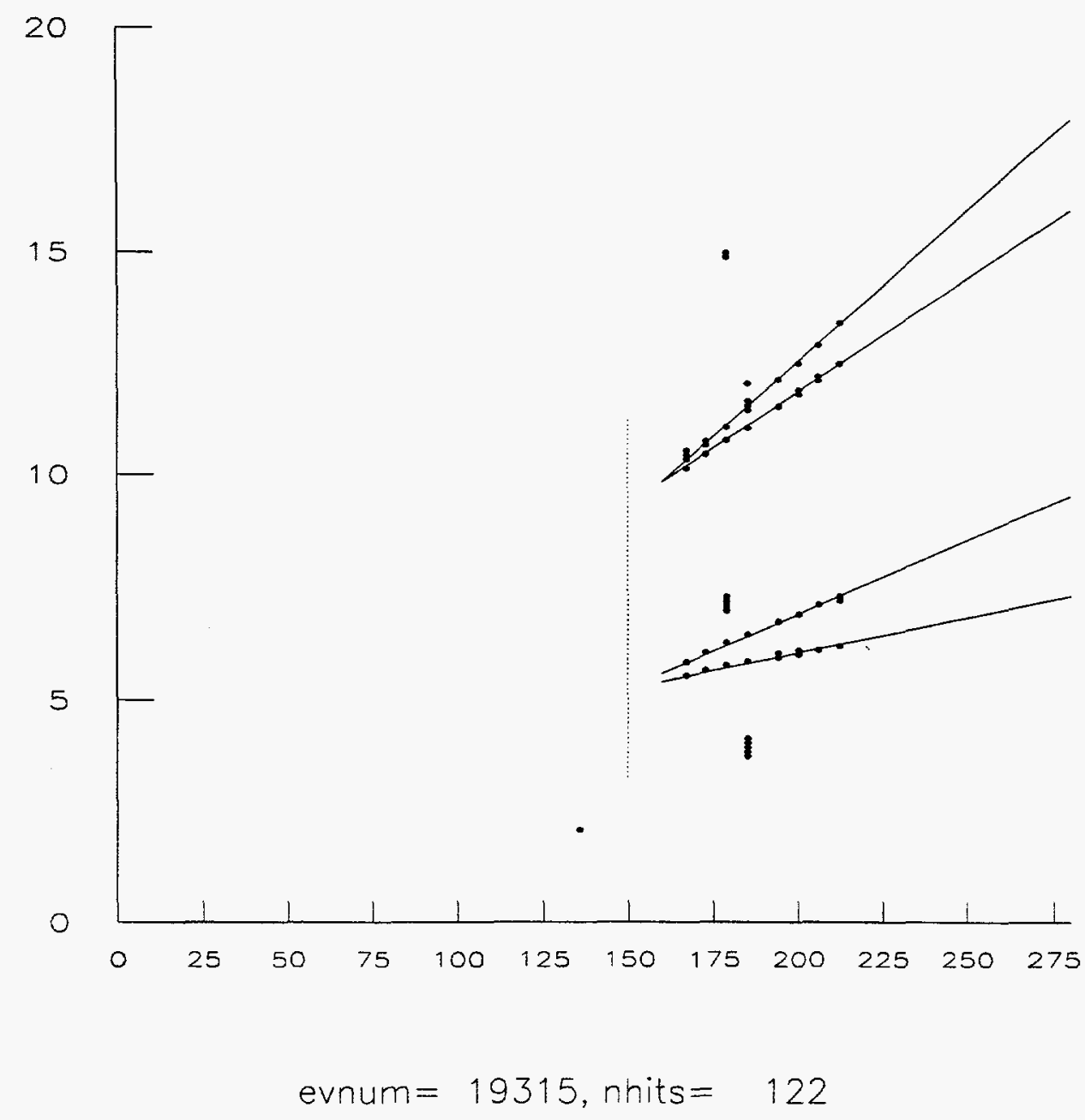

Figure 4.4: Event display of two clean photon conversions. 


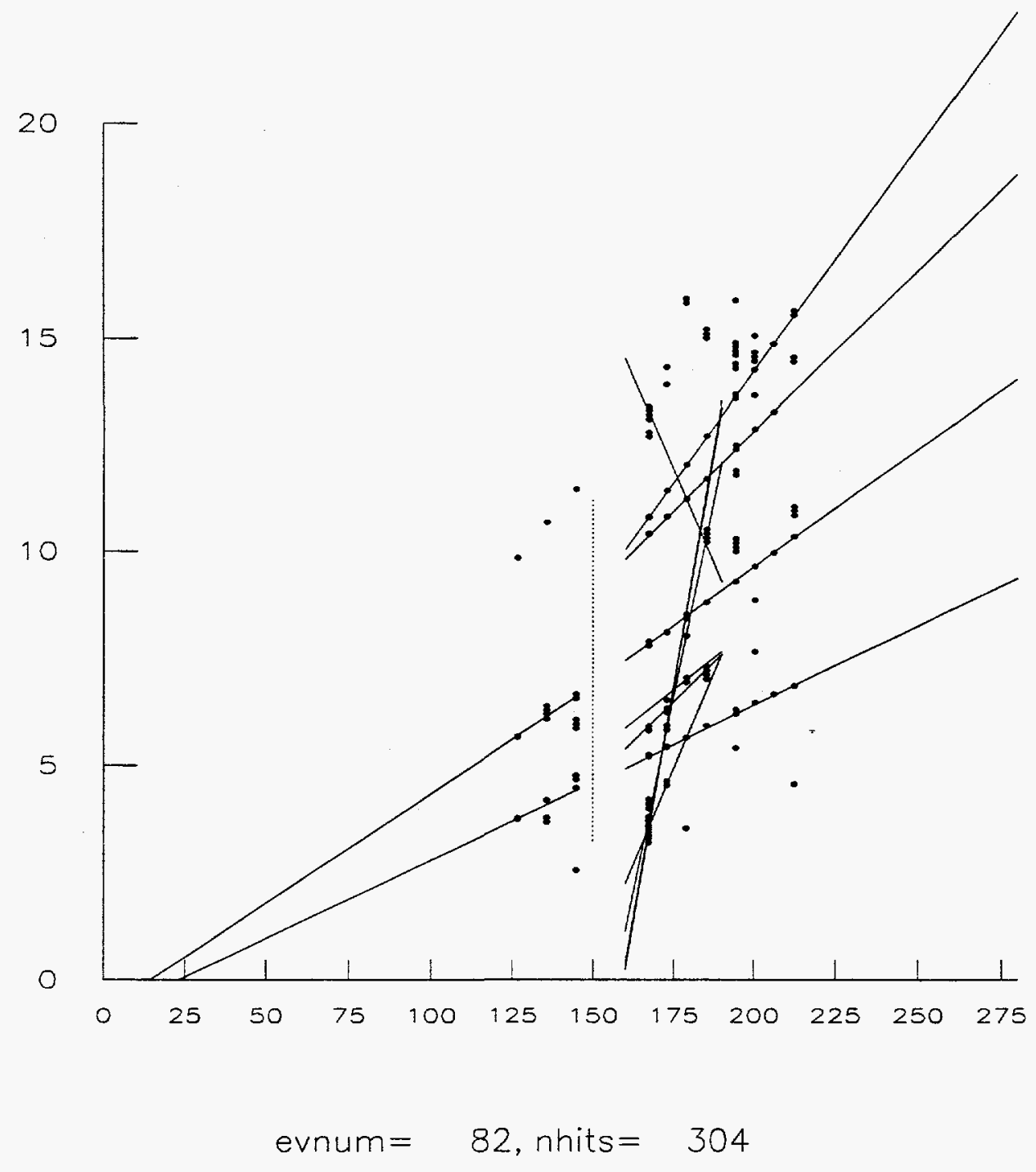

Figure 4.5: Display of an event with two charged tracks and a photon, and typical NHITS. 


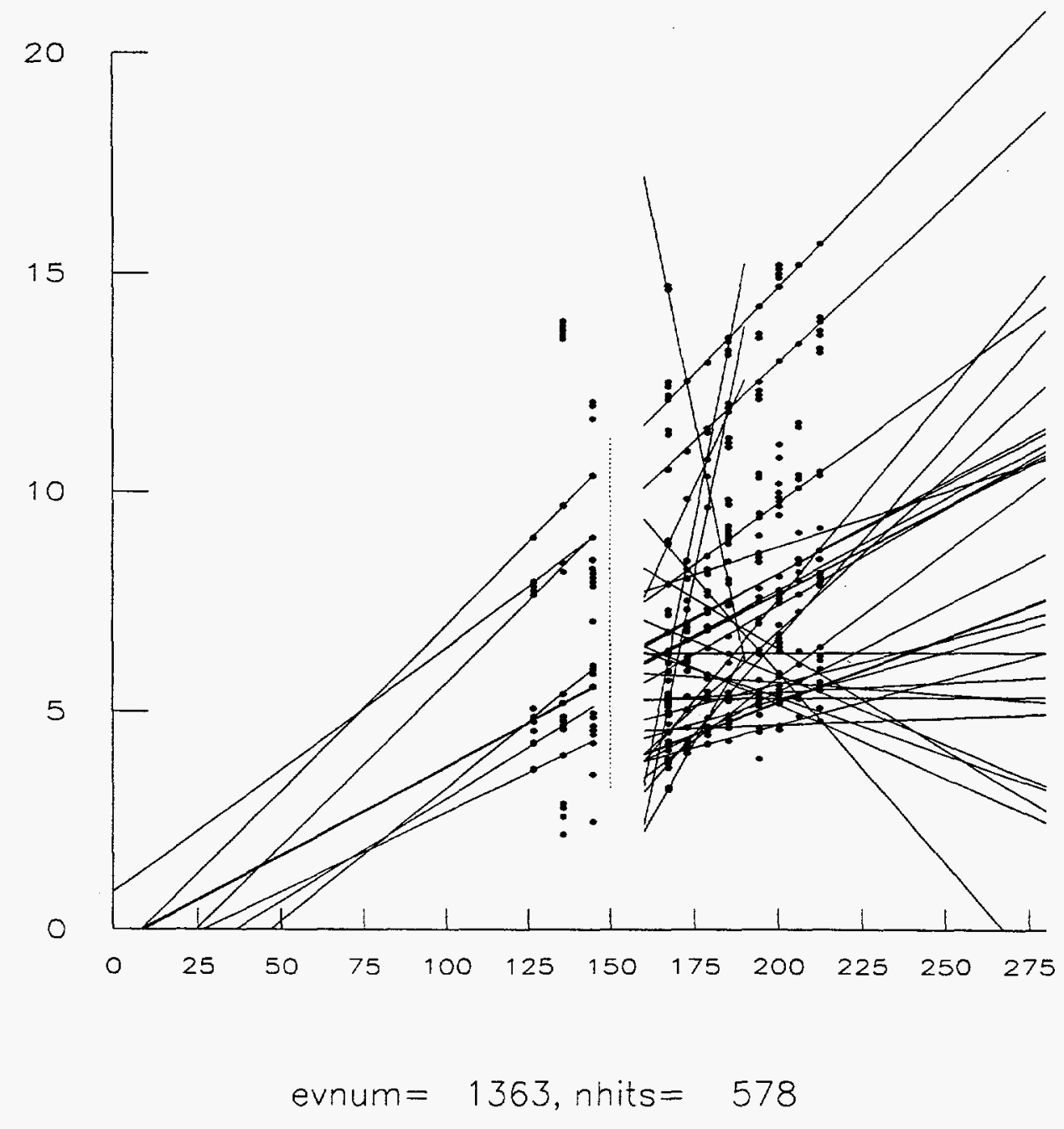

Figure 4.6: Display of an event with a large NHITS. 


\begin{tabular}{rcccccccccccc}
164 & \multicolumn{1}{c}{1} & 1 & & & & & & & & \\
77 & 328 & 485 & 610 & 694 & 870 & 927 & & & & & & \\
& 15 & & & & & & & & & & \\
1055 & 1249 & 1377 & 1440 & 1633 & 1761 & 1888 & 1955 & 2144 & 2273 & 2337 & 2655 \\
2730 & 2850 & 2979 & & & & & & & & &
\end{tabular}

\begin{tabular}{ccccccccccccc}
1010 & \multicolumn{3}{c}{0} & 3 & & & & & & & & \\
\multicolumn{1}{c}{15} & & & & & & & & & & \\
1058 & 1241 & 1374 & 1436 & 1629 & 1763 & 1884 & 1965 & 2140 & 2264 & 2341 & 2650 \\
2745 & 2855 & 2977 & & & & & & & & & \\
19 & & & & & & & & & & \\
1057 & 1056 & 1241 & 1242 & 1376 & 1433 & 1632 & 1766 & 1888 & 1960 & 2144 & 2269 \\
2335 & 2533 & 2656 & 2737 & 2846 & 2848 & 2969 & & & & & \\
1059 & 1239 & 1372 & 1439 & 1627 & 1758 & 1882 & 1965 & 2136 & 2264 & 2344 & 2647 \\
2741 & 2859 & 2986 & & & & & & & & &
\end{tabular}

Figure 4.7: Entries in the dst for the events shown in Figs. 4.1 and 4.3. 


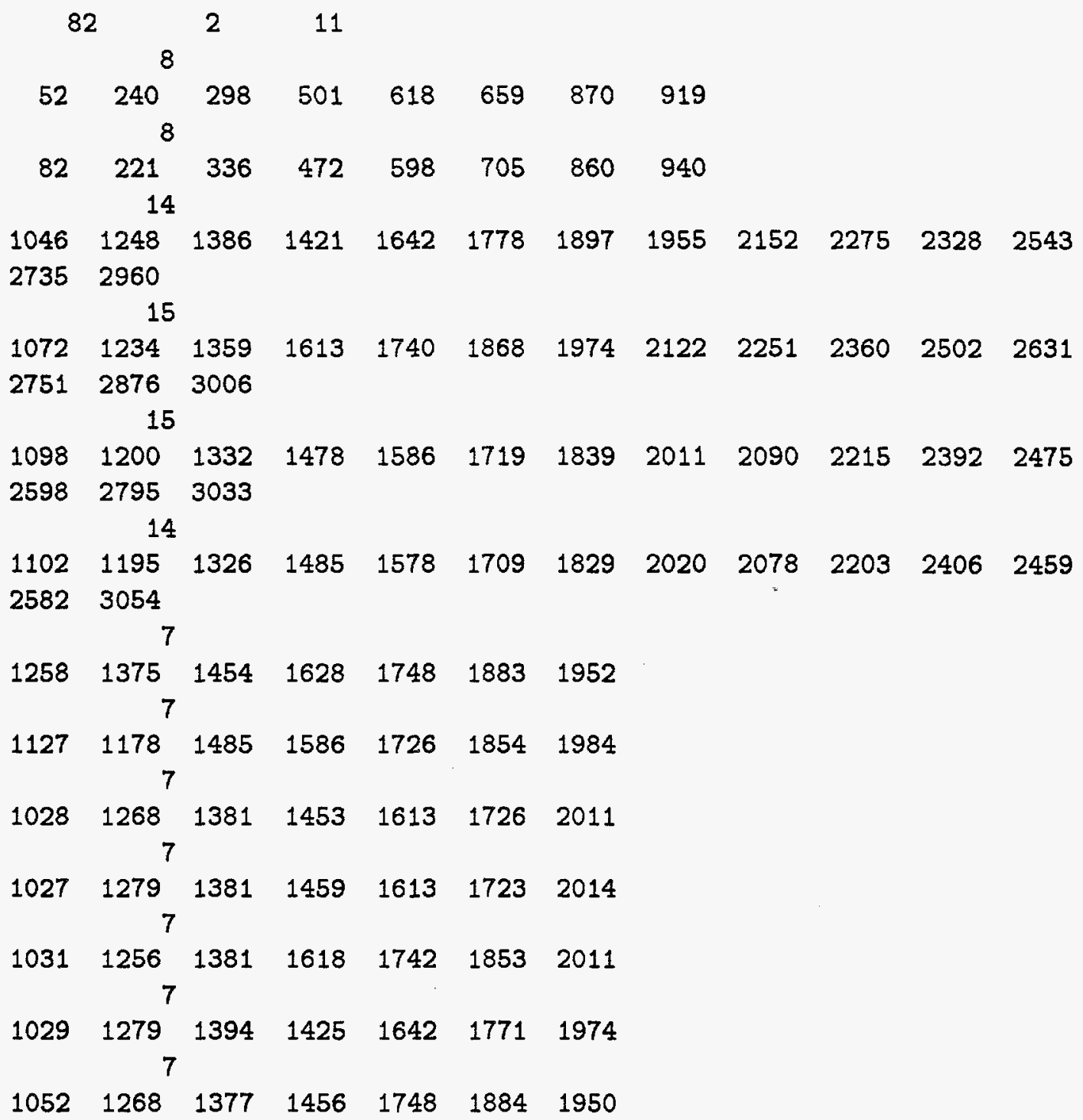

Figure 4.8: Entry in the dst for the event shown in Fig. 4.5. 


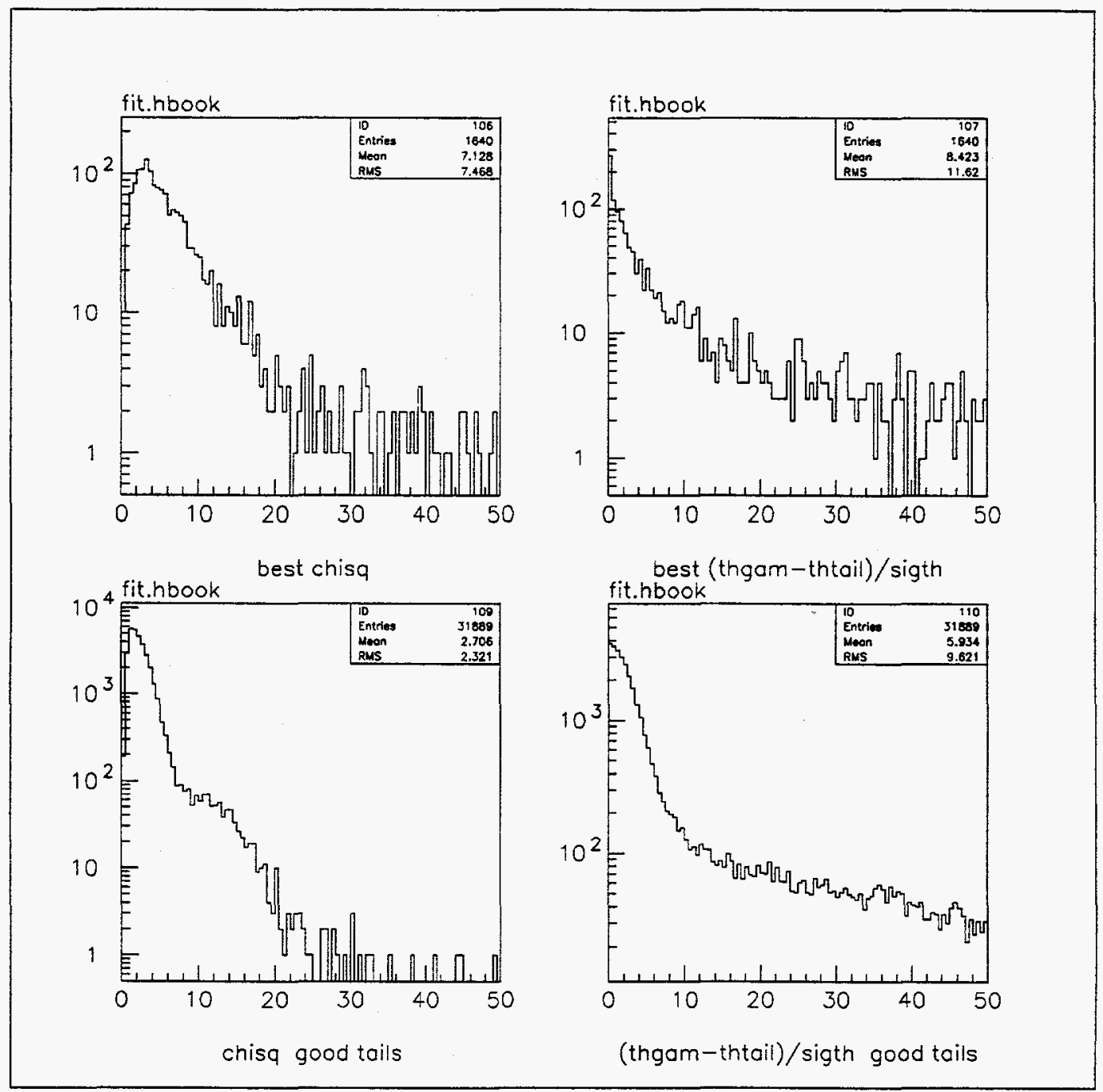

Figure 4.9: Histograms of $\chi^{2}$ for the fit in $v$ and of $\left|\theta_{0}-\theta\right| / \sigma_{\theta}$ for re-fitted tails and good tails. Cuts were made of $\chi^{2}<7$ and $\left|\theta_{0}-\theta\right| / \sigma_{\theta}<8$. 


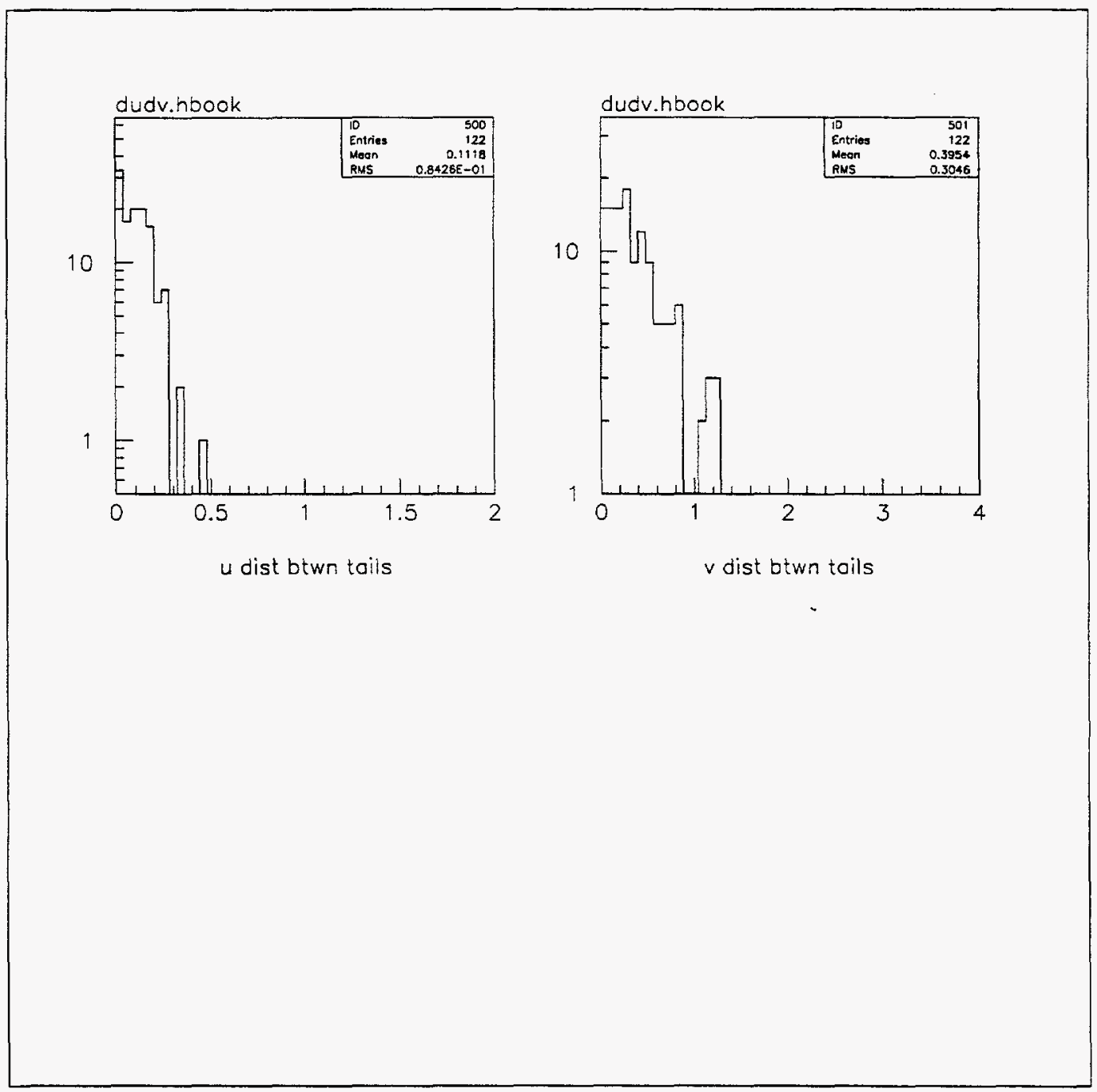

Figure 4.10: Distance in $u$ and $v$ between photon conversion tracks at the lead when there are two conversion tracks in GEANT. 

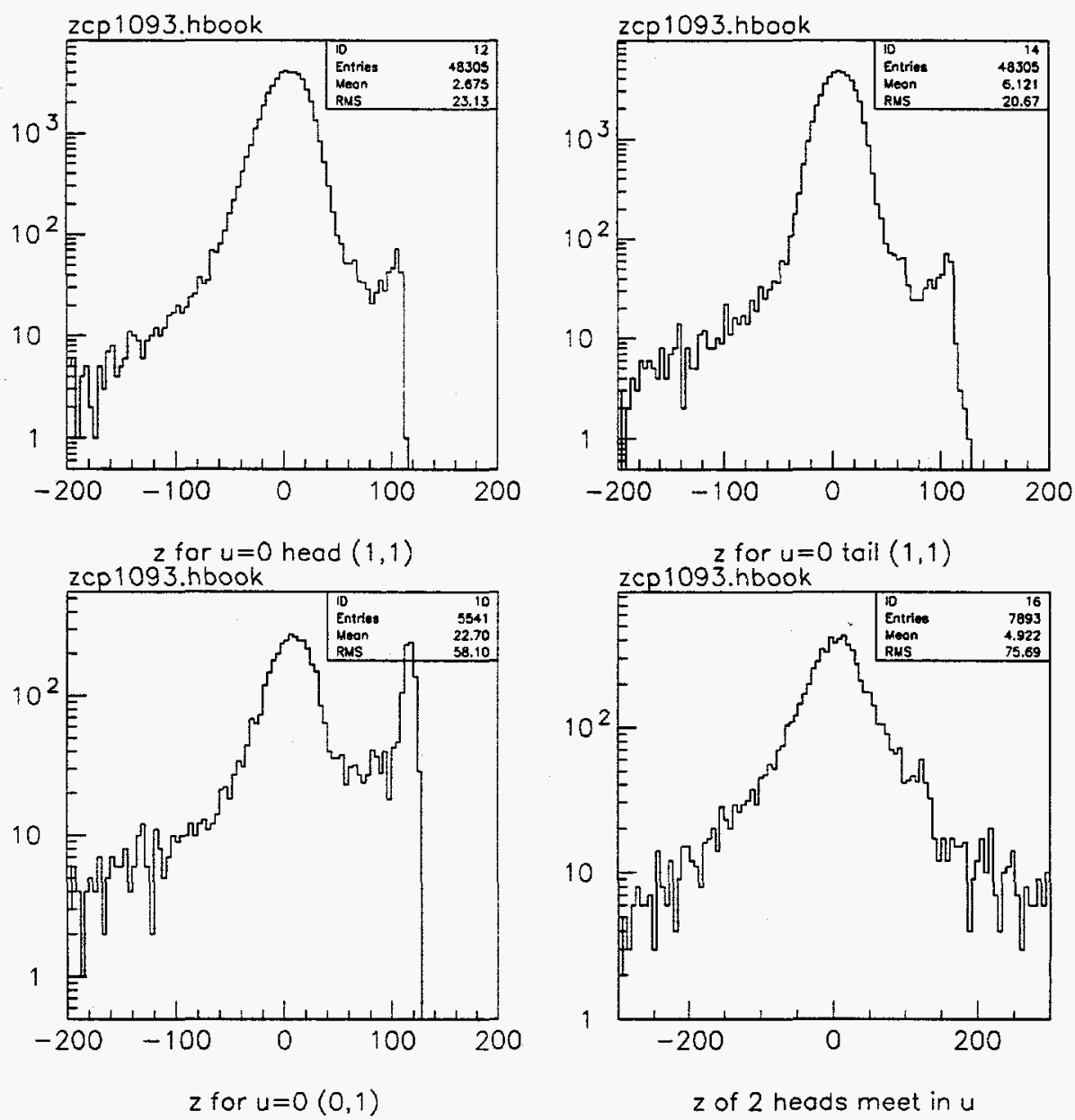

Figure 4.11: Pointing, defined as the $z$ where the track goes through $u=0$, for heads and tails of charged tracks, single photon conversion tracks, and the $z$ where any two heads have the same $u$ position, for lead-out run 1093. 


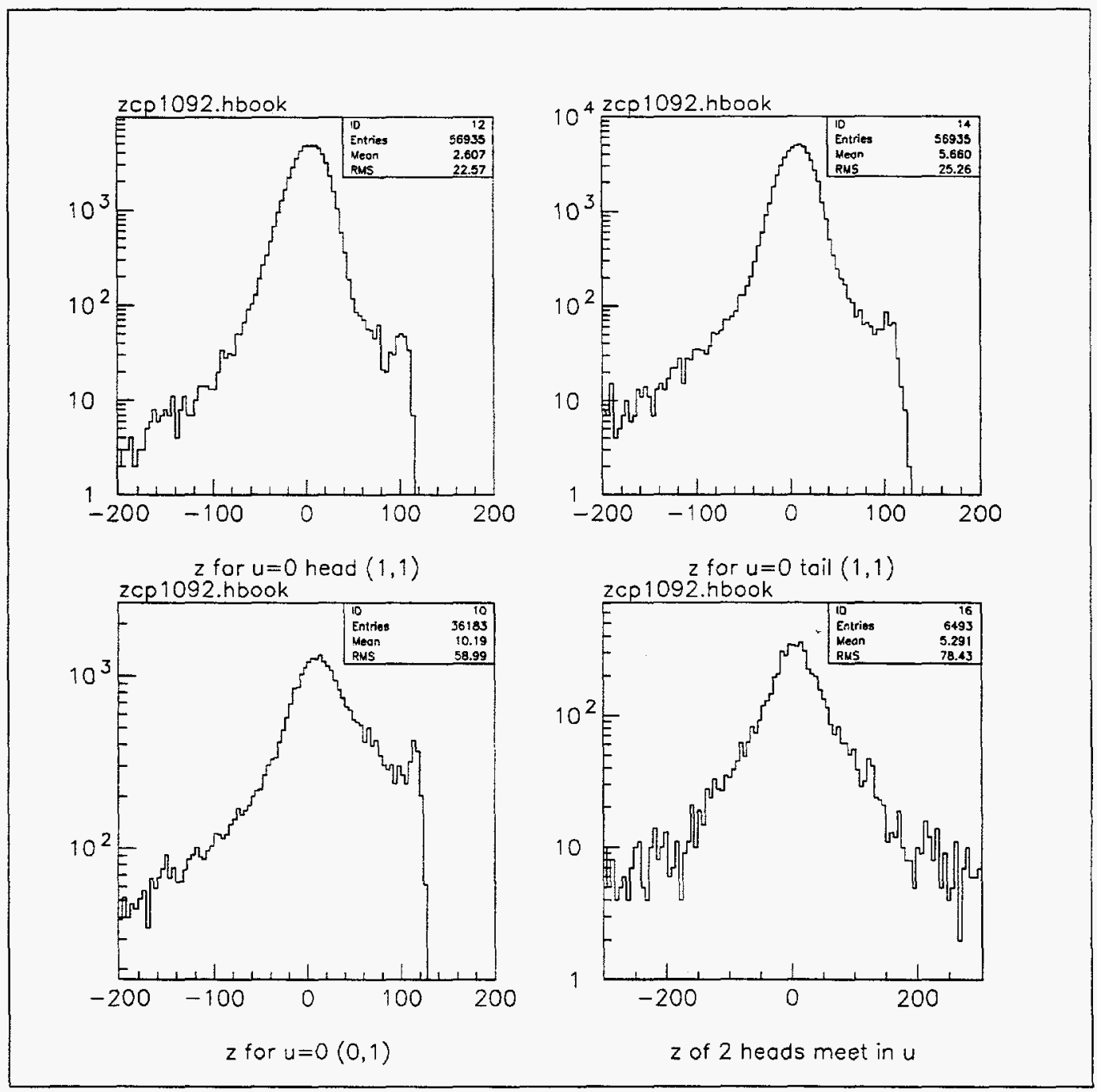

Figure 4.12: Pointing, defined as the $z$ where the track goes through $u=0$, for heads and tails of charged tracks, single photon conversion tracks, and the $z$ where any two heads have the same $u$ position, for lead-in run 1092. 


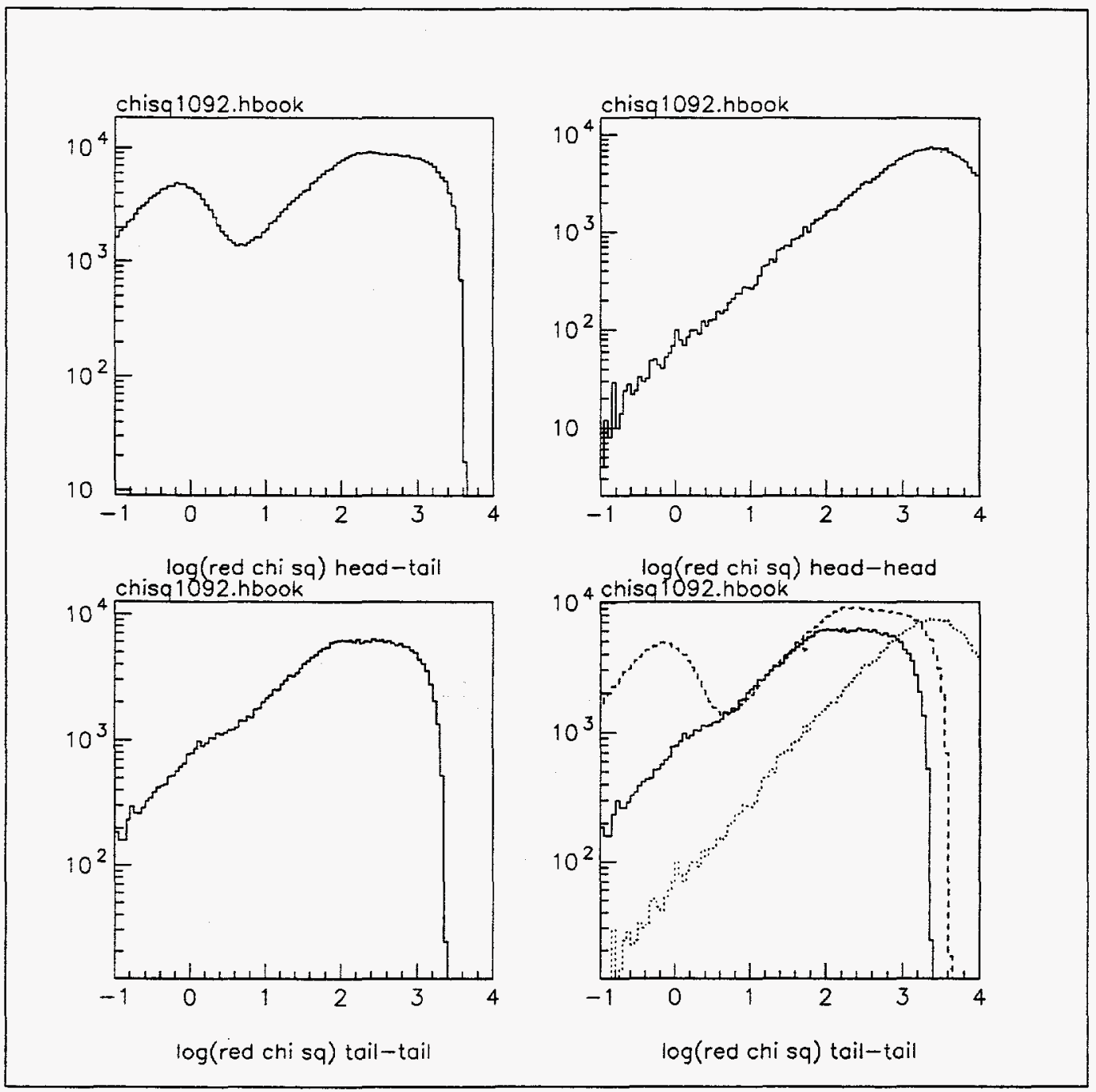

Figure 4.13: Values of $\log \frac{\chi^{2}}{2}$ for head-tail, head-head, and tail-tail matches at the lead. A superposition of these plots shows that $\log \frac{x^{2}}{2}=0.7$, or $\frac{x^{2}}{2}=5$ is a reasonable cutoff between matching real tracks together and track segments which are coincidentally close at the lead. 


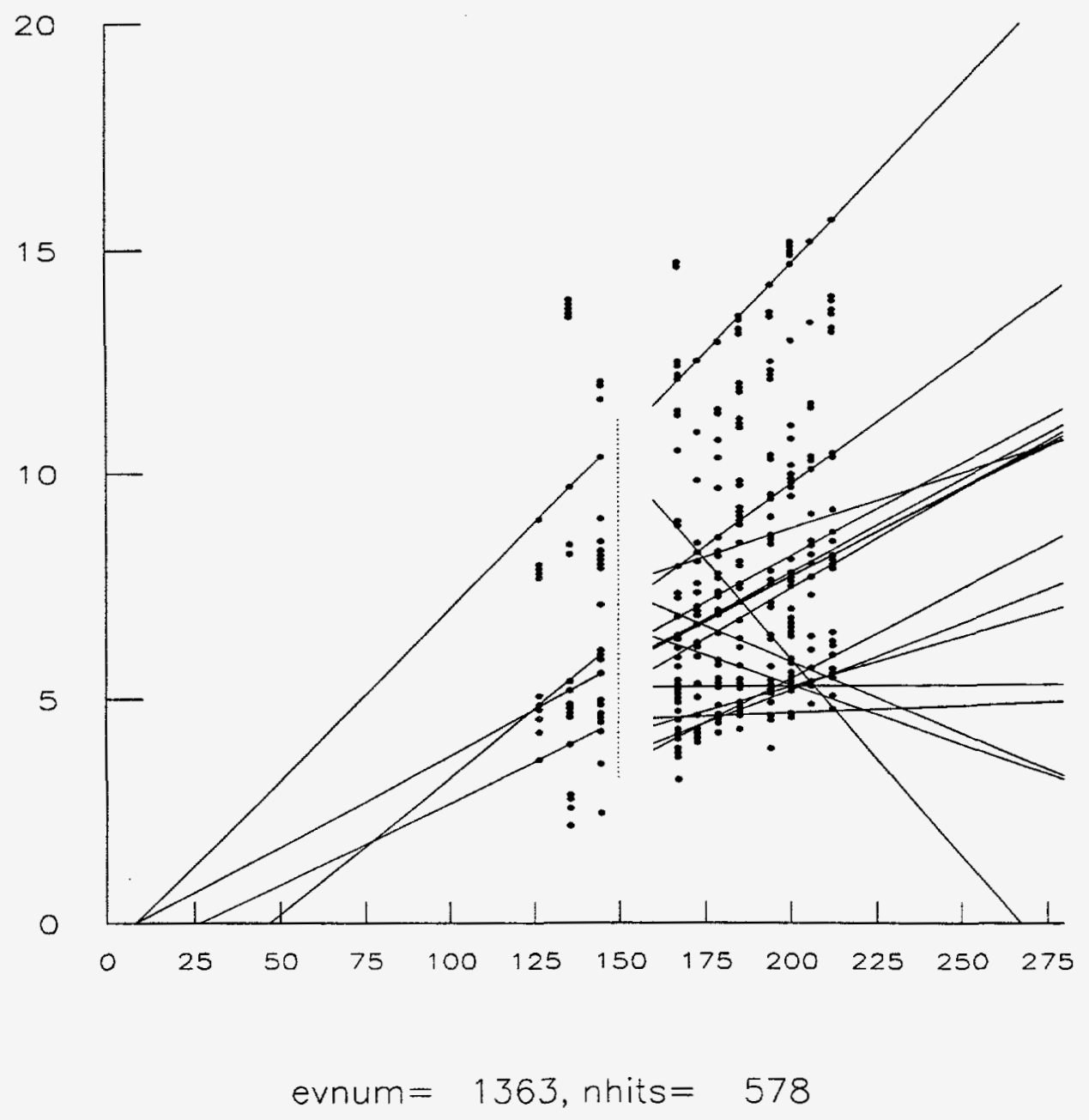

Figure 4.14: Display of the event shown in Fig. 4.6 for tracks kept by the vertexer. 


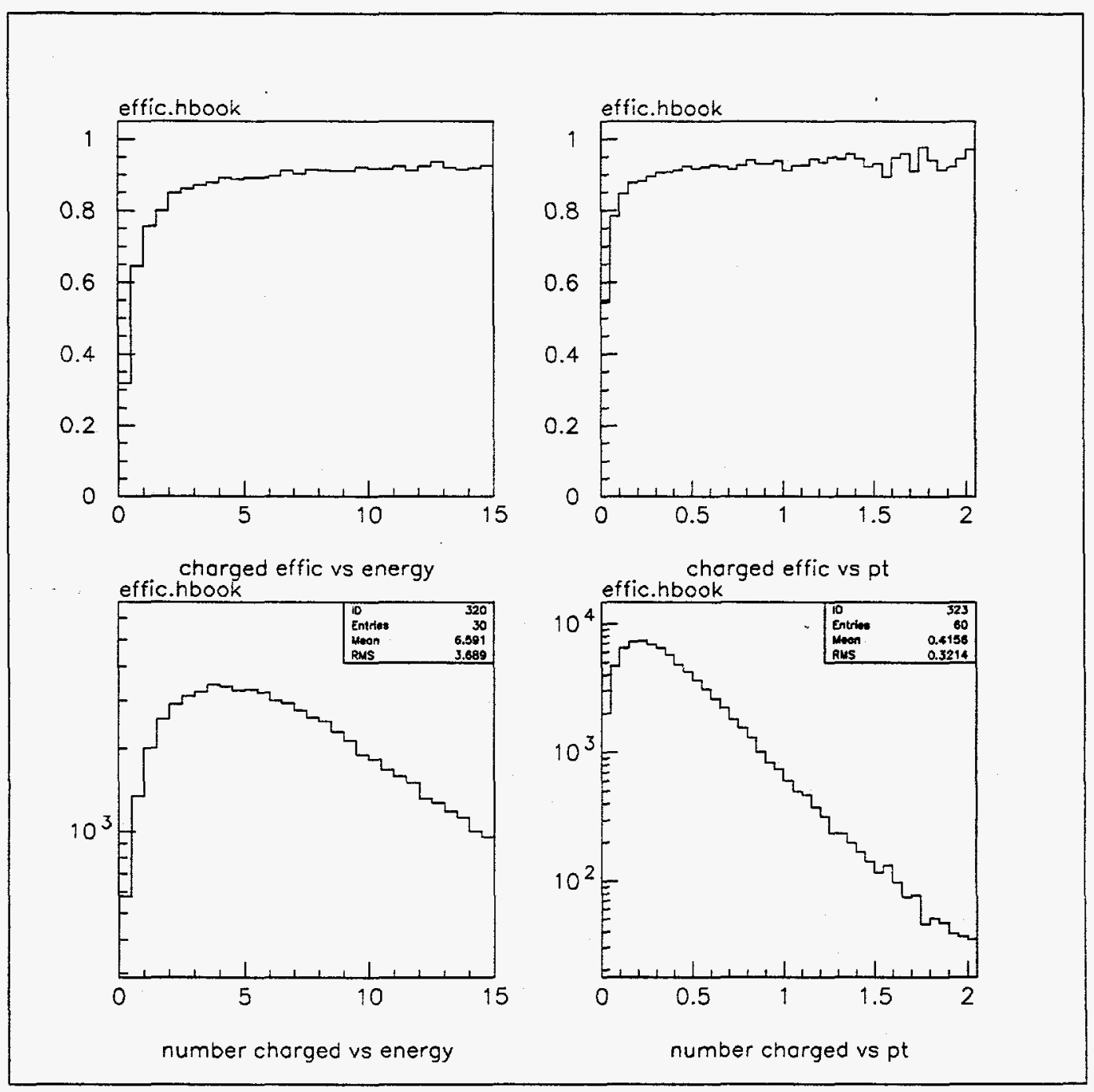

Figure 4.15: Efficiencies for finding charged tracks as a function of energy and transverse momentum of the track, number of charged tracks vs energy, transverse momentum. 


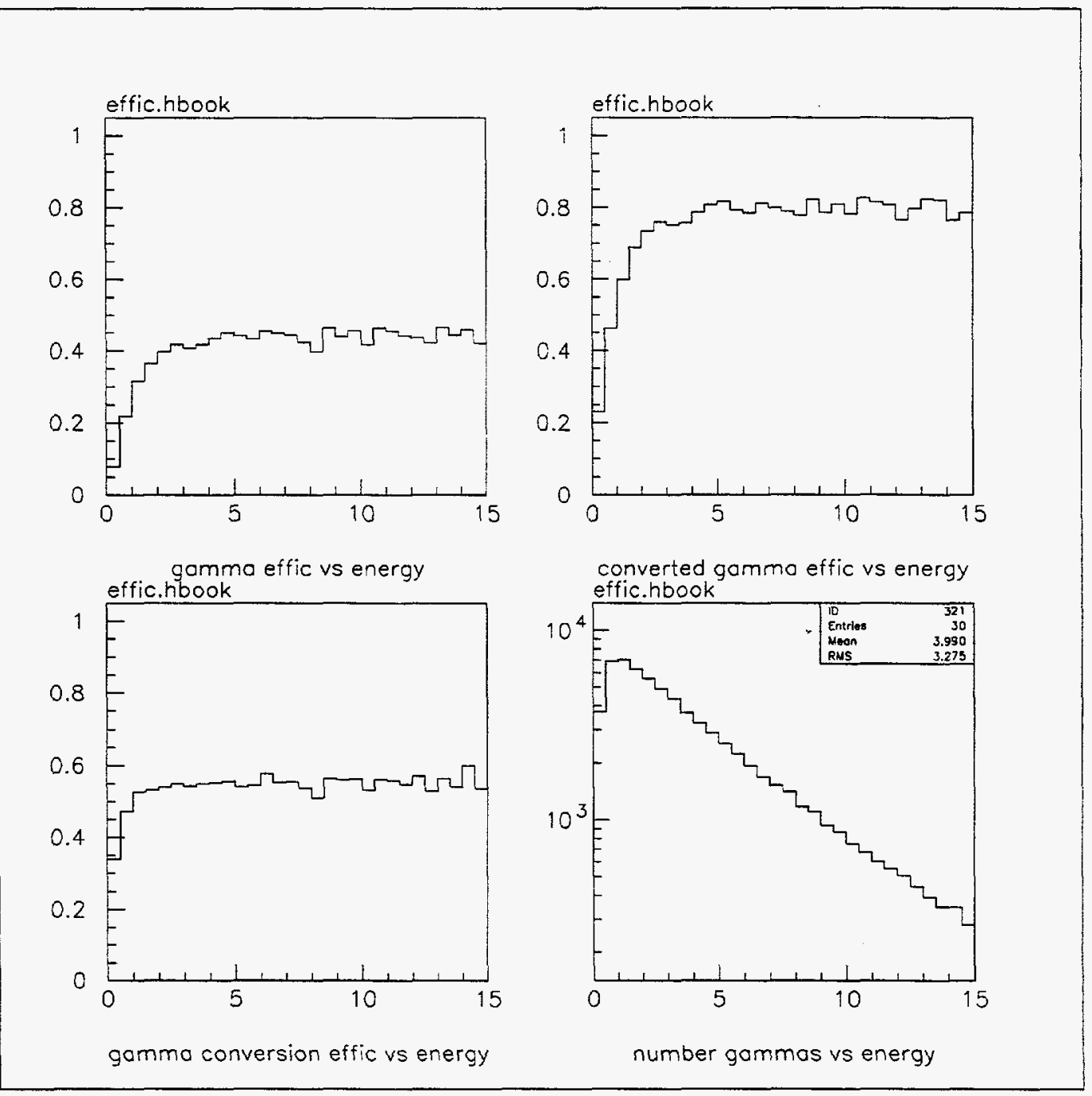

Figure 4.16: Efficiency for finding photons as a function of photon energy, efficiency for finding photons know to convert vs energy, probability of conversion of a photon vs energy, number of photons vs energy. 


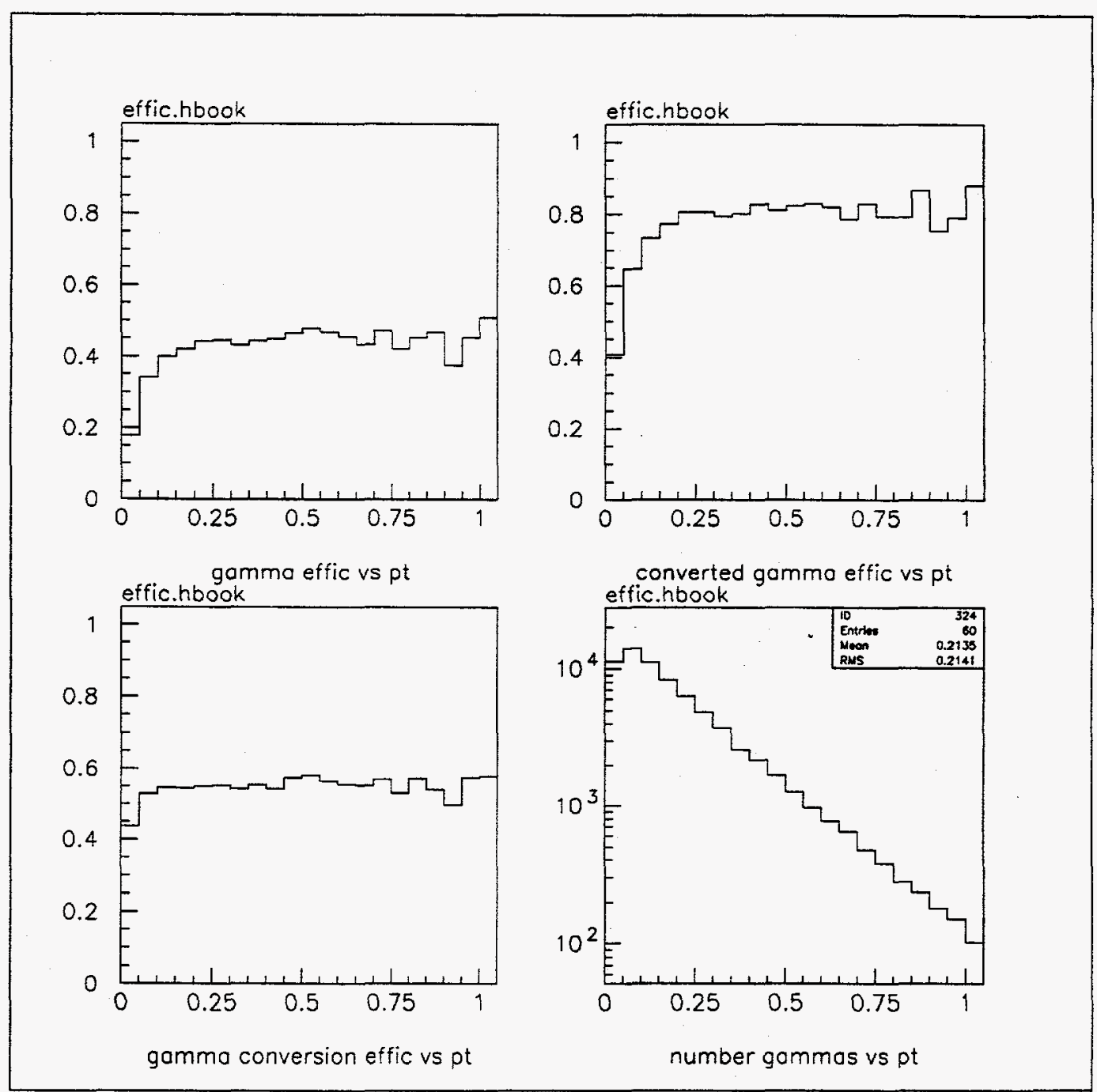

Figure 4.17: Efficiency for finding photons as a function of photon transverse momentum, efficiency for finding photons know to convert vs $p_{T}$, probability of conversion of a photon vs $p_{T}$, number of photons vs $p_{T}$. 


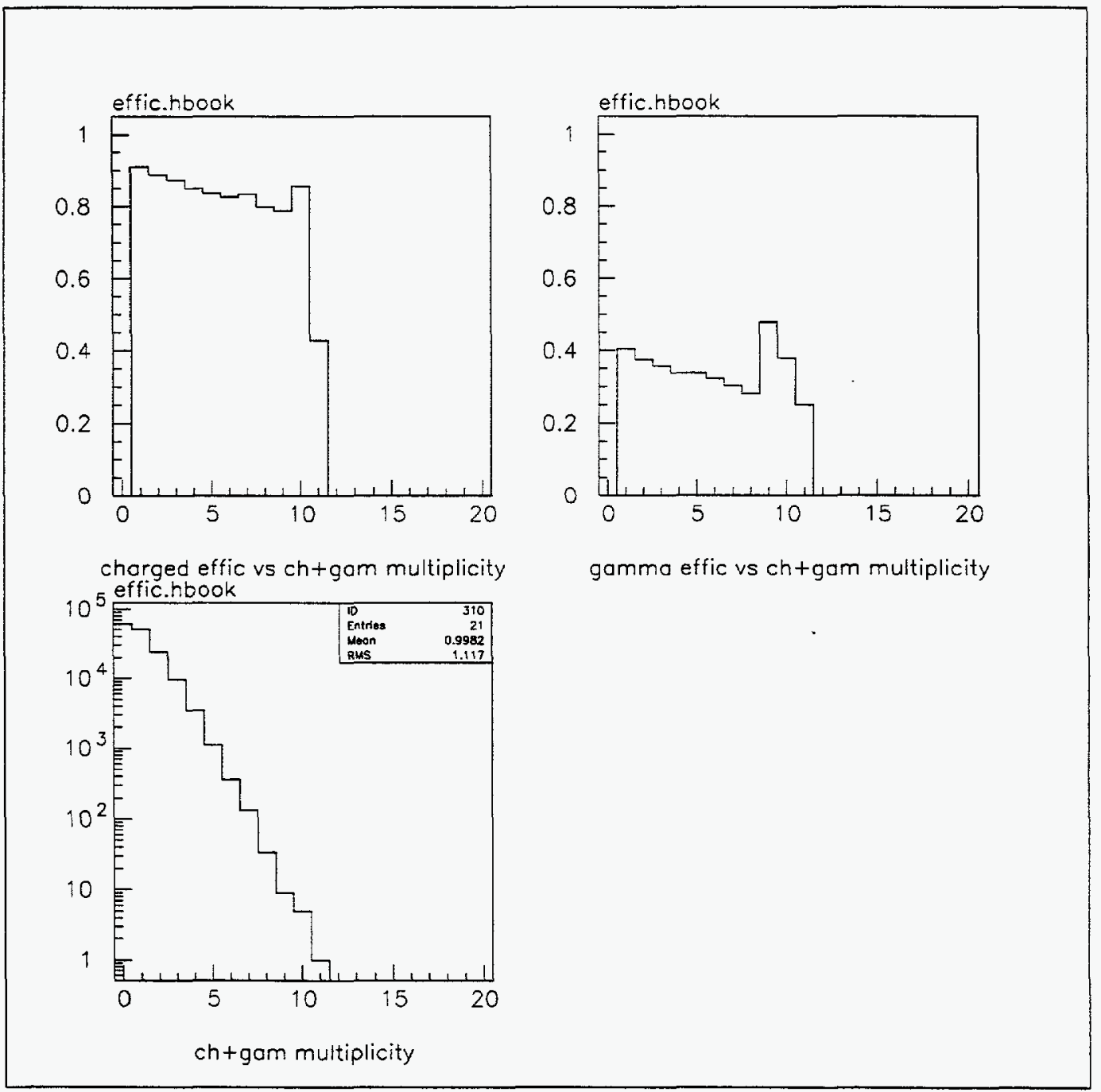

Figure 4.18: Efficiency for finding charged tracks as a function of total multiplicity into the acceptance, efficiency for finding photons vs total multiplicity, frequency of observing an event with a given total multiplicity. 


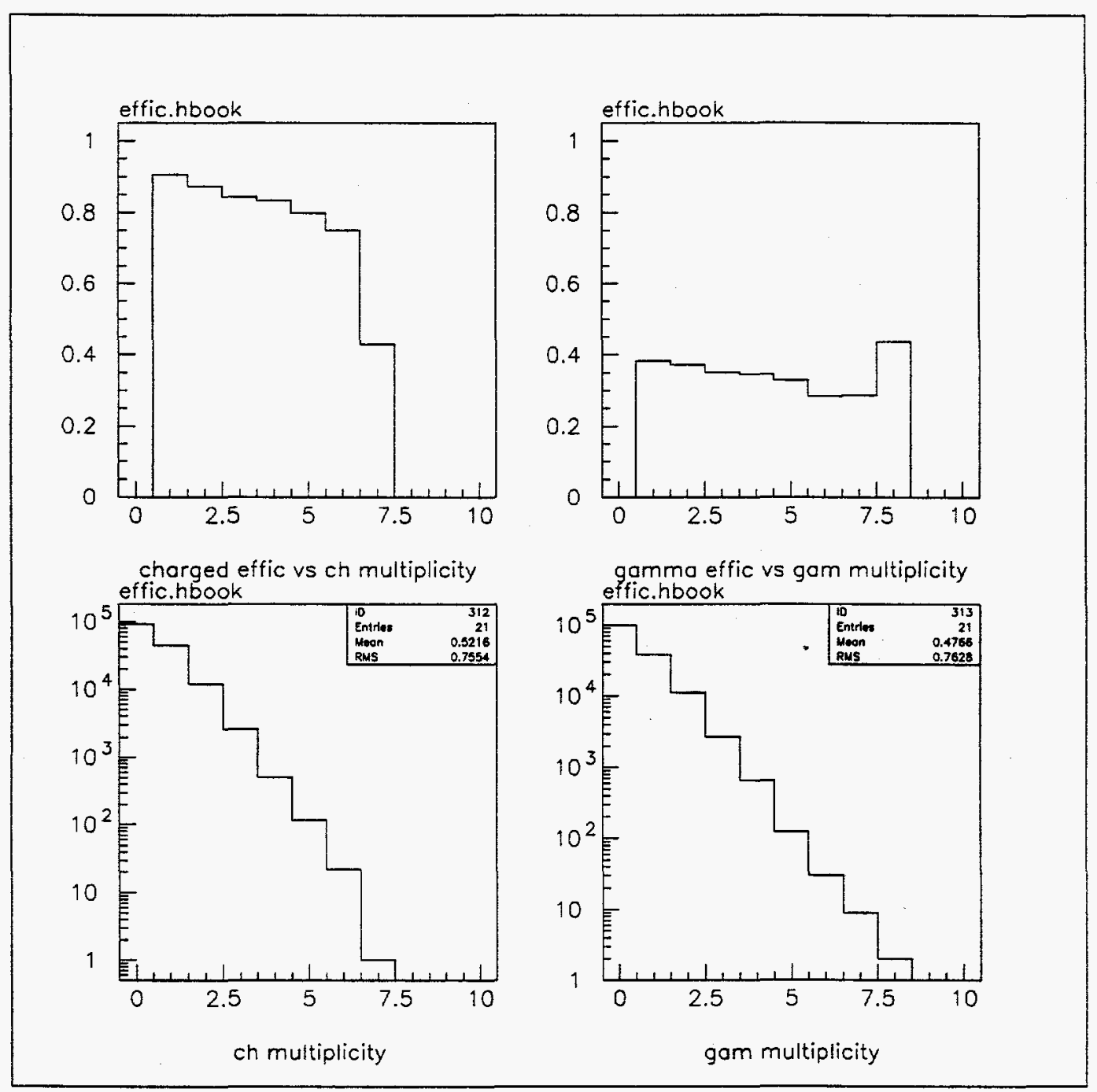

Figure 4.19: Efficiency for finding charged tracks as a function of charged multiplicity into the acceptance, efficiency for finding photons vs photon multiplicity, frequency of observing an event with a given charged multiplicity, frequency vs photon multiplicity. 


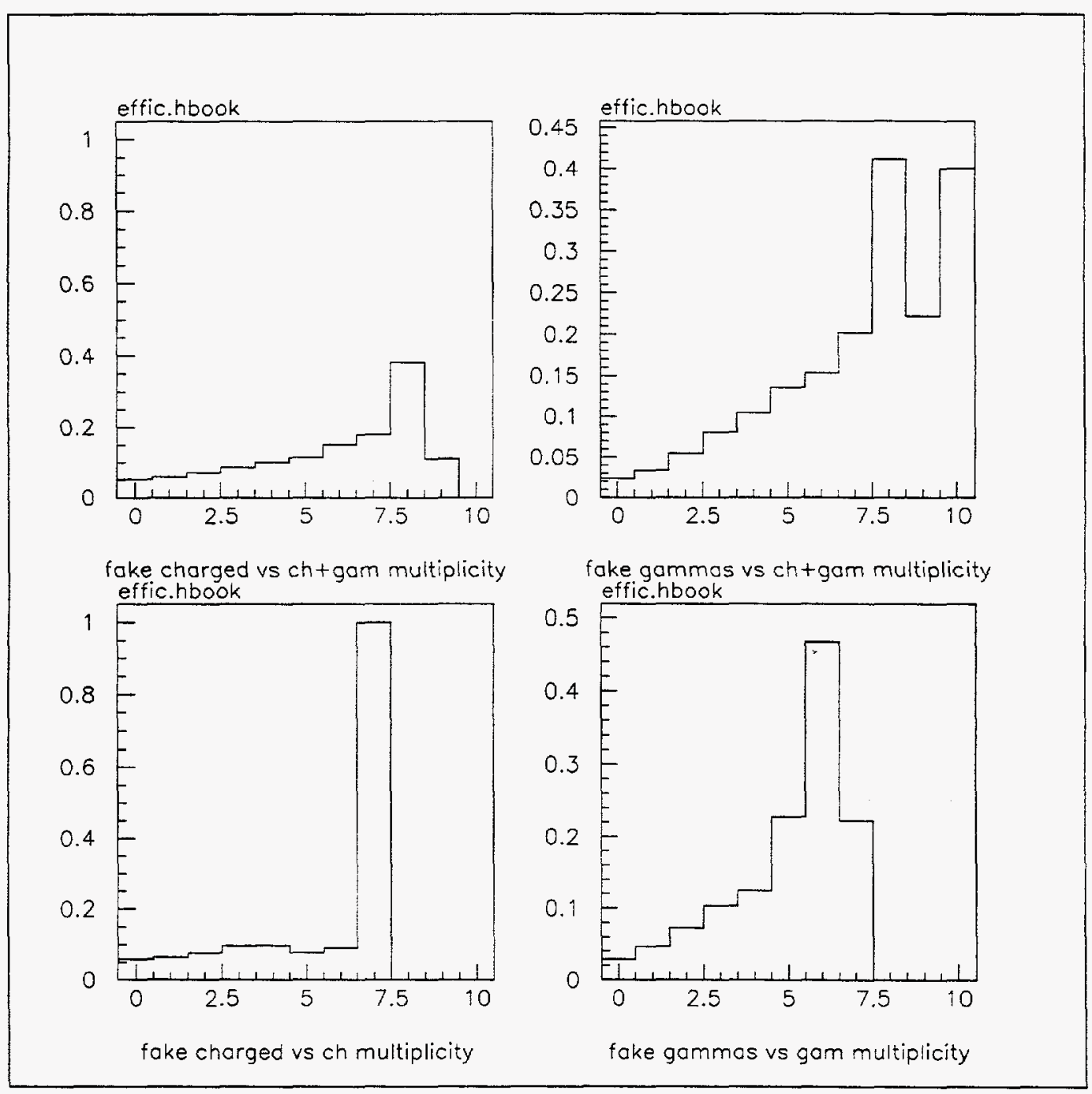

Figure 4.20: Mean number of fake charged tracks found per event with a given total multiplicity, mean number of fake photons found per event with a given photon multiplicity, mean number of fake charged tracks vs charged multiplicity, mean number of fake photons vs photon multiplicity. 


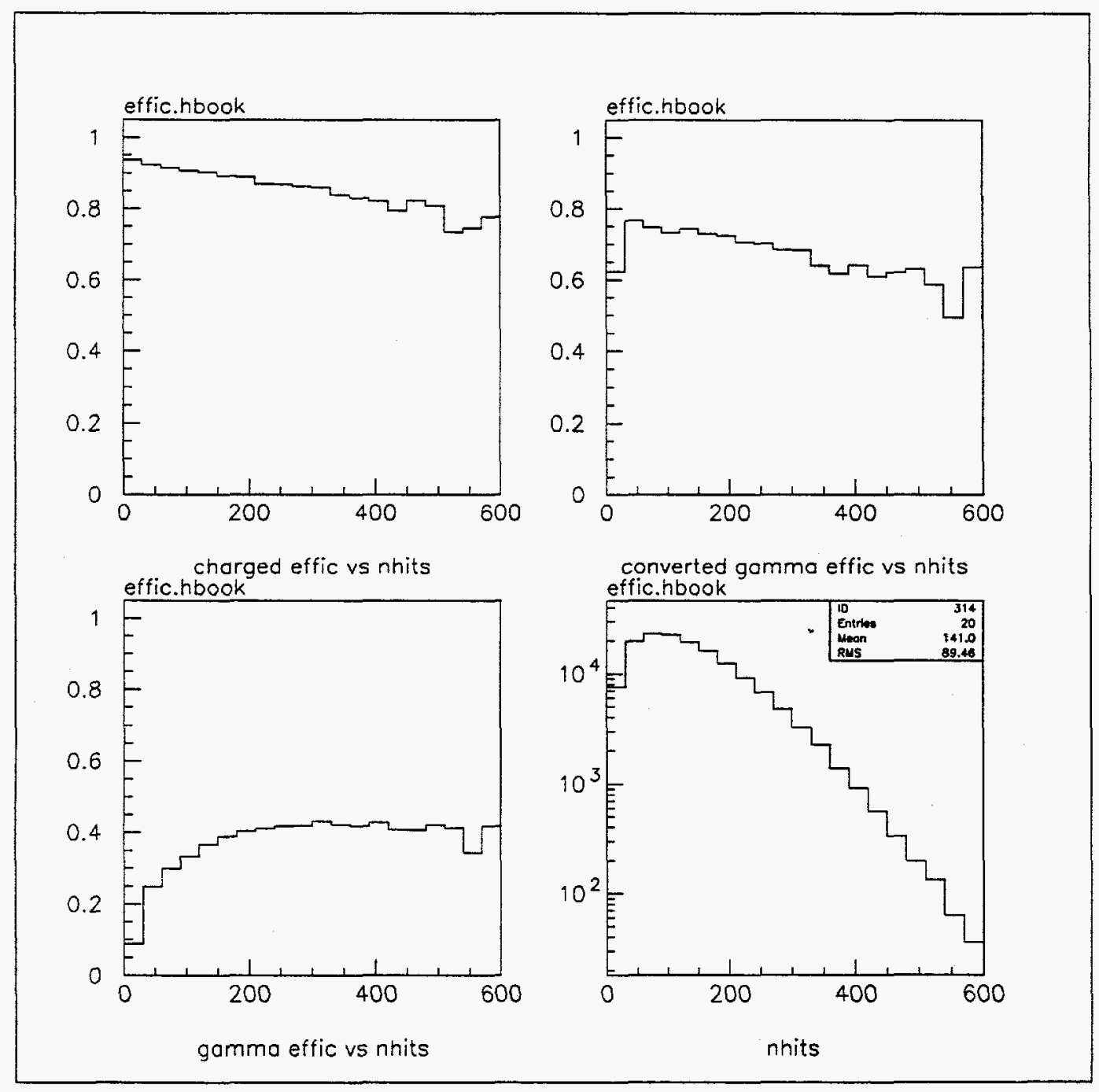

Figure 4.21: Efficiency for finding charged tracks as a function of NHITS of the event, efficiencies for finding converted photons and all photons vs NHITS, frequency of observing an event with a given NHITS. 


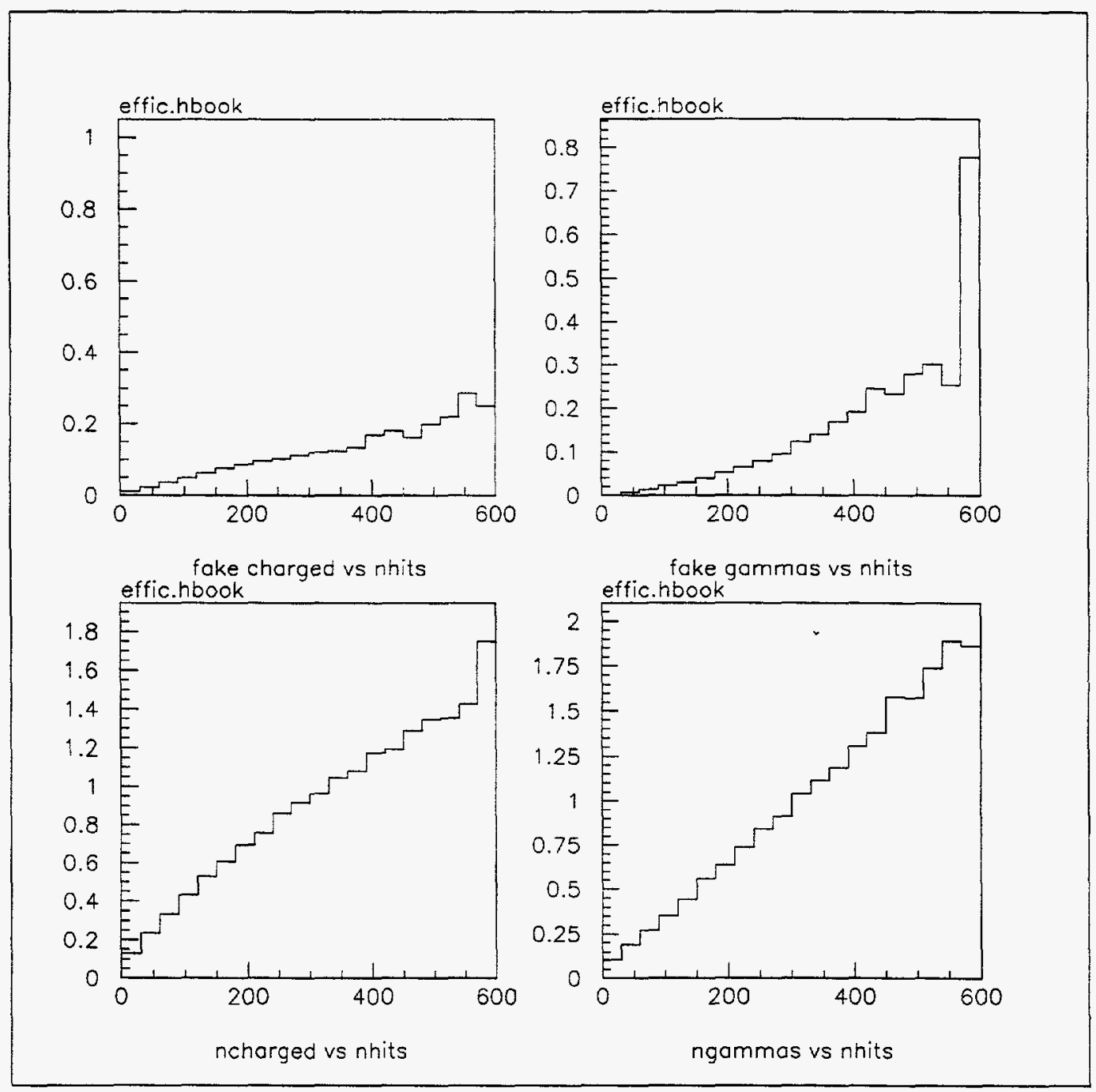

Figure 4.22: Mean number of fake charged tracks found per event with a given NHITS, mean number of fake photons found per event with a given NHITS, mean numbers of real charged tracks, real photons given NHITS. 


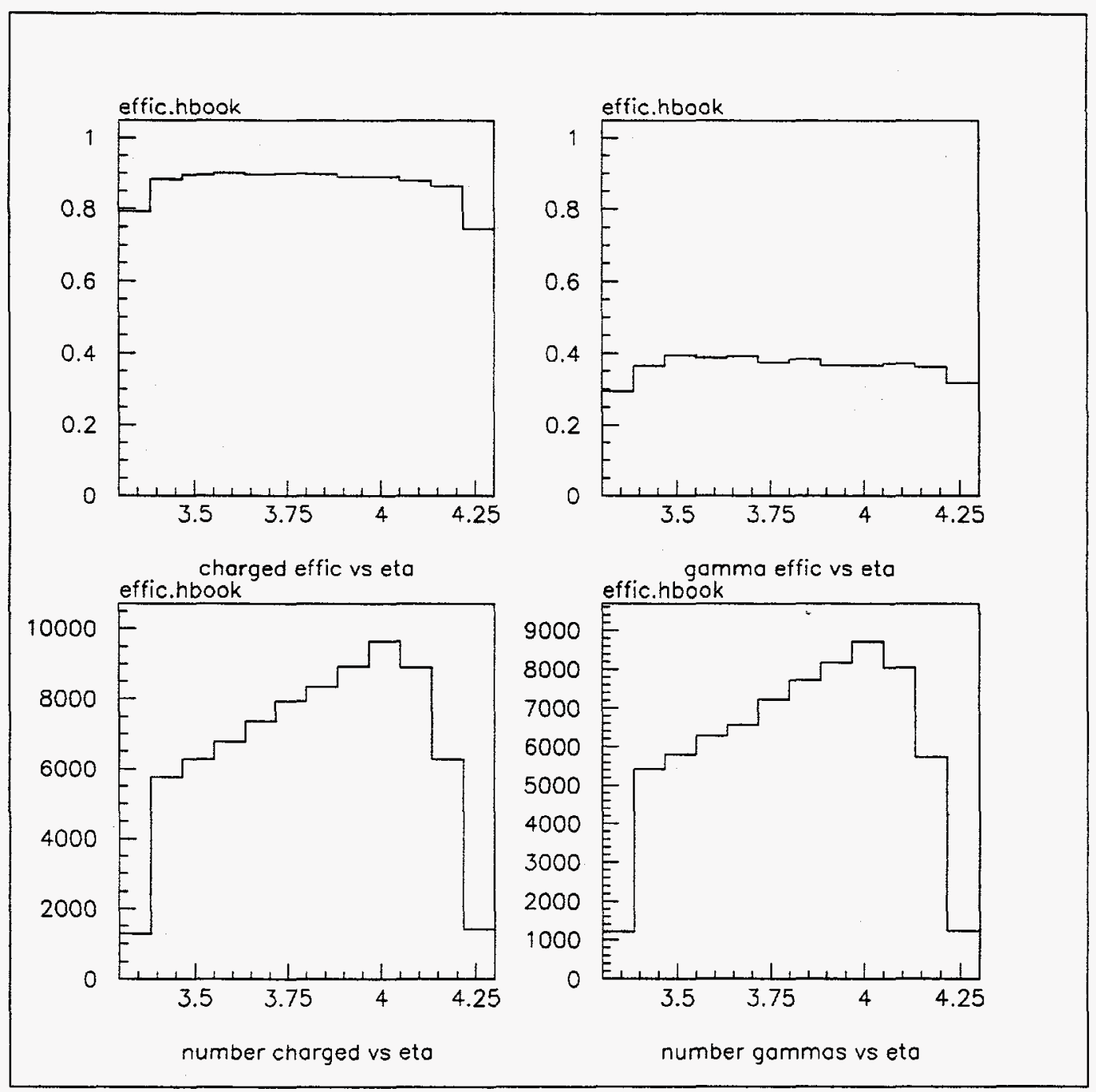

Figure 4.23: Efficiencies for finding charged tracks, photons at a given $\eta$, number of charged tracks, photons vs $\eta$. 


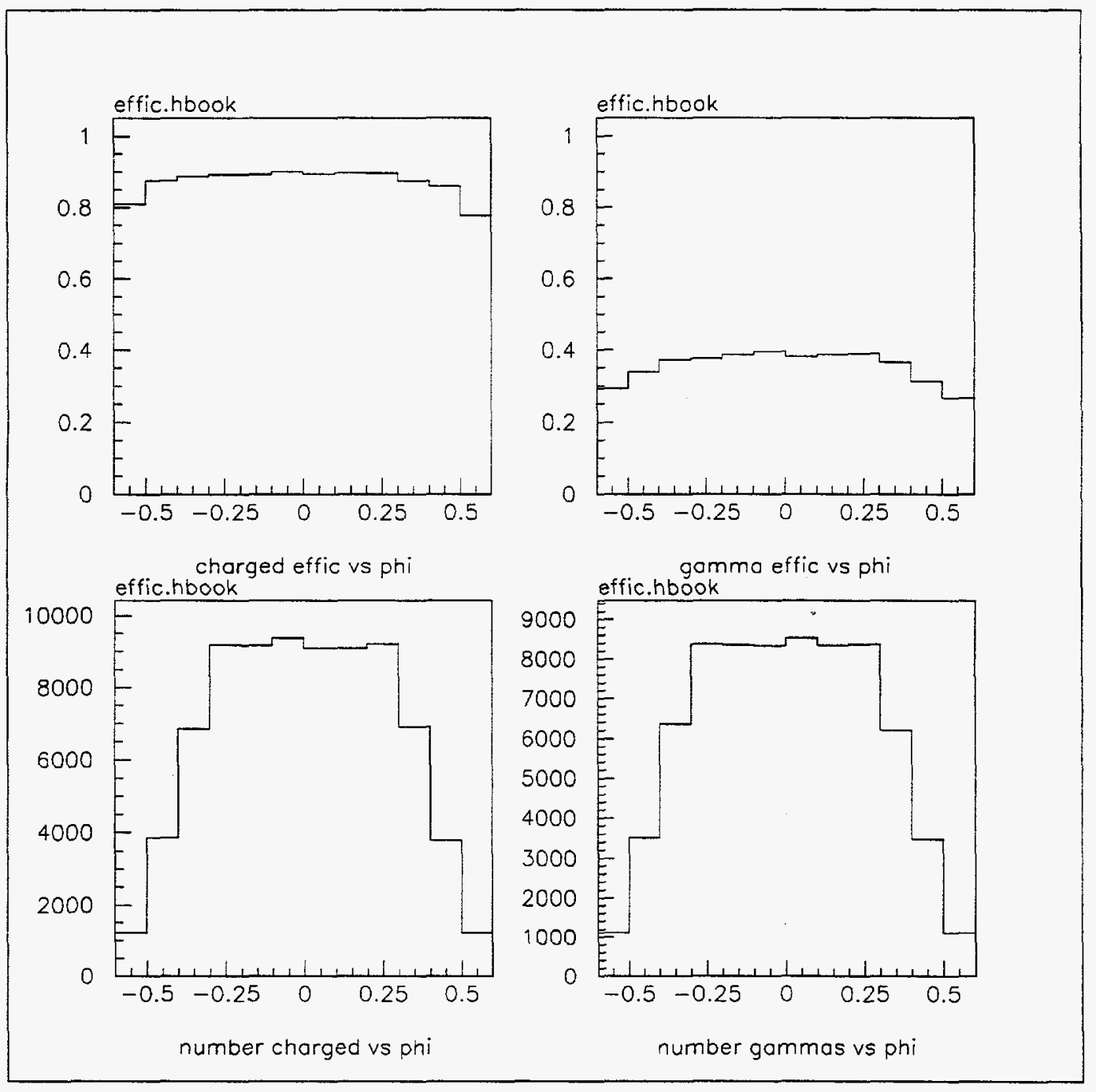

Figure 4.24: Efficiencies for finding charged tracks, photons at a given $\phi$, number of charged tracks, photons vs $\phi$. 


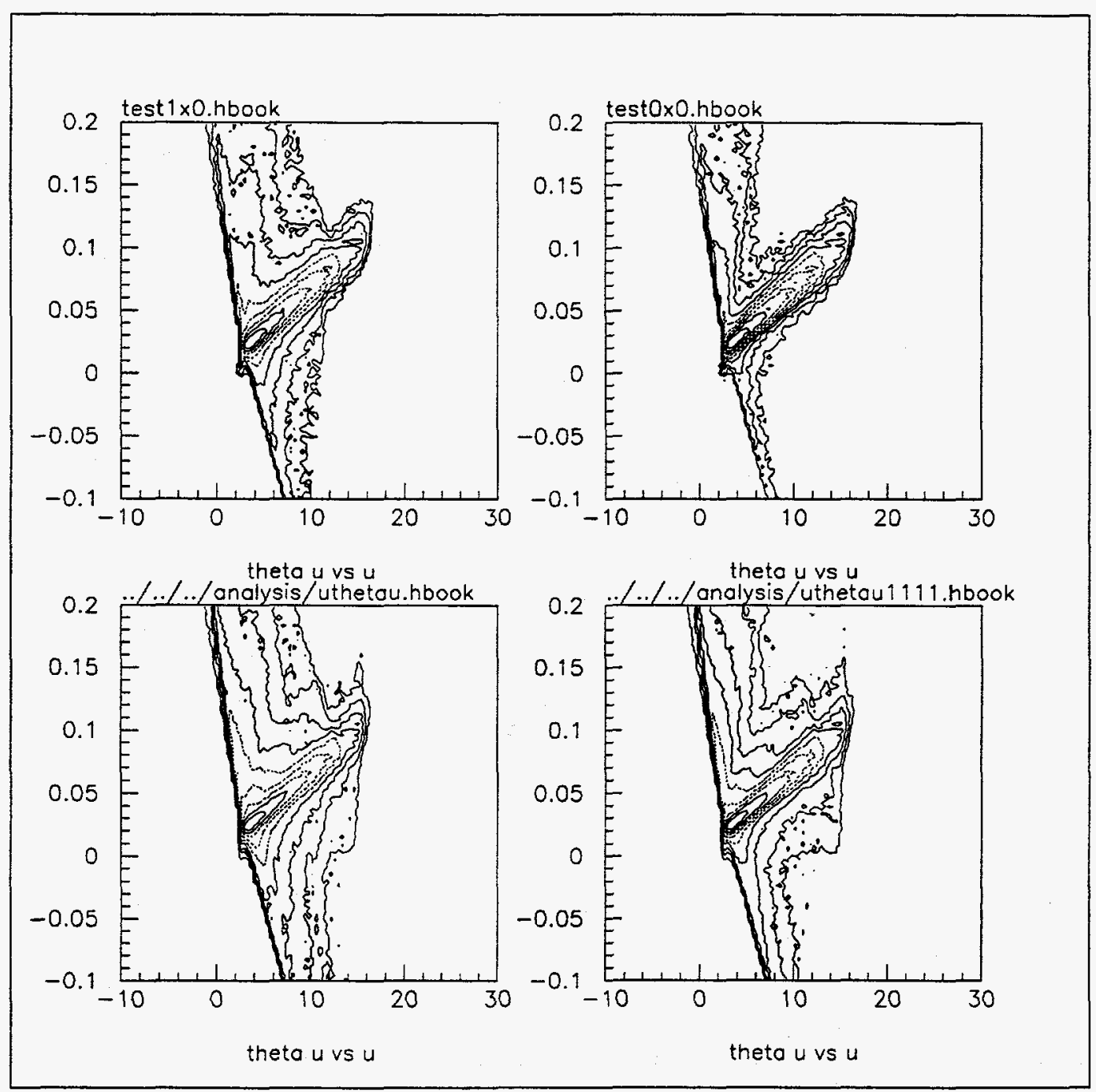

Figure 4.25: Location of tracks at the lead in phase space $\left(u, \theta_{u}\right)$ for lead-in GEANT, lead-out GEANT, lead-in run 1110, lead-out run 1111. 


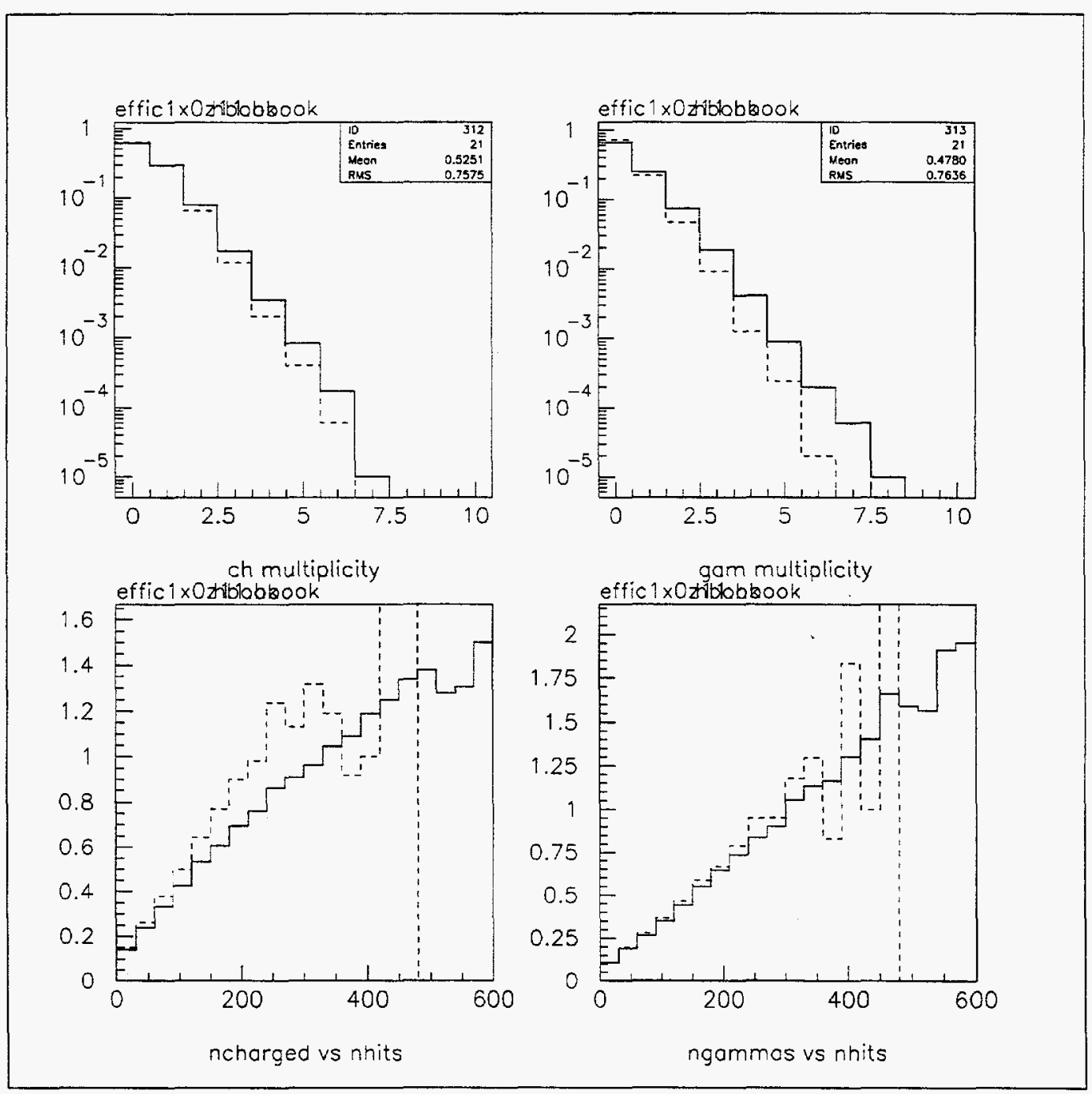

Figure 4.26: PYTHIA charged-track and photon multiplicity distributions, mean multiplicities vs NHITS for all lead-in GEANT events (solid) and for non-vetoed events (dashed). 


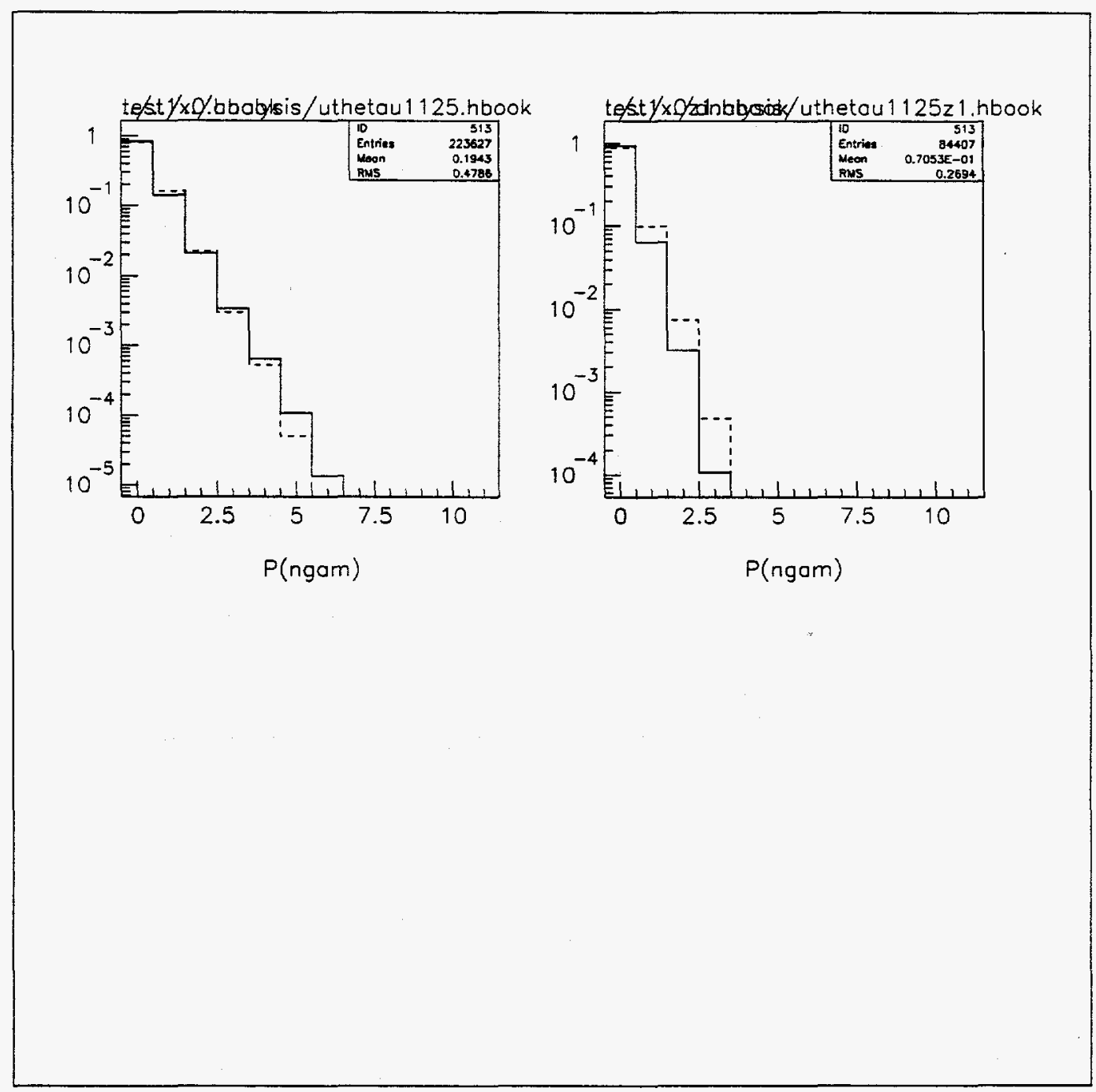

Figure 4.27: Observed photon multiplicity distribution for all events and for non-vetoed events from lead-in run 1125 (solid) and lead-in GEANT (dashed). 


\begin{tabular}{|c|c|c|c|c|}
\hline $\begin{array}{c}\text { track } \\
\text { type }\end{array}$ & $\begin{array}{c}\text { chambers } \\
\text { searched }\end{array}$ & $\begin{array}{c}\text { number of } \\
\text { chambers hit }\end{array}$ & $\begin{array}{c}\text { used-wire } \\
\text { cut }\end{array}$ & $\begin{array}{c}\text { grouping } \\
\text { cut }\end{array}$ \\
\hline charged & $1-24$ & $\geq 22$ & 17 & 16 \\
& & 21 & 17 & 16 \\
\hline photon/ & $9-24$ & $\geq 15$ & 13 & 11 \\
ch tail & & 14 & 13 & 11 \\
\hline mid-8 & $9-16$ & $\geq 7$ & 5 & 6 \\
\hline front-8 & $1-8$ & $\geq 7$ & 5 & 6 \\
\hline
\end{tabular}

Table 4.1: Cuts used by the tracker. 


\section{Chapter 5}

\section{Generating Function Formalism and Robust Observables}

In order to determine the distribution of the neutral fraction $(f)$ of pions in the MiniMax detector, we would like to count the number of charged and neutral pions from a collision entering our acceptance. (Even if only a piece of a DCC domain enters the acceptance, we would expect on average to observe the same $f$ as for the entire domain.) This approach is complicated by many things, including the fact that $\pi^{0}$ 's decay almost immediately into two photons which we identify only if they convert in the lead or scintillator

within the MWPC telescope. To precisely determine the number of $\pi^{0}$ s, we would need to identify both $\gamma$ 's from a decay and reconstruct the pion mass using the electromagnetic calorimeter. 
We are unable to do this due to the fact that the probability of both photons from a decay entering the small acceptance of the calorimeter is only about $15 \%$. However, we have found a set of observables which sample the charged-neutral distribution and are independent of many of the detectorrelated complications and (uncorrelated) efficiencies, and take very different values for pure DCC and for generic particle production. We make many bold assumptions about the production and detection of particles going into the detector in order to establish the robustness of these observables; however, simulations indicate that the assumptions are not unreasonable (see Sec. 6.1.1). We assume that particles other than pions can be ignored, that charged particles and photons are not misidentified, that the production process can be modeled as a two-step process, with the total number of pions given by a parent distribution and the fraction of pions that are neutral given by, e.g., a binomial or DCC distribution function, and that detection efficiencies for finding a charged particle or photon do not depend on the nature of the rest of the event.

These observables and their properties are best understood using generating functions and their factorial moments to describe probability distributions for the production of some species of particle. The generating function formalism has been widely used in multiparticle analysis [51]-[55] and was extended to two variables [56]-[59], [32], e.g., charged and neutral pions by the MiniMax Collaboration in Ref. [60]. The ideas in this section are described 
in detail in that paper.

Some of the difficulties in measuring the charged-neutral distribution are listed below:

1. The MiniMax acceptance is small, so that it is improbable that both $\gamma$ 's from a $\pi^{0}$ enter the detector acceptance;

2. the conversion efficiency per $\gamma$ is only about $50 \%$;

3. not all $\gamma^{\prime}$ s come from $\pi^{0}$ 's;

4. not all charged tracks come from $\pi^{ \pm}$s;

5. because of the small acceptance, the multiplicities are rather low, so that statistical fluctuations are very important;

6. detection efficiencies are not the same for charged tracks and $\gamma$ 's and are momentum-dependent;

7. efficiency functions may depend on the observed multiplicity or other parameters;

8. the efficiency for triggering when no charged track or converted $\gamma$ is produced within the acceptance is relatively low and different from that for events in which at least one charged particle or converted $\gamma$ is detected. 


\subsection{Generating functions for charged-pion- neutral-pion distributions}

If the set of (normalized) probabilities for producing $N$ particles in a given region of phase space is $\{P(N)\}$, then the generating function can be defined as

$$
G(z)=\sum_{N=0}^{\infty} z^{N} P(N)
$$

and contains all the information of the $\{P(N)\}$ :

$$
P(N)=\frac{1}{N !}\left(\frac{d^{N} G}{d z^{N}}\right)_{z=0}
$$

Information can likewise be extracted from the factorial moments, defined as

$$
f_{i} \equiv\left(\frac{d^{i} G(z)}{d z^{i}}\right)_{z=1}=\langle N(N-1) \cdots(N-i+1)\rangle
$$

where $\langle\mathcal{O}\rangle=\sum_{N} \mathcal{O} P(N)$

Now if $p\left(n_{c h}, n_{0}\right)$ is the probability distribution for producing $n_{c h}$ charged and $n_{0}$ neutral pions, the generating function is

$$
G\left(z_{c h}, z_{0}\right)=\sum_{n_{c h}=0}^{\infty} \sum_{n_{0}=0}^{\infty} p\left(n_{c h}, n_{0}\right) z_{c h}^{n_{c h}} z_{0}^{n_{0}}
$$


and the factorial moments for charged $(c h)$ and neutral $(0)$ pions are

$$
f_{i, j}(c h, 0) \equiv\left(\frac{\partial^{i+j} G\left(z_{c h}, z_{0}\right)}{\partial z_{c h}{ }^{i} \partial z_{0}{ }^{j}}\right)_{z_{c h}=z_{0}=1}
$$

We assume that $p\left(n_{c h}, n_{0}\right)$ can be written as the product of a parent distribution $P(N)$ for producing $N$ total pions, and $\hat{p}\left(n_{c h}, n_{0} ; N\right)$, which gives the charged-neutral distribution of pions for a given $N=n_{c h}+n_{0}$ :

$$
p\left(n_{c h}, n_{0}\right)=P(N) \hat{p}\left(n_{c h}, n_{0} ; N\right)
$$

where

$$
\begin{gathered}
\sum_{N=0}^{\infty} P(N)=1 \\
\sum_{n_{c h}=0}^{\infty} \sum_{n_{0}=0}^{\infty} \delta_{N, n_{c h}+n_{0}} \hat{p}\left(n_{c h}, n_{0} ; N\right)=1 .
\end{gathered}
$$

For generic production of pions, the charge is distributed according to a binomial (bin) with mean neutral fraction $\hat{f}=1 / 3$, so that

$$
\hat{p}_{b i n}\left(n_{c h}, n_{0} ; N\right)=\frac{N !}{n_{0} !\left(N-n_{0}\right) !} \hat{f}^{n_{0}}(1-\hat{f})^{n_{c h}}
$$

The corresponding generating function [from Eqs. (5.9), (5.6) and (5.4)] is

$$
G_{b i n}\left(z_{c h}, z_{0} ; \hat{f}\right)=\sum_{N} P(N)\left[\hat{f} z_{0}+(1-\hat{f}) z_{c h}\right]^{N}
$$


Note that $G_{b i n}$ depends only on the linear combination

$$
\zeta \equiv \hat{f} z_{0}+(1-\hat{f}) z_{c h}
$$

Much of the simplicity of the generic case is also realized for what can be called the binomial transform,

$$
\hat{p}\left(n_{c h}, n_{0} ; N\right)=\frac{N !}{n_{0} !\left(N-n_{0}\right) !} \int_{0}^{1} d f p(f) f^{n_{0}}(1-f)^{n_{c h}}
$$

of the arbitrary normalized distribution $p(f)$, such as the DCC distribution $p(f)=1 /(2 \sqrt{f})$. This leads to a wide class of possible pion factorial-moment generating functions, namely

$$
G\left(z_{c h}, z_{0}\right)=\int_{0}^{1} d f p(f) G_{b i n}\left(z_{c h}, z_{0} ; f\right)
$$

where $G_{b i n}\left(z_{c h}, z_{0} ; f\right)$ is given by (5.10) with $\hat{f}$ replaced by an arbitrary $f$, $0 \leq f \leq 1$.

If $P(N)$ is a Poisson distribution, $\left[P(N)=\frac{\mu^{N}}{N !} e^{-\mu}\right.$ with $\left.\mu=\langle N\rangle\right]$ then $G_{b i n}\left(z_{c h}, z_{0} ; \hat{f}\right)=e^{-\mu+\mu \zeta}$, so that $\ln G_{b i n}\left(z_{c h}, z_{0} ; \hat{f}\right)$ is linear in $\zeta$. The PYTHIA simulations yield generating functions that, to good approximation, depend only on a fixed linear combination of $z_{c h}$ and $z_{0}$ (Fig. 5.1); the full detector simulation with GEANT is found to alter this linear behavior slightly. Compare this to the generating function for the DCC distribution which depends 
on both $z_{c h}$ and $z_{0}$ (Fig. 5.2).

\subsection{Generating functions for charged-pion- photon distributions}

Next we take into account the probability $\epsilon_{c h}$ for observing a given primary charged pion in the detector and a probability $\left(1-\epsilon_{c h}\right)$ for not observing it, and the probabilities $\epsilon_{m}, m=0,1,2$, for observing $m$ photons from a $\pi^{0}$ decay, with $\epsilon_{0}+\epsilon_{1}+\epsilon_{2}=1$. We assume that these efficiencies are uncorrelated. Then the generating function for the distribution of observed particles, including efficiencies, is obtained from $G\left(z_{c h}, z_{0}\right)$ [47] by replacing $z_{c h}$ by the generating function

$$
g_{c h}\left(z_{c h}\right)=\left(1-\epsilon_{c h}\right)+\epsilon_{c h} z_{c h}
$$

and $z_{0}$ by the generating function

$$
g_{0}\left(z_{\gamma}\right)=\epsilon_{0}+\epsilon_{1} z_{\gamma}+\epsilon_{2} z_{\gamma}{ }^{2}
$$

For the class of production models characterized by (5.13), this leads to the following factorial-moment generating function for the distribution of observed charged pions and photons:

$$
G_{o b s}\left(z_{c h}, z_{\gamma}\right)=\int_{0}^{1} d f p(f) G_{b i n}\left(g_{c h}\left(z_{c h}\right), g_{0}\left(z_{\gamma}\right) ; f\right)
$$


The charged-pion-photon factorial moments are

$$
f_{i, j} \equiv\left(\frac{\partial^{i, j} G\left(z_{c h}, z_{\gamma}\right)}{\partial z_{c h}{ }^{i} \partial z_{\gamma}{ }^{j}}\right)_{z_{c h}=z_{\gamma}=1}
$$

which introduces the indexing $(i, j)$ with respect to charged particles and photons which will be used in the remainder of this section. The two lowest orders of factorial moments are

$$
\begin{array}{ll}
f_{1,0}=\left\langle n_{c h}\right\rangle & =\langle 1-f\rangle \epsilon_{c h}\langle N\rangle, \\
f_{0,1}=\left\langle n_{\gamma}\right\rangle & =\langle f\rangle\left(\epsilon_{1}+2 \epsilon_{2}\right)\langle N\rangle, \\
f_{2,0}=\left\langle n_{c h}\left(n_{c h}-1\right)\right\rangle & =\left\langle(1-f)^{2}\right\rangle \epsilon_{c h}{ }^{2}\langle N(N-1)\rangle, \\
f_{1,1}=\left\langle n_{c h} n_{\gamma}\right\rangle & =\langle f(1-f)\rangle \epsilon_{c h}\left(\epsilon_{1}+2 \epsilon_{2}\right)\langle N(N-1)\rangle, \\
f_{0,2}=\left\langle n_{\gamma}\left(n_{\gamma}-1\right)\right\rangle & =\left\langle f^{2}\right\rangle\left(\epsilon_{1}+2 \epsilon_{2}\right)^{2}\langle N(N-1)\rangle+\langle f\rangle 2 \epsilon_{2}\langle N\rangle,
\end{array}
$$

where the overall statistical averages for the charged, photon, and chargedphoton factorial moments are expressed, in an obvious notation, in terms of the independent moments taken with respect to the $P(N)$ and $p(f)$ distributions. The second-order factorial moments represent the lowest-order correlative effects among charged pions and photons.

\subsection{Robust observables}

We would like to construct a measure from the moments in the form of a ratio in order to cancel out as many effects as possible, apart from the $p(f)$ 
averages which give information about the charged-neutral distribution. The gamma-gamma correlation $\left(f_{0,2}\right)$ involves a term proportional to $\epsilon_{2}\langle N\rangle$ that cannot be cancelled by any other moments of this order. However, the ratio

$$
r_{1,1}=\frac{f_{1,1} f_{1,0}}{f_{2,0} f_{0,1}}=\frac{\left\langle n_{c h} n_{\gamma}\right\rangle\left\langle n_{c h}\right\rangle}{\left\langle n_{c h}\left(n_{c h}-1\right)\right\rangle\left\langle n_{\gamma}\right\rangle} .
$$

involving the other four moments has complete cancellation of all reference to the background distribution $P(N)$ and the efficiencies $\epsilon_{1}, \epsilon_{2}$, and $\epsilon_{c h}$ for generating functions of the form (5.16):

$$
r_{1,1}=\frac{\langle f(1-f)\rangle\langle(1-f)\rangle}{\left\langle(1-f)^{2}\right\rangle\langle f\rangle} .
$$

For generic pion production, $p(f)=\delta(f-\hat{f})$ and

$$
r_{1,1}(g e n)=1 .
$$

For a DCC distribution, $p(f)=1 /(2 \sqrt{f})$, the ratio is

$$
r_{1,1}(D C C)=\frac{1}{2}
$$

Therefore, the pure DCC and generic distributions should be easily distinguishable if the statistical uncertainties are not too large.

We can simplify the formulas slightly by introducing normalized factorial 
moments. For the production of $N$ particles, these are given by

$$
F_{i} \equiv \frac{\langle N(N-1) \ldots(N-i+1)\rangle}{\langle N\rangle^{i}}
$$

A generalization of the $F_{i}$ 's to normalized moments for charged track and photon production is

$$
F_{i, j}=\frac{\left\langle n_{c h}\left(n_{c h}-1\right) \ldots\left(n_{c h}-i+1\right) n_{\gamma}\left(n_{\gamma}-1\right) \ldots\left(n_{\gamma}-j+1\right)\right\rangle}{\left\langle n_{c h}\right\rangle^{i}\left\langle n_{\gamma}\right\rangle^{j}}
$$

In particular,

$$
F_{i, 0}=\frac{F_{i}\left\langle(1-f)^{i}\right\rangle}{\langle(1-f)\rangle^{i}}
$$

and

$$
F_{i, 1}=\frac{F_{i+1}\left\langle f(1-f)^{i}\right\rangle}{\langle f\rangle\langle(1-f)\rangle^{i}}
$$

where $F_{i}$ refers to the $i$ th normalized factorial moment (5.23) of the $P(N)$ distribution for the total multiplicity.

We see that $r_{1,1}=F_{1,1} / F_{2,0}$. A generalization of $r_{1,1}$ to a family of robust observables is

$$
r_{i, 1}=\frac{F_{i, 1}}{F_{i+1,0}}=\frac{\langle(1-f)\rangle\left\langle f(1-f)^{i}\right\rangle}{\langle f\rangle\left\langle(1-f)^{i+1}\right\rangle},
$$

where again the dependence on the parent distribution and efficiencies has dropped out. For all $i \geq 1$, generic particle production yields

$$
r_{i, 1}(\text { gen })=1
$$


while for DCC,

$$
r_{i, 1}(D C C)=\frac{1}{i+1}
$$

Thus, $r_{i, 1}$ becomes more sensitive to the difference between DCC and generic production mechanisms with increasing order of the moments. This reflects the broadness of the DCC neutral-fraction distribution relative to the binomial distribution of the generic case. If only a fraction of particle production is due to DCC, the signal will be easier to see in the higher-order ratios, which are sensitive to the tail of the charged-photon distribution, where the ratio of DCC to generic production is relatively high. The ratios

$$
r_{i, j}=\frac{F_{i, j}}{F_{i+j, 0}}
$$

involving higher-order gamma correlations $\left(F_{i, j}, j>1\right)$ are not robust because the moments $F_{i, j}$ depend on the photon detection efficiencies. However, the moments can be expressed in terms of only one combination of these efficiencies along with the mean number of photons, namely

$$
\xi=\frac{2 \epsilon_{2}}{\left(\epsilon_{1}+2 \epsilon_{2}\right)\left\langle n_{\gamma}\right\rangle},
$$

as

$$
F_{i, j}=\sum_{m=0}^{[j / 2]} c_{j, m} \xi^{m} F_{i+j-m} \frac{\left\langle(1-f)^{i} f^{j-m}\right\rangle}{\langle(1-f)\rangle^{i}\langle f\rangle^{j-m}}
$$

The coefficients $c_{j, m}$ are obtained from the identity [61], true for any differ- 
entiable function $D\left(z^{2}\right)$,

$$
\frac{d^{j} D\left(z^{2}\right)}{(d z)^{j}}=\sum_{m=0}^{[j / 2]} c_{j, m} 2^{m}(2 z)^{j-2 m} \frac{d^{j-m} D\left(z^{2}\right)}{\left(d z^{2}\right)^{j-m}}
$$

The first few $c_{j, m}$ are

$$
\begin{aligned}
c_{j, 0} & =1, \\
c_{j, 1} & =j(j-1) / 2, \\
c_{j, 2} & =3 j ! / 4 !(j-4) ! .
\end{aligned}
$$

The ratios $r_{i, j}$ can be used in the analysis of experimental distributions, with the understanding that the parameter $\xi$ is to be determined from the data.

Generally, we have the bounds and limiting values

$$
\begin{gathered}
r_{i, j}(\text { gen }) \geq 1, \\
\left.r_{i, j}(\text { gen })\right|_{\xi=0}=1, \\
\left.r_{i, j}(D C C)\right|_{\xi=0}=\frac{i !(2 j-1) ! !}{(i+j) !} .
\end{gathered}
$$

Finally, we turn to the effect of the MiniMax trigger on the moments and ratios. As discussed in Sec. 2.2, events in which no charged particle or converted $\gamma$ goes through the acceptance of the detector are triggered with lower efficiency, $\epsilon$, than other events. Take $p^{\text {obs }}\left(n_{c h}, n_{\gamma}\right)$ to be the (normalized) 
probability for observing an event with $n_{c h}$ charged particles and $n_{\gamma}$ converted $\gamma$ 's in the acceptance assuming perfect triggering, and $p^{\text {trig }}\left(n_{c h}, n_{\gamma}\right)$ to be the probability including the effects of both the trigger and the particle detection efficiencies. An effective model for the effect of the trigger on the probability is given by

$$
\begin{array}{ll}
p^{t r i g}(0,0)=\epsilon \alpha p^{o b s}(0,0), & n_{c h}=n_{\gamma}=0 \\
p^{t r i g}\left(n_{c h}, n_{\gamma}\right)=\alpha p^{o b s}\left(n_{c h}, n_{\gamma}\right), & n_{c h}+n_{\gamma}>0
\end{array}
$$

where $\alpha$ is a normalization factor,

$$
\alpha=\left[1+(1-\epsilon) p^{o b s}(0,0)\right]^{-1}
$$

When we incorporate these trigger efficiencies,

$$
f_{i, j} \rightarrow \alpha f_{i, j}
$$

and

$$
F_{i, j} \rightarrow \alpha^{1-i-j} F_{i, j}
$$

leaving the $r_{i, j}$ robust in the sense that they are also independent of $\epsilon$.

In summary,

1. the $r_{i, j}$ do not depend upon the form of the parent pion multiplicity distribution; 
2. the $r_{i, j}$ are independent of the detection efficiencies for finding charged tracks, provided these efficiencies are not correlated with each other or with other variables such as total multiplicity or background level;

3. the $r_{i, 1}$ are also independent of the $\gamma$ efficiencies in the same sense as above; the $r_{i,(j>1)}$ depend only upon one parameter, $\xi$, which reflects the relative probability of both photons from a $\pi^{0}$ being detected in the same event;

4. in all cases the $r_{i, j}$ are independent of the magnitude of the null trigger efficiency;

5. the ratios $r_{i, j}$ possess definite and very different values for pure generic and pure DCC pion production. 


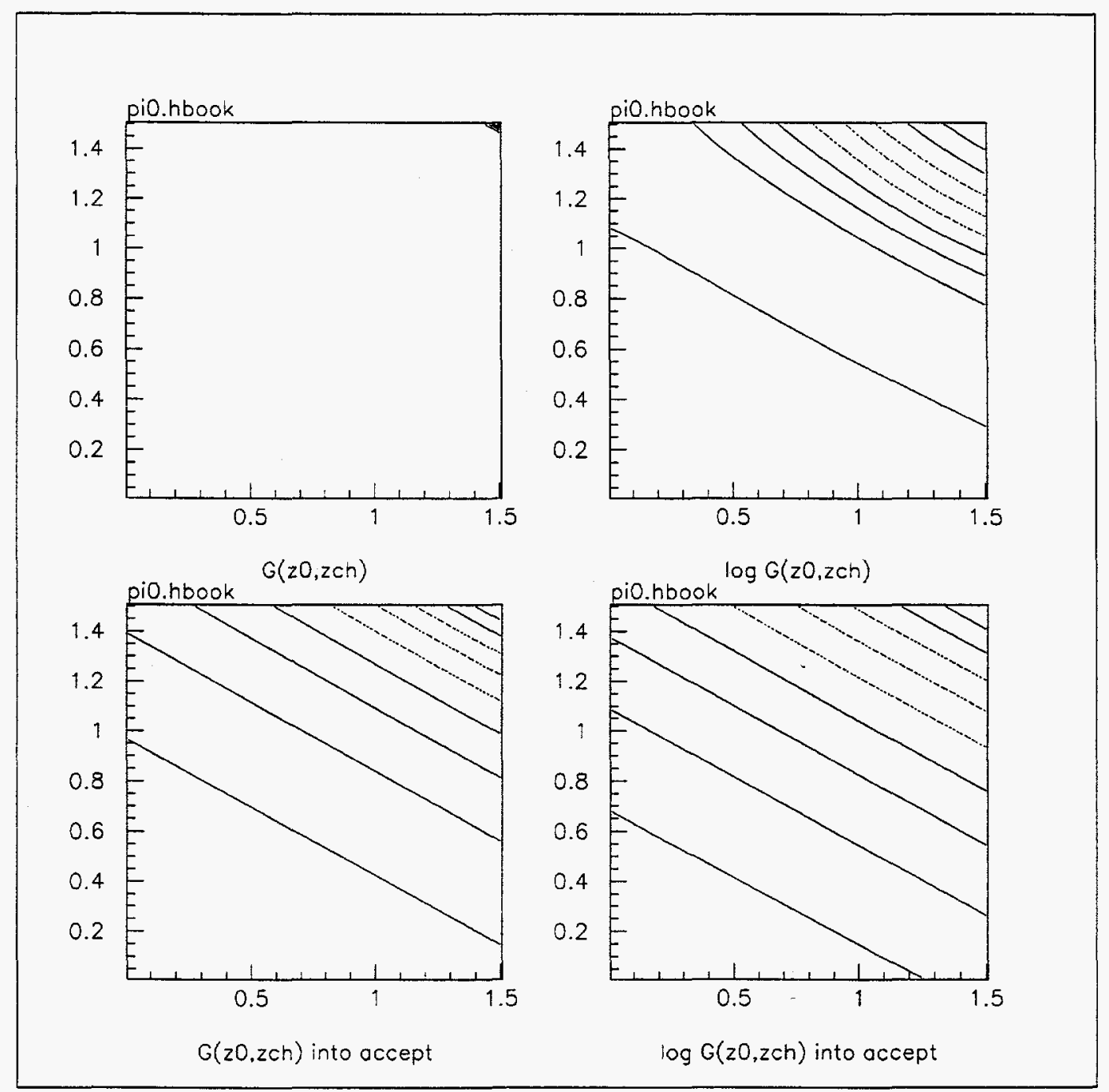

Figure 5.1: Contour plots of the generating function $G\left(z_{c h}, z_{0}\right)$ and $\log G\left(z_{c h}, z_{0}\right)$ from all PYTHIA charged and neutral pions, and from those entering the MiniMax acceptance (the vertical axis is $z_{c h}$ and the horizontal is $z_{0}$ ). 


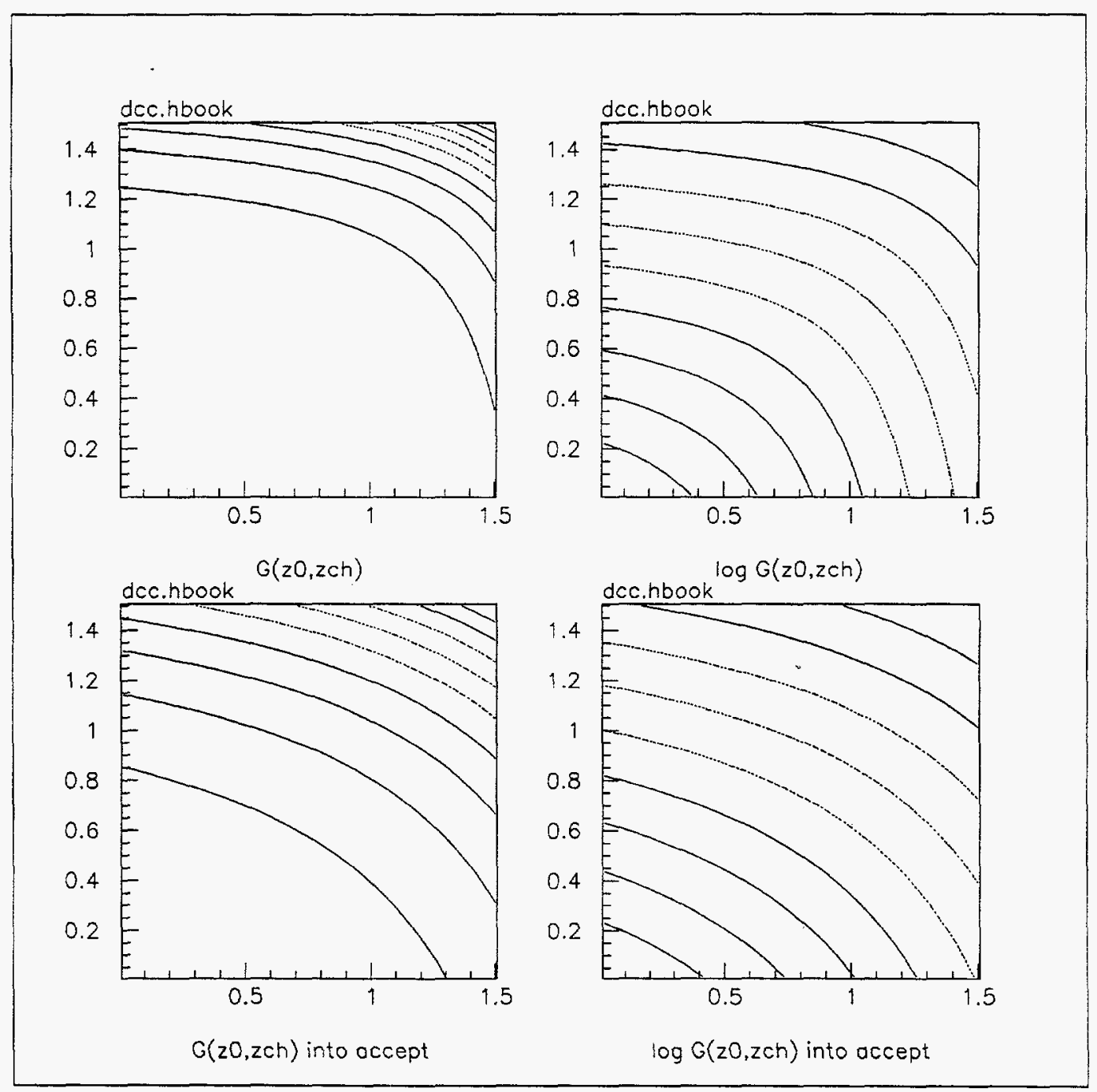

Figure 5.2: Contour plots of the generating function $G\left(z_{c h}, z_{0}\right)$ and $\log G\left(z_{c h}, z_{0}\right)$ from all charged and neutral pions from the DCC generator, and from those entering the MiniMax acceptance (the vertical axis is $z_{c h}$ and the horizontal is $z_{0}$ ). 


\section{Chapter 6}

\section{Results}

\subsection{DCC: Calculated factorial moments and robust observables}

For each real or simulated event, the vertexer returns the number of charged tracks and photons found. Then the frequency distribution for observing a given number of charged and photon-conversion tracks, $\mathcal{N}\left(n_{c h}, n_{\gamma}\right)$, is used to calculate the factorial moments, assuming that the probability of observing an event with a given number of charged tracks and converted photons is given by the observed distribution:

$$
P\left(n_{\mathrm{ch}}, n_{\gamma}\right) \approx \mathcal{N}\left(n_{\mathrm{ch}}, n_{\gamma}\right) / N,
$$


where $N$ is the total number of events

$$
N=\sum_{n_{c h}=0}^{\infty} \sum_{n_{\gamma}=0}^{\infty} \mathcal{N}\left(n_{c h}, n_{\gamma}\right)
$$

Statistical errors are estimated assuming Poisson fluctuations and standard propagation of errors formalism. Appendix A shows the derivation of some of the formulas used to calculate uncertainties in the moments and $r_{i, j}$. In order to check the accuracy of the calculated uncertainty in the $r_{i, j}$, the values of $r_{1,1}$ and $\sigma_{\tau_{1,1}}$ were determined for groups of 25000 events from runs $1096,1099,1103,1109,1110,1125,1126,1127,1137$, and 1139. Then a Gaussian was fit to the histogram of $(r(i)-\langle r\rangle) / \sigma_{r}(i)$, where $r(i)$ and $\sigma_{r}(i)$ are $r_{1,1}$ and $\sigma_{r_{1,1}}$, respectively, for the $i$ th group of events, and $\langle r\rangle=1.0228$ is the mean $r_{1,1}$ for all events. The fitted Gaussian is shown in Fig. 6.1, and has mean $\mu=0.012 \pm 0.144$ and standard deviation $\sigma=0.985 \pm 0.160$. Note that if the calculated uncertainty is equivalent to the actual statistical uncertainty, then the standard deviation of the Gaussian should be $\sigma=1$. Since this is true to within errors, we believe that the calculated uncertainty accurately represents the true statistical uncertainty.

\subsubsection{Simulations}

The moments and $r_{i, j}$ were calculated for approximately $1.5 \times 10^{5}$ PYTHIA events which would be seen by the detector (pass trigger cuts) and $2 \times 10^{4}$ 
pure DCC events, and the results are shown in Table 6.1. The PYTHIA results are given for perfect charged-and photon-finding efficiencies, along with the output of running these events through the GEANT simulation. The DCC events are also processed by the same GEANT simulation, except that the trigger is not used since there are no particles generated in the $\overline{\mathrm{p}}$ direction. For purposes of comparison, the predicted values for idealized binomial and $1 /(2 \sqrt{f})$ distributions are included. [For $j>1$, these depend on the parameter $\xi$, which was estimated using the relationship between $f_{0,2}, f_{2,0}$ assuming a binomial distribution. Then the observed $\left\langle n_{\gamma}\right\rangle$ from PYTHIA/GEANT was used to obtain $2 \epsilon_{2} /\left(\epsilon_{1}+2 \epsilon_{2}\right) \approx 0.08 \pm 0.01$. The $F_{i}$ used in these predictions were also determined from the PYTHIA/GEANT data. We do not expect these values to be correct for the DCC case; in particular, the simulated DCC pions have significantly lower $\left\langle p_{T}\right\rangle$ than the PYTHIA pions, so that the detection efficiencies $\epsilon_{1}$ and $\epsilon_{2}$ are not the same. However, the values from PYTHIA are used here to illustrate the problems which will arise in DCC searches using these non-robust ratios.]

The $r_{i, 1}$ obtained by counting numbers of charged tracks and photons aimed into the acceptance by PYTHIA are within two standard deviations of 1.00. This suggests that something close to a binomial charged-neutral distribution is used by PYTHIA. Detector effects are included by running the events through GEANT. The fact that the lower-order (statistically significant) $r_{i, 1}$ did not differ by more than about $10 \%$ (and are in fact within 
$2 \sigma$ ) of the values from the PYTHIA input justifies the claim that the robust observables are indeed insensitive to detection efficiencies, and also that correlations of efficiencies with multiplicities and momentum do not greatly alter the robustness of these variables. The ratios $r_{i, 1}$ obtained from running the DCC events through GEANT are somewhat higher than the values predicted for the $1 /(2 \sqrt{f})$ distribution; however, they are clearly distinguishable from the values for generic production.

The choice of parameters used in the DCC generator is somewhat optimistic; the DCC domain is aimed directly at the center of the acceptance, and the DCC pions have a rather large $\left\langle p_{T}\right\rangle$. As is shown in Fig. 4.17, very low- $p_{T}$ photons are less likely to convert, and the efficiency for detecting those which do convert is only about $40 \%$ for $p_{T} \lesssim 50 \mathrm{MeV}$. Charged pions with low $p_{T}$ are also more difficult to find because they tend to stop or multiple scatter more in the lead; some scatter so much that the tail is not associated with the head by the vertexer, and may be found as a fake photon conversion. Therefore, we varied the $\left\langle p_{T}\right\rangle$ of the pions in the DCC generator. The resulting moments and $r_{i, j}$ are given in Table 6.2. The parameters in model $\mathrm{A}$ are those mentioned previously. Models $\mathrm{B}$ and $\mathrm{C}$ have lower $\left\langle p_{T}\right\rangle, 50 \mathrm{MeV}$ and $25 \mathrm{MeV}$, respectively. In these models, the ratio of mean energy density of the DCC pions to that of generic pions is not changed $(\psi=1)$, which leads to larger numbers of pions in the domain since $\left\langle N_{\pi}\right\rangle \propto \psi / p_{T}$. In order to keep the mean number of pions constant, we also varied $\psi$ with $p_{T}$ in 
models D and E. For DCC domains with very low- $p_{T}$ pions, the values of the robust observables are closer to what we expect for a binomial distribution, so that distinguishing these domains from generically-produced pions using these observables becomes much more difficult.

Of course, we do not expect to observe events consisting of only a DCC domain aimed into the acceptance. Possible scenarios for mixing DCC and generic multiparticle production are discussed in Ref. [60]. For example, any given event could be due to either DCC or generic production, but not both (exclusive production). Perhaps more realistically, the occurrence of DCC in an event could be independent of the generically-produced pions (independent production), or the amount of DCC production could depend on the amount of generic production (associated production). An example of the latter type is the Baked Alaska model [12], which has the number of DCC pions given by $N_{D C C} \sim N_{g e n}{ }^{3 / 2}$.

In order to study the effect of an admixture of DCC with generic events where the amount of DCC produced is independent of the amount of generic production, we added DCC domains from the DCC-generator/GEANT to various fractions of (random) PYTHIA/GEANT events. The effect on the $r_{i, 1}$ is shown in Table 6.3. (Note that a slightly older version of the vertexer was used for this study, which accounts for the discrepancy in $r_{1,1}$ here with no DCC added and in Table 6.1.) The values for $r_{i, 1}$ fall off faster than linearly with fraction of DCC. For a fraction of 1 , i.e. when a DCC domain 
is added to every event, the ratios are higher than for DCC alone, but are still easily distinguishable from the generic values.

\subsubsection{Characteristics of events from lead-in runs}

Ten of the lead-in runs $(1096,1099,1103,1109,1110,1125,1126,1127$, 1137 , and 1139), totaling about 1.5 million events, were used in the following analysis. The running conditions were very clean (e.g. low luminosity) and therefore the diffractive tags had very little contamination from beam-gas interactions. The frequency distribution of events with given numbers of charged tracks and photons is determined (Table 6.4) and used to calculate the factorial moments and $r_{i, j}$. Table 6.5 gives the values for some of these variables. The mean number of charged tracks found per event is about 0.5 and of converted photons is about 0.2 . The lower-order $r_{i, 1}$ are close to what is expected for a binomial distribution $\left(r_{i, 1}=1\right)$. The values for $r_{1,1}$ and $r_{1,2}$ are within two standard deviations of the PYTHIA results. The higher-order ratios are weighted towards bins of $\mathcal{N}\left(n_{c h}, n_{\gamma}\right)$ which are statistically limited, and therefore the deviations from unity are not very significant. In any case, the ratios are not smaller than one as would be expected for a contribution from DCC. Therefore the events appear to be consistent with production by only generic mechanisms. 


\subsubsection{Characteristics of events with diffractive tags}

From the 10 runs used in Sec. 6.1.2, 21412 events have a ktag from the scintillator which detected showers from interactions of diffractive anti-protons in the kicker magnets. The mean numbers of charged particles and photons are lower for ktag events, as would be expected for diffractive events, where a large fraction of the total energy is carried away by the beam remnant, and the charged-charged and charged-gamma correlations are correspondingly lower. Table 6.5 gives the values of the $r_{i, j}$ for the ktag events.

The upstream hadronic calorimeters at $z \approx-25 \mathrm{~m}$ are used to tag events with diffractive anti-protons with $x_{F} \sim 0.5$ and anti-neutrons. Differences related to isospin exchange in diffractive events might be apparent in comparisons between events with an $\bar{n}$ and those with a $\bar{p}$. Figure 6.2 shows histograms of the ADC readout from the $\overline{\mathrm{n}}$ and $\overline{\mathrm{p}}$ calorimeters. Events with ADC $>400$ (in order to cut out background from products of showers in the magnets) were used to calculate the moments and $r_{i, j}$, given in Table 6.5. The mean number of particles found is higher than that in events with the ktag, but lower than in the total sample of events, and is lower for the tag on leading $\bar{n}$ 's than for $\overline{\mathrm{p}}$ 's with half the beam momentum, consistent with energy conservation.

The $r_{i, j}$ for diffractive-tagged events do not differ by more than two standard deviations from the values for the total sample. Therefore, we conclude that there is no evidence for more DCC production in events with diffractive 
tags, offering no support to the conjecture that Centauros are related to DCC and are diffractive in nature.

\subsubsection{Characteristics of events with a pbar multiplicity $\operatorname{tag}$}

Since DCC may be more often present in events with large multiplicity, we would like to find a measure of the multiplicity independent of that in the small acceptance. The multiplicity in the scintillator on the downstream anti-proton side of the collision at $z=-81$ in ("pbar counters") may be correlated with the total multiplicity, and therefore could be such a measure.

In GEANT, where the number of charged tracks hitting the pbar counters is known, a plot of the total energy deposited in the pbar counters against

the pbar multiplicity (Fig. 6.3) shows the correlation between energy and multiplicity which we expect from minimum-ionizing particles. The pbar multiplicity in the real data can thus be found from the mip peaks in the ADC readout of the counters (Fig. 6.4).

In this way, the pbar multiplicities of events in runs 1099, 1103, 1109, 1125,1126 , and 1127 were determined, and events were grouped in bins of pbar multiplicity, such that each bin contained $10 \%$ of the events. The multiplicity in the pbar counters is indeed correlated with that in the acceptance. Table 6.6 shows an increase in $\left\langle n_{c h}\right\rangle$ and $\left\langle n_{\gamma}\right\rangle$ with increasing pbar multiplicity. However, the $r_{i, 1}$ do not appear to vary with multiplicity, and show 
no sign of an increased presence of DCC for higher multiplicity events. The $r_{1,1}$ 's are consistent with each other and with that for all events.

\subsection{Multiparticle analysis}

Plots of $d N_{c h} / d \eta$ and $d N_{\gamma} / d \eta$, uncorrected for detection and trigger efficiencies, are shown in Fig. 6.5 and Fig. 6.6, respectively. The solid line is the result from approximately $1.5 \times 10^{6}$ events from the 10 runs used in previous analysis. The dotted line is the result from the PYTHIA/GEANT simulation, and the dashed line is from the PYTHIA events which passed the GEANT trigger (about $1.5 \times 10^{5}$ events). The charged multiplicity found by the vertexer for GEANT events is higher than that from the PYTHIA input by about 0.15 , or $3 \%$. The number of photons found for GEANT is about $42 \%$ of the PYTHIA input. The charged multiplicity from the data is lower than that from GEANT and from PYTHIA (about $90 \%$ of the GEANT value). The photon multiplicity is approximately $92 \%$ of that in GEANT.

Since multiplicity is correlated with NHITS, and the contribution to the NHITS from background is greater in the real data than in GEANT, it is not unlikely that the cut of NHITS $<600$ would cut more high-multiplicity events from the data sample than from the GEANT. This conjecture is supported by the results in Sec. 4.3.5, which showed a correlation between pipe shower and multiplicity. However, we do not necessarily expect the data to agree with the simulations, since the PYTHIA input is not based on real measurements 
at the cm energy and $\eta$ range of the MiniMax experiment.

\subsection{Low $p_{T}$ photons}

There has been controversial evidence suggesting that the number of low- $p_{T}$ photons produced is larger than what is expected from hadronic decays and QED inner bremsstrahlung, in particular, that there is an excess of photons with $p_{T} \lesssim 20 \mathrm{MeV}$ [62]. We have attempted to study this by observing photon conversions in the $1 / 4$ in $\left(0.07 \mathrm{X}_{0}\right)$-thick $\mathrm{Al}$ window at $z=120 \mathrm{in}$, and measuring the momentum of the conversion products either by their bending in the scintillator $\left(0.03 \mathrm{X}_{0}\right.$-thick at $z=157$ in $)$ due to multiple scattering or by tracking them into the calorimeter and determining the energy deposited in the hit cells. The lead-out runs were used for this so that the conversion tracks would not stop or scatter and lose energy in the lead. Conversions in the window, rather than in the scintillator, for example, were chosen because the increased resolution in $v$ in the front chambers was desirable for determining the momentum of the converted photon.

First, it was necessary to determine which tracks were due to window conversions. To do this, we plotted the $z$ of closest approach between pairs of tracks. The GEANT lead-out data shows a wide peak at the collision point, and a smaller peak at the window, $z \approx 120$ in, as can be seen in Fig. 6.7. For the real data, the peak at the collision point and at the window are fairly clear, but there is also a smaller peak at intermediate $z$. This is 
apparently due to fake tracks made from $u$ wires borrowed from several real tracks, such that the resulting fakes tended to vertex with the real tracks at a location in $z$ between the collision point and the first chamber (which is just behind the window). These fake vertices are removed by requiring the heads to point such that they will hit the lead, and that they hit all eight of the front chambers. The distribution of the $z$ of closest approach for remaining tracks is shown in Fig. 6.8.

A window conversion is defined as a pair of tracks with a $z$ at closest approach between 115 and $150 \mathrm{in}$, and with a reduced $\chi^{2}$ of the match between the tracks at the window (done in the same way as the usual vertexing at the lead) of $\chi^{2} / 2<2.5$ (see Fig. 6.7 for the GEANT distribution and Fig. 6.8 for that of the data). Also, in order to be able to determine the momentum of the conversion tracks by bending in the scintillator or by following them into the calorimeter, each head is required to have exactly one tail which matched to the head with $\chi^{2} / 2<5$.

Both conversion tracks are found by the tracker in about $26 \%$ of the window conversions in GEANT which send both tracks into the acceptance. Only one track is recorded $70 \%$ of the time, usually because the tracks are so close together that they are grouped by the tracker into a single track. This is apparent in Fig. 6.9, which shows the opening angle of window conversion tracks in GEANT and of the pairs of tracks found as window conversions. Conversions with smaller opening angles are much less likely to be found. 
Of the pairs of tracks found as window conversions, approximately $90 \%$ have one tail matched to each head. As is also shown in Fig. 6.9, the efficiency for finding vertices from the low- $p_{T}$ photons that convert in the window is fairly high (because such conversions tend to have larger opening angles). Unfortunately, the low- $p_{T}$ conversion tracks scatter more in the scintillator than high energy particles, so that the efficiency for matching the tails to the heads is relatively low, preventing a determination of the momentum of these tracks.

The momentum of the conversion tracks is determined from the angles between the heads and tails at the scintillator using the relationship between the momentum of a particle and the bending angle of the track due to multiple scattering in a material [36]

$$
\theta_{0} \approx \frac{13.6 \mathrm{MeV}}{\beta c p} z \sqrt{\frac{x}{\mathrm{x}_{0}}},
$$

where $x / \mathrm{X}_{0}$ is the thickness of the material in radiation lengths, $p$ is the momentum and $\beta c$ the velocity of the particle, $z$ is its charged number, and $\theta_{0}$ is the bending angle in the plane of the incident and bent track. The momentum of the photons which converted in the window is taken to be the sum of the momenta of the two conversion tracks. The $p_{T}$ is determined from the total momentum and the angle of the trajectory of the photon, which must have traveled from the collision point to the vertex of the conversion tracks. For GEANT conversions, where the photon momentum is known, the 
$p_{T}$ as determined by bending in the scintillator tends to be lower than the actual $p_{T}$ (Fig. 6.10). Other attempts to find a measure of the momentum, using, for example, the opening angle, were unsuccessful.

Following the conversion tracks into the calorimeter, their energy is determined to be simply the energy deposited in the cell(s) into which the tracks are aimed, without using a clustering algorithm. The $p_{t}$ found with this method is surprisingly accurate (Fig. 6.10). Unfortunately, the actual calorimeter was never successfully calibrated, and therefore this method can not be reliably used on the data.

The $p_{T}$ distributions of "photons" from pairs of tracks which are found as window conversions in the data are shown in Fig. 6.11, although these distributions are not believed to be very accurate. Instead of comparing $p_{T}$ distributions from GEANT and from the data, a comparison of the number of window conversions integrated over all $p_{T}$ was used to look for evidence for an excess of low- $p_{T}$ photons.

First, the origins of pairs of tracks found as GEANT window conversions are determined. The relative contribution from sources other than actual window conversions should be low, and hopefully from sources of similar magnitude in the GEANT and in the data. In a sample of about $10^{5}$ GEANT events, 225 pairs of tracks are identified as window conversions. About half of these really are window conversions (106 conversions in the window and 4 conversions in the first chamber at $z=123 \mathrm{in}$ ). The next largest contribution 
is $19 \%$ from pairs of charged tracks from the collision point which are close together at the window, including 13 dalitz pairs. The large uncertainty in the point of closest approach due to the nearly identical slope of such tracks allows the possibility of their appearing to vertex at the window. Strong interactions in the window, neutral particle decays (e.g. $K_{s}^{0}, \Lambda^{0}$ ), pairs of a primary charged track from the collision and a secondary charged track, and pairs of a charged track and a fake each contribute between 5 and $7 \%$ of the pairs found as window conversions. Five events have conversions in the window of secondary photons. The remaining few events involve pairs of secondary tracks from interactions in other material.

Almost $10^{6}$ events from lead-out runs $(1089,1093,1104,1108,1111,1123$, $1124,1129,1132)$ were used to search for window conversions in the data. The ratio of the number of window conversions found in the data to that in GEANT is $2121 / 339=6.3$. The ratio of events in the data sample to that in the GEANT sample is $923238 / 155737=5.9$, so that there appears to be an excess of window conversion in the data of about $6 \%$. The uncertainties involved in this analysis do not allow a precise statement either supporting or contradicting the claim of Ref. [62]. 


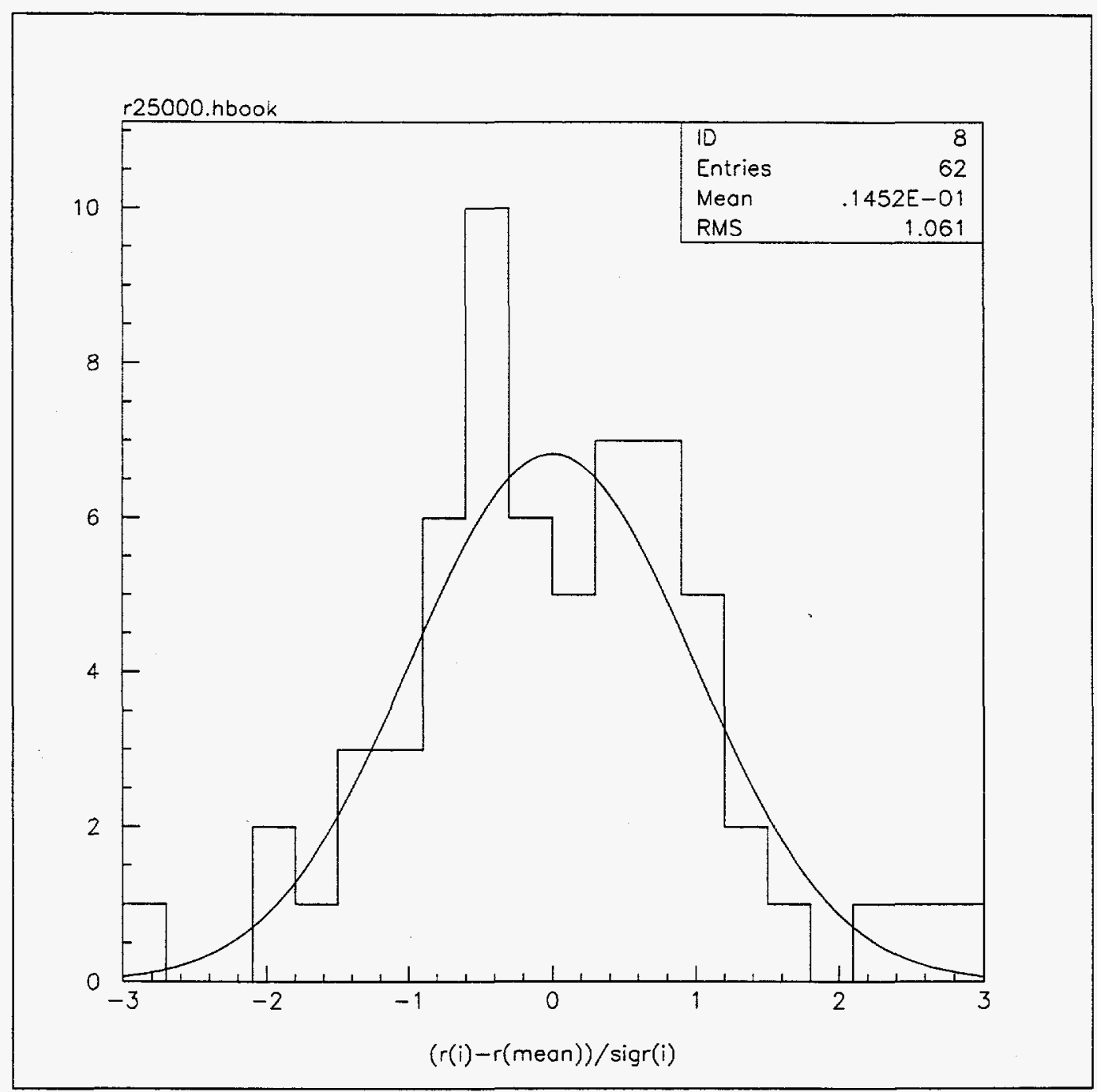

Figure 6.1: Histogram of $(r(i)-\langle r\rangle) / \sigma_{r}(i)$ for groups $(i)$ of 25000 events and Gaussian fit. 


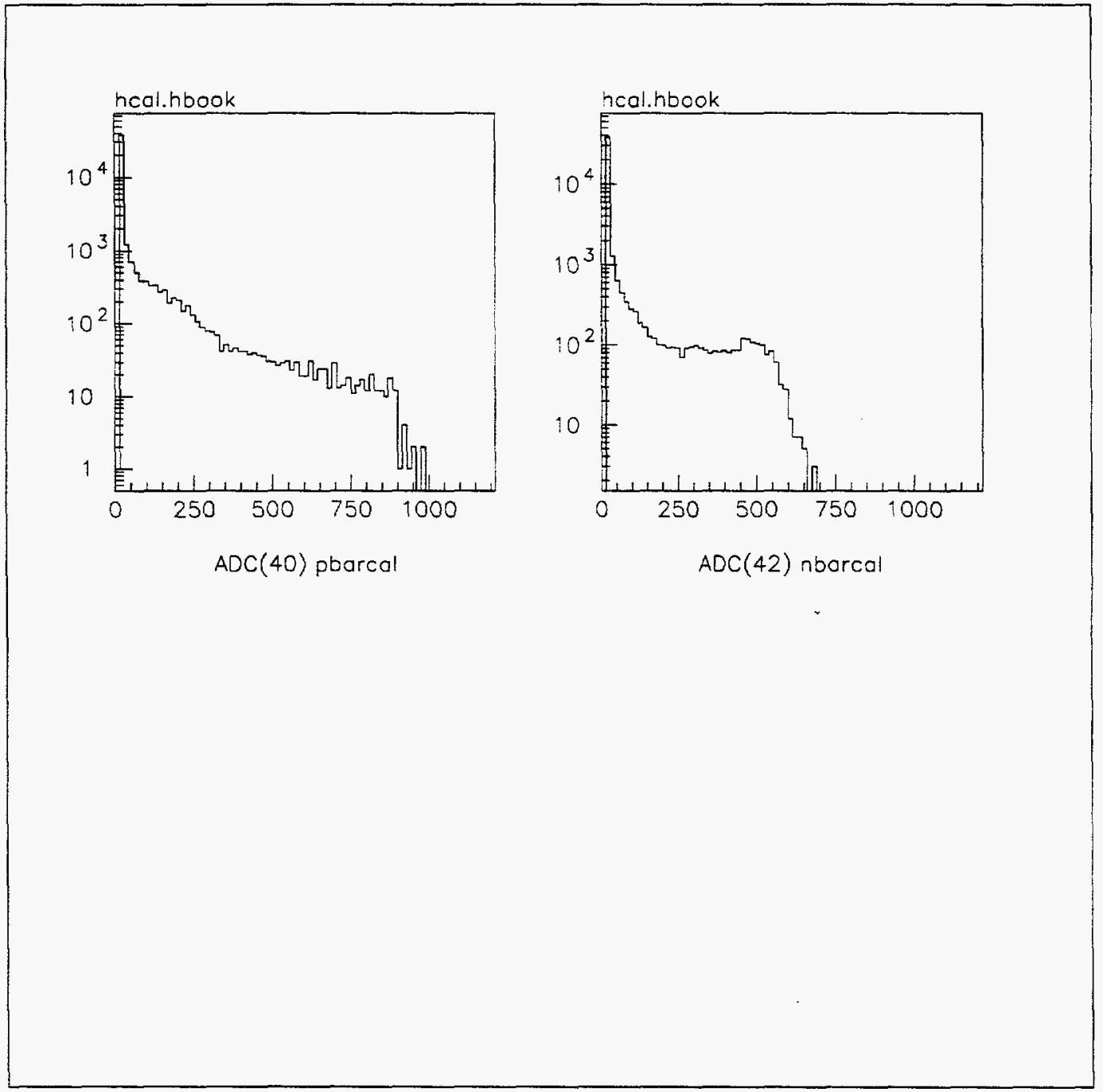

Figure 6.2: $A D C$ values of the hadronic calorimeters which see anti-neutrons $[\mathrm{ADC}(40)]$ and anti-protons $[\mathrm{ADC}(42)]$. 


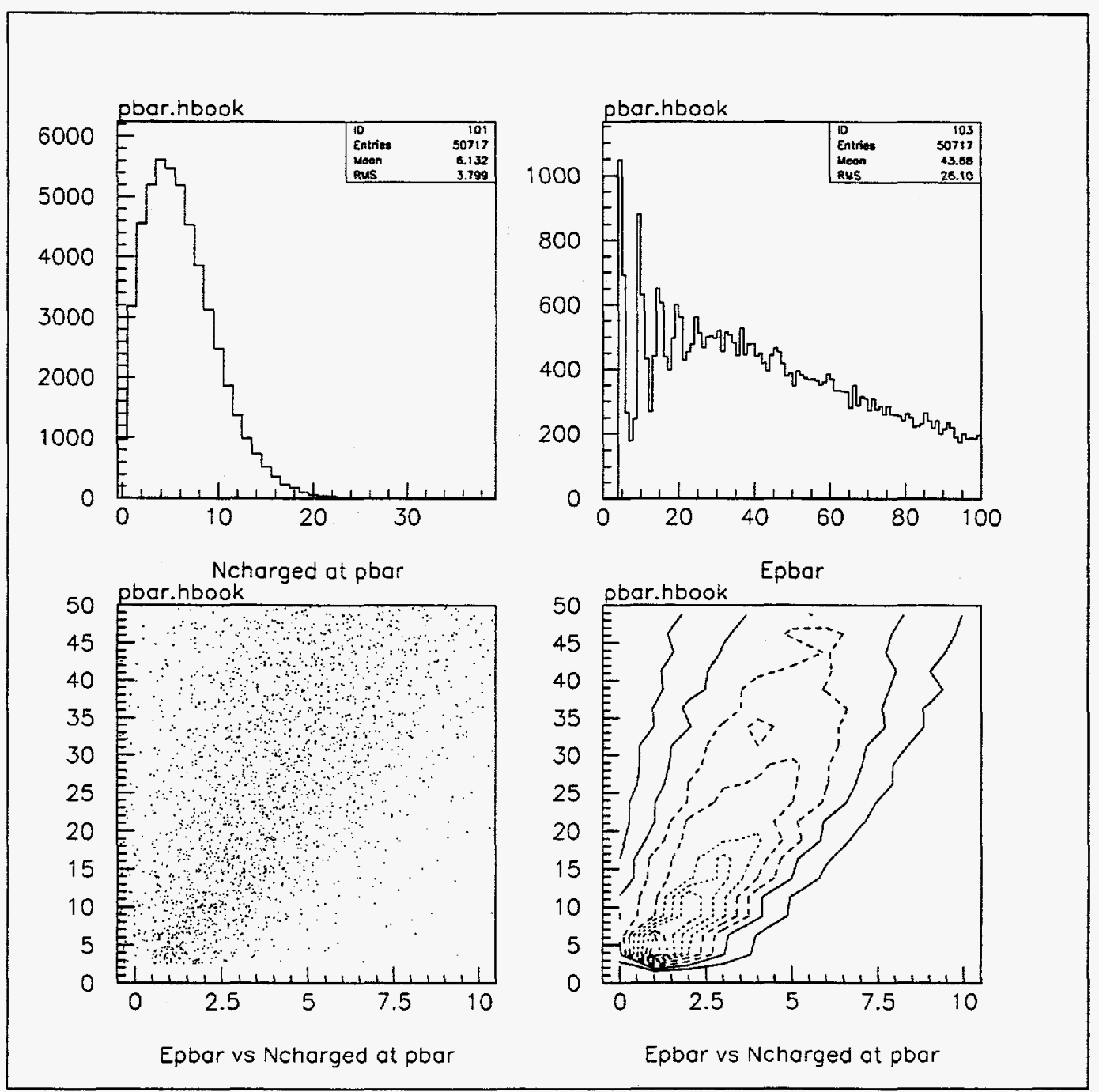

Figure 6.3: Energy (in $\mathrm{MeV}$ ) deposited in the pbar counters vs number of charged particles hitting these counters 


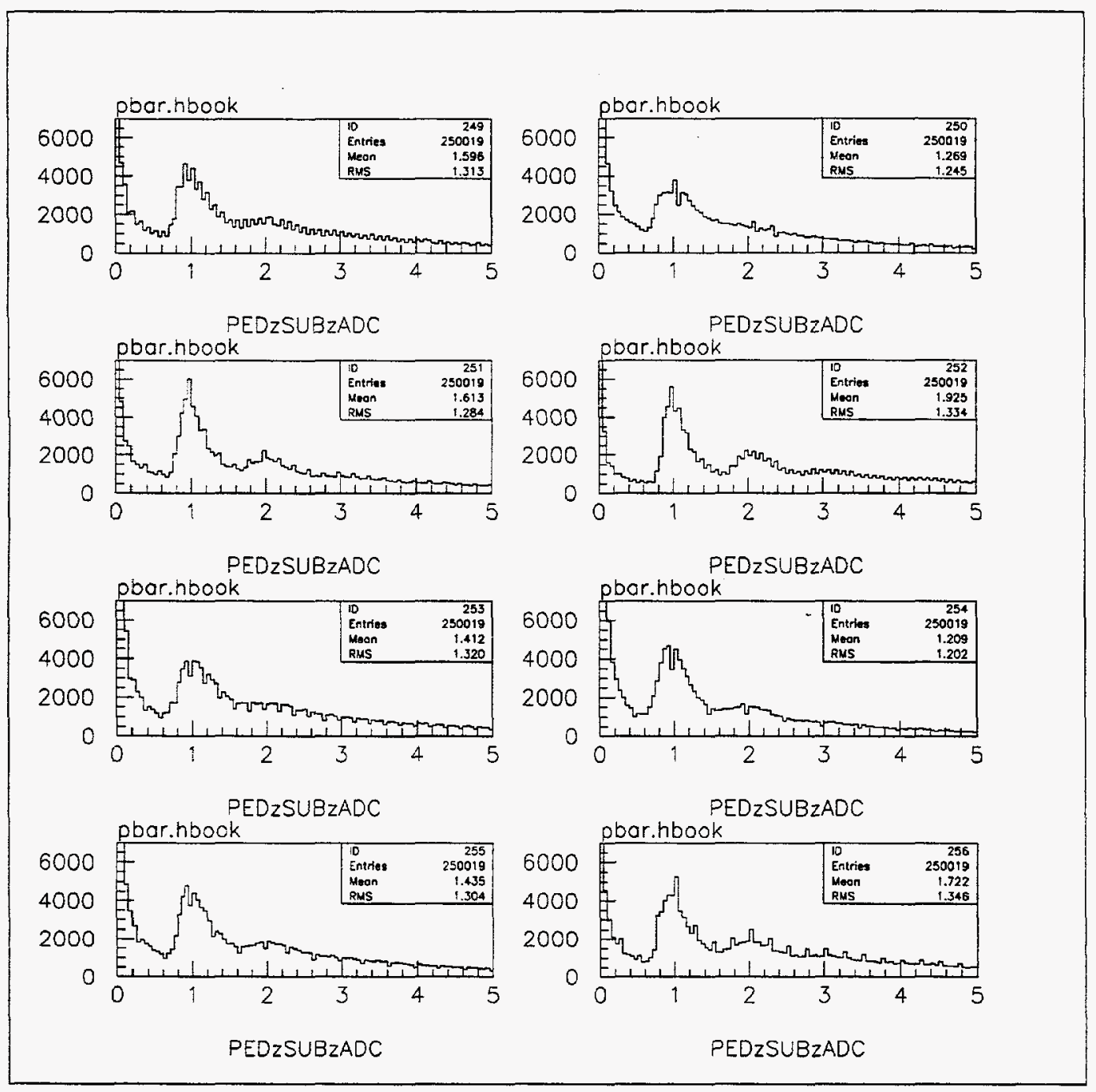

Figure 6.4: PED_SUB_ADC values (in units of mip energies or, equivalently, number of charged particles) of the pbar counters 


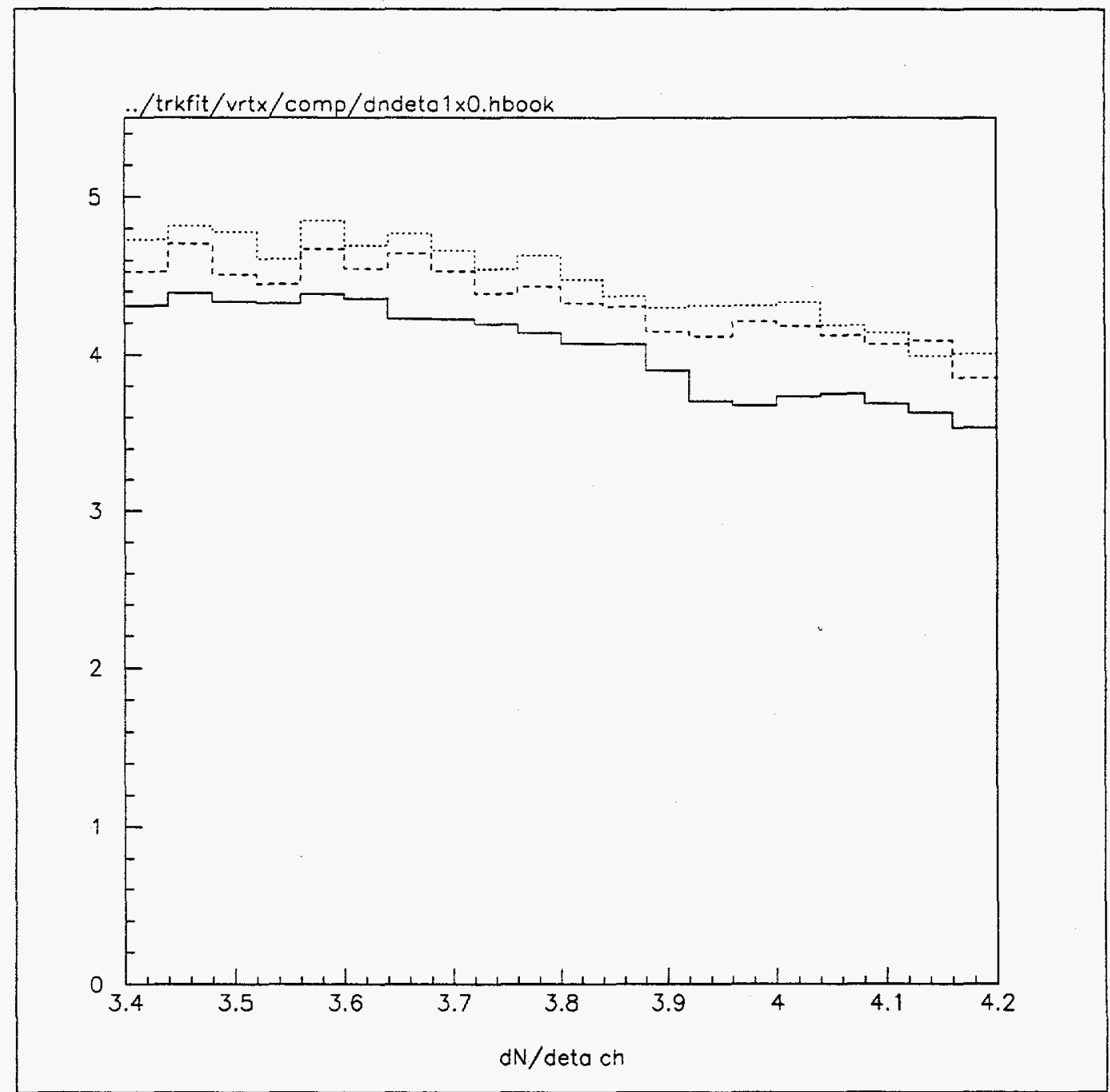

Figure 6.5: Pseudorapidity distribution $d N / d \eta$ vs $\eta$ for charged tracks in the data (solid), GEANT (dotted), and PYTHIA (dashed), uncorrected for efficiencies. 


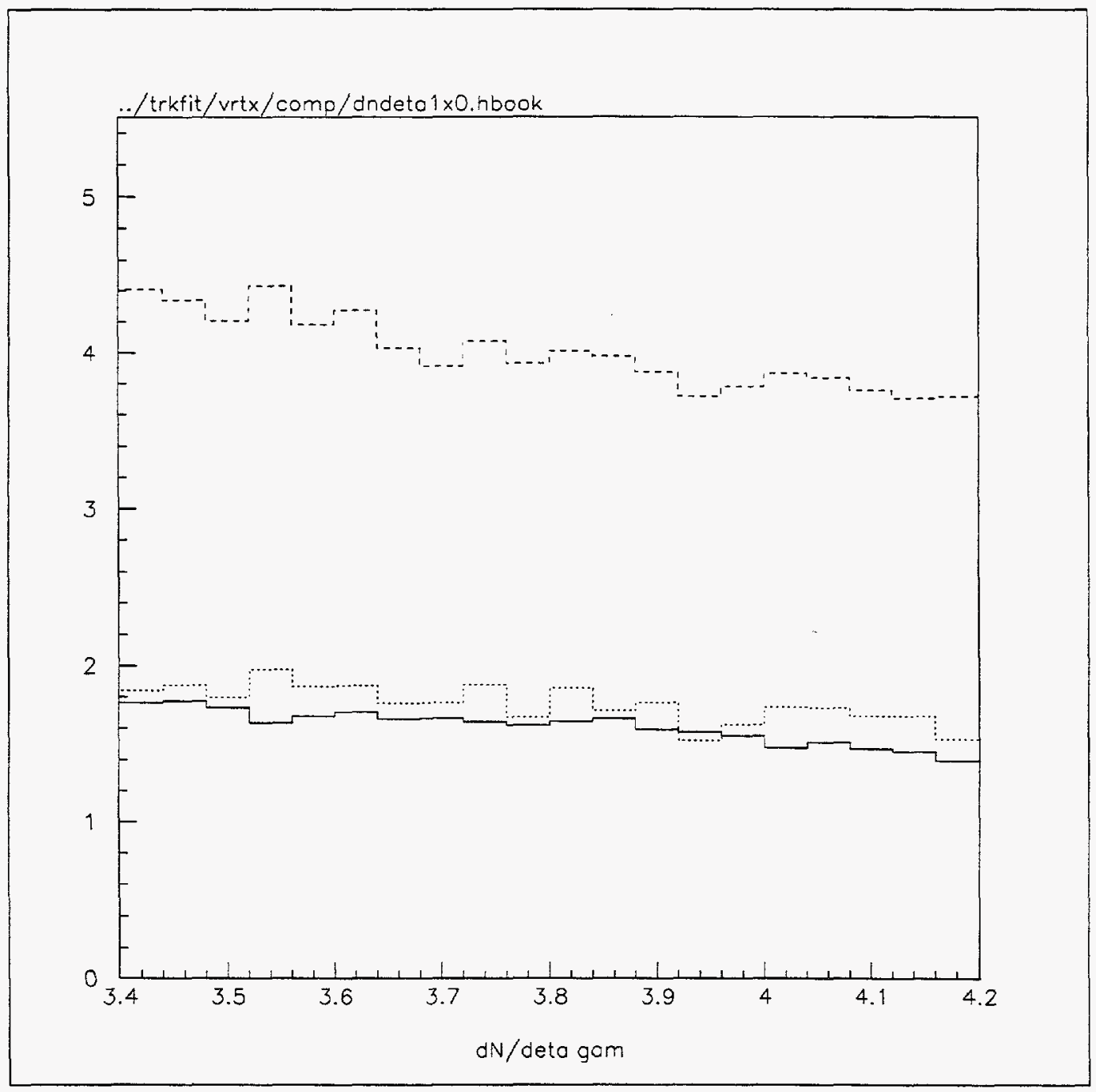

Figure 6.6: Pseudorapidity distribution $d N / d \eta$ vs $\eta$ for photons in the data (solid), GEANT (dotted), and PYTHIA (dashed), uncorrected for efficiencies. 


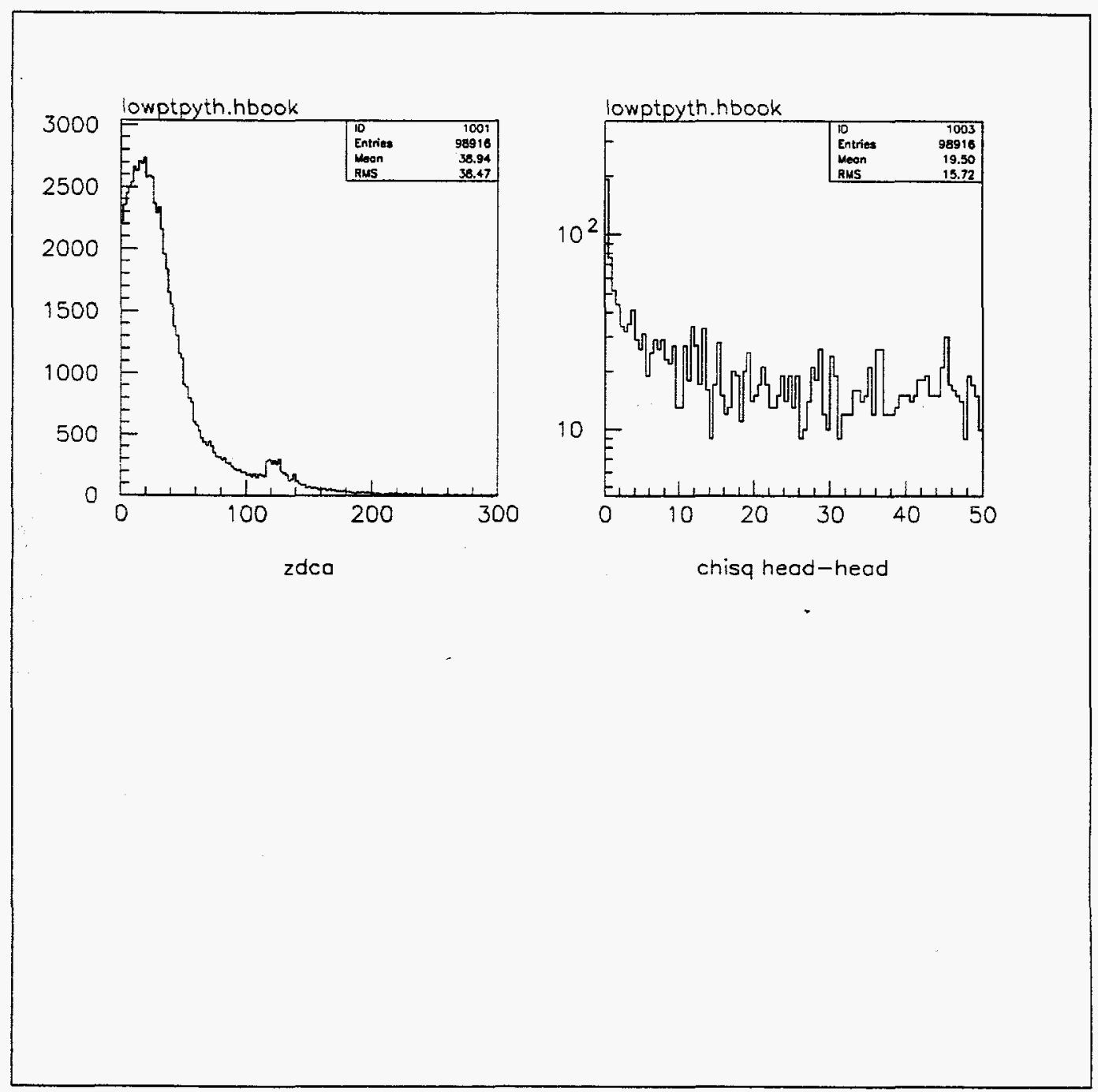

Figure 6.7: Histogram of the $z$ of closest approach between all heads, and of the $\chi^{2}$ of the matching of those pairs in GEANT. 


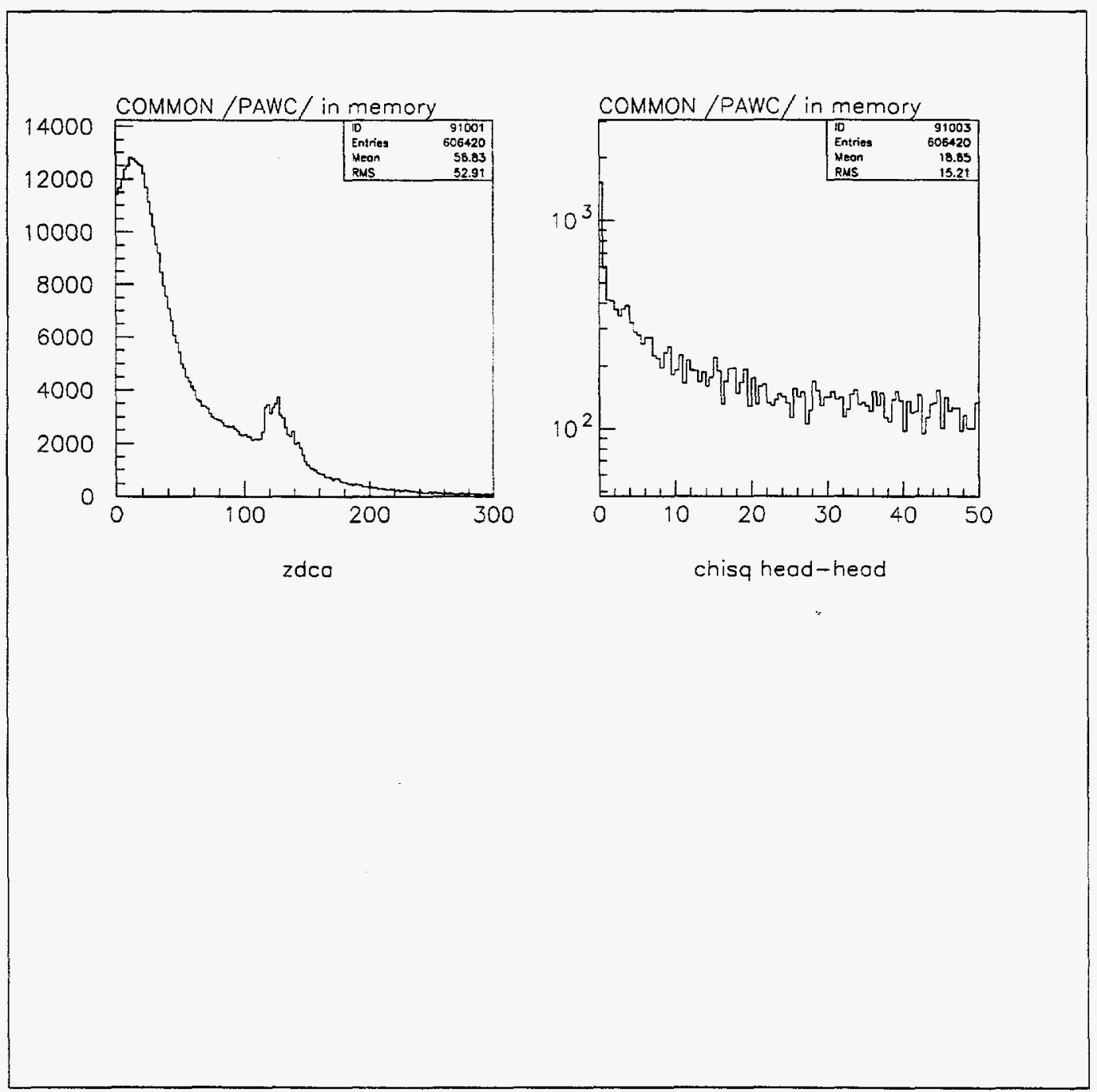

Figure 6.8: Histogram of the $z$ of closest approach between all heads, and of the $\chi^{2}$ of the matching of those pairs in the data. 


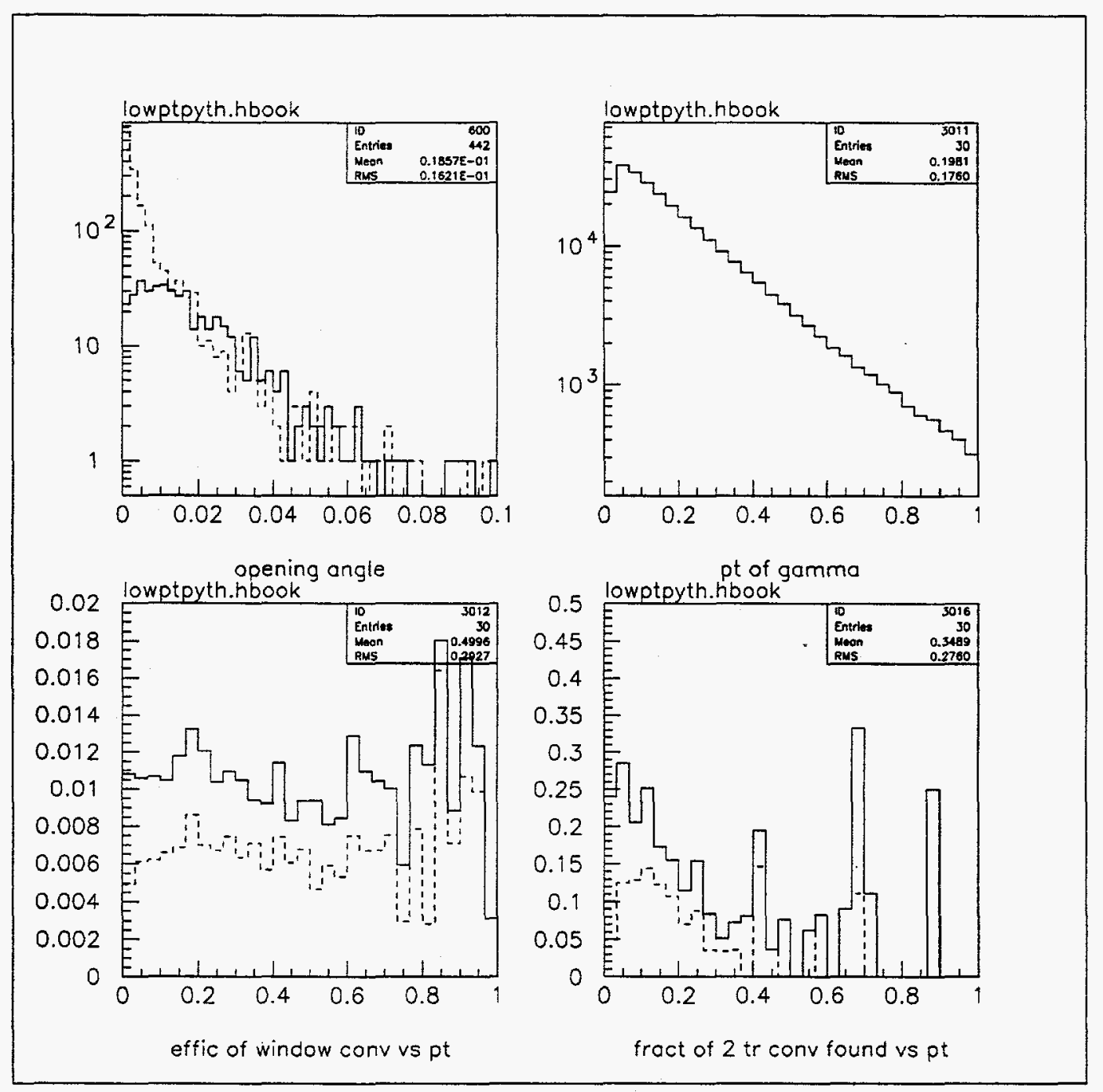

Figure 6.9: Opening angle between pairs found as window conversion tracks in GEANT (solid) and between all GEANT conversion tracks (dashed), $p_{T}$ of all primary photons in GEANT, and efficiencies for finding window conversions as a function of $p_{T}$. In the lower-left plot, the solid line represents the fraction of primary photons which converted in the window, the dashed line is the fraction which also produced two conversion tracks in the acceptance. In the lower right plot, the solid line is the fraction of window conversions with both tracks in the acceptance which were found as a pair of heads, and the dashed line is the fraction which also had one tail per head. 


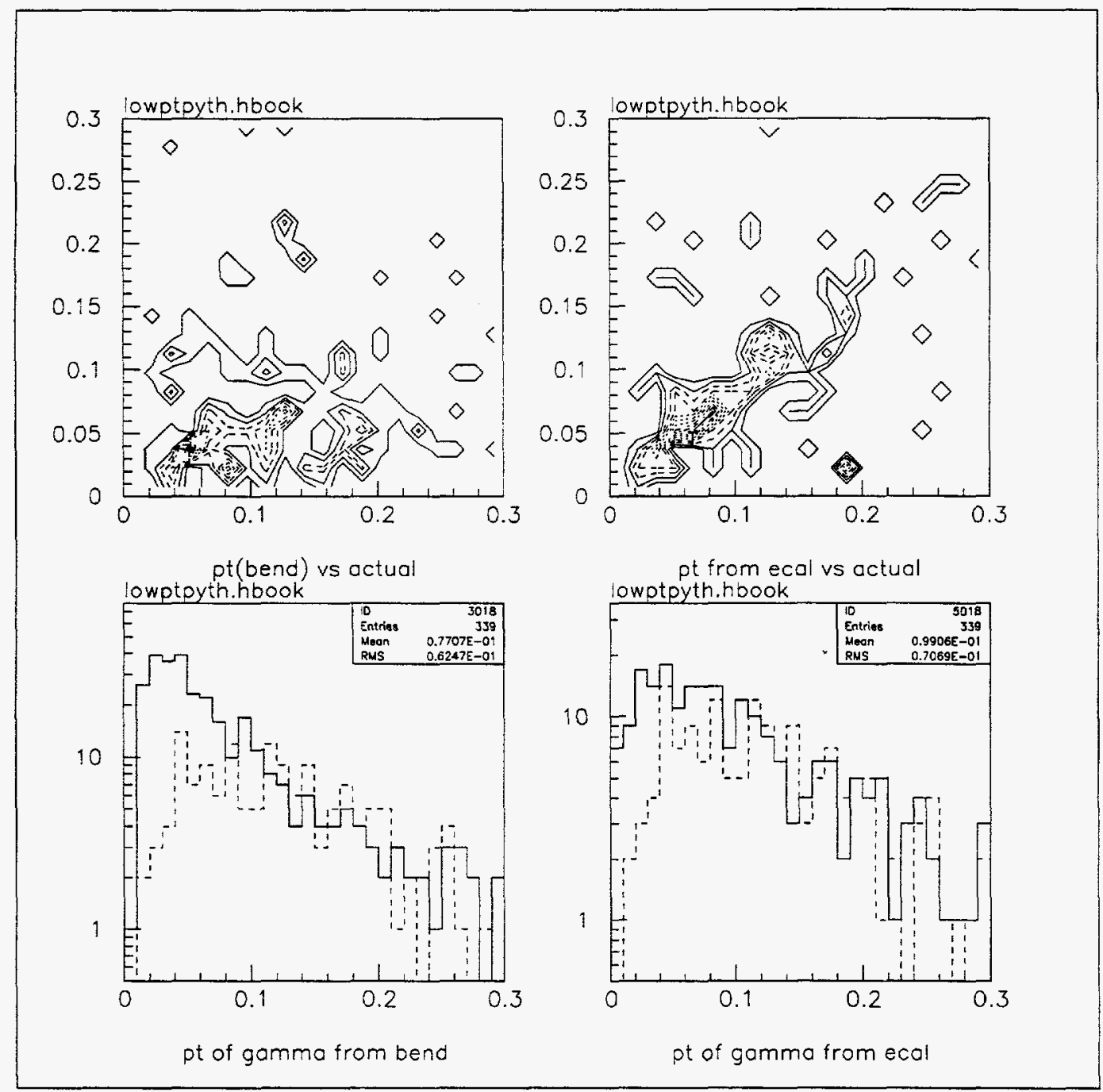

Figure 6.10: Contour plots of the $p_{T}$ found by bending in the scintillator and by using the calorimeter vs the actual $p_{T}$ of the converted photon in GEANT, and corresponding $p_{T}$ distributions (solid is calculated $p_{T}$, dashed is actual). 


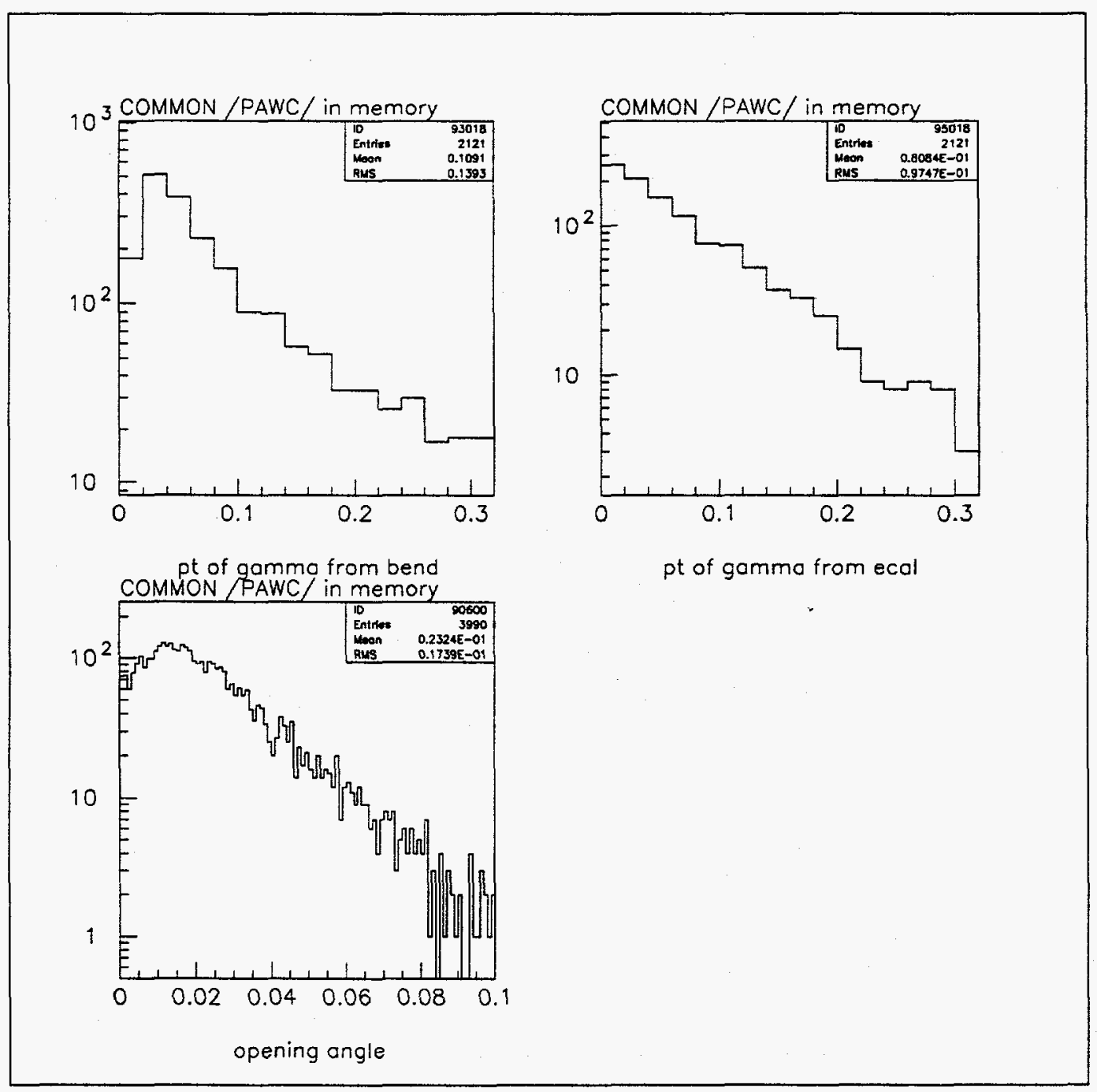

Figure 6.11: Distributions of $p_{T}$ found by bending in the scintillator and by using the calorimeter for converted photons in the data. 


\begin{tabular}{|cc|c|c|c|c|c|}
\hline & & PYTHIA & PYTHIA/GEANT & DCC/GEANT & binomial & $1 /(2 \sqrt{f})$ \\
$i$ & $j$ & $r_{i j} \pm \sigma_{r_{i j}}$ & $r_{i j} \pm \sigma_{r_{i j}}$ & $r_{i j} \pm \sigma_{r_{i j}}$ & $r_{i j}$ & $r_{i j}$ \\
\hline 1 & 1 & $1.00 \pm 0.01$ & $1.00 \pm 0.01$ & $0.58 \pm 0.01$ & 1.00 & 0.50 \\
2 & 1 & $0.99 \pm 0.02$ & $1.00 \pm 0.03$ & $0.44 \pm 0.03$ & 1.00 & 0.33 \\
3 & 1 & $0.96 \pm 0.06$ & $1.03 \pm 0.07$ & $0.39 \pm 0.05$ & 1.00 & 0.25 \\
4 & 1 & $1.02 \pm 0.11$ & $1.29 \pm 0.18$ & $0.42 \pm 0.11$ & 1.00 & 0.20 \\
5 & 1 & $1.32 \pm 0.20$ & $2.03 \pm 0.56$ & $0.51 \pm 0.37$ & 1.00 & 0.17 \\
6 & 1 & $2.06 \pm 2.52$ & $3.61 \pm 1.35$ & $0.51 \pm 0.73$ & 1.00 & 0.14 \\
\hline 0 & 2 & $1.24 \pm 0.01$ & $1.36 \pm 0.02$ & $1.51 \pm 0.05$ & 1.36 & 1.80 \\
1 & 2 & $1.20 \pm 0.04$ & $1.37 \pm 0.05$ & $0.67 \pm 0.05$ & 1.30 & 0.62 \\
2 & 2 & $1.13 \pm 0.09$ & $1.45 \pm 0.15$ & $0.43 \pm 0.08$ & 1.25 & 0.31 \\
0 & 3 & $1.60 \pm 0.06$ & $2.35 \pm 0.15$ & $2.77 \pm 0.34$ & 1.89 & 3.54 \\
1 & 3 & $1.50 \pm 0.15$ & $2.47 \pm 0.30$ & $1.17 \pm 0.27$ & 1.74 & 0.90 \\
0 & 4 & $2.09 \pm 0.24$ & $4.82 \pm 0.86$ & $6.04 \pm 1.84$ & 2.70 & 7.34 \\
\hline
\end{tabular}

Table 6.1: Robust observables $r_{i, j}$ for generic events simulated by PYTHIA and pure-DCC events simulated by the DCC generator, along with predictions for binomial and $1 /(2 \sqrt{f})$ distributions. 


\begin{tabular}{|c|c|c|c|c|c|}
\hline model & $\mathrm{A}$ & $\mathrm{B}$ & $\mathrm{C}$ & $\mathrm{D}$ & $\mathrm{E}$ \\
\hline \# events & 20000 & 10000 & 10000 & 10000 & 10000 \\
\hline$\left\langle p_{T}\right\rangle(\mathrm{MeV})$ & 100 & 50 & 25 & 50 & 25 \\
$\psi$ & 1 & 1 & 1 & 0.5 & 0.25 \\
$\left\langle N_{\pi}\right\rangle$ & 5.0 & 10.0 & 19.9 & 5.0 & 5.0 \\
\hline$\left\langle n_{\text {ch }}\right\rangle$ & $0.97 \pm 0.01$ & $1.96 \pm 0.02$ & $2.98 \pm 0.02$ & $0.99 \pm 0.01$ & $0.93 \pm 0.01$ \\
$\left\langle n_{\gamma}\right\rangle$ & $0.27 \pm 0.01$ & $0.29 \pm 0.01$ & $0.33 \pm 0.01$ & $0.14 \pm 0.01$ & $0.06 \pm 0.01$ \\
$\left\langle n_{c h}\left(n_{c h}-1\right)\right\rangle$ & $1.03 \pm 0.02$ & $4.22 \pm 0.07$ & $9.43 \pm 0.11$ & $1.08 \pm 0.03$ & $0.94 \pm 0.02$ \\
$\left\langle n_{\text {ch }} n_{\gamma}\right\rangle$ & $0.17 \pm 0.01$ & $0.43 \pm 0.01$ & $1.03 \pm 0.02$ & $0.09 \pm 0.01$ & $0.05 \pm 0.01$ \\
\hline$r(1,1)$ & $0.58 \pm 0.01$ & $0.69 \pm 0.01$ & $0.97 \pm 0.01$ & $0.62 \pm 0.02$ & $0.83 \pm 0.05$ \\
$r(2,1)$ & $0.44 \pm 0.03$ & $0.61 \pm 0.03$ & $1.03 \pm 0.02$ & $0.48 \pm 0.05$ & $0.92 \pm 0.11$ \\
$r(3,1)$ & $0.39 \pm 0.05$ & $0.57 \pm 0.04$ & $1.12 \pm 0.04$ & $0.39 \pm 0.08$ & $1.07 \pm 0.28$ \\
\hline$r(0,2)$ & $1.51 \pm 0.05$ & $1.27 \pm 0.06$ & $1.06 \pm 0.04$ & $1.32 \pm 0.12$ & $1.52 \pm 0.31$ \\
$r(1,2)$ & $0.67 \pm 0.05$ & $0.64 \pm 0.05$ & $1.10 \pm 0.06$ & $0.61 \pm 0.10$ & $0.79 \pm 0.30$ \\
$r(2,2)$ & $0.43 \pm 0.08$ & $0.41 \pm 0.06$ & $1.27 \pm 0.09$ & $0.34 \pm 0.13$ & $0.75 \pm 0.44$ \\
$r(0,3)$ & $2.77 \pm 0.34$ & $2.08 \pm 0.34$ & $1.17 \pm 0.21$ & $2.52 \pm 0.95$ & $3.06 \pm 3.04$ \\
$r(1,3)$ & $1.17 \pm 0.27$ & $0.79 \pm 0.16$ & $1.24 \pm 0.24$ & $0.75 \pm 0.38$ & $0.00 \pm 0.00$ \\
$r(0,4)$ & $6.04 \pm 1.84$ & $3.81 \pm 1.49$ & $1.66 \pm 0.98$ & $5.41 \pm 5.42$ & $0.00 \pm 0.00$ \\
\hline
\end{tabular}

Table 6.2: Results for varying parameters in the DCC generator. 


\begin{tabular}{|c|c|c|c|c|}
\hline fraction & $r_{1,1} \pm \sigma_{r_{1,1}}$ & $r_{2,1} \pm \sigma_{r_{2,1}}$ & $r_{3,1} \pm \sigma_{r_{3,1}}$ & \# events \\
\hline 0.00 & $1.01 \pm 0.02$ & $1.02 \pm 0.05$ & $1.09 \pm 0.14$ & 51471 \\
0.02 & $1.00 \pm 0.02$ & $1.00 \pm 0.05$ & $1.01 \pm 0.15$ & 51741 \\
0.05 & $0.97 \pm 0.02$ & $0.93 \pm 0.05$ & $0.95 \pm 0.10$ & 51741 \\
0.10 & $0.95 \pm 0.02$ & $0.89 \pm 0.04$ & $0.89 \pm 0.08$ & 51741 \\
0.20 & $0.93 \pm 0.02$ & $0.83 \pm 0.04$ & $0.77 \pm 0.07$ & 51741 \\
0.50 & $0.84 \pm 0.01$ & $0.71 \pm 0.03$ & $0.68 \pm 0.06$ & 40000 \\
1.00 & $0.74 \pm 0.01$ & $0.60 \pm 0.03$ & $0.55 \pm 0.06$ & 20000 \\
\hline
\end{tabular}

Table 6.3: The effect on the $r_{i, 1}$ of an admixture of DCC and generic (PYTHIA) events. DCC domains from the DCC generator/GEANT are added to various fractions of random PYTHIA/GEANT events. The first column represents the fraction of events in which a DCC is overlaying a generic event. A DCC fraction of 1 means that DCC has been added to every event, not that the events are pure DCC as in Table 6.1. 


\begin{tabular}{|c|c|c|c|c|c|c|c|c|c|c|}
\hline & \multicolumn{9}{|c|}{$n_{\gamma}$} \\
\hline & & 0 & 1 & 2 & 3 & 4 & 5 & 6 & 7 & 8 \\
\hline \multirow{9}{*}{$n_{c h}$} & 0 & 834625 & 126160 & 16739 & 2515 & $\overline{454}$ & 67 & 10 & 8 & 1 \\
\hline & 1 & 355999 & 62717 & 10398 & 1732 & 272 & 56 & 12 & 1 & 0 \\
\hline & 2 & 87117 & 19140 & 3580 & 639 & 148 & 31 & 4 & 0 & 0 \\
\hline & 3 & 17786 & 4771 & 1016 & 208 & 34 & 9 & 0 & 0 & 0 \\
\hline & 4 & 3350 & 943 & 224 & 48 & 10 & 2 & 0 & 0 & 0 \\
\hline & 5 & 516 & 182 & 49 & 7 & 4 & 0 & 0 & 0 & 0 \\
\hline & 6 & 88 & 38 & 7 & 3 & 0 & 0 & 0 & 0 & 0 \\
\hline & 7 & 10 & 6 & 1 & 0 & 0 & 0 & 0 & 0 & 0 \\
\hline & 8 & 0 & 1 & 0 & 0 & 0 & 0 & 0 & 0 & 0 \\
\hline
\end{tabular}

Table 6.4: Number of events with a given $n_{c h}, n_{\gamma}$. 


\begin{tabular}{|c|c|c|c|c|}
\hline & all events & ktag & $\overline{\mathrm{n}}$ hcal & $\overline{\mathrm{p}}$ hcal \\
\hline \# events & 1551738 & 21412 & 27807 & 42449 \\
\hline$\left\langle n_{c h}\right\rangle$ & $0.4814 \pm 0.0006$ & $0.407 \pm 0.005$ & $0.422 \pm 0.004$ & $0.437 \pm 0.003$ \\
$\left\langle n_{\gamma}\right\rangle$ & $0.1922 \pm 0.0004$ & $0.167 \pm 0.003$ & $0.173 \pm 0.003$ & $0.183 \pm 0.002$ \\
$\left\langle n_{\text {ch }}\left(n_{\text {ch }}-1\right)\right\rangle$ & $0.2830 \pm 0.0010$ & $0.195 \pm 0.007$ & $0.213 \pm 0.006$ & $0.227 \pm 0.005$ \\
$\left\langle n_{\text {ch }} n_{\gamma}\right\rangle$ & $0.1156 \pm 0.0004$ & $0.084 \pm 0.003$ & $0.092 \pm 0.003$ & $0.103 \pm 0.002$ \\
\hline$r(1,1)$ & $1.0228 \pm 0.0035$ & $1.052 \pm 0.036$ & $1.051 \pm 0.031$ & $1.076 \pm 0.024$ \\
$r(2,1)$ & $1.0320 \pm 0.0097$ & $1.134 \pm 0.107$ & $1.042 \pm 0.083$ & $1.116 \pm 0.069$ \\
$r(3,1)$ & $1.0563 \pm 0.0251$ & $1.302 \pm 0.281$ & $1.220 \pm 0.211$ & $1.180 \pm 0.173$ \\
$r(4,1)$ & $1.1183 \pm 0.0612$ & $1.802 \pm 0.637$ & $2.102 \pm 0.599$ & $1.370 \pm 0.413$ \\
$r(5,1)$ & $1.3382 \pm 0.1436$ & & & $1.721 \pm 0.911$ \\
$r(6,1)$ & $2.0758 \pm 0.4159$ & & & \\
$r(7,1)$ & $5.0105 \pm 5.6885$ & & & \\
\hline$r(0,2)$ & $1.5793 \pm 0.0092$ & $1.647 \pm 0.090$ & $1.683 \pm 0.077$ & $1.658 \pm 0.059$ \\
$r(1,2)$ & $1.5632 \pm 0.0204$ & $1.623 \pm 0.217$ & $1.700 \pm 0.176$ & $1.675 \pm 0.152$ \\
$r(2,2)$ & $1.5538 \pm 0.0497$ & $1.914 \pm 0.616$ & $1.908 \pm 0.474$ & $1.933 \pm 0.441$ \\
$r(0,3)$ & $3.3711 \pm 0.0759$ & $3.378 \pm 0.627$ & $3.359 \pm 0.545$ & $3.333 \pm 0.390$ \\
$r(1,3)$ & $3.1115 \pm 0.1210$ & $3.335 \pm 1.165$ & $3.196 \pm 0.895$ & $3.367 \pm 0.958$ \\
$r(0,4)$ & $8.9729 \pm 0.6201$ & $6.675 \pm 2.934$ & $7.266 \pm 2.980$ & $6.236 \pm 1.780$ \\
\hline
\end{tabular}

Table 6.5: Values of $r_{i, j}$ for all lead-in events and for those with diffractive tags. 


\begin{tabular}{|c|c|c|c|c|c|}
\hline bin & $\left\langle n_{c h}\right\rangle$ & $\left\langle n_{\gamma}\right\rangle$ & $r_{1,1}$ & $r_{2,1}$ & $r_{3,1}$ \\
\hline 1 & $0.364 \pm 0.002$ & $0.145 \pm 0.002$ & $1.02 \pm 0.02$ & $0.97 \pm 0.06$ & $0.87 \pm 0.14$ \\
2 & $0.401 \pm 0.002$ & $0.165 \pm 0.002$ & $1.00 \pm 0.02$ & $1.00 \pm 0.05$ & $1.04 \pm 0.14$ \\
3 & $0.426 \pm 0.002$ & $0.173 \pm 0.002$ & $1.03 \pm 0.02$ & $1.11 \pm 0.05$ & $1.28 \pm 0.13$ \\
4 & $0.449 \pm 0.002$ & $0.183 \pm 0.002$ & $1.00 \pm 0.02$ & $1.05 \pm 0.05$ & $1.10 \pm 0.13$ \\
5 & $0.468 \pm 0.003$ & $0.190 \pm 0.002$ & $1.03 \pm 0.02$ & $1.10 \pm 0.05$ & $1.17 \pm 0.14$ \\
6 & $0.493 \pm 0.003$ & $0.195 \pm 0.002$ & $1.02 \pm 0.02$ & $1.01 \pm 0.04$ & $1.05 \pm 0.11$ \\
7 & $0.503 \pm 0.003$ & $0.202 \pm 0.002$ & $1.04 \pm 0.02$ & $1.03 \pm 0.05$ & $0.98 \pm 0.12$ \\
8 & $0.531 \pm 0.003$ & $0.215 \pm 0.002$ & $1.01 \pm 0.01$ & $1.00 \pm 0.04$ & $0.97 \pm 0.09$ \\
9 & $0.555 \pm 0.003$ & $0.221 \pm 0.002$ & $1.03 \pm 0.01$ & $1.06 \pm 0.04$ & $1.09 \pm 0.11$ \\
10 & $0.603 \pm 0.003$ & $0.237 \pm 0.002$ & $1.03 \pm 0.01$ & $1.02 \pm 0.03$ & $1.01 \pm 0.07$ \\
\hline
\end{tabular}

Table 6.6: Mean multiplicities and robust observables for bins of pbar multiplicities each containing $10 \%$ of the events. The pbar multiplicity increases with increasing bin number 1-10. 


\section{Chapter 7}

\section{Conclusions}

MiniMax ran successfully with many different detector configurations for a period of about two years. During this time, much was learned which should prove useful for the operation and design of future detectors in the forward region. More than $1.5 \times 10^{6}$ events with lead in and almost $10^{6}$ events with lead out from clean runs when the detector was running properly have been analyzed for the work presented here.

The GEANT simulation does not accurately represent the amount of background seen in the wire chambers from interactions in material surrounding the detector. Even with all of the material included in the simulation, the mean number of hits in the MWPC's is low by about a factor of two. However, the PYTHIA/GEANT output is useful for setting upper limits on how well the data analysis tools are able to reconstruct events. Based on the efficiencies and numbers of fakes discussed in Sec. 4.3, the tracker and 
vertexer appear to work quite well.

The simulations also show that the robust observables of Chapter 5 are insensitive to detector effects and physics complications, as advertised. The PYTHIA/GEANT simulation, which includes a photon conversion efficiency of slightly better than $50 \%$, an efficiency of $80 \%$ for detecting converted photons, resonance production, simulations of detector effects, and many other features, matches the predictions of a simple binomial model. At least for DCC modeled as in Sec. 3.3, the robust observables should be useful in distinguishing DCC from generic production.

We have found no evidence for DCC in the total sample of events or in diffractive events. Limits on the amount of DCC which could be present without being detected depend on the models of DCC (e.g. the $p_{T}$ of the DCC pions) and of combining DCC with generic production.

Raw measurements of $d N_{c h} / d \eta$ and $d N_{\gamma} / d \eta$ have been made in a previously unexplored region of phase space, from which actual distributions can be derived, taking into account detection efficiencies and fakes, and trigger efficiencies. Further work on modeling fakes in the data is necessary before this can be done. 


\section{Appendix A}

\section{Uncertainty Calculations}

The normalized factorial moments are defined as

$$
F_{j, l}=\frac{\left\langle n_{c}\left(n_{c}-1\right) \ldots\left(n_{c}-j+1\right) n_{g}\left(n_{g}-1\right) \ldots\left(n_{g}-l+1\right)\right\rangle}{\left\langle n_{c}\right\rangle^{j}\left\langle n_{g}\right\rangle^{l}}
$$

where $\langle\mathcal{O}\rangle=\frac{1}{N} \sum_{n_{c}, n_{g}} \mathcal{O N}\left(n_{c}, n_{g}\right), N=\sum_{n_{c}, n_{g}} \mathcal{N}\left(n_{c}, n_{g}\right)$, and $\mathcal{N}\left(n_{c}, n_{g}\right)$ is the number of events with $n_{c}$ charged tracks and $n_{g}$ gammas.

We assume that $\sigma_{\mathcal{N}\left(n_{c}, n_{g}\right)}^{2}=\mathcal{N}\left(n_{c}, n_{g}\right)$. The uncertainty in $F_{j, l}$ is then given by

$$
\begin{gathered}
\sigma_{F_{j, l}}^{2}=\frac{1}{N}\left[\frac{\left\langle\left[n_{c} \ldots\left(n_{c}-j+1\right) n_{g} \ldots\left(n_{g}-l+1\right)\right]^{2}\right\rangle}{\left\langle n_{c}\right\rangle^{2 j}\left\langle n_{g}\right\rangle^{2 l}}-(j+l-1)^{2} F_{j, l}{ }^{2}\right. \\
+j^{2} \frac{\left\langle n_{c}^{2}\right\rangle}{\left\langle n_{c}\right\rangle^{2}} F_{j, l}{ }^{2}+l^{2} \frac{\left\langle n_{g}^{2}\right\rangle}{\left\langle n_{g}\right\rangle^{2}} F_{j, l}{ }^{2}+2 j l \frac{\left\langle n_{c} n_{g}\right\rangle}{\left\langle n_{c}\right\rangle\left\langle n_{g}\right\rangle} F_{j, l}{ }^{2}
\end{gathered}
$$




$$
\begin{aligned}
& -2 j \frac{\left\langle n_{c}\left[n_{c} \ldots\left(n_{c}-j+1\right) n_{g} \ldots\left(n_{g}-l+1\right)\right]\right\rangle}{\left\langle n_{c}\right\rangle^{j+1}\left\langle n_{g}\right\rangle^{l}} F_{j, l} \\
& \left.-2 l \frac{\left\langle n_{g}\left[n_{c} \ldots\left(n_{c}-j+1\right) n_{g} \ldots\left(n_{g}-l+1\right)\right]\right\rangle}{\left\langle n_{c}\right\rangle^{j}\left\langle n_{g}\right\rangle^{l+1}} F_{j, l}\right]
\end{aligned}
$$

In order to find the correlated uncertainty for any two $F$ 's we define

$$
x=F_{j, l}+F_{i, m}
$$

which has uncertainty given by

$$
\sigma_{x}^{2}=\sigma_{F_{j, l}}^{2}+\sigma_{F_{i, m}}^{2}+2 \sigma_{F_{j, l} F_{i, m}}
$$

Writing the $F$ 's in terms of the $\mathcal{N}\left(n_{c}, n_{g}\right)$, we get

$$
\begin{gathered}
\sigma_{x}^{2}=\sum_{n_{c}^{\prime}, n_{g}^{\prime}}\left[\frac{n_{c}^{\prime} \ldots\left(n_{c}^{\prime}-j+1\right) n_{g}^{\prime} \ldots\left(n_{g}^{\prime}-l+1\right)}{N\left\langle n_{c}\right\rangle^{j}\left\langle n_{g}\right\rangle^{l}}+(j+l-1) \frac{F_{j, l}}{N}\right. \\
-j \frac{n_{c}^{\prime} F_{j, l}}{N\left\langle n_{c}\right\rangle}-l \frac{n_{g}^{\prime} F_{j, l}}{N\left\langle n_{g}\right\rangle} \\
+\frac{n_{c}^{\prime} \ldots\left(n_{c}^{\prime}-i+1\right) n_{g}^{\prime} \ldots\left(n_{g}^{\prime}-m+1\right)}{N\left\langle n_{c}\right\rangle^{i}\left\langle n_{g}\right\rangle^{m}}+(i+m-1) \frac{F_{i, m}}{N}
\end{gathered}
$$


182

$$
\left.-i \frac{n_{c}^{\prime} F_{i, m}}{N\left\langle n_{c}\right\rangle}-m \frac{n_{g}^{\prime} F_{i, m}}{N\left\langle n_{g}\right\rangle}\right]^{2} \mathcal{N}\left(n_{c}^{\prime}, n_{g}^{\prime}\right)
$$

and we can pick out the correlation terms to find

$$
\begin{aligned}
& \sigma_{F_{j, l} F_{i, m}=\frac{1}{N}[}\left[\frac{\left\langle\left[n_{c} \ldots\left(n_{c}-j+1\right) n_{g} \ldots\left(n_{g}-l+1\right)\right]\left[n_{c} \ldots\left(n_{c}-i+1\right) n_{g} \ldots\left(n_{g}-m+1\right)\right]\right\rangle}{\left\langle n_{c}\right\rangle^{j+i}\left\langle n_{g}\right\rangle^{l+m}}\right. \\
&-i \frac{\left\langle n_{c}\left[n_{c} \ldots\left(n_{c}-j+1\right) n_{g} \ldots\left(n_{g}-l+1\right)\right]\right\rangle}{\left\langle n_{c}\right\rangle^{j+1}\left\langle n_{g}\right\rangle^{l}} F_{i, m} \\
&-m \frac{\left\langle n_{g}\left[n_{c} \ldots\left(n_{c}-j+1\right) n_{g} \ldots\left(n_{g}-l+1\right)\right]\right\rangle}{\left\langle n_{c}\right\rangle^{j}\left\langle n_{g}\right\rangle^{l+1}} F_{i, m} \\
&-j \frac{\left\langle n_{c}\left[n_{c} \ldots\left(n_{c}-i+1\right) n_{g} \ldots\left(n_{g}-m+1\right)\right]\right\rangle}{\left\langle n_{c}\right\rangle^{i+1}\left\langle n_{g}\right\rangle^{m}} F_{j, l} \\
&-l \frac{\left\langle n_{g}\left[n_{c} \ldots\left(n_{c}-i+1\right) n_{g} \ldots\left(n_{g}-m+1\right)\right]\right\rangle}{\left\langle n_{c}\right\rangle^{i}\left\langle n_{g}\right\rangle^{m+1}} F_{j, l} \\
&+i j \frac{\left\langle n_{c}^{2}\right\rangle}{\left\langle n_{c}\right\rangle^{2}} F_{j, l} F_{i, m}+l m \frac{\left\langle n_{g}^{2}\right\rangle}{\left\langle n_{g}\right\rangle^{2}} F_{j, l} F_{i, m}
\end{aligned}
$$




$$
\left.+(j m+i l) \frac{\left\langle n_{c} n_{g}\right\rangle}{\left\langle n_{c}\right\rangle\left\langle n_{g}\right\rangle} F_{j, l} F_{i, m}-(j+l-1)(i+m-1) F_{j, l} F_{i, m}\right]
$$

The generalized $r$ defined by

$$
r_{j, l}=\frac{F_{j, l}}{F_{j+l, 0}}
$$

has uncertainty given by

$$
\sigma_{r_{j, l}}^{2}=\left(\frac{1}{F_{j+l, 0}}\right)^{2} \sigma_{F_{j, l}}^{2}+\left(-\frac{r_{j l}}{F_{j+l, 0}}\right)^{2} \sigma_{F_{j+l, 0}}^{2}+2\left(-\frac{r_{j l}}{F_{j+l, 0}^{2}}\right) \sigma_{F_{j, l} F_{j+l, 0}}
$$




\section{Appendix B}

\section{Track fitting code}

\section{B.1 Track fitter: fit.f}

SUBROUTINE fit_init

IMPLICIT NONE

INTEGER I, NW

REAL sigsq, wiresp, pi, uang, sum, cu, su

REAL UO (24), $\mathrm{VO}(24)$, ang (24), Z(24)

common/cham_param/MN, sigsq, wiresp, pi , uang, sum, cu, su , vo , vo, ang , $Z$

C position of the center of wire 0 and angle of rotation in $(x, y, z)$

real $\mathrm{zz}(24) / 123.69,126.57,129.69,132.57$,

$+$

$+$

$+$

$+$

$+$

real $\mathrm{xx0}(24) / 2.806,10.117,0.853,11.792$,

$+$

$+$

$+$

$+$

$+$

$$
\text { real yyo(24)/11.653,11.032,10.870,9.255, }
$$

$10.910,9.193,11.700,1.813$,

$+$

$+$

$135.69,138.69,141.69,144.69$,

$167.09,169.89,172.84,176.09$,

$178.84,181.84,185.09,189.65$,

$194.29,197.29,200.29,203.29$,

$206.16,209.16,212.41,215.41 /$

$9.997,0.818,10.076,1.252$,

$1.692,10.090,10.970,1.535$,

$11.199,12.360,11.424,3.366$,

$11.602,11.327,2.814,12.648$,

$12.056,3.560,3.140,2.681 /$

$2.568,12.305,11.702,3.508$, 


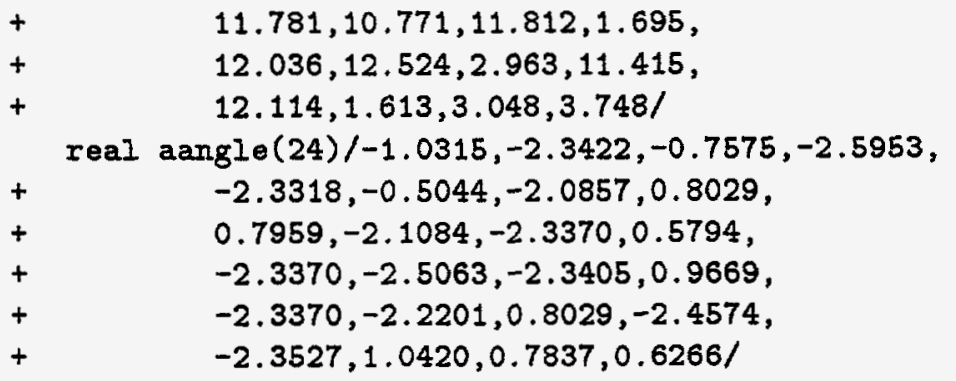

sigsq=1./12. !uncer in location of track (wire spacings) for each hit wiresp=0.1 !wire spacing in inches

$\mathrm{NN}=4$

$\mathrm{pi}=3.141592654$

uang $=45.43 * \mathrm{pi} / 180$. !taken as mean angle of "u chambers" cu=cos (uang)

$\operatorname{su}=\sin$ (uang)

do $i=1,24$

UO $(i)=\operatorname{xxO}(i) * c u+y y O(i) * s u \quad$ !u,v of center of wire 0

$\mathrm{VO}(i)=-\mathrm{xxO}(i) * \mathrm{su}+\mathrm{yyO}(i) * \mathrm{cu}$

$z(i)=\mathbf{z z}(i)$

if (aangle (i).lt. $2 * \mathrm{p} i$ ) aangle(i) =aangle(i) $+2 * p i$

$\operatorname{ang}(i)=\operatorname{aangle}(i)-$ uang

$\operatorname{ang}(i)=\operatorname{ang}(i)-p i / 2$.

enddo

RETURN

END

SUBROUTINE fit_wires

IMPLICIT NONE

INTEGER I, J,K,I, num

INTEGER icham, ichlast, ncham, iwire, nwires (24), iicham(24)

REAL wire

REAL anires $(24,128)$

$\operatorname{REAL} * 8 \quad X(4,4), X X(100,4), Y(4), Y Y(100), a(4), X I N V(4,4)$

REAL* 8 INDEX (4), D 


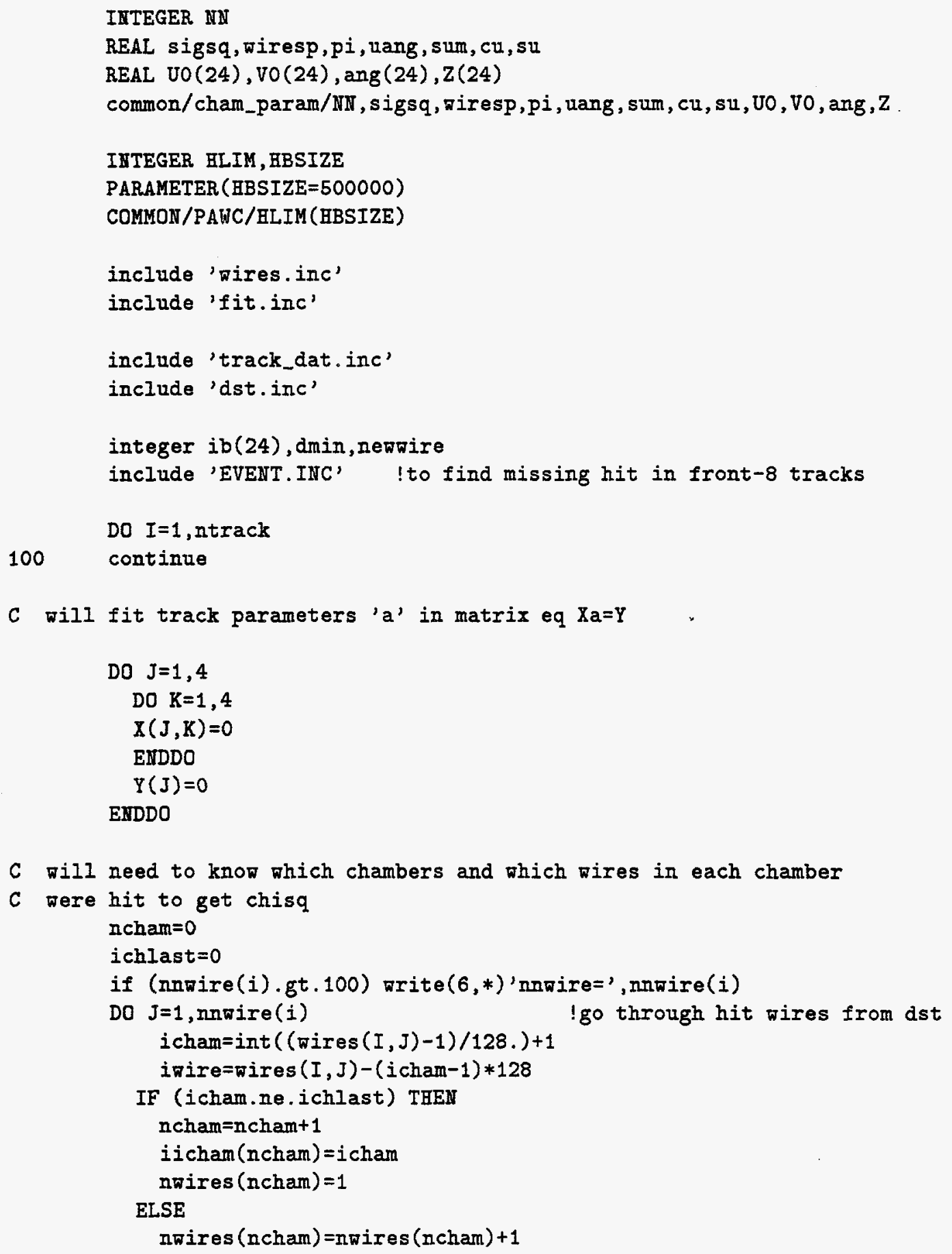




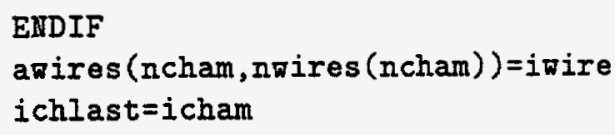

$C$ invert $X$

$$
\text { DO } J=1,4
$$

C fill fit.inc 


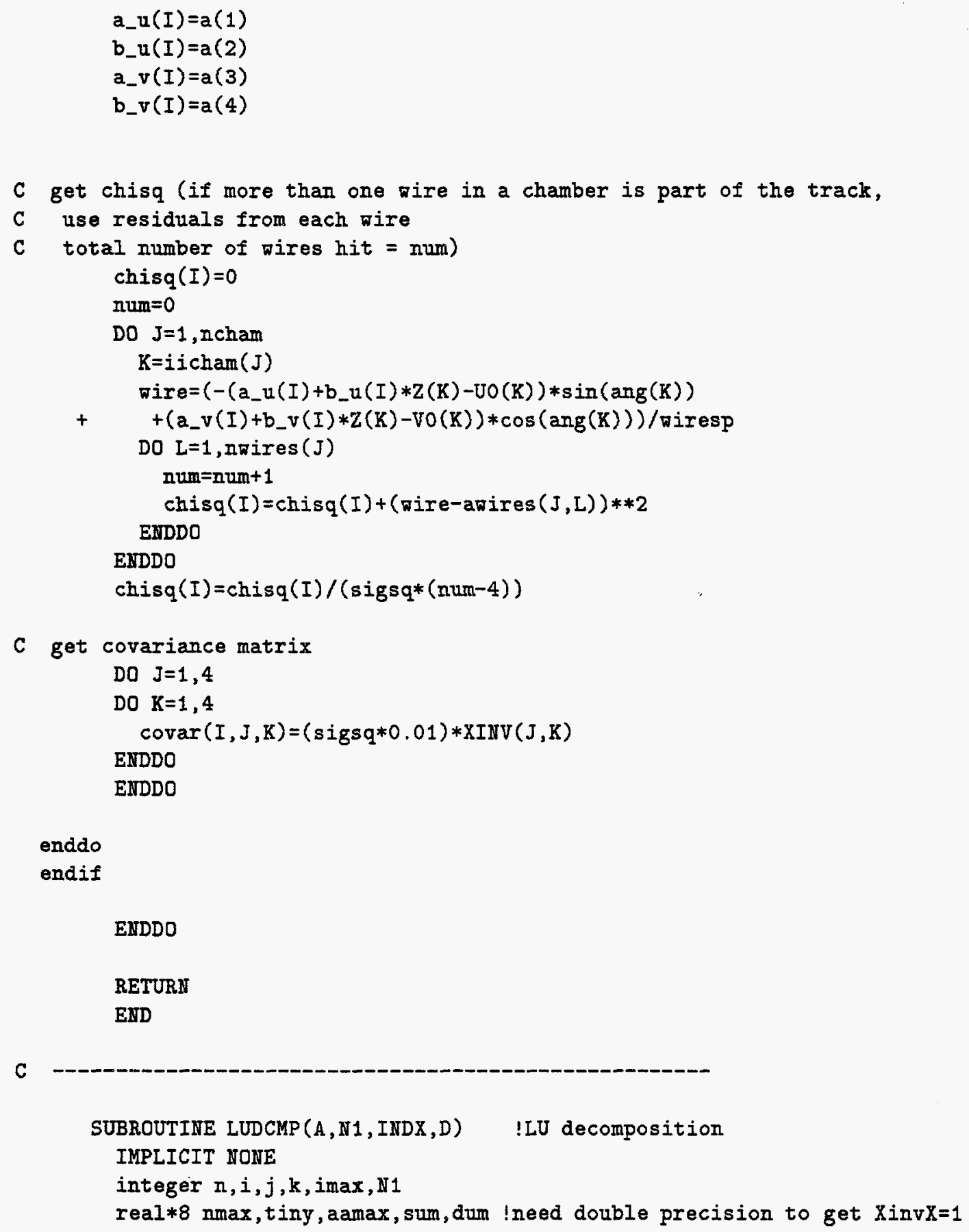


PARAMETER (NMAX $=46, T I N Y=1, O E-20)$

$R E A L * 8$ NP , D , A (4, 4), INDX (4), VV(4)

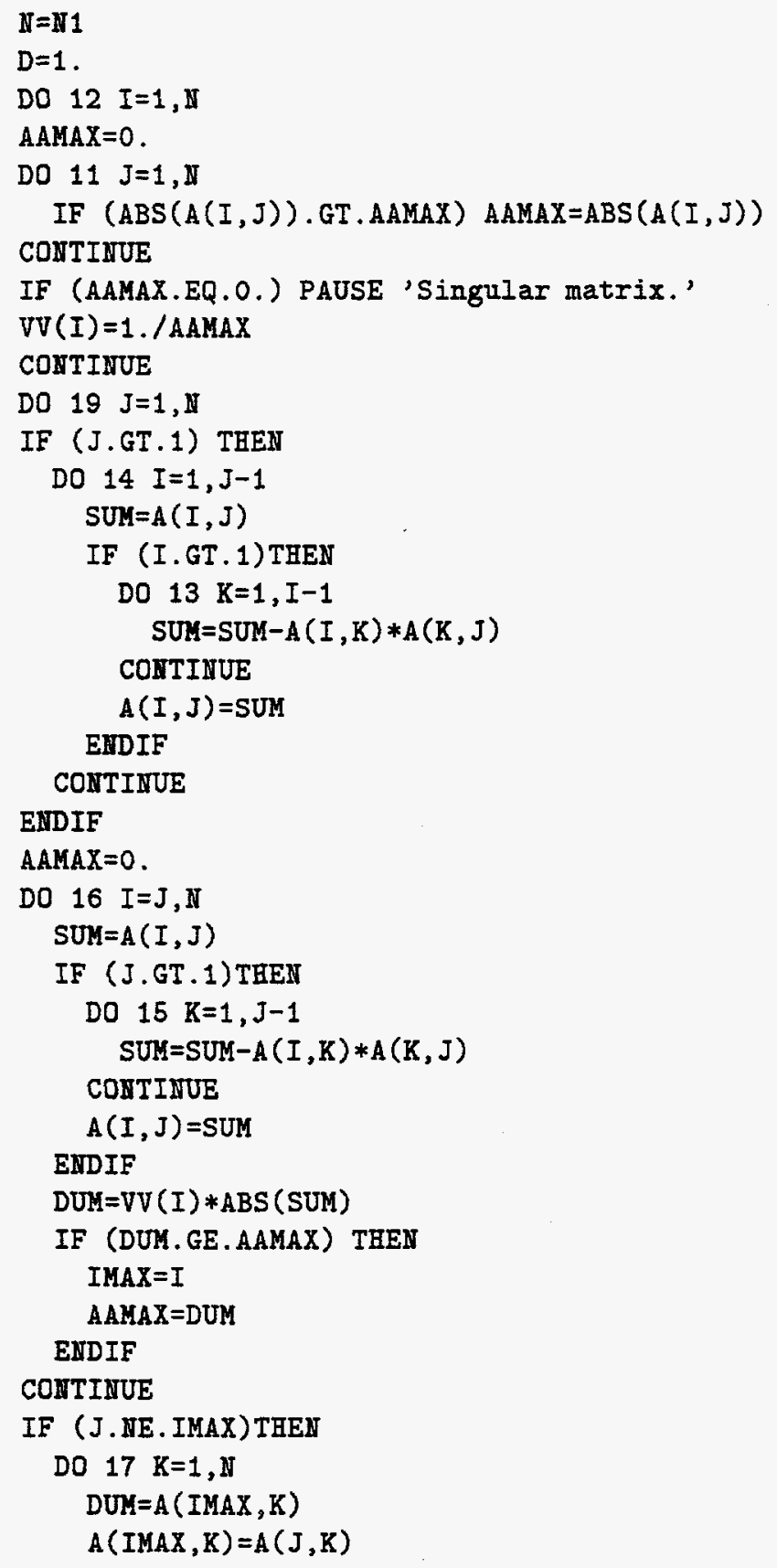



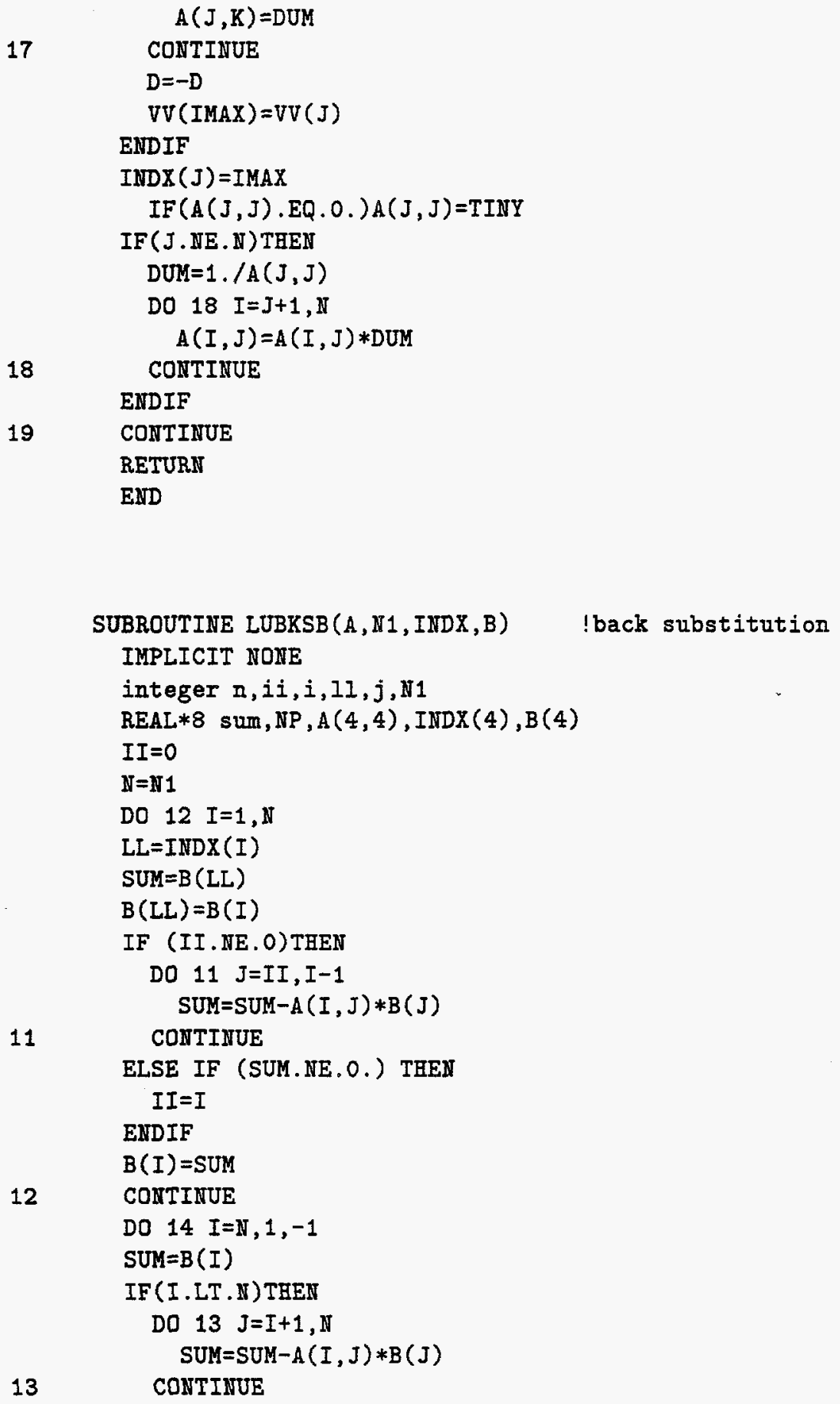


$\begin{array}{ll} & \text { ENDIF } \\ & \mathrm{B}(I)=S U M / A(I, I) \\ \text { CONTINUE } \\ \text { RETURN } \\ \text { END }\end{array}$

C-

C wires.inc (filled by eg vertexer.f)

integer ntrack, nnwire (200), wires $(200,100)$

common/riresforfit/ntrack,nnwire, wires

$C$ (must be filled by user unless using the vertexer)

C ntrack $=\#$ tracks

C nnwire(i)=\# vires in track $i$

C wires $(i, j)=j$ th wire of $i$ th track in usual format (icham-1)*128+iwire

c-con

C fit.inc (filled by fit.f)

REAL a_u(200), b_u(200), a_v(200), b_v(200), chisq(200)

REAL COVAR $(200,4,4)$

integer current_evnum

common/track_param/a_u, b_u, a_v, b_v, chisq, covar, current_evnum

C(determined by fit.f)

C $u=a_{-} u+b_{-} u * z, v=a_{-} v+b_{-} v * z$

C

C for covariance matrix, indices go like

c $1=a_{-} u, 2=b_{-} u, 3=a_{-} v, 4=b_{-} v$ 


\title{
B.2 Code for correcting tracks with double hits in the non- $u$ chambers: refit.f
}

\author{
SUBROUTINE refit
}

IMPLICIT NONE

INTEGER I, J,K, L,M

INTEGER icham, ichlast, ncham, iwire,nøires (24), iicham(24)

INTEGER NNT

REAL sigsq, wiresp, pi, uang, sum, cu, su

REAL UO(24), VO(24), ang (24), Z(24)

common/cham_param/WN, sigsq, wiresp, pi, uang, sum, cu, su, vo, vo, ang , $Z$

integer itrack

real av, bv, $v(24), u(24)$, vlead, ucham (24)

real sz, szz, sv, szv, cth(24), sth(24), denom, predwire, wire $(18000,24)$

real sigth, thgam, thtail

integer nwirepl(24), inirepl $(24,10)$, nFire, icomb

integer $i$ ( $(8), i 1, i 2, i 3, i 4, i 5, i 6, i 7, i 8$

integer ncomb, ichi, ipoint

real chi(18000), point (18000), chimin, pointmin

integer ntr,hit2

integer nch1, nch2

common/newnch/nch1,nch2

include 'vires.inc'

include 'fit.inc'

include 'track_dat.inc'

include 'dst.inc'

C get u chambers

do $j=1,24$

$\operatorname{ucham}(j)=0$

enddo

$\operatorname{ucham}(2)=1$

$\operatorname{ucham}(5)=1$

$\operatorname{ucham}(8)=1$

$\operatorname{ucham}(9)=1$ 


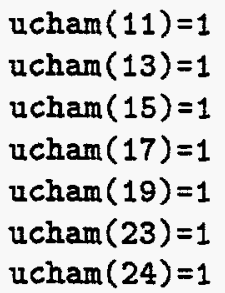

C

will need orientation of non- $u$ chambers to fit in $v$ do $k=1,24$

if (ucham(k).eq.0) then $\operatorname{sth}(k)=\sin (\operatorname{ang}(k))$

C check number of wires hit per plane

C if 2 wires hit are separated by more than 3 wires in any plane, refit do $i=1$,ntrack

C how many chambers have "double hits" and which wires are involved ncham $=0$

do $\mathrm{k}=1,24$

$n$ irepl $(k)=0$

enddo

ichlast $=0$

DO $J=1$, nnwire(i)

icham $=\operatorname{int}((\operatorname{aires}(I, J)-1) / 128)+$. 


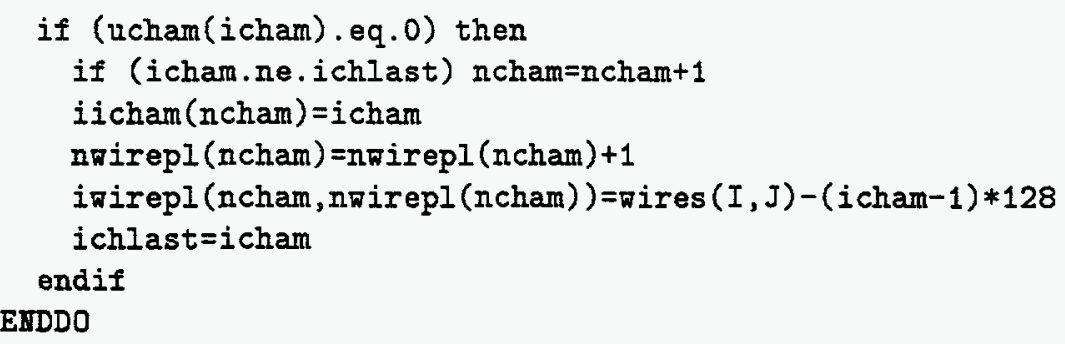

C cycle thru all wires!!!

$C$ and find all possible combinations with 1 wire per plane ncomb $=0$

if (ncham.eq.3) then

do $i 1=1, n$ irepl(1)

$i n(1)=i 1$

do $i 2=1$, nwirepl (2)

$i v(2)=i 2$

do $i 3=1, n$ nirepl $(3)$

$i m(3)=i 3$

ncomb $=$ ncomb +1

do $j=1$, ncham

if (ncomb.le.18000) $\operatorname{mire}(n \operatorname{comb}, j)=i \operatorname{irepl}(j, i \pi(j))$ enddo

enddo

endio

enddo

endif

if (ncham.eq.4) then

do $i 1=1$, nwirepl(1)

$i v(1)=i 1$

do i2=1, nwirepl(2)

in $(2)=i 2$

do $i 3=1$, nwirepl(3)

in $(3)=i 3$

do $i 4=1$, nwirepl (4)

in $(4)=i 4$

ncomb $=$ ncomb +1

do $j=1$, ncham

if (ncomb.le.18000) $\operatorname{wire}(n \operatorname{comb}, j)=i$ wirepl $(j, i \pi(j))$

enddo

encido

enddo

enddo

enddo 


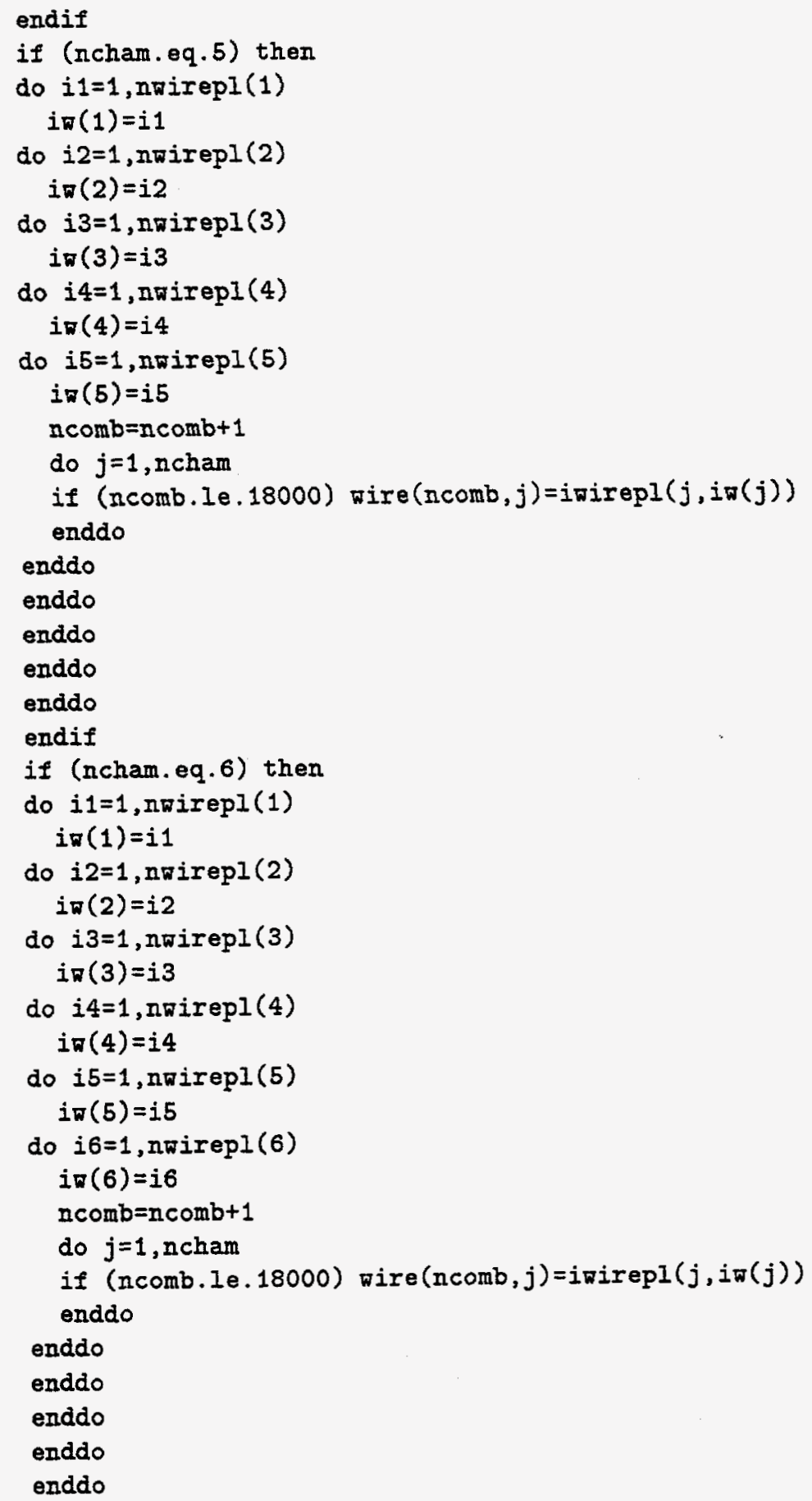




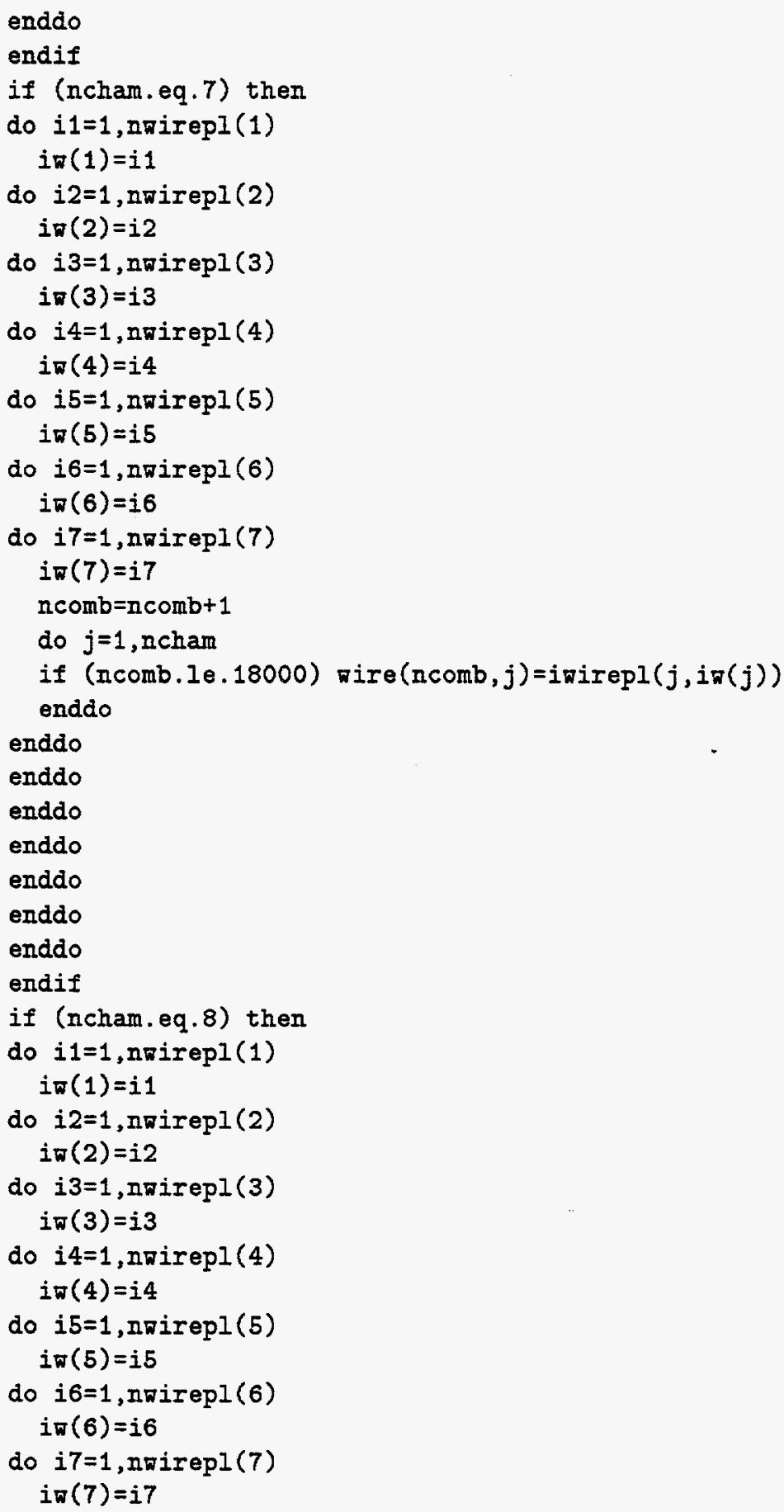




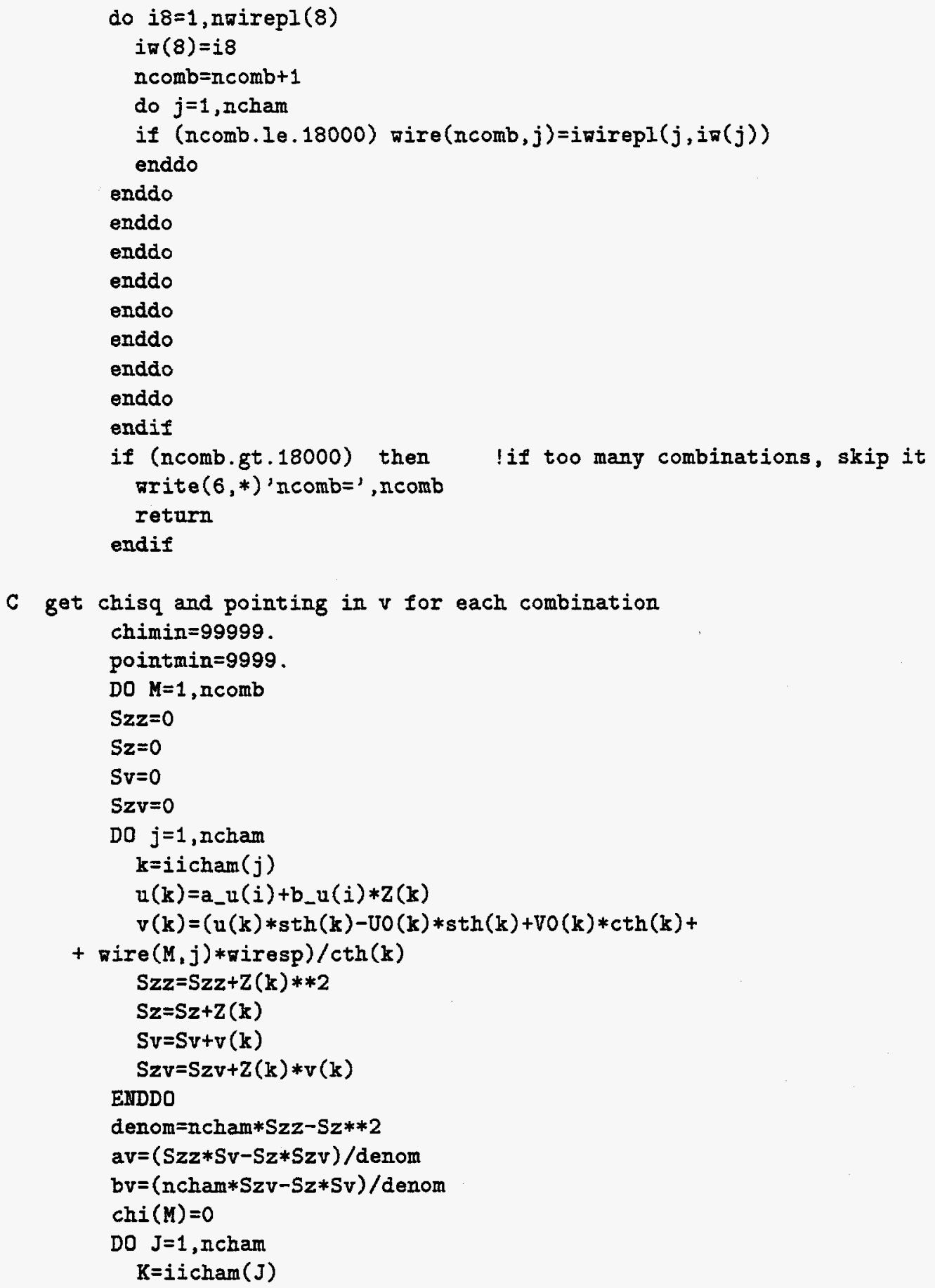




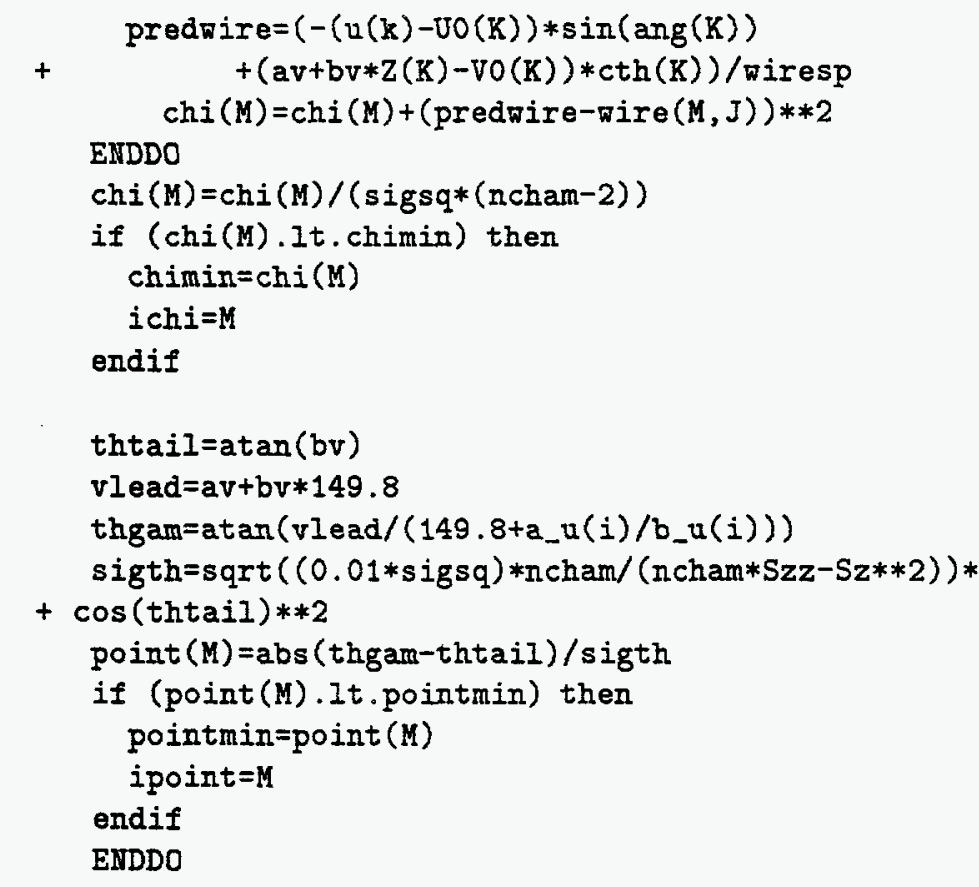

C require vpoint $<8$ and chisq $<7$, give preference to best pointing if (ncomb.gt.0) then

$i c o m b=0$

if ((pointmin.1t.8.).and.(chi(ipoint).1t.7)) then icomb=ipoint 


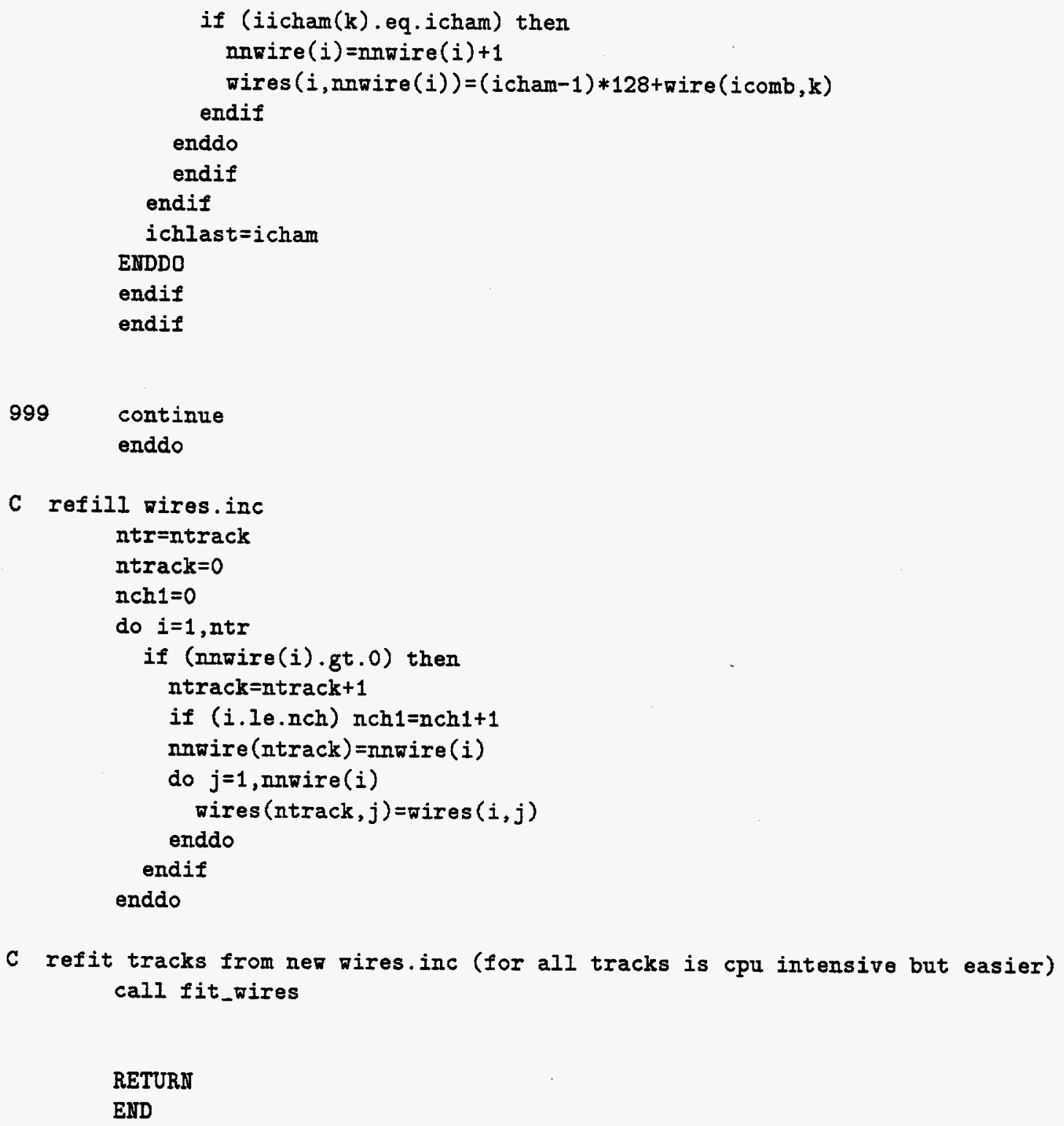




\section{Appendix C}

\section{Vertexer code}

\section{C.1 Vertexer: vertexer.f}

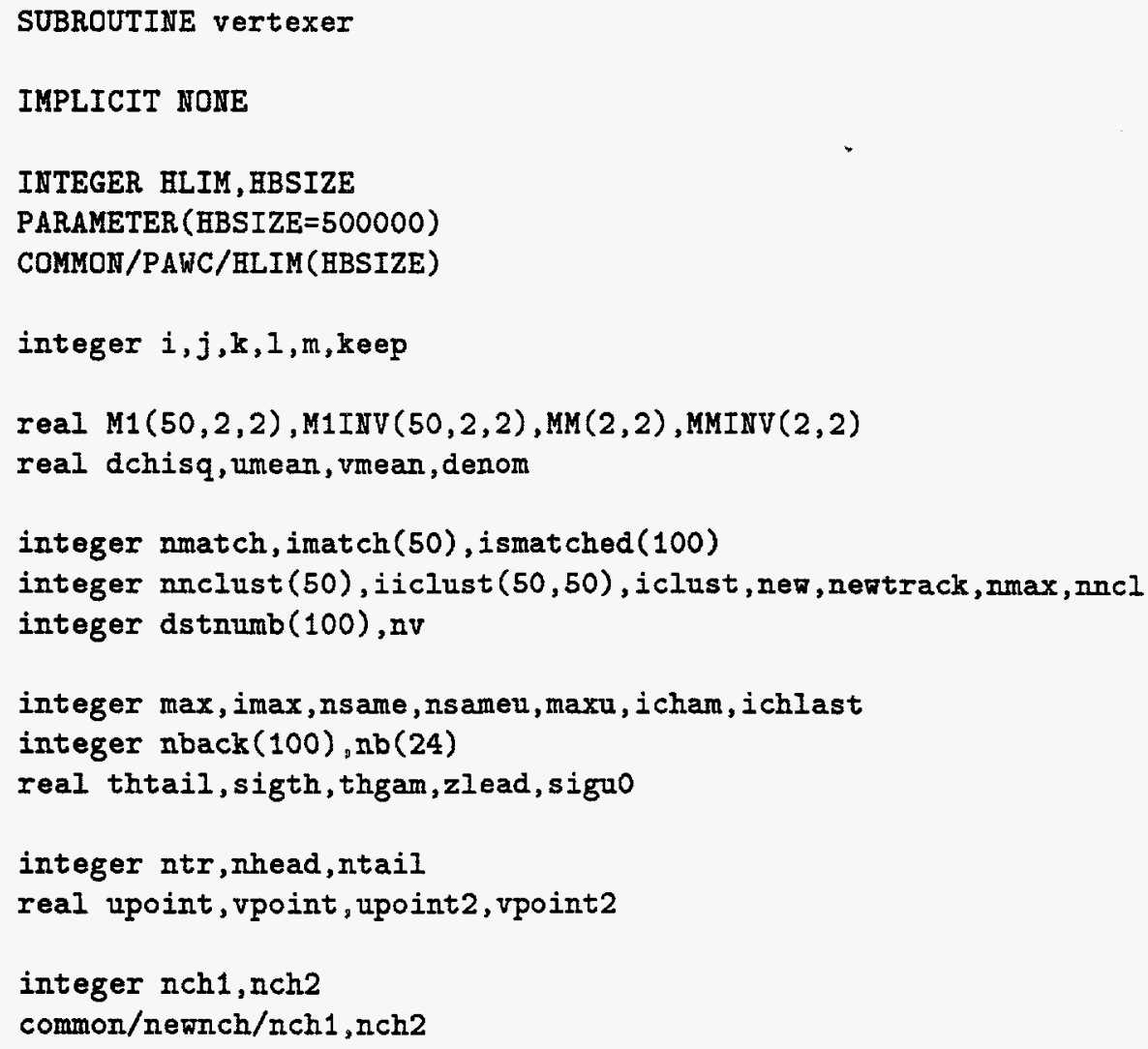


real uold1, vold 1

real dumin, dvmin, dmin, $d u, d v$, vold, vold

common/taildist/dumin, dvmin, dmin, du, dv, uold, vold

include 'fit.inc'

include ' $\nabla i r e s . i n c$ '

include 'vrtx.inc'

include 'VRTCS.INC'

include 'track_dat.inc'

include 'dst.inc'

zlead $=149.82$

C fill wires.inc with all track segments

ntrack $=0$

DO $k=1, n c h+n n u$

C get number of wires (don't distinguish betwen heads or tails)

IF (k.le.nch) THEN

ntrack=ntrack+1

if (ntrack.gt.100) write(6,*)ntrack

dstnumb (ntrack) $=\mathbf{k}$

nnwire $(k)=\operatorname{int}(\operatorname{charged}(k, 6))$

DO $J=1$, nnwire (ntrack)

$\operatorname{\text {ires}}(k, j)=$ wires_c $(k, J)$

EIDDO

ELSE

DO $\mathrm{L}=9,24$

$n b(L)=0$

ENDDO

DO $j=1$, neutral $(k-n c h, 6)$

icham $=$ int $(($ wires_n $(k-n c h, j)-1) / 128)+$.

$\mathrm{nb}$ (icham) $=1$

EIIDDO

$\operatorname{nback}($ ntrack +1$)=0$

DO $\mathrm{L}=9,24$

$\operatorname{nback}($ ntrack +1$)=\operatorname{nback}($ ntrack +1$)+n b(L)$

ENDDO

if (nback(ntrack+1).ge.8) then ! can change to exclude mid-8 tracks ntrack $=$ ntrack +1

dstnumb (ntrack) $=\mathrm{k}$

nnwire (ntrack) =int (neutral $(\mathrm{k}-\mathrm{nch}, 6)$ )

DO $\mathrm{J}=1, \mathrm{nn} \times \operatorname{ire}$ (ntrack) 
ENDDO

endif

EIDIF

ENDDO

C fit tracks

call fit_wires

call refit

C get position of tracks at lead and uncertainty in pos call vrtx(zlead)

C remove heads which don't point and tracks which don't hit lead ntr=ntrack

ntrack $=0$

nch $2=0$

do $i=1$,ntr

if (i.le.nch1) then

upoint $=-a_{-} u(i) / b_{-} u(i)$

else

upoint $=0$

endif

$C$ for now, don't demand tails point, loose on heads

if ((upoint.gt. -50). and. (upoint.1t.60.)) then if (i.le.nch1) then

thtail=atan (b_v(i))

thgamatan $\left(v(i) /\left(z l e a d+a \_u(i) / b_{-} u(i)\right)\right)$

sigth=sqrt $(\operatorname{covar}(i, 4,4)) * \cos ($ thtail $) * * 2$

vpoint=abs (thgam-thtail)/sigth

else

vpoint $=0$

endif

if ((vpoint.1t.8.). and. (chisq(i).1t.20.). and. (u(i).gt.3.3). and. (u(i).1t.11.3). and.

(v(i).gt. -4.0). and. (v(i).1t.4.0)) then

C refill wires.inc with kept track segments

ntrack=ntrack +1

dstnumb (ntrack) $=$ dstnumb (i)

if (i.le.nch1) nch2=nch2+1 !number of heads which point nnwire (ntrack) =nnwire $(i)$

do $j=1, n n w i r e(i)$

$\operatorname{ires}($ ntrack,$j)=$ $\operatorname{ires}(i, j)$

enddo 


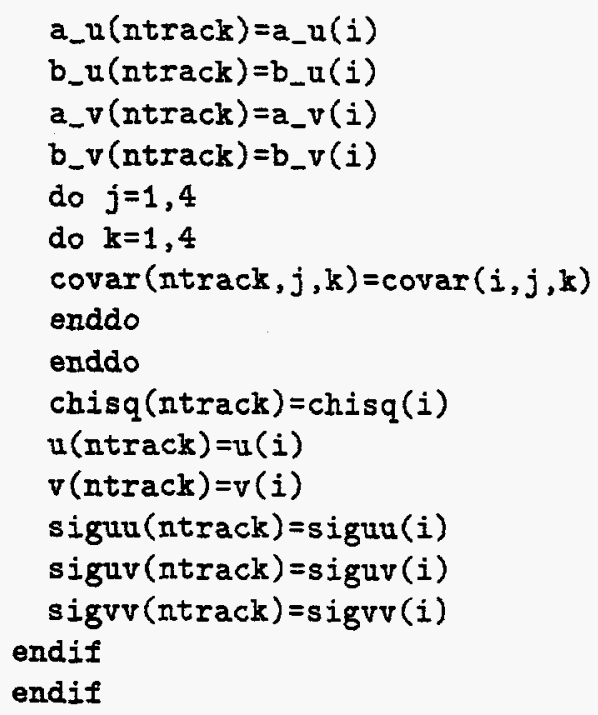

c get $i, j$ for tracks to match nvrtcs $=0$

$C$ increase uncer in pos of tails to account for multiple scattering do $i=1$, ntrack

C first match heads to other segments (smaller uncertainty) do $i=1$, ntrack 


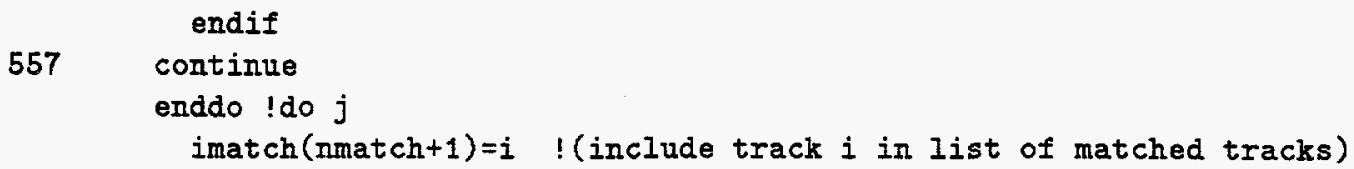




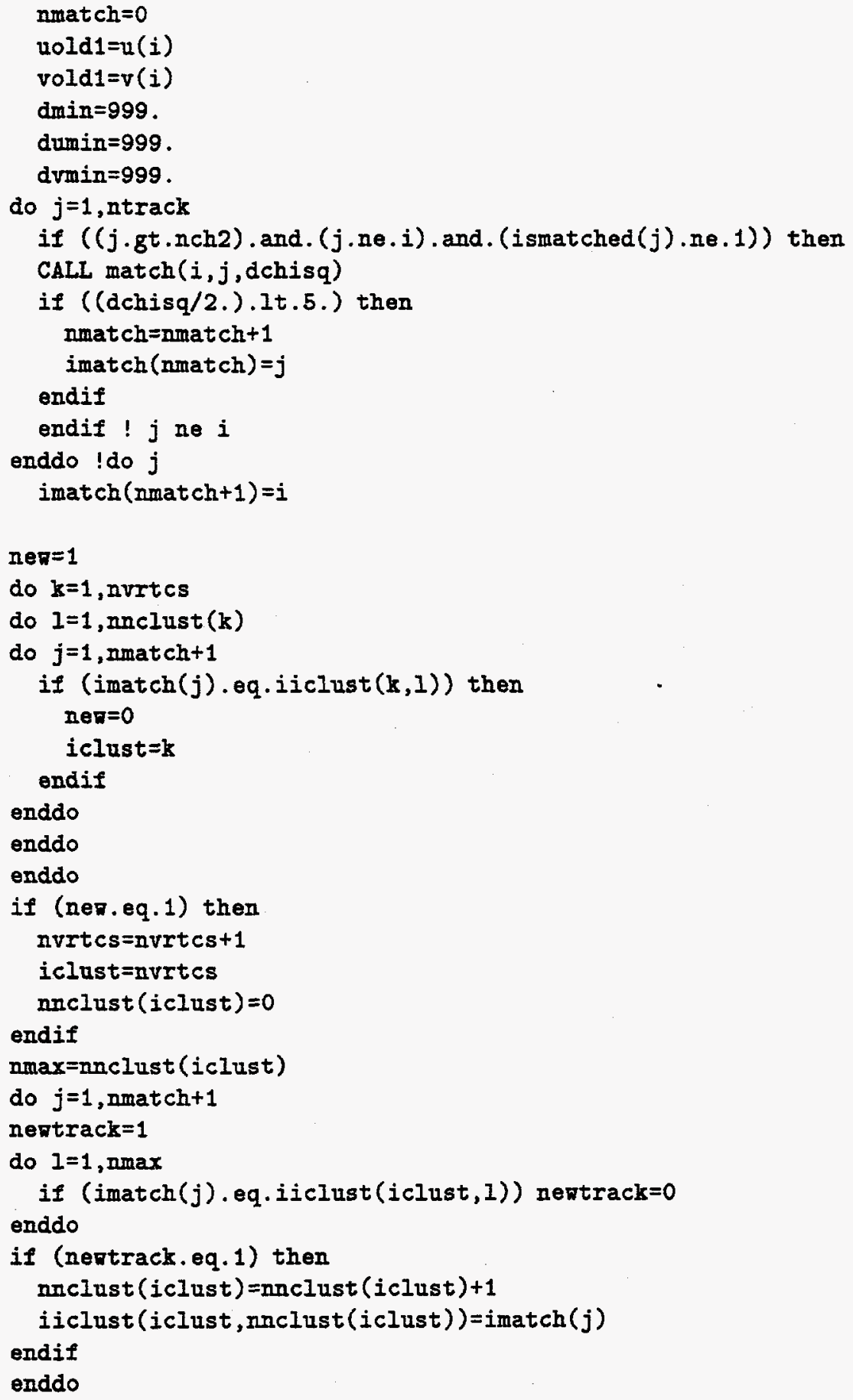




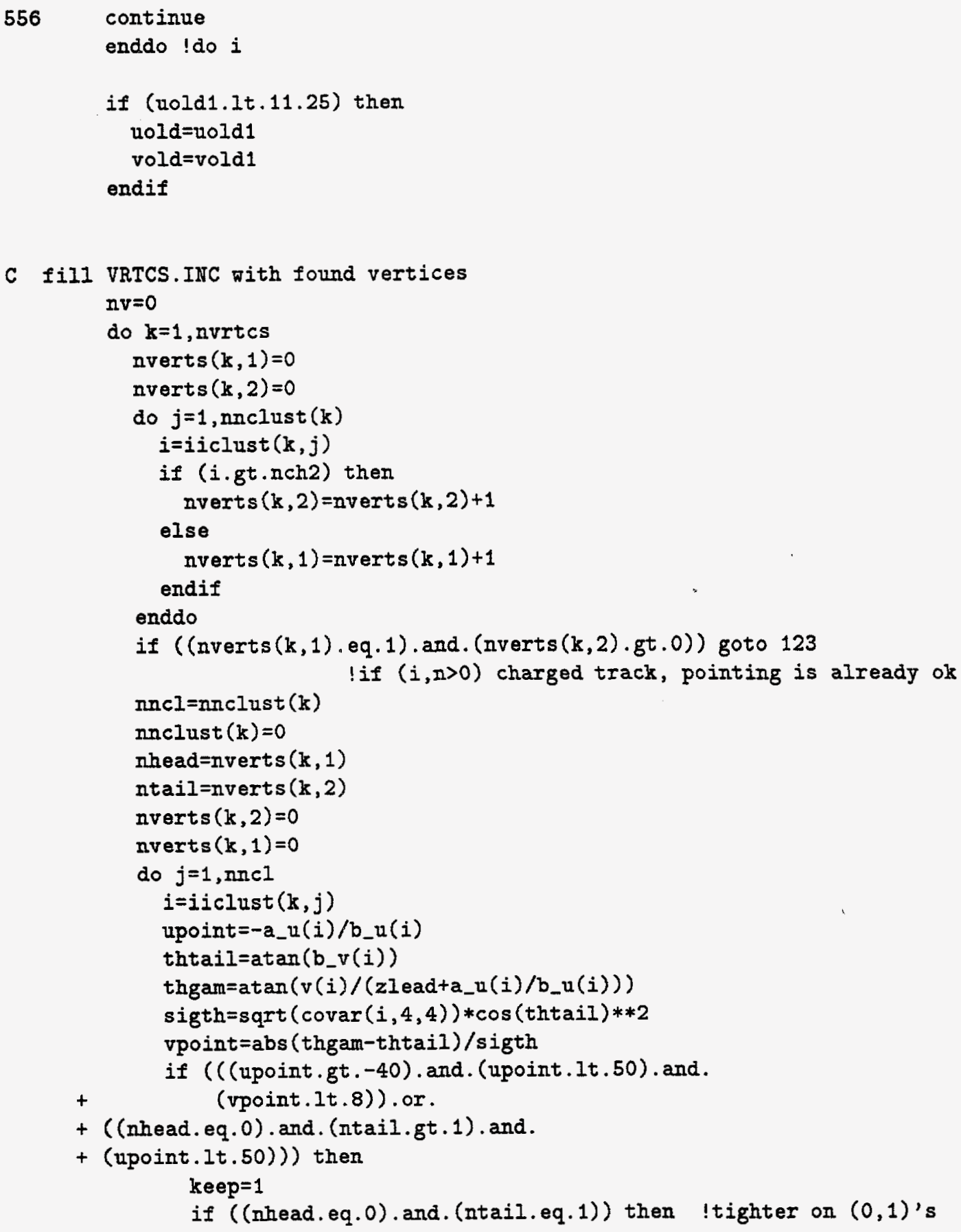




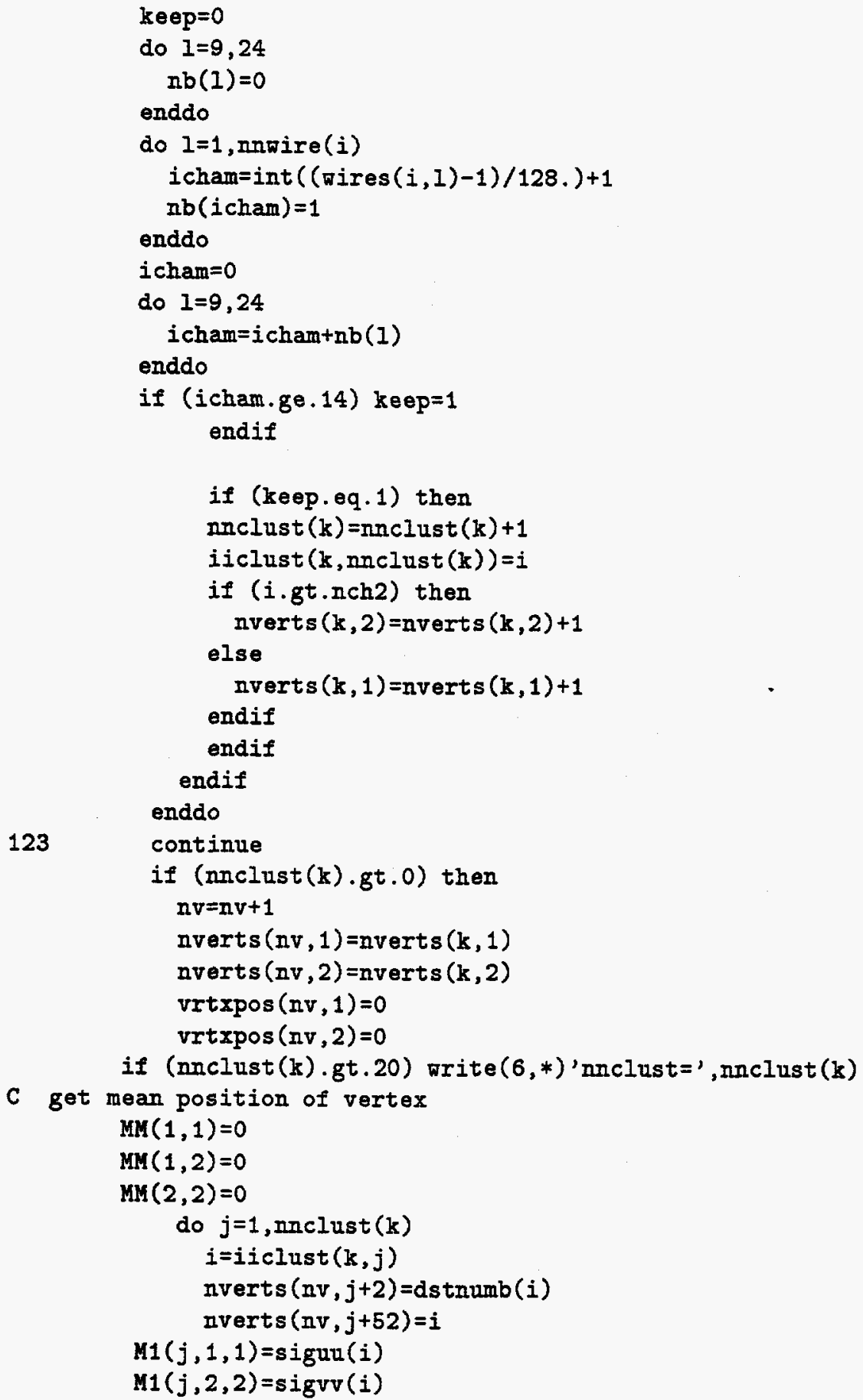




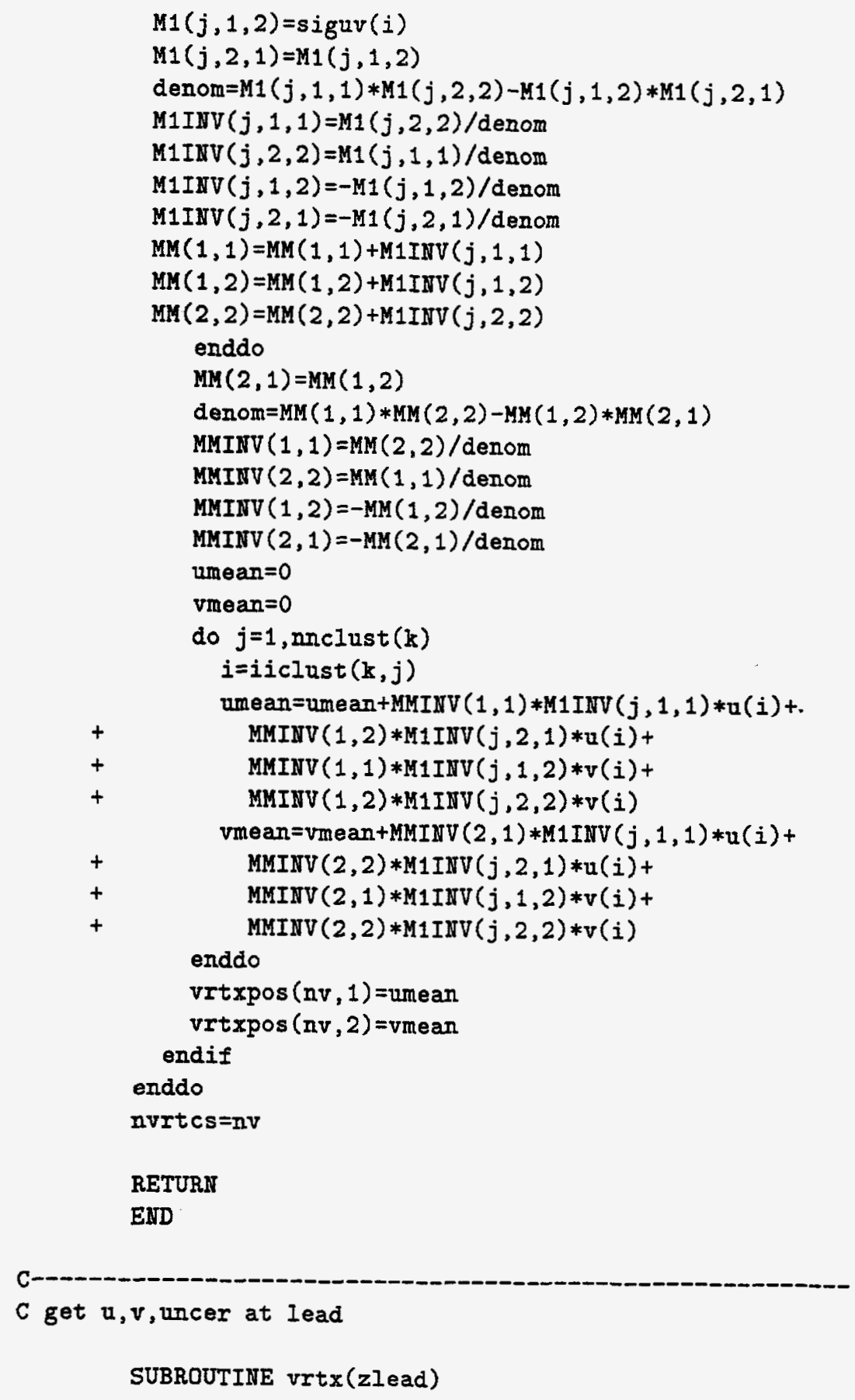




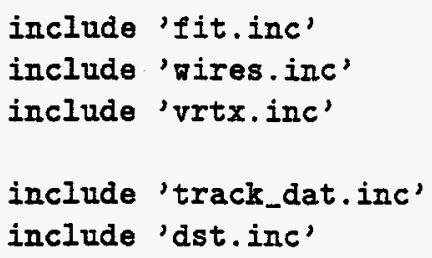

C get chisq of match between pair of tracks

SUBROUTINE match $(i, j$, dchisq)

IMPLICIT NONE

include 'fit.inc'

include 'vrtx.inc'

integer $i, j$

real $C 1(2,2), C 2(2,2), \operatorname{CIINV}(2,2), \operatorname{C2IHV}(2,2), C(2,2), \operatorname{CINV}(2,2)$

real dchisq, umean, vmean, denom

integer nch1, nch2

common/newnch/nch1, nch2

real dist

real dumin, dvmin, dmin, du, dv, nold, vold 


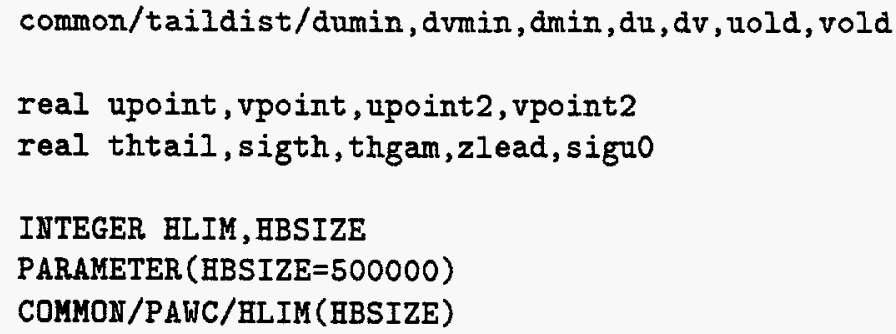


$+\quad \operatorname{CINV}(2,2) *(\operatorname{CIINV}(2,2) * v(i)+\operatorname{C2INV}(2,2) * v(j))$

dchisq $=(u(i)$-umean $) * C 1 \operatorname{INV}(1,1) *(u(i)$-umean $)$

$\begin{array}{ll}+ & +2 *(u(i)-\text { umean }) * \operatorname{C1INV}(1,2) *(v(i)-v m e a n) \\ + & +(v(i)-v m e a n) * \operatorname{C1INV}(2,2) *(v(i)-\text { vmean })\end{array}$

$+\quad+(u(j)$-umean $) * \operatorname{C2INV}(1,1) *(u(j)$-umean $)$

$+\quad+2 *(u(j)-$ umean $) * \operatorname{C} 2 \operatorname{IVV}(1,2) *(v(j)-v m e a n)$

$+\quad+(v(j)-v m e a n) * \operatorname{C2} \operatorname{INV}(2,2) *(v(j)-v m e a n)$

RETURN

END

C

C vrtx.inc

real $u(200), v(200)$, siguu(200), sigvv(200), siguv (200)

common/lead/u, v, siguu, sigvv, siguv

C (determined by subroutine vrtx in vertexer.f)

C position and uncertainty of track at $z=z l e a d$

C

C

VRTCS. INC

integer nvrtcs

integer nverts $(100,102)$

real vrtxpos $(100,2)$

integer ischarged (100), isgamma (100)

common/fndvrts/nvrtcs, nverts, vrtxpos, ischarged, isgama

C (determined by vertexer.f)

C nvrtcs=number of vertices at zlead

C nverts $(i, 1)=$ number of heads in vertex $i$

C nverts $(i, 2)=$ number of tails in vertex $i$

C nverts $(i, 2+j)=d s t-t r a c k$-number of track segment $j$ in vertex $i$

C nverts $(i, 52+j)=f i t t e r-t r a c k-n u m b e r$ of track segment $j$ in vertex $i$

C (determined by chgam.f)

C ischarged( $i) /$ isgamma( $i)=1$ if vertex is a charged track/ gamma

C

$=0$ otherwise 


\section{C.2 Code for classifying vertices as charged tracks or photon conversions: chgam.f}

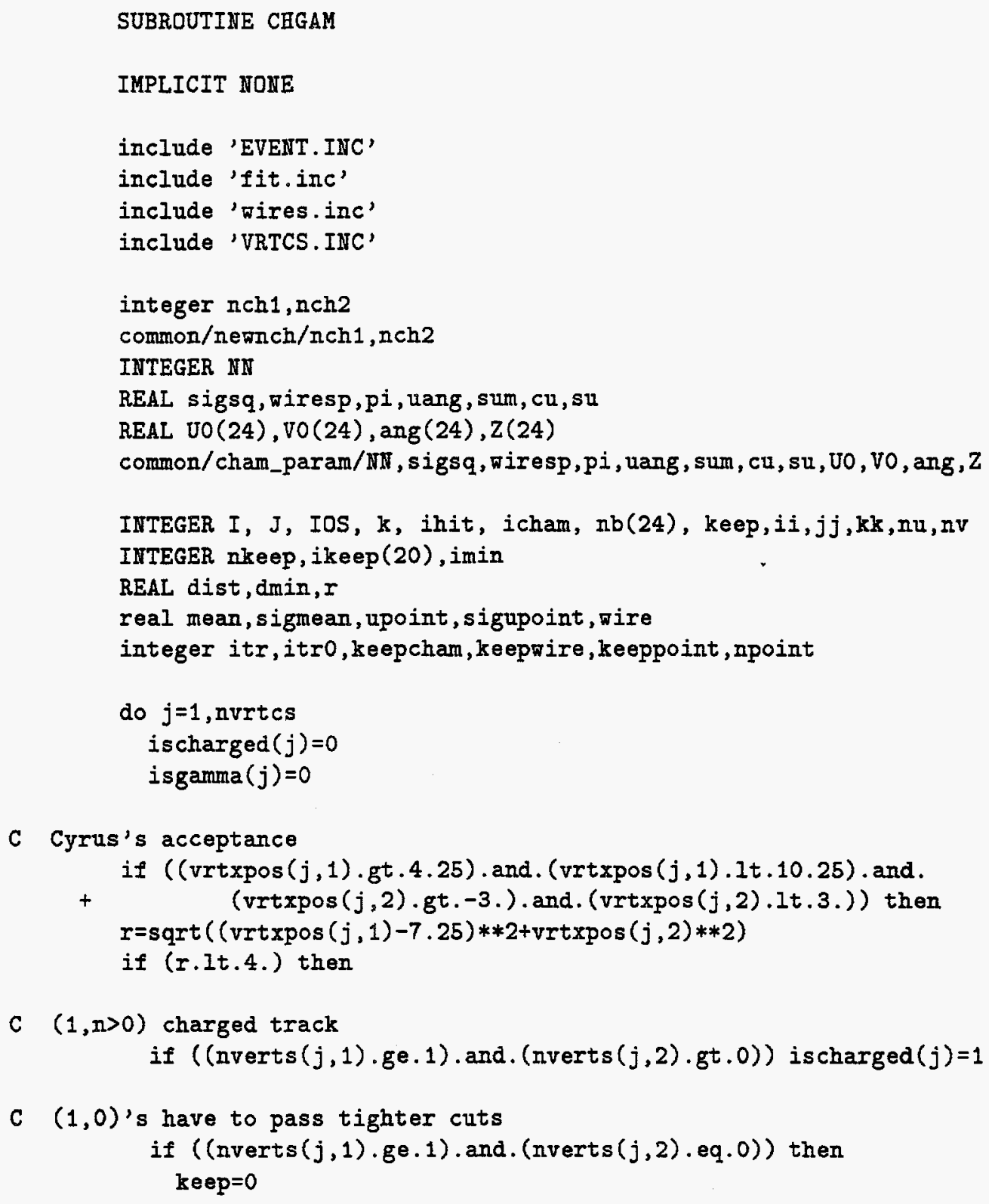




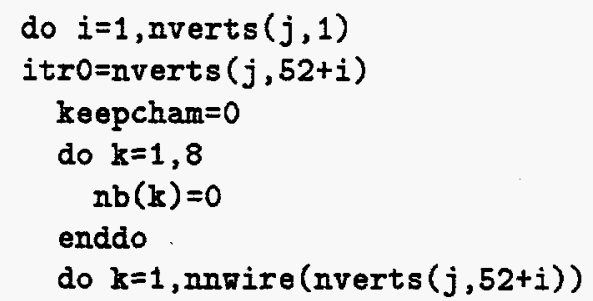


$\mathrm{nv}=\mathrm{nv}+1$

if ((icham.eq. 2).or. (icham.eq.5).or. (icham.eq.8))

$+$ nu=nu+1

endif

enddo

endio

if ((nv.eq.3) or. (nu.eq.3)) keepwire=0

endif

enddo ! ii

endif

enddo $! j j$

C point to mean $z$ of other charged tracks

if (i.eq.1) then

mean $=0$

sigmean $=0$

npoint $=0$

do $j j=1$, nvrtes

if ((nverts $(j j, 1) \cdot g e \cdot 1)$. and. (nverts $(j j, 2) \cdot$ ge.1)) then

do $i i=1, n$ verts $(j j, 1)+n$ verts $(j j, 2)$

if (nverts $(j j, 52+i i)$.le.nch2) then

npoint=npoint +1

itr=nverts $(j j, 52+i i)$

upoint=-a_u(itr)/b_u(itr)

sigupoint $=\left(\operatorname{covar}(i t r, 1,1)+\left(a_{-} u(i t r) / b_{-} u(i t r)\right) * * 2 *\right.$

$\left.+\operatorname{covar}(i t r, 2,2)-2 *\left(a_{-} u(i t r) / b_{-} u(i t r)\right) * \operatorname{covar}(i t r, 1,2)\right)$

$+\quad / b_{-} u($ itr $) * * 2$

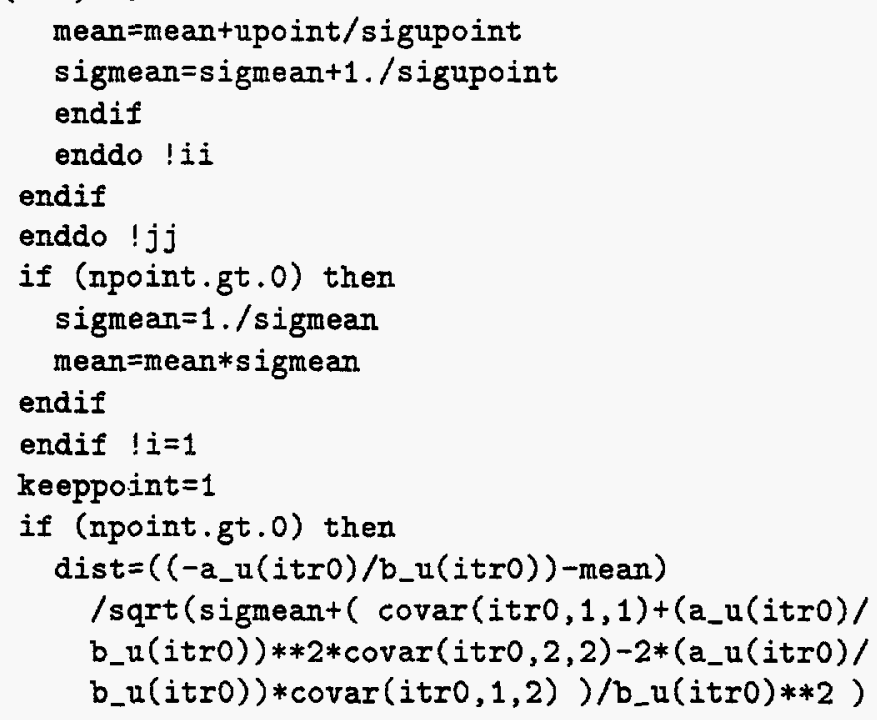




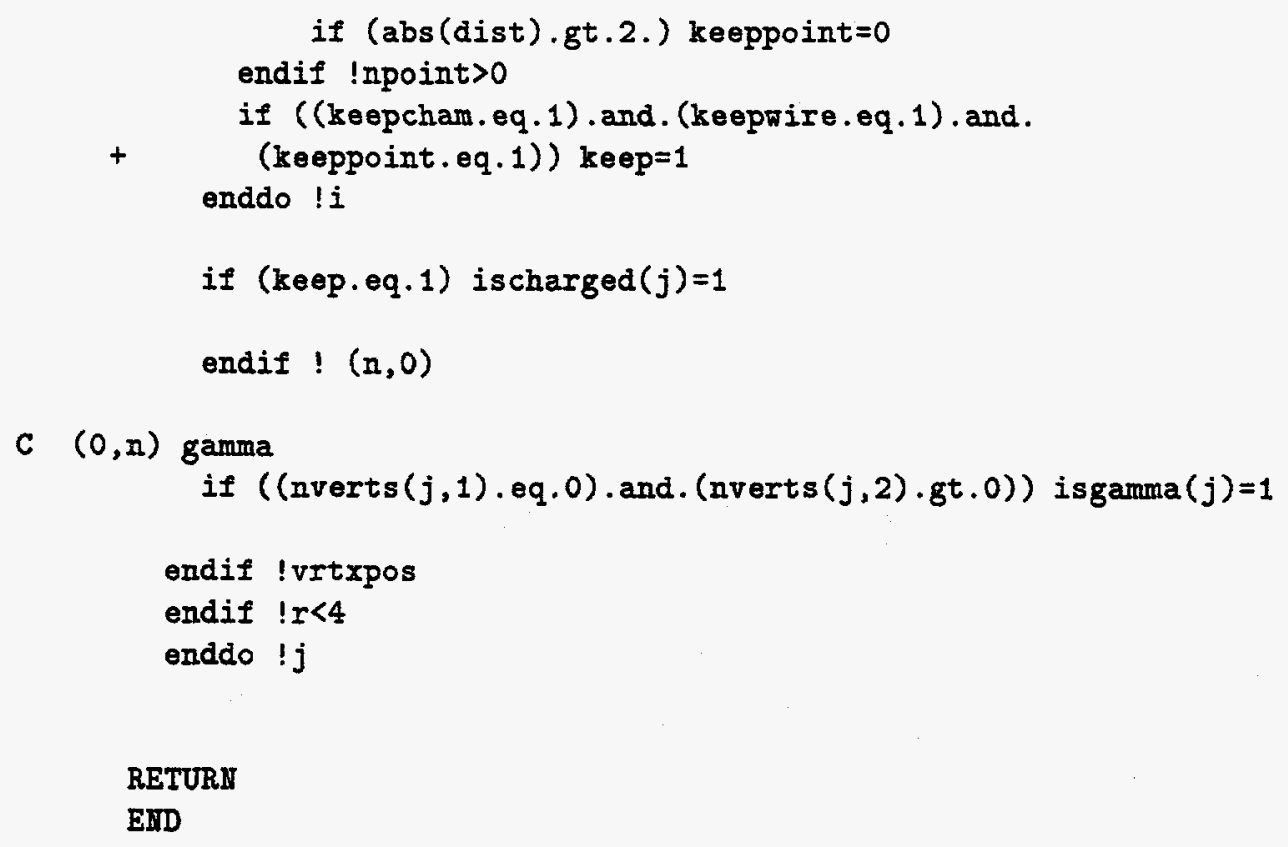




\section{Bibliography}

[1] R. D. Field, Applications of Perturbative QCD, Redwood City, USA: Addison-Wesley (1989).

[2] J. Goldstone, Nuovo Cimiento 19, 154 (1961).

[3] M. Gell-Mann and M. Levy, Nuovo Cimiento, 16, 705 (1960); B. W. Lee, "Chiral Dynamics", ed Gordon and Breach, 1972.

[4] J. I. Kapusta and A. M. Srivastava, Phys. Rev. D 50, 5379 (1994).

[5] A. A. Anselm, Phy. Lett. B 217, 169 (1989).

[6] A. A. Anselm and M. G. Ryskin, Phy. Lett. B 266, 482 (1991).

[7] J. D. Bjorken, Int. J. Mod. Phys. A 7, 4189 (1992).

[8] J. D. Bjorken, Acta Phys. Pol. B 23, 561 (1992).

[9] J.-P. Blaizot and A. Krzywicki, Phys. Rev. D 46, 246 (1992).

[10] K. L. Kowalski and C. C. Taylor, Report No. CWRUTH-92-6, hep$\mathrm{ph} / 9211282$ (unpublished).

[11] K. Rajagopal and F. Wilczek, Nucl. Phys. B399, 395 (1993).

[12] J. D. Bjorken, K. L. Kowalski, and C. C. Taylor, in Proceedings of Les Recontres de la Vallée D' Aoste, La Thuile, Italy, 1993, edited by M. Greco (Editions Frontieres, Gif-sur-Yvette, France, 1993), p. 507.

[13] M. Martinis, V. Mikata-Martinis, A. Švarc, and J. Črnugelj, Phys. Rev. D 51, 2482 (1995); Fizika B3, 197 (1994); M. Martinis, V. MikataMartinis, and J. Crnugelj, Phys. Rev. C 521073 (1995).

[14] R. D. Amado et al., Phys. Rev. Lett. 72, 970 (1994).

[15] B. Müller, Rep. Prog. Phys. 58, 611 (1995). 
[16] A good review can be found in K. Rajagopal, in Quark-Gluon Plasma 2, edited by R. Hwa, World Scientific, Singapore, (1995), p. 454.

[17] I. V. Andreev, JETP Lett. 33, 67 (1981).

[18] V. Karmanov and A. Kudrjavtsev, Report No. ITEP-88, 1983, (unpublished).

[19] D. Horn and R. Silver, Ann. Phys. (N.Y.) 66, 509 (1971).

[20] L. T. Beradzei et al. (Chacaltaya-Pamir Collaboration), Nucl. Phys. B370, 365 (1992); C. M. G. Lattes, Y. Fujimoto and S. Hasegawa, Phys. Rep. 65, 151 (1980). Chacaltaya-Pamir Collaboration, ICRReport-258-91-27 (unpublished) and references cited therein.

[21] S. Hasegawa, (Chacaltaya-Pamir Collaboration), ICR-Report-151-87-5 (unpublished).

[22] See, for example, K. Goulianos, "A Comment on Centauro Production", Comm. on Nucl. and Par. Phys. 17, 195 (1987); K. Goulianos, "Exotic Diffraction Dissociation in Cosmic Rays and Hadron Colliders", Proceedings of VIIth Int. Symp. on Very High Energy Cosmic Ray Interactions, edited by L. Jones, Ann Arbor, June 1992.

[23] K. Goulianos, Phys. Rep. 101, 169 (1983).

[24] J. J. Lord and J. Iwai, Intl. Conference on High Energy Physics, Dallas, TX, August 1992; J. Iwai, Report No. UWSEA 92-06; Y. Takahashi (JACEE Collaboration), Proceedings of VIIth Int. Symp. on Very High Energy Cosmic Ray Interactions, edited by L. Jones, Ann Arbor, June 1992; H. Wilczynski (JACEE Collaboration), talk given at the IXth International Symposium on Very High Energy Cosmic Ray Interactions, Karlsruhe, Germany, August 1996.

[25] P. L. Melese (CDF Collaboration), FERMILAB-CONF-96-205-E, July 1966.

[26] K. Alpgård et al., Phys. Lett. B 115, 71 (1982); G. J. Alner et al., Phys. Lett. B 180, 415 (1986).

[27] G. J. Alner et al., Phys. Rep. 154, 247 (1987).

[28] G. Arnison et al., Phys. Lett. B 122, 189 (1983).

[29] F. Abe et al., Phys. Rev. D 41, 2330 (1990). 
[30] G. Arnison et al., Phys. Lett. B 123, 108 (1983).

[31] C. J. Liapis, $\mathrm{PhD}$ thesis, Yale University, New Haven, CT, 1995; R. Harr, et al, hep-ex/9703002, submitted to Phys. Lett. B.

[32] R. E. Ansorge et al., Z. Phys. C 43, 75 (1989).

[33] G. J. Alner et al., Nucl. Phys. B291, 445 (1987).

[34] P. R. Bevington, B. D. Anderson, R. J. Barrett, and F. H. Cverna, Nucl. Inst. Meth. 129, 373 (1975).

[35] Based on private communication from HERA-B to Bjorken.

[36] R. M. Barnett et al., Phys. Rev. D 54, 1 (1996).

[37] F. Abe et. al, Phys. Rev. D, 50, 5550 (1994); F. Abe et. al, Phys. Rev. D, 50, 5518 (1994); F. Abe et. al, Phys. Rev. D, 50, 5535 (1994).

[38] The calorimeter cells made by the University of Michigan are based on a design described in H. Fessier et al., Nucl. Instr. Meth. A 240, 284 (1985).

[39] K. Barish et al., to be submitted to NIM; C. Pruneau (E864 Collaboration), Proceedings of the VI International Conference on Calorimetry in High Energy Physics, Fracati, Italy, July 1996 (in print); C. Pruneau (E864 Collaboration), Proceedings of the 12th Winter Workshop on Nuclear Dynamics, Snowbird, Utah, Feb 3-10, 1996, published in Advance in Nuclear Dynamics 2, edited by W.Bauer and G.D.Westfall, Plenum, p. 199.

[40] J. D. Bjorken, "A Full Acceptance Detector for SSC Physics at Low and Intermediate Mass Scales", SSC EoI-19, SLAC-PUB-5545 (May 1991).

[41] Fermilab Proposal P-864 (J. Bjorken and M. Longo, spokespersons), "MAX", unpublished.

[42] T. Sjostrand and M. Bengtsson, Computer Physics Commun. 43, 367 (1987); H.-U. Bengtsson and T. Sjostrand, Computer Physics Commun. 46, 43 (1987).

[43] T. Sjostrand, CERN-TH.7112/93 (1993).

[44] Parton-model phenomenology is described in M. E. Peskin and D. V. Schroeder, An Introduction to Quantum Field Theory, Reading, USA: Addison-Wesley (1995). 
[45] B. Andersson, G. Gustafson, G. Ingelman and T. Sjostrand, Phys. Rep. 97, 31 (1983).

[46] GEANT - Detector Description and Simulation Tool, CERN, PM0062 (1993).

[47] J. Pumplin, Phys. Rev. D 50, 6811 (1994).

[48] The Forward Muon Spectrometer of ALICE - Addendum to the Technical Proposal for A Large Ion Collider at the CERN LHC, CERN/LHCC 96-32, LHCC/P3-Addendum 1, 1996.

[49] The combinatorial tracker was written by K. DelSignore. Another tracker used a hougher algorithm and is documented in the senior thesis of E. Kangas, CWRU, 1995. A third tracker written by T. Jenkins was based on a track-following algorithm.

[50] P. R. Bevington, Data Reduction and Error Analysis for the Physical Sciences, New York, McGraw-Hill (1969).

[51] P. Carruthers and C. C. Shih, Int. J. Mod. Phys. A 2, 1447 (1987).

[52] C. Geich-Gimbel, Int. J. Mod. Phys. A 4, 1527 (1989).

[53] I. M. Dremin, Mod. Phys. Lett. A 8, 2747 (1993); Pis'ma Zh. Eksp. Teor. Fiz. 59, 561 (1994) [JETP Lett. 59, 585 (1994)].

[54] I. M. Dremin and R. C. Hwa, Phys. Rev. D 49, 5805 (1994).

[55] For a review, see E. A. DeWolf, I. M. Dremin and W. Kittel, Phys. Rep. 270, 1 (1996).

[56] G. H. Thomas and B. R. Webber, Phys. Rev. D 9, 3113 (1974).

[57] L. Diósi, Nucl. Instrum. Methods 138, 241 (1976).

[58] L. Diósi, Nucl. Instrum. Methods 140, 533 (1977).

[59] L. Diósi and B. Lukács, Phys. Lett. B 206, 707 (1988).

[60] T. C. Brooks et al., accepted for publication in Phys. Rev. D, tenatively to appear in May 1997.

[61] I. S. Gradshteyn and I. M. Ryzhik, Table of Integrals, Series, and Products, Academic Press, Orlando, 1980, p. 20.

[62] M. Spyropoulou-Stassinaki et al., Nucl. Phys. A525, 487c, (1991). 